\title{
Modelos INAR e RCINAR, estimação e aplicação
}

Tiago de Almeida Cerqueira Lima

\author{
DisSERTAÇÃO APRESENTADA \\ $\mathrm{AO}$ \\ Instituto De Matemática e EstatísticA \\ DA \\ Universidade DE SÃo PAUlo \\ PARA \\ OBTENÇÃO DO TÍTULO \\ $\mathrm{DE}$ \\ Mestre em CiÊnCIAS \\ Programa: Estatística \\ Orientador: Prof ${ }^{\mathrm{a}}$. Dr ${ }^{\mathrm{a}}$. Clélia Maria Castro Toloi
}

São Paulo, fevereiro de 2013 


\section{Modelos INAR e RCINAR, estimação e aplicação}

Esta versão da dissertação contém as correções e alterações sugeridas pela Comissão Julgadora durante a defesa da versão original do trabalho, realizada em 07/05/2013. Uma cópia da versão original está disponível no

Instituto de Matemática e Estatística da Universidade de São Paulo.

Comissão Julgadora:

- Prof ${ }^{\mathrm{a}}$. Dr ${ }^{\mathrm{a}}$. Clélia Maria Castro Toloi (orientadora) - IME-USP

- Prof $^{\mathrm{a}}$. Dr ${ }^{\mathrm{a}}$. Chang Chiann - IME-USP

- Prof ${ }^{a}$. Dra ${ }^{a}$. Sílvia Regina Costa Lopes - IM - UFRGS 


\section{Agradecimentos}

Eu agradeço primeiramente aos meus pais, Clarisse e Raimundo que sempre me incentivaram a estudar, desde a época de trabalho duro na oficina mecânica, me apoiando na universidade até os dias de hoje. Minha amada mãe que mesmo nesses últimos tempos difíceis pra ela, me deu força.

Agradeço à minha orientadora, Prof ${ }^{\mathrm{a}}$. Clélia Maria Castro Toloi, que além da orientação nesse trabalho me apoiou também em questões pessoais.

À minha namorada, Ana Carolina, que passou tantos momentos difíceis ao meu lado e nunca se deixou abater e sempre me contagiou com sua alegria.

Por fim, quero agradecer ao meu grande amigo Danilo Mustafa que é como um irmão, aos meus amigos de colégio e faculdade, à todos da Serasa que de alguma forma tem uma parte contribuinte nesse trabalho, em especial à minha amiga Karina Angélica e Guilherme Fernandes que colaboraram desde o início da minha pós-graduação. 


\section{Resumo}

Neste trabalho primeiramente apresentamos um modelo para uma sequência estacionária de valores inteiros (processo de contagem) autoregressivo de ordem p (INAR(p)). Depois disso, mostraremos uma extensão desse processo, chamado modelo autoregressivo inteiro com coeficientes aleatórios $(\operatorname{RCINAR}(\mathrm{p}))$.

Para ambos os modelos, apresentamos suas propriedades assim como diferentes métodos de estimação de seus parâmetros.

Os resultados da simulação e comparação dos estimadores são mostrados. Finalmente os modelos são aplicados em dois conjuntos de dados reais: Número mensal de empresas em falência; Número mensal de consultas no bureau de crédito.

Palavras-chave: Séries temporais inteiras, Modelo INAR, Modelo RCINAR, métodos de estimação. 


\section{Abstract}

At this work we first present a model for stationary sequence of integer-valued random variables (counting process) referred to as the integer-valued autoregressive of order p (INAR(p)) process. After this we show an extension of this process, called random coefficient integer-valued autoregressive process (RCINAR(p)).

For both models we present its properties as well as different methods of estimation of its parameters.

Simulation results and the comparison of the estimators are reported. Finally the models are applied to two real data sets: monthly number of companies with bankruptcy; monthly number of enquiries in credit bureau.

Keywords: Integer time series, INAR model, RCINAR model, estimation methods. 


\section{Sumário}

$\begin{array}{ll}\text { Lista de Abreviaturas } & \text { ix }\end{array}$

Lista de Figuras $\quad$ xi

Lista de Tabelas $\quad$ xv

1 Introdução $\quad 1$

1.1 Considerações Preliminares . . . . . . . . . . . . . . . . . . . . . 1

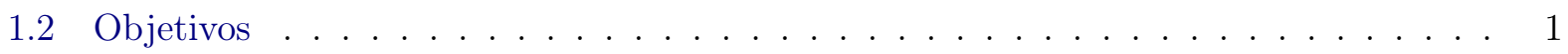

1.3 Contribuições . . . . . . . . . . . . . . . . . . . . . . 2

1.4 Organização do Trabalho . . . . . . . . . . . . . . . . . . . . 2

2 Conceitos $\quad 3$

2.1 Séries Temporais . . . . . . . . . . . . . . . . . . . . . . 3

2.2 Estacionariedade . . . . . . . . . . . . . . . . . . . . 3

2.2 .1 Função de autocorrelação . . . . . . . . . . . . . . . . . . . . . 4

2.2.2 Função de autocorrelação parcial . . . . . . . . . . . . . . . . . . . . 4

2.3 Modelo Autorregressivo . . . . . . . . . . . . . . . . . . . . . 5

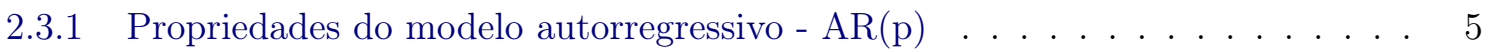

2.4 Medida de acurácia . . . . . . . . . . . . . . . . . . . . . . . 7

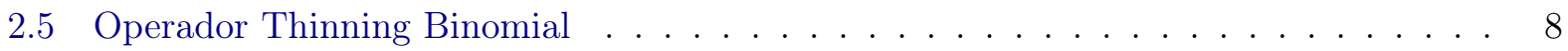

3 Modelos autorregressivos inteiros $\quad 9$

3.1 Modelos INAR . . . . . . . . . . . . . . . . . . . . . . . 10

3.1 .1 Modelo INAR $(1) \ldots \ldots \ldots \ldots \ldots \ldots \ldots \ldots$

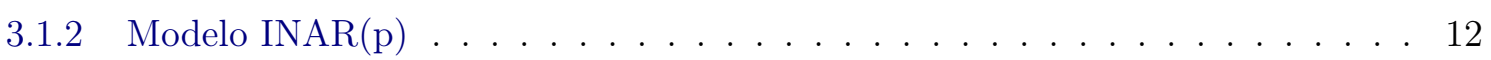

3.1 .3 Métodos de estimação . . . . . . . . . . . . . . . . . . . . . . . 13

3.1 .4 Estudo de simulação . . . . . . . . . . . . . . . . . . . . . . . 17

3.2 Modelos RCINAR . . . . . . . . . . . . . . . . . . . . . . 39

3.2 .1 Modelo RCINAR $(\mathrm{p}) \ldots \ldots \ldots \ldots \ldots$

3.2 .2 Métodos de estimação . . . . . . . . . . . . . . . . . . . . . . . . . . . 40

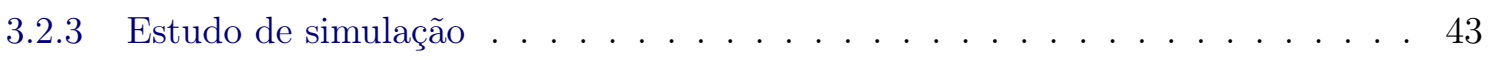

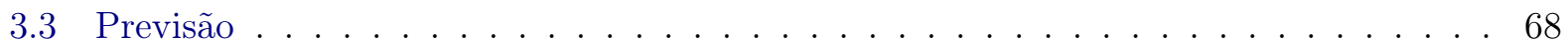


viii SUMÁRIO

4 Aplicação $\quad 69$

4.1 Dados de falência . . . . . . . . . . . . . . . . . . . . . . . 69

4.2 Dados de crédito . . . . . . . . . . . . . . . . . . . . . 73

5 Conclusões $\quad 77$

5.1 Sugestões para Pesquisas Futuras . . . . . . . . . . . . . . . . 77

$\begin{array}{ll}\text { A Código implementado } & 79\end{array}$

$\begin{array}{ll}\text { Referências Bibliográficas } & 89\end{array}$ 


\title{
Lista de Abreviaturas
}

\author{
ACF Função de autocorrelação (Autocorrelation Function) \\ PACF Função de autocorrelação parcial (Partial Autocorrelation Function) \\ EQM Erro quadrático médio \\ INAR Processo inteiro autorregressivo (Integer Autorregressive Process) \\ RCINAR Processo inteiro autorregressivo com coeficiente aleatório \\ (Random Coefficient Integer Autorregressive Process) \\ MV Máxima verossimilhança \\ MVC Máxima verossimilhança condicional \\ MQC Mínimos quadrados condicionais \\ QMV Quase máxima verossimilhança \\ AR Autorregressivo \\ MA Média móvel
}




\section{Lista de Figuras}

2.1 Série simulada - AR(1) com $\phi_{1}=0,74 \ldots \ldots \ldots \ldots \ldots \ldots$

2.2 Autocorrelação e Autocorrelação parcial amostral de um modelo AR(1) gerado com

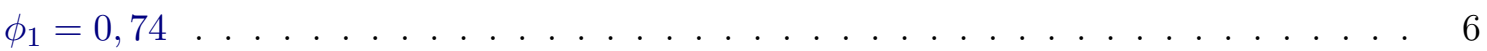

2.3 Autocorrelação e Autocorrelação parcial teóricas do modelo $\operatorname{AR}(1) \operatorname{com} \phi_{1}=0,74$. 7

3.1 Autocorrelação e Autocorrelação parcial teórica: $\operatorname{INAR(1)~} \operatorname{com} \alpha=0,35$ e $\lambda=20 . \quad$. 11

3.2 Autocorrelação e Autocorrelação parcial teórica: $\operatorname{INAR}(2) \operatorname{com}\left(\alpha_{1}, \alpha_{2}\right)=(0,3,0,18)$

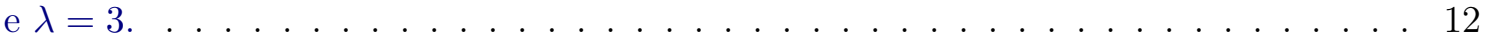

3.3 Autocorrelação e Autocorrelação parcial teórica: $\operatorname{INAR}(5) \operatorname{com}\left(\alpha_{1}, \alpha_{2}, \alpha_{3}, \alpha_{4}, \alpha_{5}\right)=$ $(0,22,0,15,0,2,0,08,0,1)$ e $\lambda=1 \ldots \ldots \ldots \ldots \ldots$

3.4 Gráfico de uma série simulada, $\operatorname{INAR}(1): \alpha=0,3$ e $\lambda=1 \ldots \ldots \ldots$

3.5 Histograma e boxplot da série simulada, $\operatorname{INAR}(1): \alpha=0,3$ e $\lambda=1 \ldots \ldots \ldots$

3.6 Autocorrelação e Autocorrelação parcial amostrais $\operatorname{INAR}(1): \alpha=0,3$ e $\lambda=1 \ldots$. . 18

3.7 Boxplot das estimativas de $\alpha$ para o modelo gerado por $X_{t}=0,3 \circ X_{t-1}+I_{t}$. . . . 19

3.8 Boxplot das estimativas de $\lambda$ para o modelo gerado por $X_{t}=0,3 \circ X_{t-1}+I_{t}$. . . . 19

3.9 Histograma alisado do viés de $\hat{\alpha}$ para o modelo gerado por $X_{t}=0,3 \circ X_{t-1}+I_{t}$. . . 20

3.10 Histograma alisado do viés de $\hat{\lambda}$ para o modelo gerado por $X_{t}=0,3 \circ X_{t-1}+I_{t}$. . . 20

3.11 Gráfico de uma série simulada, $\operatorname{INAR}(1): \alpha=0,5$ e $\lambda=1 \ldots \ldots \ldots \ldots$

3.12 Histograma e boxplot da série simulada, $\operatorname{INAR}(1): \alpha=0,5$ e $\lambda=1 \ldots \ldots \ldots$

3.13 Autocorrelação e Autocorrelação parcial amostrais $\operatorname{INAR}(1): \alpha=0,5$ e $\lambda=1$. . . . 21

3.14 Boxplot das estimativas de $\alpha$ para o modelo gerado por $X_{t}=0,5 \circ X_{t-1}+I_{t}$. . . . 22

3.15 Boxplot das estimativas de $\lambda$ para o modelo gerado por $X_{t}=0,5 \circ X_{t-1}+I_{t}$. . . . 22

3.16 Histograma alisado do viés de $\hat{\alpha}$ para o modelo gerado por $X_{t}=0,5 \circ X_{t-1}+I_{t}$. . . 23

3.17 Histograma alisado do viés de $\hat{\lambda}$ para o modelo gerado por $X_{t}=0,5 \circ X_{t-1}+I_{t}$. . . 23

3.18 Gráfico de uma série simulada, $\operatorname{INAR}(1): \alpha=0,7$ e $\lambda=1 \ldots \ldots \ldots . \ldots . \ldots 24$

3.19 Histograma e boxplot da série simulada, $\operatorname{INAR}(1): \alpha=0,7$ e $\lambda=1 \ldots$. . . . . . . 24

3.20 Autocorrelação e Autocorrelação parcial amostrais $\operatorname{INAR}(1): \alpha=0,7$ e $\lambda=1$. . . 24

3.21 Boxplot das estimativas de $\alpha$ para o modelo gerado por $X_{t}=0,7 \circ X_{t-1}+I_{t}$. . . . 25

3.22 Boxplot das estimativas de $\lambda$ para o modelo gerado por $X_{t}=0,7 \circ X_{t-1}+I_{t}$. . . . 25

3.23 Histograma alisado do viés de $\hat{\alpha}$ para o modelo gerado por $X_{t}=0,7 \circ X_{t-1}+I_{t}$. . . 26

3.24 Histograma alisado do viés de $\hat{\lambda}$ para o modelo gerado por $X_{t}=0,7 \circ X_{t-1}+I_{t}$. . . 26

3.25 Gráfico de uma série simulada, $\operatorname{INAR}(2): \alpha_{1}=0,3, \alpha_{2}=0,3$ e $\lambda=1 \ldots \ldots$. . . . 29

3.26 Histograma e boxplot da série simulada, $\operatorname{INAR}(2): \alpha_{1}=0,3, \alpha_{2}=0,3$ e $\lambda=1$. . . 29 
3.27 Autocorrelação e Autocorrelação parcial amostrais $\operatorname{INAR}(2): \alpha_{1}=0,3, \alpha_{2}=0,3$ e $\lambda=1$.

3.28 Boxplot das estimativas de $\alpha_{1}$ para o modelo gerado por $X_{t}=0,3 \circ X_{t-1}+0,3 \circ X_{t-2}+I_{t} .30$

3.29 Boxplot das estimativas de $\alpha_{2}$ para o modelo gerado por $X_{t}=0,3 \circ X_{t-1}+0,3 \circ X_{t-2}+I_{t} .30$

3.30 Boxplot das estimativas de $\lambda$ para o modelo gerado por $X_{t}=0,3 \circ X_{t-1}+0,3 \circ X_{t-2}+I_{t}$. 30

3.31 Histograma alisado do viés de $\hat{\alpha_{1}}$ para o modelo gerado por $X_{t}=0,3 \circ X_{t-1}+0,3 \circ$ $X_{t-2}+I_{t}$.

3.32 Histograma alisado do viés de $\hat{\alpha_{2}}$ para o modelo gerado por $X_{t}=0,3 \circ X_{t-1}+0,3 \circ$ $X_{t-2}+I_{t}$.

3.33 Histograma alisado do viés de $\hat{\lambda}$ para o modelo gerado por $X_{t}=0,3 \circ X_{t-1}+0,3 \circ$

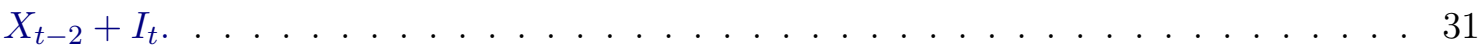

3.34 Gráfico de uma série simulada, $\operatorname{INAR}(2): \alpha_{1}=0,3, \alpha_{2}=0,5$ e $\lambda=1 \ldots \ldots$. . . . 32

3.35 Histograma e boxplot da série simulada, $\operatorname{INAR}(2): \alpha_{1}=0,3, \alpha_{2}=0,5$ e $\lambda=1$. . . 32

3.36 Autocorrelação e Autocorrelação parcial amostrais $\operatorname{INAR}(2): \alpha_{1}=0,3, \alpha_{2}=0,5$ e

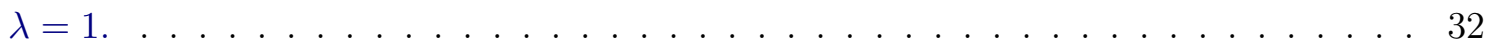

3.37 Boxplot das estimativas de $\alpha_{1}$ para o modelo gerado por $X_{t}=0,3 \circ X_{t-1}+0,5 \circ X_{t-2}+I_{t} .33$

3.38 Boxplot das estimativas de $\alpha_{2}$ para o modelo gerado por $X_{t}=0,3 \circ X_{t-1}+0,5 \circ X_{t-2}+I_{t} .33$

3.39 Boxplot das estimativas de $\lambda$ para o modelo gerado por $X_{t}=0,3 \circ X_{t-1}+0,5 \circ X_{t-2}+I_{t} .33$

3.40 Histograma alisado do viés de $\hat{\alpha_{1}}$ para o modelo gerado por $X_{t}=0,3 \circ X_{t-1}+0,5 \circ$ $X_{t-2}+I_{t} \ldots \ldots \ldots \ldots \ldots \ldots \ldots \ldots \ldots \ldots \ldots \ldots \ldots \ldots \ldots \ldots \ldots$

3.41 Histograma alisado do viés de $\hat{\alpha_{2}}$ para o modelo gerado por $X_{t}=0,3 \circ X_{t-1}+0,5 \circ$

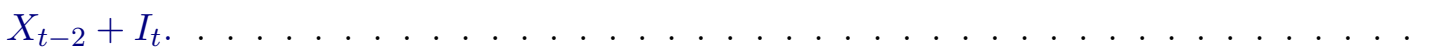

3.42 Histograma alisado do viés de $\hat{\lambda}$ para o modelo gerado por $X_{t}=0,3 \circ X_{t-1}+0,5 \circ$

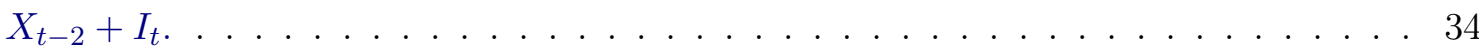

3.43 Gráfico de uma série simulada, $\operatorname{INAR}(2): \alpha_{1}=0,5, \alpha_{2}=0,3$ e $\lambda=1 \ldots \ldots$. . . 35

3.44 Histograma e boxplot da série simulada, $\operatorname{INAR}(2): \alpha_{1}=0,5, \alpha_{2}=0,3$ e $\lambda=1$. . . 35

3.45 Autocorrelação e Autocorrelação parcial amostrais $\operatorname{INAR}(2): \alpha_{1}=0,5, \alpha_{2}=0,3$ e

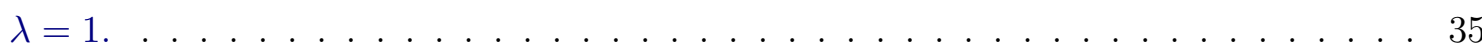

3.46 Boxplot das estimativas de $\alpha_{1}$ para o modelo gerado por $X_{t}=0,5 \circ X_{t-1}+0,3 \circ X_{t-2}+I_{t}$. 36

3.47 Boxplot das estimativas de $\alpha_{2}$ para o modelo gerado por $X_{t}=0,5 \circ X_{t-1}+0,3 \circ X_{t-2}+I_{t}$. 36

3.48 Boxplot das estimativas de $\lambda$ para o modelo gerado por $X_{t}=0,5 \circ X_{t-1}+0,3 \circ X_{t-2}+I_{t} .36$

3.49 Histograma alisado do viés de $\hat{\alpha_{1}}$ para o modelo gerado por $X_{t}=0,5 \circ X_{t-1}+0,3 \circ$

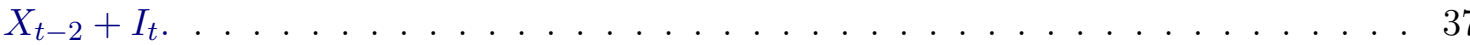

3.50 Histograma alisado do viés de $\hat{\alpha_{2}}$ para o modelo gerado por $X_{t}=0,5 \circ X_{t-1}+0,3 \circ$

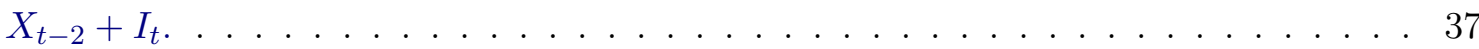

3.51 Histograma alisado do viés de $\hat{\lambda}$ para o modelo gerado por $X_{t}=0,5 \circ X_{t-1}+0,3 \circ$

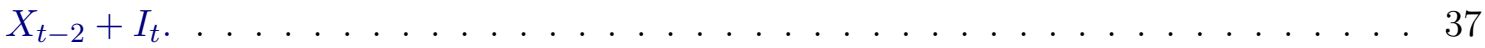

3.52 Distribuição Beta considerada para o estudo. . . . . . . . . . . . . . . . 44

3.53 Histograma e ACF do $\phi_{t}$ simulado, $\operatorname{RCINAR}(1) \ldots \ldots \ldots \ldots \ldots$

3.54 Gráfico de uma série simulada, $\operatorname{RCINAR}(1): E\left[\phi_{t}\right]=0,3$ e $\lambda=1 \ldots \ldots \ldots$

3.55 Histograma e boxplot da série simulada, $\operatorname{RCINAR(1):~} E\left[\phi_{t}\right]=0,3$ e $\lambda=1 \ldots \ldots$. . . 45

3.56 Autocorrelação e autocorrelação parcial amostrais $\operatorname{RCINAR}(1): E\left[\phi_{t}\right]=0,3$ e $\lambda=1 . \quad 46$

3.57 Boxplot das estimativas de $E\left[\phi_{t}\right]$ para o modelo gerado por $X_{t}=\phi_{t} \circ X_{t-1}+I_{t} \ldots .46$ 
3.58 Boxplot das estimativas de $\lambda$ para o modelo gerado por $X_{t}=\phi_{t} \circ X_{t-1}+I_{t} \ldots \ldots$. . 46

3.59 Histograma alisado do viés de $\hat{\Phi}$ para o modelo gerado por $X_{t}=\phi_{t} \circ X_{t-1}+I_{t}$. . . 47

3.60 Histograma alisado do viés de $\hat{\lambda}$ para o modelo gerado por $X_{t}=\phi_{t} \circ X_{t-1}+I_{t}$. . . 47

3.61 Histograma e ACF do $\phi_{t}$ simulado, $\operatorname{RCINAR}(1) \ldots \ldots \ldots \ldots$. . . . . . . 48

3.62 Gráfico de uma série simulada, $\operatorname{RCINAR(1):~} E\left[\phi_{t}\right]=0,5$ e $\lambda=1 \ldots \ldots \ldots$. . . . 48

3.63 Histograma e boxplot da série simulada, $\operatorname{RCINAR}(1): E\left[\phi_{t}\right]=0,5$ e $\lambda=1 \ldots \ldots$. . . 48

3.64 Autocorrelação e Autocorrelação parcial amostrais $\operatorname{RCINAR(1):~} E\left[\phi_{t}\right]=0,5$ e $\lambda=1 . \quad 49$

3.65 Boxplot das estimativas de $E\left[\phi_{t}\right]$ para o modelo gerado por $X_{t}=\phi_{t} \circ X_{t-1}+I_{t} \ldots .49$

3.66 Boxplot das estimativas de $\lambda$ para o modelo gerado por $X_{t}=\phi_{t} \circ X_{t-1}+I_{t} \ldots \ldots$. . 49

3.67 Histograma alisado do viés de $\hat{\Phi}$ para o modelo gerado por $X_{t}=\phi_{t} \circ X_{t-1}+I_{t}$. . . 50

3.68 Histograma alisado do viés de $\hat{\lambda}$ para o modelo gerado por $X_{t}=\phi_{t} \circ X_{t-1}+I_{t}$. . . 50

3.69 Histograma e ACF do $\phi_{t}$ simulado, $\operatorname{RCINAR(1)\ldots \ldots \ldots \ldots ........~} 51$

3.70 Gráfico de uma série simulada, $\operatorname{RCINAR(1):~} E\left[\phi_{t}\right]=0,7$ e $\lambda=1 \ldots \ldots \ldots \ldots$

3.71 Histograma e boxplot da série simulada, $\operatorname{RCINAR}(1): E\left[\phi_{t}\right]=0,7$ e $\lambda=1 \ldots \ldots$. . 51

3.72 Autocorrelação e Autocorrelação parcial amostrais $\operatorname{RCINAR(1):~} E\left[\phi_{t}\right]=0,7$ e $\lambda=1$. 52

3.73 Boxplot das estimativas de $E\left[\phi_{t}\right]$ para o modelo gerado por $X_{t}=\phi_{t} \circ X_{t-1}+I_{t} \ldots . \quad 52$

3.74 Boxplot das estimativas de $\lambda$ para o modelo gerado por $X_{t}=\phi_{t} \circ X_{t-1}+I_{t} \ldots \ldots$. . 52

3.75 Histograma alisado do viés de $\hat{\Phi}$ para o modelo gerado por $X_{t}=\phi_{t} \circ X_{t-1}+I_{t}$. . . 53

3.76 Histograma alisado do viés de $\hat{\lambda}$ para o modelo gerado por $X_{t}=\phi_{t} \circ X_{t-1}+I_{t}$. . . 53

3.77 Histograma e ACF do $\phi_{1}^{(t)}$ simulado, $\operatorname{RCINAR}(2) \ldots \ldots \ldots \ldots \ldots$

3.78 Histograma e ACF do $\phi_{2}^{(t)}$ simulado, $\operatorname{RCINAR}(2) \ldots \ldots \ldots \ldots \ldots$

3.79 Gráfico de uma série simulada, $\operatorname{RCINAR}(2): E\left[\phi_{1}^{(t)}\right]=0,3, E\left[\phi_{2}^{(t)}\right]=0,3$ e $\lambda=1$. . 55

3.80 Histograma e boxplot da série simulada, $\operatorname{RCINAR}(2): E\left[\phi_{1}^{(t)}\right]=0,3, E\left[\phi_{2}^{(t)}\right]=0,3 \mathrm{e}$

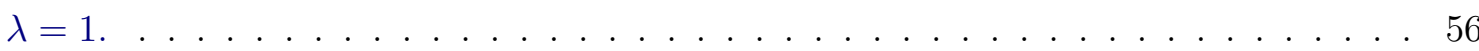

3.81 Autocorrelação e Autocorrelação parcial amostrais $\operatorname{RCINAR}(2): E\left[\phi_{1}^{(t)}\right]=0,3, E\left[\phi_{2}^{(t)}\right]=$

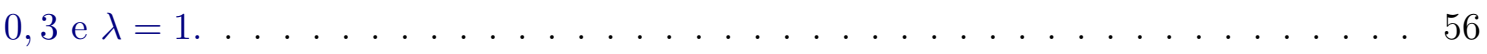

3.82 Boxplot das estimativas de $E\left[\phi_{1}^{(t)}\right]$ para o modelo gerado. . . . . . . . . . . . . 57

3.83 Boxplot das estimativas de $E\left[\phi_{2}^{(t)}\right]$ para o modelo gerado. . . . . . . . . . . . . 57

3.84 Boxplot das estimativas de $\lambda$ para o modelo gerado. . . . . . . . . . . . . . 57

3.85 Histograma alisado do viés de $\hat{\Phi}_{1}$ para o modelo gerado. . . . . . . . . . . . . 58

3.86 Histograma alisado do viés de $\hat{\Phi}_{2}$ para o modelo gerado. . . . . . . . . . . . . 58

3.87 Histograma alisado do viés de $\hat{\lambda}$ para o modelo gerado. . . . . . . . . . . . . . . . 58

3.88 Histograma e ACF do $\phi_{1}^{(t)}$ simulado, $\operatorname{RCINAR}(2) \ldots \ldots \ldots \ldots \ldots \ldots$

3.89 Histograma e ACF do $\phi_{2}^{(t)} \operatorname{simulado,~} \operatorname{RCINAR}(2) \ldots \ldots \ldots \ldots \ldots$. . . . . . . 59

3.90 Gráfico de uma série simulada, $\operatorname{RCINAR}(2): E\left[\phi_{1}^{(t)}\right]=0,3, E\left[\phi_{2}^{(t)}\right]=0,5$ e $\lambda=1$. . 60

3.91 Histograma e boxplot da série simulada, $\operatorname{RCINAR}(2): E\left[\phi_{1}^{(t)}\right]=0,3, E\left[\phi_{2}^{(t)}\right]=0,5 \mathrm{e}$ $\lambda=1$.

3.92 Autocorrelação e Autocorrelação parcial amostrais $\operatorname{RCINAR}(2): E\left[\phi_{1}^{(t)}\right]=0,3, E\left[\phi_{2}^{(t)}\right]=$ 0,5 e $\lambda=1$.

3.93 Boxplot das estimativas de $E\left[\phi_{1}^{(t)}\right]$ para o modelo gerado. . . . . . . . . . . . . 61

3.94 Boxplot das estimativas de $E\left[\phi_{2}^{(t)}\right]$ para o modelo gerado. . . . . . . . . . . . . 61

3.95 Boxplot das estimativas de $\lambda$ para o modelo gerado. . . . . . . . . . . . . . 61

3.96 Histograma alisado do viés de $\hat{\Phi}_{1}$ para o modelo gerado. . . . . . . . . . . . . . . 62 
3.97 Histograma alisado do viés de $\hat{\Phi}_{2}$ para o modelo gerado. . . . . . . . . . . . . . . . 62

3.98 Histograma alisado do viés de $\hat{\lambda}$ para o modelo gerado. . . . . . . . . . . . . . . . 62

3.99 Histograma e ACF do $\phi_{1}^{(t)}$ simulado, $\operatorname{RCINAR}(2) \ldots \ldots \ldots \ldots \ldots \ldots$

3.100 Histograma e ACF do $\phi_{2}^{(t)}$ simulado, RCINAR(2) . . . . . . . . . . . . 63

3.101Gráfico de uma série simulada, $\operatorname{RCINAR}(2): E\left[\phi_{1}^{(t)}\right]=0,5, E\left[\phi_{2}^{(t)}\right]=0,3$ e $\lambda=1$. . 63

3.102Histograma e boxplot da série simulada, $\operatorname{RCINAR}(2): E\left[\phi_{1}^{(t)}\right]=0,5, E\left[\phi_{2}^{(t)}\right]=0,3$ e

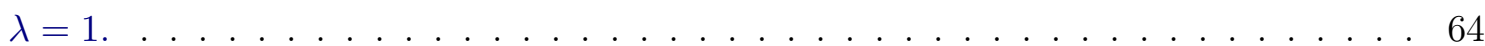

3.103Autocorrelação e Autocorrelação Parcial Amostrais $\operatorname{RCINAR}(2): E\left[\phi_{1}^{(t)}\right]=0,5, E\left[\phi_{2}^{(t)}\right]=$

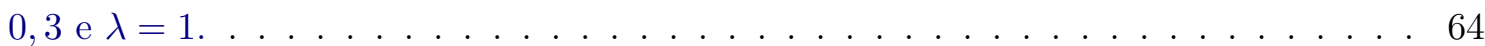

3.104 Boxplot das estimativas de $E\left[\phi_{1}^{(t)}\right]$ para o modelo gerado. . . . . . . . . . . . 65

3.105Boxplot das estimativas de $E\left[\phi_{2}^{(t)}\right]$ para o modelo gerado. . . . . . . . . . . . . . 65

3.106Boxplot das estimativas de $\lambda$ para o modelo gerado. . . . . . . . . . . . . . 65

3.107Histograma alisado do viés de $\hat{\Phi}_{1}$ para o modelo gerado. . . . . . . . . . . . . . . 66

3.108Histograma alisado do viés de $\hat{\Phi}_{2}$ para o modelo gerado. . . . . . . . . . . . . . 66

3.109Histograma alisado do viés de $\hat{\lambda}$ para o modelo gerado. . . . . . . . . . . . . . . 66

4.1 Número de falências decretadas - Médias empresas. . . . . . . . . . . . . . . . . . . 70

4.2 Histograma e boxplot do número de falência decretada - Médias empresas. . . . . . . 70

4.3 Autocorrelação e autocorrelação parcial do número de falência decretada - Médias empresas. . . . . . . . . . . . . . . . . . . . . 70

4.4 Série real e ajuste do modelo $\operatorname{RCINAR}(1) \ldots \ldots \ldots \ldots \ldots$. . . . . . . . . . 71

4.5 Histograma e boxplot do resíduo do ajuste. . . . . . . . . . . . . . . . . 71

4.6 Autocorrelação e autocorrelação parcial do resíduo do ajuste. . . . . . . . . . . . . . 71

4.7 Número de consultas. . . . . . . . . . . . . . . . . . . . . . . . . . . . 73

4.8 Histograma e boxplot da quantidade de consultas. . . . . . . . . . . . . . . . . . . 73

4.9 Autocorrelação e autocorrelação parcial da quantidade de consultas. . . . . . . . . . . 74

4.10 Série real e ajuste do modelo $\operatorname{RCINAR}(2) \ldots \ldots \ldots \ldots$

4.11 Histograma e boxplot do resíduo do ajuste. . . . . . . . . . . . . . . . . . . 75

4.12 Autocorrelação e autocorrelação parcial do resíduo do ajuste. . . . . . . . . . . . . 75 


\section{Lista de Tabelas}

3.1 Modelos de referência. . . . . . . . . . . . . . . . . . . . . . . 12

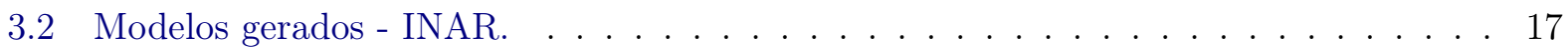

3.3 Viés das estimações para o modelo $\operatorname{INAR}(1)(\lambda=1)$ baseado em 500 réplicas. . . . . 27

3.4 Erro Quadrático Médio das estimações para o modelo $\operatorname{INAR}(1)(\lambda=1)$ baseado em 500 réplicas. . . . . . . . . . . . . . . . . . . . . . . . 27

3.5 Variância dos valores estimados para o modelo $\operatorname{INAR}(1)(\lambda=1)$ baseado em 500 réplicas. . . . . . . . . . . . . . . . . . . . . . 28

3.6 Viés das estimativas dos parâmetros do modelo $\operatorname{INAR}(2)(\lambda=1)$ baseado em 500 réplicas e $\mathrm{N}=200 \ldots \ldots \ldots \ldots$

3.7 EQM das estimativas dos parâmetros do modelo $\operatorname{INAR}(2)(\lambda=1)$ baseado em 500

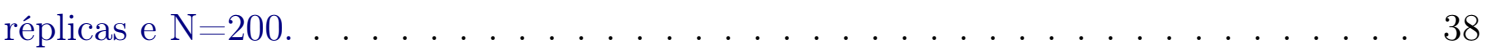

3.8 Modelos gerados - RCINAR. . . . . . . . . . . . . . . . . . . 44

3.9 Viés das estimações para o modelo $\operatorname{RCINAR}(1)(\lambda=1)$ baseado em 500 réplicas. . . 54

3.10 Erro Quadrático Médio das estimações para o modelo $\operatorname{RCINAR}(1)(\lambda=1)$ baseado em 500 réplicas. . . . . . . . . . . . . . . . . . . . . 54

3.11 Viés das estimativas dos parâmetros do modelo $\operatorname{RCINAR}(2)(\lambda=1)$ baseado em 500 réplicas e $\mathrm{N}=200 \ldots \ldots \ldots$. . . . . . . . . . . . . . . . . . . 67

3.12 EQM das estimativas dos parâmetros do modelo $\operatorname{RCINAR}(2)(\lambda=1)$ baseado em

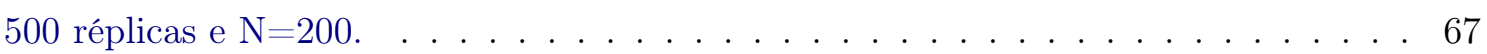

4.1 Comparativo da previsão. . . . . . . . . . . . . . . . . . . . . . 72 


\section{Capítulo 1}

\section{Introdução}

\subsection{Considerações Preliminares}

Metodologias de séries temporais tem ganhado grande espaço no mercado; isso ocorre porque as companhias têm cada vez mais claro a necessidade de que suas estratégias sejam monitoradas através de indicadores internos; além disso, o monitoramento do mercado que ela está inserida. A antecipação de movimentos do mercado traz inúmeros benefícios. A identificação da tendência de crescimento populacional numa determinada região ajudará no planejamento de políticas públicas, na penetração de determinada rede de supermercado. Exemplos desse tipo são clássicos como justificativa do uso de modelos preditivos.

O uso de modelos de séries temporais só é possível quando existe uma quantidade razoável de mensurações em diversos intantes de tempo. Há alguns anos essa preocupação está sendo alvo das empresas que investem em mais recursos de tecnologia para armazenamento da informação histórica.

Quando nos concentramos em modelos de séries temporais, temos em conta que a análise para resolução de um problema pode seguir distintos caminhos de acordo com a necessidade do estudo. Antecipar valores de índices dos próximos anos ou simplesmente entender o que causa a oscilação do índice são exemplos de diferentes formas de uso do modelo de séries. Os objetivos comuns de utilização de modelos de séries temporais são:

- Compreensão do comportamento das variáveis. Em geral esse tipo de estudo visa interpretar a elasticidade da variável resposta em relação às variáveis explicativas, sejam em tempos coincidentes ou defasados no tempo.

- Previsão. Talvez esse seja o objetivo mais citado no mercado. Realizar projeção de uma variável auxilia as empresas no dimensionamento dos seus negócios e no planejamento de estratégias.

\subsection{Objetivos}

Este estudo assume como principal objetivo o entendimento de processos de séries temporais que não são muito comuns, os processos autorregressivos inteiros. Entender como é o comportamento desse processo, quais suas características e como utilizá-lo na prática com problemas reais também são foco deste trabalho. 


\subsection{Contribuições}

As principais contribuições que este trabalho traz, são:

1. Estudo de um modelo não comum para práticas de previsão no mercado.

2. Comparação da performance de diferentes métodos de estimação.

3. Avaliação de séries temporais reais com os modelos propostos.

4. Aplicação de uma metodologia de séries temporais como variável de entrada para um modelo de risco de crédito.

\subsection{Organização do Trabalho}

O Capítulo 2 apresenta conceitos iniciais para o estudo dos modelos propostos; os processos de séries temporais inteiras, bem como suas propriedades, métodos de estimação, estudo de simulação e previsão são apresentados no Capítulo 3. Ao final, o Capítulo 4 traz a aplicação dos modelos propostos a dados reais. 


\section{Capítulo 2}

\section{Conceitos}

\subsection{Séries Temporais}

A observação de uma variável aleatória (v.a.) tomada sequencialmente em diferentes instantes do tempo é chamada de série temporal. Seja $T$ um conjunto arbitrário e $X \in \mathbb{R}$; uma série temporal é denotada por $\left\{X_{t}\right\}_{t \in T}$.

Séries temporais podem se diferenciar pelo tipo da variável $X$ ou pela característica do indexador de tempo $T$. Normalmente o conjunto $T$ é tomado como o conjunto dos inteiros mas também existem séries onde o indexador pertence ao conjunto dos reais, é o caso de observações de aparelhos medidores de nível do mar, por exemplo.

A série temporal pode ser classificada como contínua ou discreta, dependendo do evento que se observa ao longo do tempo. A taxa de juros, o valor vendido de um determinado produto são exemplos de séries contínuas, já a quantidade de ocorrência de raios num local durante um determinado tempo ou o número de reparos numa central de distribuição elétrica são classificados como séries discretas.

Este trabalho contemplará séries temporais discretas, em específico, séries de observações inteiras. Essas séries em geral tratam de contagem de eventos; o estudo desse tipo de série temporal incorpora a análise de modelos não muito comuns chamados de INAR (Integer Valued Autoregressive Process).

\subsection{Estacionariedade}

De maneira simples, uma série temporal é dita estacionária quando ela é invariante sobre translações no tempo, mantendo o equilíbrio estatístico e suas características probabilísticas. Podemos definir dois tipos de estacionariedade, fraca (ou ampla ou de segunda ordem) e estrita (ou forte).

Definição - Estacionariedade forte: A série é classificada como estritamente estacionária quando a distribuição de probabilidade multivariada permanece a mesma sob translações no tempo. O comportamento para todos valores

$$
\left\{x_{t_{1}}, x_{t_{2}}, x_{t_{3}}, \ldots, x_{t_{k}}\right\},
$$

é idêntico quando há translação no tempo

$$
\left\{x_{t_{1}+h}, x_{t_{2}+h}, x_{t_{3}+h}, \ldots, x_{t_{k}+h}\right\}
$$

Assim,

$$
\mathrm{P}\left\{x_{t_{1}} \leq c_{1}, \ldots, x_{t_{k}} \leq c_{k}\right\}=\mathrm{P}\left\{x_{t_{1}+h} \leq c_{1}, \ldots, x_{t_{k}+h} \leq c_{k}\right\},
$$

para todo $k=1,2, \ldots$, todos pontos no tempo $t_{1}, t_{2}, \ldots, t_{k}$, todos números $c_{1}, c_{2}, \ldots, c_{k}$ e toda translação $h=0, \pm 1, \pm 2, \ldots$. 
Se uma série é estritamente estacionária, então todo subconjunto das variáveis também possuem a mesma característica estacionária, para qualquer valor de k. Em particular o caso unidimensional, que apresenta sua média $\mu(t)$ e variância $V(t)$ constantes,

$$
\mu(t)=\mu, V(t)=\sigma^{2}
$$

Definição - Estacionariedade fraca: O processo $\left\{X_{t}\right\}_{t \in \mathbb{Z}}$ é dito fracamente estacionário se e somente se:

(i) $E\left(X_{t}\right)=\mu(t)=\mu$

(ii) $E\left(X_{t}^{2}\right)<\infty$

(iii) $\gamma\left(t_{1}, t_{2}\right)=\operatorname{Cov}\left(X_{t_{1}}, X_{t_{2}}\right)$ é função somente da diferença $\left|t_{1}-t_{2}\right|$.

Um processo que satisfaz (ii) é chamado de processo de segunda ordem. A notação de série estacionária tratada nesse trabalho se refere a estacionariedade do tipo fraca.

\subsubsection{Função de autocorrelação}

Seja $\left\{X_{t}\right\}_{t \in \mathbb{Z}}$ um processo estacionário, sua autocovariância é definida como

$$
\gamma(h)=E\left[\left(x_{t+h}-\mu\right)\left(x_{t}-\mu\right)\right]
$$

e a autocorrelação (ACF), como função de (2.1), é dada por

$$
\rho(h)=\frac{\gamma(h)}{\gamma(0)},
$$

com as seguintes propriedades:

(i) $-1 \leq \rho(h) \leq 1$,

(ii) $\rho(h)=\rho(-h)$.

A autocorrelação mede a dependência linear da série com ela mesma deslocada no tempo.

\subsubsection{Função de autocorrelação parcial}

Um processo estacionário possui autocorrelação parcial (PACF) denotada por $\phi_{h h}$ para $h=$ $1,2, \ldots$ definida como o último coeficiente do ajuste de um modelo $\mathrm{AR}(\mathrm{h})$ ao processo.

A autocorrelação parcial pode ser calculada em função das autocorrelações, como o quociente de dois determinantes.

$$
\phi_{h h}=\frac{\left|\begin{array}{ccccc}
1 & \rho(1) & \rho(2) & \cdots & \rho(1) \\
\rho(1) & 1 & \rho(2) & \cdots & \rho(2) \\
\rho(2) & \rho(2) & 1 & \cdots & \rho(3) \\
\vdots & \vdots & \vdots & \ddots & \vdots \\
\rho(h-1) & \rho(h-2) & 1 & \cdots & \rho(h)
\end{array}\right|}{\left|\begin{array}{ccccc}
1 & \rho(1) & \rho(2) & \cdots & \rho(h-1) \\
\rho(1) & 1 & \rho(2) & \cdots & \rho(h-2) \\
\rho(2) & \rho(2) & 1 & \cdots & \rho(h-3) \\
\vdots & \vdots & \vdots & \ddots & \vdots \\
\rho(h-1) & \rho(h-2) & 1 & \cdots & 1
\end{array}\right|} .
$$




\subsection{Modelo Autorregressivo}

Os modelos autorregressivos merecem uma atenção especial neste trabalho pois é um modelo em que o padrão de autocorrelação é considerado como referência para os demais a serem estudados aqui. Tratam-se de modelos pertencentes à classe de modelos lineares estacionários. Um modelo autorregressivo de ordem $p$ é definido como o processo $\left\{X_{t}\right\}_{t \in T}$ tal que

$$
\begin{aligned}
X_{t} & =\phi_{1} X_{t-1}+\phi_{2} X_{t-2}+\cdots+\phi_{p} X_{t-p}+\epsilon_{t} \\
& =\sum_{j=1}^{p} \phi_{i} X_{t-i}+\epsilon_{t}
\end{aligned}
$$

em que $p \in \mathbb{Z}$ e $\epsilon_{t}$ é ruído branco com média $\mu=0$ e variância $\sigma^{2}$.

A estacionariedade de um modelo autorregressivo de ordem $p$ é facilmente verificada quando se transforma a equação (2.4) em função da soma infinita de $\epsilon_{t}$. Considerando o modelo (2.4) verificamos que $X_{t}$ pode ser representado como

$$
\begin{aligned}
X_{t} & =\frac{\epsilon_{t}}{\left(1-\phi_{1} B-\phi_{2} B^{2}-\cdots-\phi_{p} B^{p}\right)} \\
& =\sum_{j=0}^{\infty} \psi_{j} \epsilon_{t-j} .
\end{aligned}
$$

A igualdade (2.5) é verificada considerando as raízes do polinômio $\left(1-\phi_{1} B-\phi_{2} B^{2}-\cdots-\phi_{p} B^{p}\right)$ fora do círculo unitário. Através da representação da soma infinita de $\epsilon$ nota-se que a esperança, a variância e a autocorrelação da variável aleatória $X_{t}$ não dependem do tempo, seguindo as predisposições de estacionariedade; para uma melhor compreensão veja (Morettin e Toloi, 2006).

\subsubsection{Propriedades do modelo autorregressivo - AR(p)}

O modelo AR pode ser caracterizado pelo comportamento da sua autocorrelação e autocorrelação parcial. Seja o modelo dado em (2.4); as seguintes propriedades são observadas:

- $E\left(X_{t}\right)=0$

- $\operatorname{Var}\left(X_{t}\right)=\frac{\sigma^{2}}{\sum_{j=1}^{p} \phi_{j} \rho(k-j)}$

- $\operatorname{Cov}\left(X_{t}, X_{t-k}\right)=\gamma(k)=\sum_{j=1}^{p} \phi_{j} \gamma(k-j)$

- $\operatorname{Cor}\left(X_{t}, X_{t-k}\right)=\rho(k)=\frac{\gamma(k)}{\gamma(0)}=\sum_{j=1}^{p} \phi_{j} \rho(k-j)$.

Exemplo: A Figura 2.1 apresenta o gráfico de um modelo $\mathrm{AR}(1)$ com $\phi=0,74$, tomando $\epsilon_{t}$ normalmente distribuído com média $\mu=0$ e variância $\sigma^{2}=3$. 


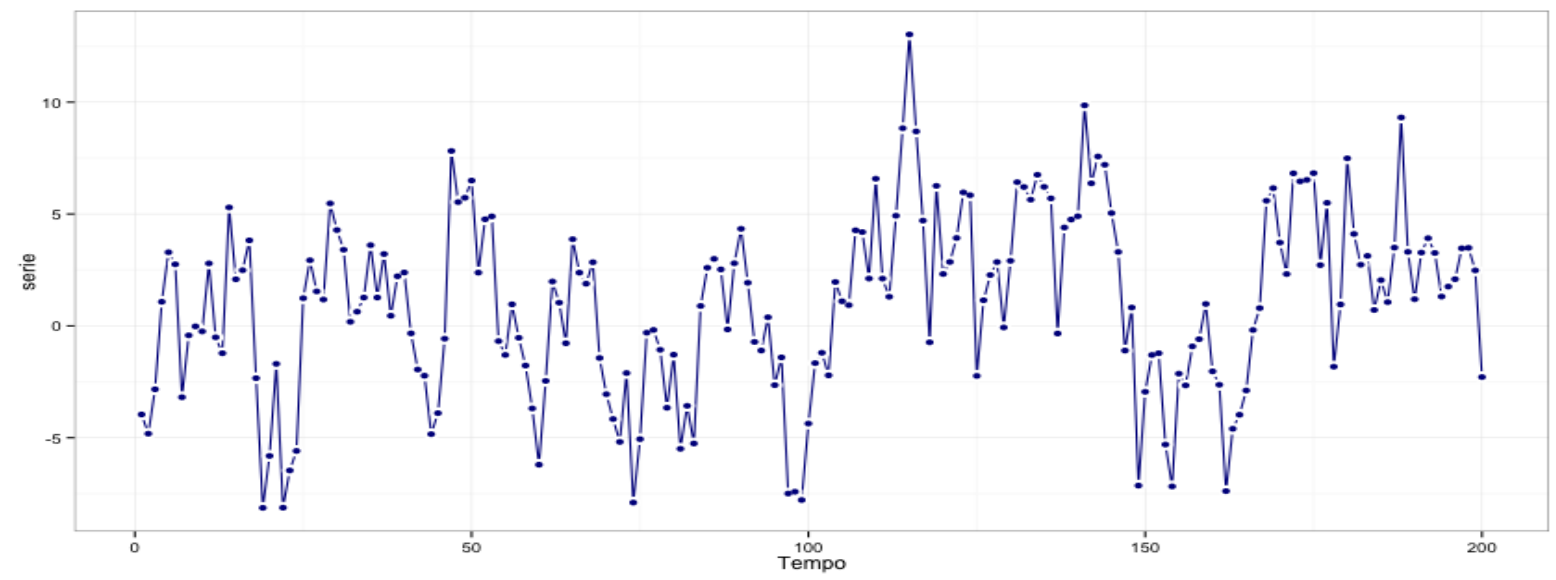

Figura 2.1: Série simulada - AR(1) $\operatorname{com} \phi_{1}=0,74$

Nota-se que a série tem os valores observados ao redor da média zero, com variância constante. Já a Figura 2.2 mostra o comportamento da autocorrelação e autocorrelação parcial da série gerada; tal autocorrelação apresenta decaimento exponencial e a autocorrelação parcial tem como única defasagem (lag) significante, a de ordem 1 de acordo com o esperado pelas propriedades do modelo AR(1) e mostrado na Figura 2.3.
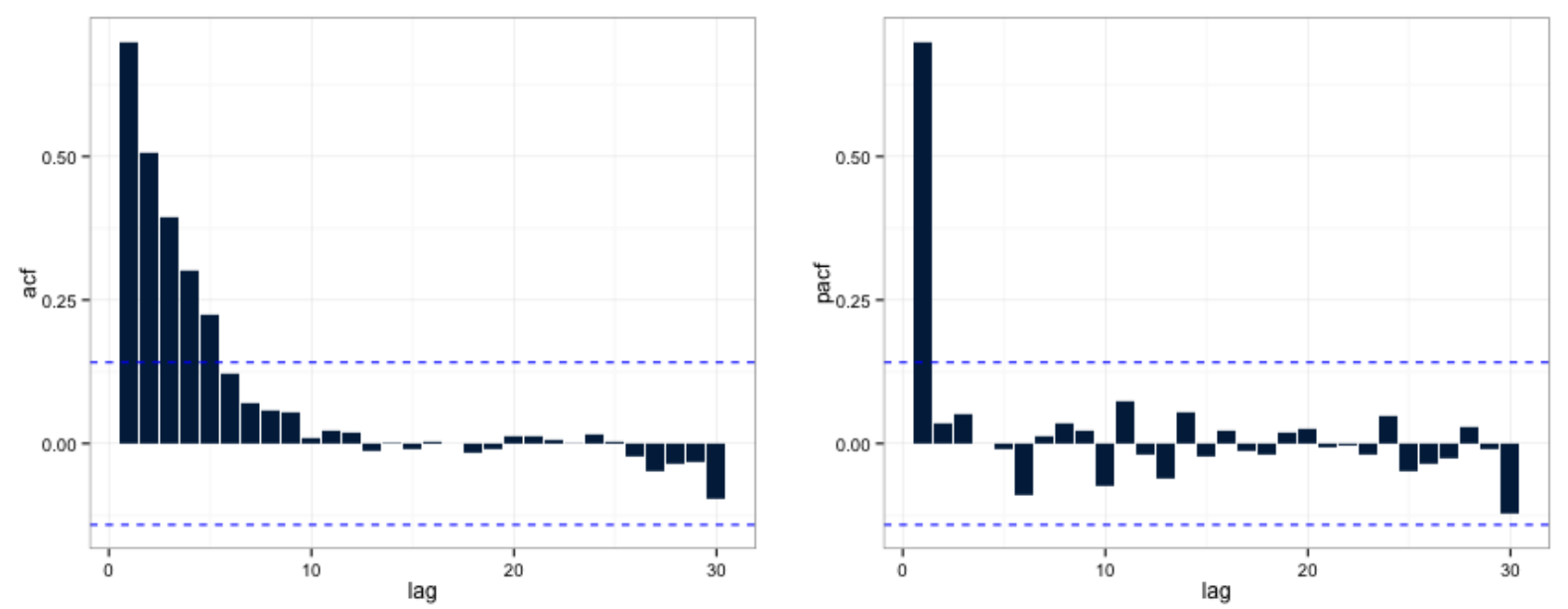

Figura 2.2: Autocorrelação e Autocorrelação parcial amostral de um modelo AR(1) gerado com $\phi_{1}=0,74$

O gráfico de autocorrelação e autocorrelação parcial é acompanhado dos limites de confiança, traçado em $+/-2$ vezes o desvio padrão, calculado como $\frac{1}{\sqrt{N}}$ em que $N$ é o tamanho observado da série. 

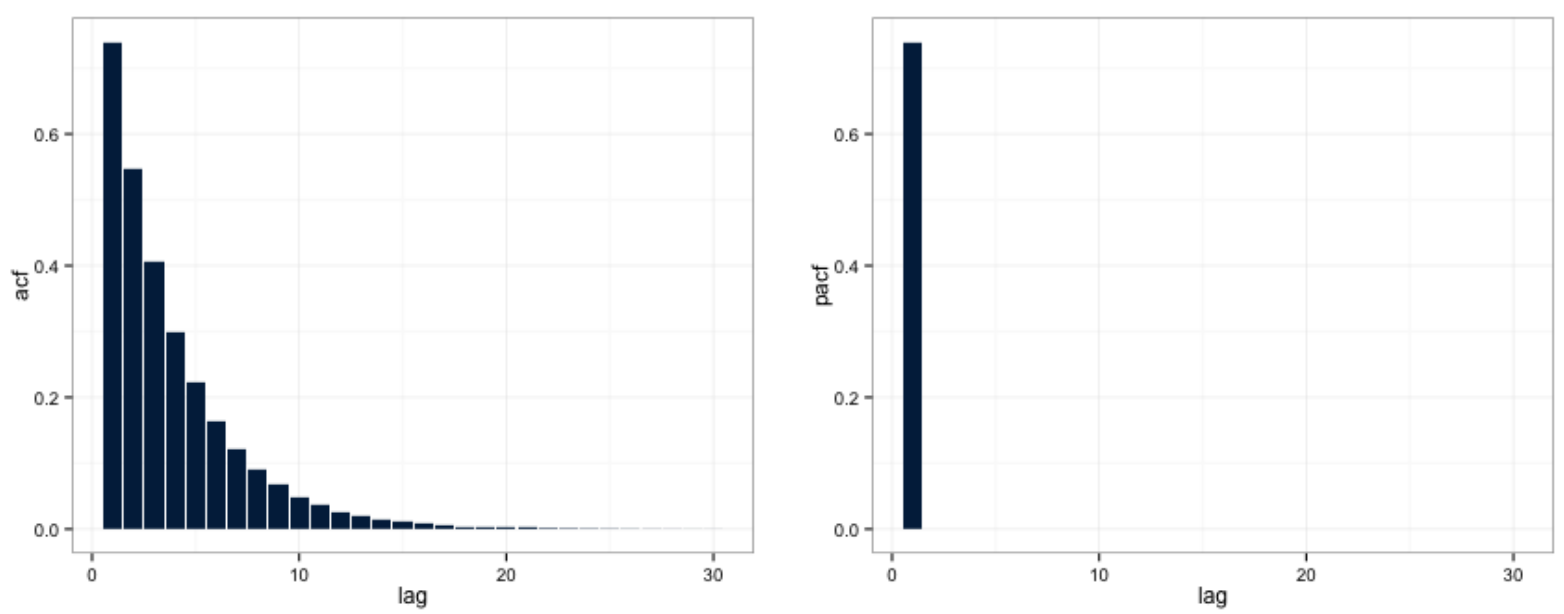

Figura 2.3: Autocorrelação e Autocorrelação parcial teóricas do modelo AR(1) com $\phi_{1}=0,74$

\subsection{Medida de acurácia}

Existem distintas metodologias para medir a adequação de um ajuste de séries temporais. Apresenta-se duas medidas comumente usadas, o EQM (Erro Quadrático Médio) e o viés. Este trabalho trata a qualidade de ajuste de um método de estimação baseado nessas duas medidas.

O Erro Quadrático Médio denota a distancia média entre o valor observado e estimado ao quadrado, sendo como:

$$
E Q M=\frac{1}{N} \sum_{j=1}^{N}(\text { erro })^{2}=\frac{1}{N} \sum_{j=1}^{N}\left(\hat{Y}_{j}-Y_{j}\right)^{2}
$$

O viés de estimação do parâmetro mede quão próximo a estimativa encontra-se do verdadeiro valor do parâmetro $(\phi)$; o viés é definido como:

$$
\operatorname{vies}(\hat{\phi})=\phi-E[\hat{\phi}]
$$




\subsection{Operador Thinning Binomial}

Nessa seção será apresentado o operador Thinning Binomial pertencente a classe de operadores Thinning, proposto por Steutel e Harn (1979).

Definição: Seja $\alpha \in \mathbb{R}$, onde $0 \leq \alpha \leq 1$ e $X$ uma variável aleatória inteira não nula.

O operador Thinning Binomial é dado por:

$$
\alpha \circ X=\sum_{i=1}^{X} U_{i},
$$

em que $\left\{U_{i}\right\}_{i=1}^{X}$ é uma sequência de variáveis aleatórias independentes e identicamente distribuídas (i.i.d.) tais que $P\left(U_{i}=1\right)=1-P\left(U_{i}=0\right)=\alpha$. Logo a sequência aleatória $\left\{U_{i}\right\}_{i=1}^{X}$ tem distribuição Bernoulli.

O operador Binomial Thinning possui uma forte interpretação na prática pois representa a parte do valor observado no passado que continuará sendo observado.Num problema de imigração, o Binomial Thinning representa o número de pessoas remanecentes de um instante de tempo para outro, que não migraram; por sua vez a probabilidade $\alpha$ indica a chance de cada pessoa não migrar, isto é, a taxa com que os valores em $t-1$ 'sobrevivam' até $t$.

Definindo $\alpha_{i} \in[0,1]$ para $i=1,2$ e $X_{1}, X_{2} \in \mathbb{N}^{+}$, o operador Thinning Binomial dado em (2.8) possui as seguintes propriedades:

- $0 \circ X=0$

- $1 \circ X=X$

- $\alpha_{1} \circ\left(\alpha_{2} \circ X\right)=\left(\alpha_{1} \alpha_{2}\right) \circ X$

- $\alpha_{1} \circ\left(X_{1}+X_{2}\right)=\alpha_{1} \circ X_{1}+\alpha_{1} \circ X_{2}$

- $E\left(\alpha_{1} \circ X\right)=\alpha_{1} E(X)$

- $E\left(\left(\alpha_{1} \circ X_{1}\right) X_{2}\right)=\alpha_{1} E\left(X_{1} X_{2}\right)$

- $\left(\alpha_{1} \circ X\right)$ possui distribuição $\operatorname{Binomial}\left(X, \alpha_{1}\right)$

- $\operatorname{Var}\left(\alpha_{1} \circ X\right)=\alpha_{1}^{2} \operatorname{Var}(X)+\alpha_{1}\left(1-\alpha_{1}\right) E(X)$

- $\operatorname{Cov}\left(\alpha_{1} \circ X, \alpha_{2} \circ Y\right)=\alpha_{1} \alpha_{2} \operatorname{Cov}(X, Y)$. 


\section{Capítulo 3}

\section{Modelos autorregressivos inteiros}

A pesquisa por modelos para séries temporais inteiras estacionárias tem espaço no meio acadêmico há mais de três décadas. Jacobs e Lewis (1983) introduziram o modelo DARMA (Discrete Autoregressive Moving Average models) dado por uma mistura de sequências de variáveis aleatórias discretas. Como exemplo desse modelo consideremos o modelo DARMA $(1,1)$ definido a seguir.

$$
X_{t}= \begin{cases}Y_{t} & \text { com probabilidade } \beta \\ A_{t-1} & \text { com probabilidade }(1-\beta)\end{cases}
$$

em que

$$
A_{t-1}= \begin{cases}A_{t-2} & \text { com probabilidade } \rho \\ Y_{t-1} & \text { com probabilidade }(1-\rho)\end{cases}
$$

O modelo DARMA possui uma escolha aleatória entre as sequências $A_{t-1}$ e $Y_{t}$. Quando $Y_{t}$ é tomado, o passado da série é desprezado, o que contradiz propriedades de memória de séries temporais que decai exponencialmente (Box et al., 2008). Tendo conhecimento dessa contradição sobre os modelos DARMA, Al-Osh e Alzaid (1987) propuseram um modelo para séries de valores inteiros de forma análoga aos modelos tradicionais AR, dando origem aos modelos INAR(1) Integer Autoregressive model de ordem um. Alguns anos mais tarde, seguindo a linha de modelos para séries temporais inteiras, Alzaid e Al-Osh (1990) propuseram os modelos INAR(p). A pesquisa da classe de modelos para séries inteiras não negativas evoluiu e foi introduzido um novo conceito de parâmetros. Jin-Guan e Li-Yuan (1991) buscaram modelos em que o parâmetro do modelo também possui comportamento aleatório variando no tempo. É o caso do modelo RCINAR - Random Coefficient Integer Autoregressive model.

No mercado, modelos de contagem são muito utilizados em problemas de comportamento de compra, em que a quantidade de venda de um produto segue uma distribuição discreta, além disso, trata-se de uma variável inteira não negativa, foco da classe de modelos INAR e RCINAR. Outra aplicação bastante encontrada diz respeito ao problema de migração, que trata a série de número de pessoas residentes num local determinado. 


\subsection{Modelos INAR}

O modelo INAR (Integer valued Autoregressive process), como mencionado, é um processo de contagem que considera variáveis inteiras não negativas. O primeiro modelo INAR proposto por Al-Osh e Alzaid (1987), considera somente a informação do momento imediatamente anterior, assim como os modelos autorregressivos de ordem 1. Não necessariamente toda a informação de uma série temporal pode ser capturada somente através da última observação; com o avanço computacional e a necessidade de modelos mais robustos quanto à estrutura do histórico, Alzaid e Al-Osh (1990) e Jin-Guan e Li-Yuan (1991) investigaram modelos com maior complexidade, analogamente aos modelos $\mathrm{AR}(\mathrm{p})$, definindo o modelo genérico INAR de ordem $p$.

O modelo INAR é composto por duas parcelas, definido como segue:

$$
X_{t}=C_{t-1}+I_{t}
$$

em que

- $C_{t-1}=\alpha \circ X_{t-1}$ é responsável pela captura de toda informação que o histórico traz. É função da soma de operadores Binomial Thinning calculados com fontes em diferentes pontos no tempo até $t$-1, inclusive. Cada operador Binomial Thinning possui uma probabilidade associada, que é um parâmetro a ser estimado. Quando pensamos no processo de comportamento de compra ou venda, $C_{t-1}$ é interpretado como consumidores remanescentes dado o último conjunto de compradores, sendo a parte da série temporal que continua sendo observada com uma probabilidade associada $\alpha, \alpha \in(0,1)$ ou habitantes remanescentes em uma região no caso de migração.

- $I_{t}$ é uma variável aleatória discreta não negativa com média $\mu_{I}$ e variância $\sigma_{I}^{2}$ independente de $C_{t-1}$. Representa a inovação em $t$, uma parte não correlacionada com o passado. Mckenzie(1988), Al-Osh e Alzaid (1987) propuseram modelos com a inovação seguindo a distribuição Poisson, mas as distribuições Geométrica ou Binomial Negativa também podem ser consideradas como sugerido por Mckenzie(1986). A inovação em $t$ é interpretada como novos clientes ou novas compras num processo de compra e venda, ou como novos habitantes de uma região num problema de migração.

O modelo INAR pode ser diferenciado pela memória temporal que carrega ou pela distribuição marginal considerada. Este trabalho tratará principalmente o modelo INAR com distribuição Poisson, e com memória de alcance variável de ordem p.

A abordagem tratada nesse estudo considera as inovações $I_{t}$ dadas pela distribuição de Poisson. Essa escolha é devido à existência de maior número de trabalhos direcionados à essa distribuição, que parece ser uma distribuição razoável para aplicação sobre uma série de quantidade de consulta a uma empresa. 


\subsubsection{Modelo INAR(1)}

Análogo ao modelo autorregressivo de ordem 1, AR(1), o modelo INAR(1) é expresso como função da última observação da série, apresentado como:

$$
X_{t}=\alpha \circ X_{t-1}+I_{t},
$$

em que $I_{t}$ variável aleatória discreta $\operatorname{Poisson}(\lambda)$ i.i.d. que representa a inovação enquanto o operador Binomial Thinning de ordem um representa a dependência da variável no último instante. Considerando as propriedades do operador Binomial Thinning, apresentadas no Capítulo 2.5 e o modelo estacionário $(3.2)$ com $\alpha \in(0,1)$, temos

- $E\left(X_{t}\right)=E\left(\alpha \circ X_{t-1}+I_{t}\right)=\frac{\lambda}{1-\alpha}$

- $\operatorname{Var}\left(X_{t}\right)=\gamma(0)=\operatorname{Var}\left(\alpha \circ X_{t-1}+I_{t}\right)=\frac{\lambda(1-\alpha)}{\left(1-\alpha^{2}\right)}$

- $\operatorname{Cov}\left(X_{t}, X_{t-k}\right)=\gamma(k)=\alpha^{k} \gamma(0)=\alpha^{k} \frac{\lambda(1-\alpha)}{\left(1-\alpha^{2}\right)}$

- $\operatorname{Cor}\left(X_{t}, X_{t-k}\right)=\rho(k)=\frac{\gamma(k)}{\gamma(0)}=\alpha^{k}$

- $\phi_{k k}=\alpha^{k}, \mathrm{k}=1$ e $\phi_{k k}=0, \mathrm{k}>1$

A autocorrelação e autocorrelação parcial caracterizam a série em relação ao tipo de estrutura de dependência que ela possui. O modelo INAR não podia ser diferente, cada processo possui um comportamento idiossincrático da autocorrelação e autocorrelação parcial, essas características são identificadas usando a comparação das autocorrelações teóricas e amostrais. Como apresentado, a autocorrelação de ordem $k$ de um processo INAR(1) é dada por $\rho(k)=\alpha^{k}$.

Exemplo: Seja $\alpha=0,35$ temos $\rho(k)=0,35^{k}, \phi_{k k}=0,35^{k}$ para $\mathrm{k}=1$ e $\phi_{k k}=0, \mathrm{k} \neq 1$. A Figura 3.1 mostra o comportamento esperado da autocorrelação e autocorrelação parcial do modelo $\operatorname{INAR}(1) \operatorname{com} \alpha=0,35$ e $\lambda=20$.
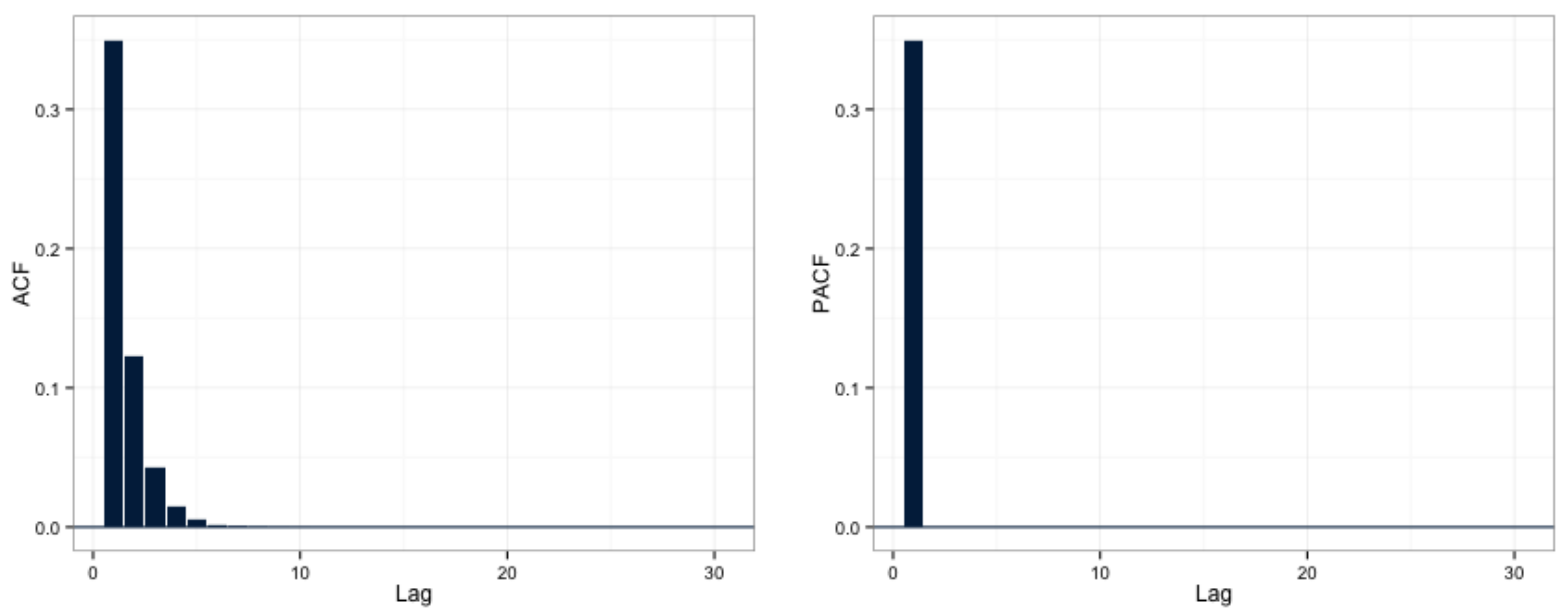

Figura 3.1: Autocorrelação e Autocorrelação parcial teórica: $\operatorname{INAR(1)}$ com $\alpha=0,35$ e $\lambda=20$. 


\subsubsection{Modelo INAR(p)}

A generalização do modelo INAR, representado por $\operatorname{INAR}(\mathrm{p})$, é análoga ao modelo AR(p). A classe dos modelos INAR(p) considera os $p$ últimos valores como fatores determinantes para o valor da série no tempo atual. O modelo $\operatorname{INAR}(\mathrm{p})$ é representado da seguinte forma:

$$
X_{t}=\sum_{j=1}^{p} \alpha_{j} \circ X_{t-j}+I_{t} .
$$

No modelo dado em (3.3), $I_{t}$ é uma variável aleatória i.i.d. Poisson $(\lambda)$ e $C_{t-1}$ a combinação linear dos operadores Binomial Thinning em distintas ordens de defasagens. Cada ordem de defasagem $\mathrm{k}$ possui uma probabilidade $\alpha_{k}$ associada, $k=1,2, \cdots, p$. O modelo (3.3) pode ser decomposto assim como apresentado em (3.1), considerando a parte da inovação $I_{t}$ e o componente $C_{t-1}$ que representa a combinação linear de operadores Binomial Thinning em diferentes ordens.

As propriedades do INAR(p) seguem como generalização do modelo com ordem restrita em um:

- $E\left(X_{t}\right)=E\left(\sum_{j=1}^{p} \alpha_{j} \circ X_{t-j}+I_{t}\right)=\frac{\lambda}{1-\sum_{j=1}^{p} \alpha_{j}}, \sum_{j=1}^{p} \alpha_{j}<1$;

- $\operatorname{Cov}\left(X_{t}, X_{t-k}\right)=\gamma(k)=\sum_{j=1}^{p} \alpha_{j} \gamma(k-j)+\delta_{k}(0) \lambda$;

- $\operatorname{Cor}\left(X_{t}, X_{t-k}\right)=\rho(k)=\frac{\gamma(k)}{\gamma(0)}=\sum_{j=1}^{p} \alpha_{j} \rho(k-j)$,

em que $\delta_{k}(x)=1$ se $x=k$ e zero caso contrário.

Partindo de modelos de ordem 2 e 5 , com o coeficiente de inovação $\lambda$ e as probabilidades $\alpha_{j}$ como mostrado na Tabela 3.1, calcula-se a autocorrelação e autocorrelação parcial, apresentadas na Figura 3.2 para o modelo de ordem 2 e na Figura 3.3 para o modelo de ordem 5.

\begin{tabular}{ccc}
\hline \hline Modelo & $\lambda$ & Parâmetros $\alpha_{i}$ \\
\hline INAR $(2)$ & 3 & 0,$3 ; 0,18$ \\
INAR(5) & 1 & 0,$22 ; 0,15 ; 0,2 ; 0,08 ; 0,1$ \\
\hline \hline
\end{tabular}

Tabela 3.1: Modelos de referência.
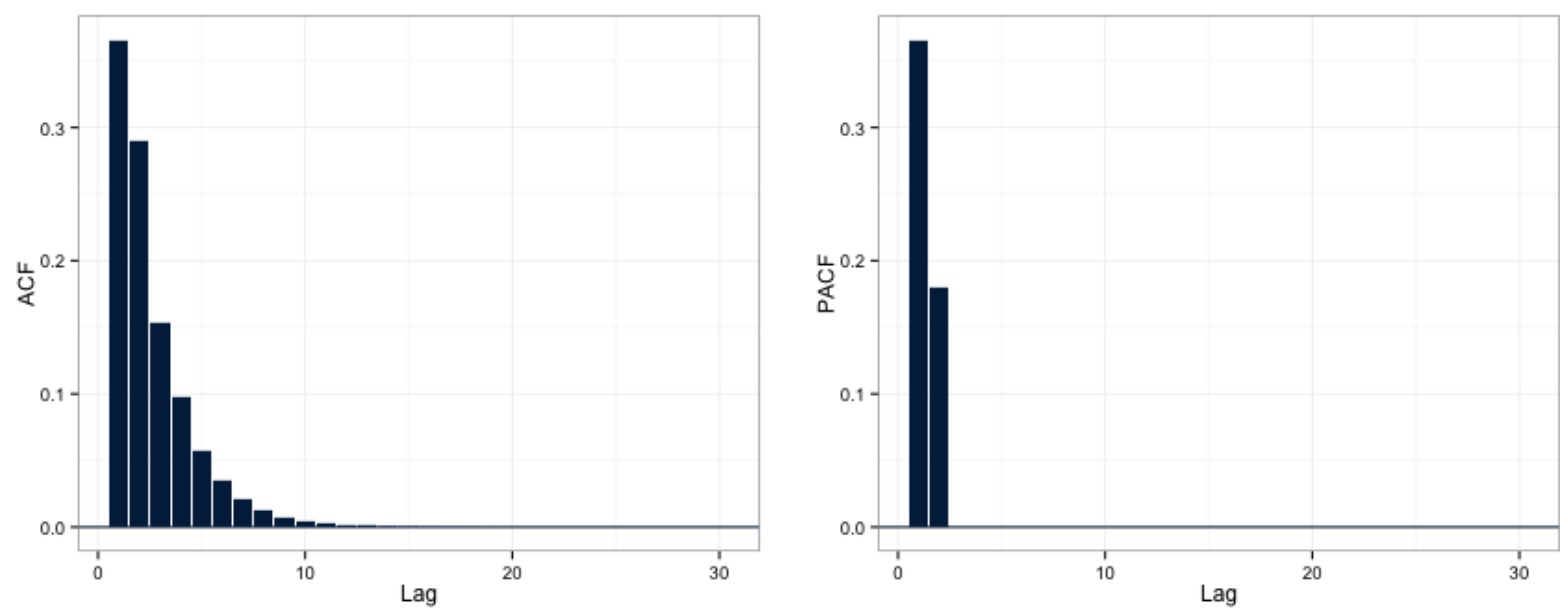

Figura 3.2: Autocorrelação e Autocorrelação parcial teórica: $\operatorname{INAR(2)~com~}\left(\alpha_{1}, \alpha_{2}\right)=(0,3,0,18)$ e $\lambda=3$. 

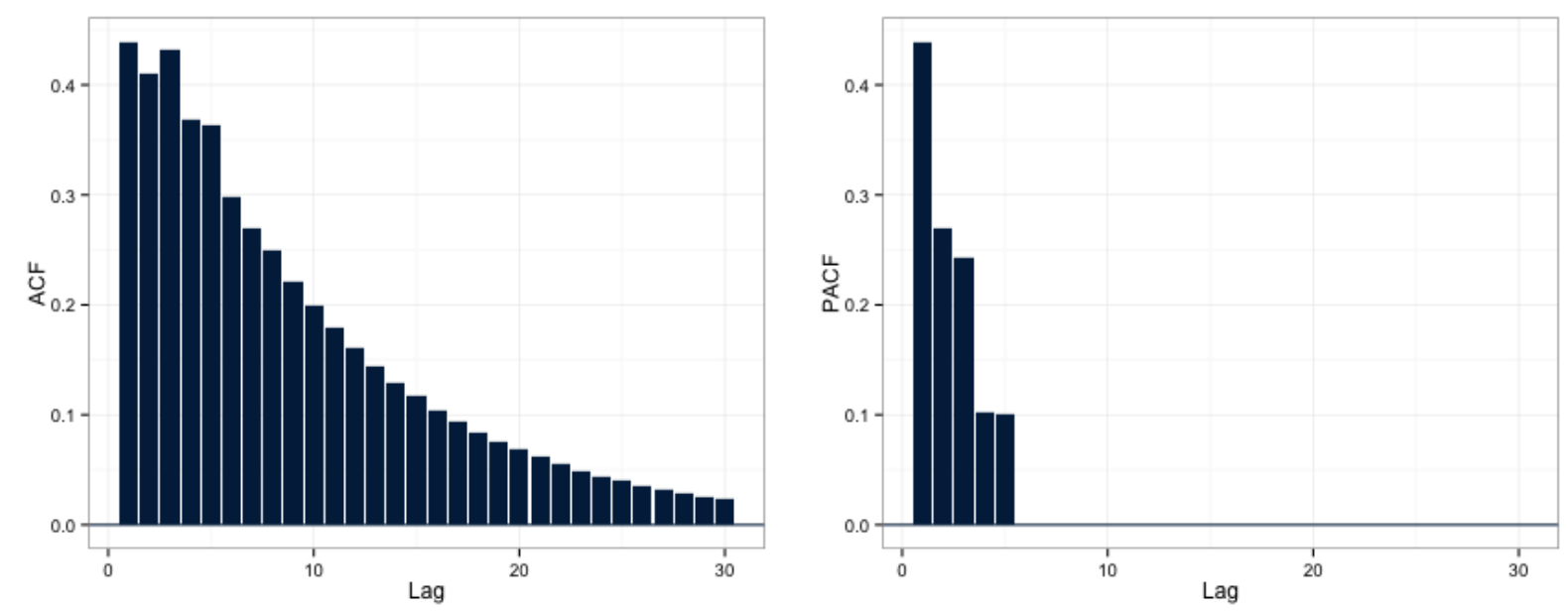

Figura 3.3: Autocorrelação e Autocorrelação parcial teórica: INAR(5) com $\left(\alpha_{1}, \alpha_{2}, \alpha_{3}, \alpha_{4}, \alpha_{5}\right)=$ $(0,22,0,15,0,2,0,08,0,1)$ e $\lambda=1$.

Como visto nas propriedades dos modelos $\operatorname{INAR}(\mathrm{p})$, o comportamento esperado é semelhante ao dos modelos $\mathrm{AR}(\mathrm{p})$ : autocorrelação decaindo exponencialmente e a autocorrelação parcial com valores diferentes de zero até a ordem $p$ e nula nas demais.

\subsubsection{Métodos de estimação}

A estimação dos parâmetros do modelo INAR possui maior complexidade comparada à estimação dos parâmetros do modelo autorregressivo. Isso se dá porque a distribuição de $X_{t}$, condicionada à informação observada até o instante anterior $t-1,\left(X_{t-1}=x_{t-1}, X_{t-2}=x_{t-2}, X_{t-3}=x_{t-3}, \ldots\right)$, é a convolução da distribuição da inovação $I_{t}$ e da soma das binomiais com parâmetros $\left(x_{t-j}, \alpha_{j}\right)$ $j=1,2, \cdots$.

Seguindo o que foi desenvolvido por Al-Osh e Alzaid (1987) para os modelos INAR(1), foram propostos por Jin-Guan e Li-Yuan (1991) e Ruijun Bu e Hadri (2008) os estimadores para o modelo INAR(p); esta sessão mostra os métodos de estimação dos processos INAR com distribuição marginal dada por Poisson.

São apresentados três estimadores:

- Estimador de Yule-Walker

- Estimador de Mínimos Quadrados Condicionais

- Estimador de Máxima Verossimilhança Condicional

O estimador de Yule-Walker (YW) é obtido utilizando as equações de Yule-Walker, que estimam os parâmetros por meio das autocorrelações amostrais. Esse método foi apresentado tanto para o modelo INAR(1), como também para o modelo generalizado, INAR(p).

O método de mínimo quadrados já é bastante conhecido na literatura de modelos de regressão. Sua ideia básica é minimizar o erro esperado dado o modelo proposto. Em séries temporais, pelo fato de existir a dependência linear dos valores passados, utiliza-se o estimador de mínimos quadrados condicionado a observações anteriores, denominado mínimos quadrados condicionais (MQC). Ambos estimadores, YW e MQC, produzem formas analíticas para os estimadores, mesmo se tratando de um problema de minimização no caso do MQC.

O estimador de máxima verossimilhança condicional (MVC) engloba a informação da distribuição marginal do modelo proposto, no caso Poisson. Ele propõe estimativas dos parâmetros tal que a probabilidade, do conjunto de dados observados serem gerados pelo modelo, seja máxima. 


\section{Yule-Walker (YW)}

Os estimadores de Yule-Walker de $\alpha_{j}, j=1,2, \ldots, p$ e $\lambda$ de um modelo $\operatorname{INAR(p),~são~obtidos~}$ de modo equivalente aos estimadores do modelo $\mathrm{AR}(\mathrm{p})$. É possível obter uma expressão analítica para cada $\alpha_{j}$ em função das autocorrelações. Tomando $p=1$ e usando a autocorrelação amostral chega-se a um estimador para $\alpha$ :

$$
\begin{aligned}
\hat{\alpha} & =\frac{\hat{\rho(1)}}{\rho \hat{(0)}} \\
& =\frac{\sum_{t=0}^{n-1}\left(x_{t}-\bar{x}\right)\left(x_{t+1}-\bar{x}\right)}{\sum_{t=0}^{n}\left(x_{t}-\bar{x}\right)^{2}}
\end{aligned}
$$

em que $\bar{x}$ é a média amostral da série $X_{t}$.

É razoável pensar que o estimador de $\lambda$, média da distribuição de Poisson, é dado como a média da sequência $\hat{I}_{t}$, onde $\hat{I}_{t}=x_{t}-\hat{\alpha} x_{t-1}$, para $t=2,3, \cdots, N$,

$$
\hat{\lambda}=\sum_{t=2}^{N} \frac{\hat{I}_{t}}{N-1} .
$$

Generalizando o modelo INAR, considerando o modelo apresentado em (3.3), podemos estimar os parâmetros $\alpha_{j}(j=1, \cdots, p)$ através da estrutura de autocorrelação, da mesma forma que para o modelo INAR(1), utilizando as autocorrelações amostrais. A estrutura recursiva das autocorrelações pode ser expressa em forma de matriz, tendo a expressão

$$
\rho_{k}=\sum_{j=1}^{\min (p, k)} \alpha_{j} \rho_{k-j}
$$

apresentada como

$$
\Gamma \alpha=\rho,
$$

em que

$$
\Gamma=\left[\begin{array}{ccccc}
1 & 0 & 0 & \cdots & 0 \\
\rho_{1} & 1 & 0 & \cdots & 0 \\
\rho_{2} & \rho_{1} & 1 & \cdots & 0 \\
\vdots & \vdots & \vdots & \ddots & \vdots \\
\rho_{p-1} & \rho_{p-2} & \rho_{p-3} & \cdots & 1
\end{array}\right] \quad \alpha=\left[\begin{array}{c}
\alpha_{1} \\
\alpha_{2} \\
\vdots \\
\alpha_{p}
\end{array}\right] \text { e } \quad \rho=\left[\begin{array}{c}
\rho_{1} \\
\rho_{2} \\
\vdots \\
\rho_{p}
\end{array}\right]
$$

Novamente, substituindo $\rho_{j}$ por $\hat{\rho}_{j}$ temos os estimadores de Yule-Walker dados por:

$$
\hat{\Gamma} \hat{\alpha}=\hat{\rho}
$$


Os estimadores de $\lambda$ e $\sigma^{2}$ são, respectivamente,

$$
\begin{aligned}
\hat{\lambda} & =\sum_{i=p+1}^{N} \frac{\hat{I}_{t}}{N-p} \\
e & \\
\hat{\sigma}^{2} & =\frac{1}{N-p} \sum_{t=p+1}^{N}\left(\hat{I}_{t}-\bar{I}\right)^{2},
\end{aligned}
$$

considerando agora $\hat{I}_{t}=x_{t}-\sum_{j=1}^{p} \hat{\alpha_{j}} x_{t-j}$ e $\bar{I}=\frac{1}{N-p} \sum_{t=p+1}^{N} \hat{I}_{t}$

\section{Mínimos Quadrados Condicionais (MQC)}

Como mencionado, o estimador de mínimos quadrados condicionais busca estimadores de $\alpha_{j}$ através da minimização da soma do quadrado dos erros. Seja o modelo dado em (3.3), $F_{t}$ representando toda a informação até o momento $t$, temos

$$
\begin{aligned}
F_{t} & =\mathcal{F}\left(X_{1}, X_{2}, \cdots, X_{t}\right) \\
\beta & =\left(\lambda, \alpha_{1}, \cdots, \alpha_{p}\right) \\
g\left(\beta, F_{t-1}\right) & =\mathbb{E}\left(X_{t} \mid F_{t-1}\right) \\
& =\lambda+\alpha_{1} X_{t-1}+\cdots+\alpha_{p} X_{t-p} .
\end{aligned}
$$

A quantidade que denota a soma do quadrado dos erros é $Q_{N}(\beta)$, dada por

$$
Q_{N}(\beta)=\sum_{t=p+1}^{N}\left\{X_{t}-g\left(\beta, F_{t}\right)\right\}^{2} .
$$

O estimador $\hat{\beta}$ é chamado de mínimos quadrados condicionais porque é obtido minimizando a quantidade dada em (3.11), isto é,

$$
\left.Q_{N}(\hat{\beta})=\min _{\beta}\left\{Q_{N}(\beta)\right)\right\}
$$

Essa estimativa é obtida através das derivadas parciais em relação a cada componente de $\beta$ igualada a zero. A forma explícita dos estimadores de MQC é dada por:

$$
\hat{\lambda}=\bar{X}_{N}^{(0)}-\sum_{j=1}^{p} \hat{\alpha}_{j} \bar{X}_{N}^{(j)}
$$

$$
\hat{\Gamma} \hat{\alpha}=\hat{\rho},
$$


em que

$$
\begin{gathered}
\bar{X}_{N}^{(j)}=\frac{1}{N-p} \sum_{t=p+1}^{N} X_{t-j}, \\
\hat{\gamma}_{k-j}=\frac{1}{N-p} \sum_{t=p+1}^{N}\left(X_{t-j}-\bar{X}_{N}^{(j)}\right)\left(X_{t-1}-\bar{X}_{N}^{(k)}\right), \\
\hat{\rho}_{k-j}=\frac{\hat{\gamma}_{k-j}}{\hat{\gamma}_{0}}, \\
\hat{\Gamma}=\left[\begin{array}{ccccc}
1 & 0 & 0 & \cdots & 0 \\
\hat{\rho}_{1} & 1 & 0 & \cdots & 0 \\
\hat{\rho}_{2} & \hat{\rho}_{1} & 1 & \cdots & 0 \\
\vdots & \vdots & \vdots & \ddots & \vdots \\
\hat{\rho}_{p-1} & \hat{\rho}_{p-2} & \hat{\rho}_{p-3} & \cdots & 1
\end{array}\right] .
\end{gathered}
$$

É fácil notar que a medida que o tamanho da série $N$ aumenta, os estimadores de mínimos quadrados condicionais e de Yule-Walker tornam-se equivalentes.

\section{Máxima Verossimilhança Condicional (MVC)}

Por fim, a metodologia de estimação por máxima verossimilhança condicional (MVC), muito utilizada em modelos de séries temporais, leva em conta a dependência temporal entre os registros ordenados no tempo. O estimador de máxima verossimilhança possui boas propriedades, ele busca a estimativa para os parâmetros do modelo baseando-se na distribuição conjunta do processo $\left\{X_{t}\right\}$. Para estimar os parâmetros do modelo busca-se o conjunto de parâmetros que maximiza essa probabilidade. O modelo INAR(p), definido em (3.3), tem a verossimilhança dada pela convolução das $p$ binomiais com a Poisson. Considera-se a probabilidade conjunta, condicionada aos $p$ primeiros valores, como:

$$
L\left(\alpha_{1}, \alpha_{2}, \cdots, \alpha_{p}, \lambda\right)=\prod_{t=p+1}^{N} P\left(X_{t} \mid X_{t-1}, \cdots, X_{t-p}\right) P\left(X_{t-1}, \cdots, X_{t-p}\right)
$$

em que

$$
\begin{aligned}
& P\left(X_{t} \mid X_{t-1}, \cdots, X_{t-p}\right)=\quad \sum_{i_{1}=0}^{\min \left(X_{t-1}, X_{t}\right)} \quad\left(\begin{array}{c}
X_{t-1} \\
i_{1}
\end{array}\right) \alpha_{1}^{i_{1}}\left(1-\alpha_{1}\right)^{\left(X_{t-1}-i_{1}\right)} \times \\
& \sum_{i_{2}=0}^{\min \left(X_{t-2}, X_{t}-i_{1}\right)} \quad\left(\begin{array}{c}
X_{t-2} \\
i_{2}
\end{array}\right) \alpha_{2}^{i_{2}}\left(1-\alpha_{2}\right)^{\left(X_{t-2}-i_{2}\right)} \times
\end{aligned}
$$

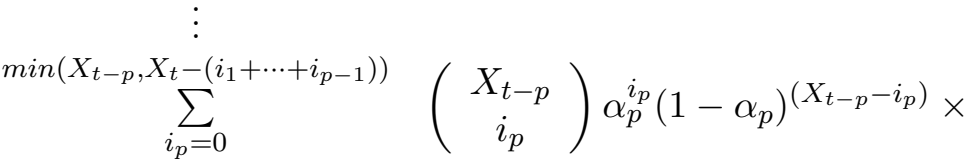

$$
\begin{aligned}
& \frac{e^{-\lambda} \lambda^{X}-\left(i_{1}+\cdots+i_{p}\right)}{\left[X_{t}-\left(i_{1}+\cdots+i_{p}\right)\right] !}
\end{aligned}
$$


A estimativa de MVC para o vetor de parâmetros $\left(\alpha_{1}, \alpha_{2}, \cdots, \alpha_{p}, \lambda\right)^{\prime}$ é obtida através da maximização de L dado em (3.19) com a probabilidade dada por (3.20). Ruijun Bu e Hadri (2008) realizaram um estudo de eficiência assintótica relativa dos estimadores de MVC em comparação com os estimadores MQC e confirmaram que os estimadores de MVC são assintoticamente mais eficientes.

\subsubsection{Estudo de simulação}

O estudo de simulação busca avaliar e comparar os métodos de estimação em alguns processos pré definidos, testando a proximidade da estimativa com o parâmetro real usado para gerar a série. Primeiro realiza-se o estudo de simulação utilizando séries simuladas através de um modelo INAR de ordem 1; em seguida o modelo INAR de ordem 2 tem os resultados expostos.

Os modelos gerados possuem a estrutura apresentada em (3.3) em que a parte dada pela inovação segue uma distribuição Poisson com $\lambda=1$. Já o parâmetro da probabilidade associado ao operador thinning binomial pertence ao conjunto $(0,3 ; 0,5 ; 0,7)$. Cada modelo de ordem 1 foi gerado com 3 tamanhos de séries distintos, $\mathrm{N}=100,200$ e 500 enquanto que os modelos INAR de odem 2 tiveram um único tamanho gerado, $\mathrm{N}=200$. Ao final 500 réplicas foram geradas de cada modelo apresentado na Tabela 3.2.

\begin{tabular}{lccc}
\hline \hline \multicolumn{3}{c}{ Modelos gerados com $\lambda=1$} \\
\hline Modelo & $\mathrm{N}$ & $\alpha_{1}$ & $\alpha_{2}$ \\
\hline \hline $\operatorname{INAR}(1)$ & 100 & 0,3 & - \\
$\operatorname{INAR}(1)$ & 100 & 0,5 & - \\
$\operatorname{INAR}(1)$ & 100 & 0,7 & - \\
\hline $\operatorname{INAR}(1)$ & 200 & 0,3 & - \\
$\operatorname{INAR}(1)$ & 200 & 0,5 & - \\
$\operatorname{INAR}(1)$ & 200 & 0,7 & - \\
\hline $\operatorname{INAR}(1)$ & 500 & 0,3 & - \\
$\operatorname{INAR}(1)$ & 500 & 0,5 & - \\
$\operatorname{INAR}(1)$ & 500 & 0,7 & - \\
\hline $\operatorname{INAR}(2)$ & 200 & 0,3 & 0,3 \\
$\operatorname{INAR}(2)$ & 200 & 0,5 & 0,3 \\
\hline $\operatorname{INAR}(2)$ & 200 & 0,3 & 0,5 \\
\hline \hline
\end{tabular}

Tabela 3.2: Modelos gerados - INAR.

O estudo de simulação segue a sequência:

- Gera-se uma trajetória de um modelo definido.

- Estima-se os parâmetros utilizando os estimadores dado na Seção 3.1.3.

- Armazena-se as estimativas de $\alpha_{j}$ e $\lambda$.

Cada etapa é repetida 500 vezes e os valores são salvos.

Cada modelo tem uma trajetória mostrada como exemplo, além da sua distribuição incondicional, autocorrelação e autocorrelação parcial. Em seguida realiza-se uma análise das estimativas, viés e EQM. Ao final do estudo mostram-se tabelas resumo de cada processo. Os resultados dos modelos INAR(1), com $\lambda=1$, são apresentados nas Tabelas 3.3 e 3.4. Os modelos gerados por um processo INAR(2) tem os resultados mostrados nas Tabelas 3.6 e 3.7 . 
Processo INAR(1): $X_{t}=0,3 \circ X_{t-1}$

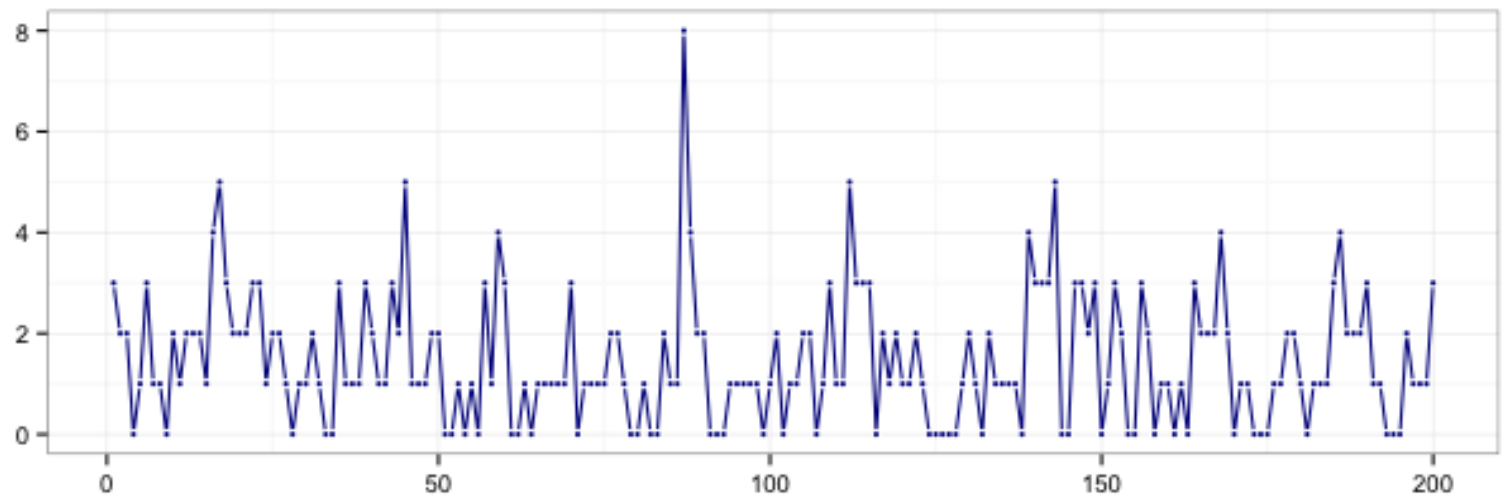

Figura 3.4: Gráfico de uma série simulada, $\operatorname{INAR(1):~} \alpha=0,3$ e $\lambda=1$.

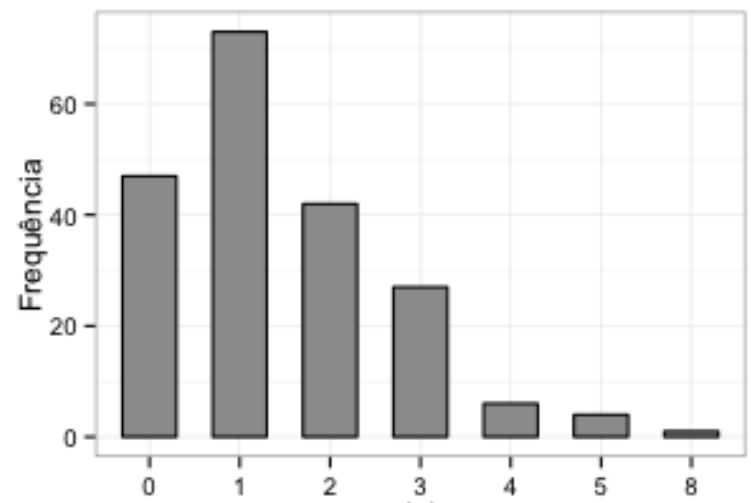

(a)

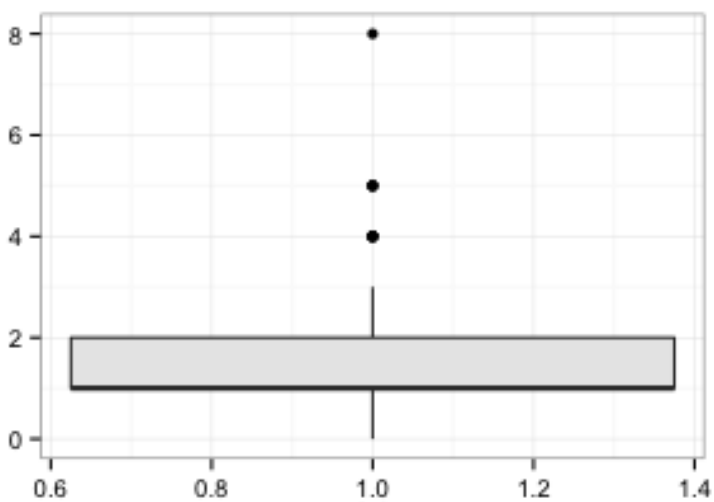

(b)

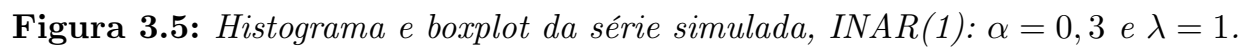
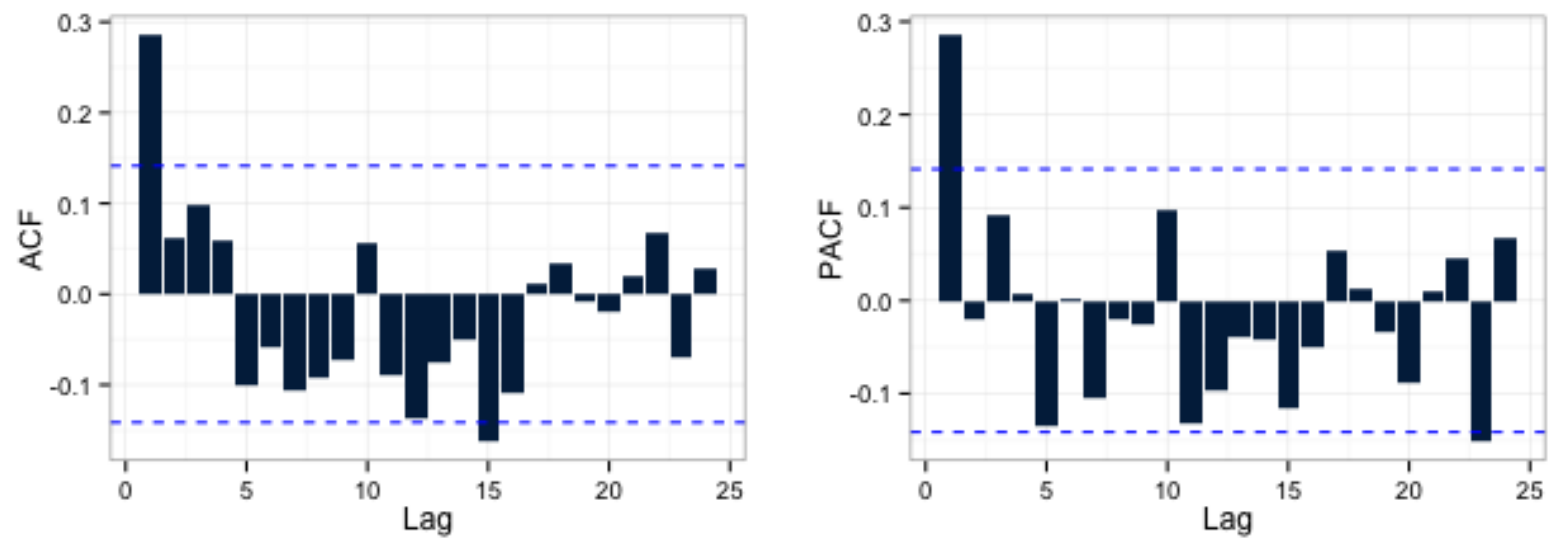

Figura 3.6: Autocorrelação e Autocorrelação parcial amostrais $\operatorname{INAR(1):~} \alpha=0,3$ e $\lambda=1$.

A Figura 3.4 mostra uma trajetória de uma série gerada pelo modelo $\operatorname{INAR}(1), X_{t}=0,3 \circ$ $X_{t-1}+I_{t}$ em que $I_{t}$ possui distribuição Poisson(1). A Figura 3.5 mostra o histograma (a) e boxplot (b) da série, é fácil notar que os valores estão concentrados entre 1 e 2 , próximos à esperança do processo, $\frac{1}{1-0,3}=1,43$. O ACF e PACF da série são mostrados na Figura 3.6. 
O primeiro processo analisado segue um processo $X_{t}=0,3 \circ X_{t-1}$ com inovação dada por um processo de Poisson(1). Os métodos de estimação utilizados são os de Yule-Walker (YW), máxima verossimilhança condicional (MVC) e mínimos quadrados condicional (MQC).

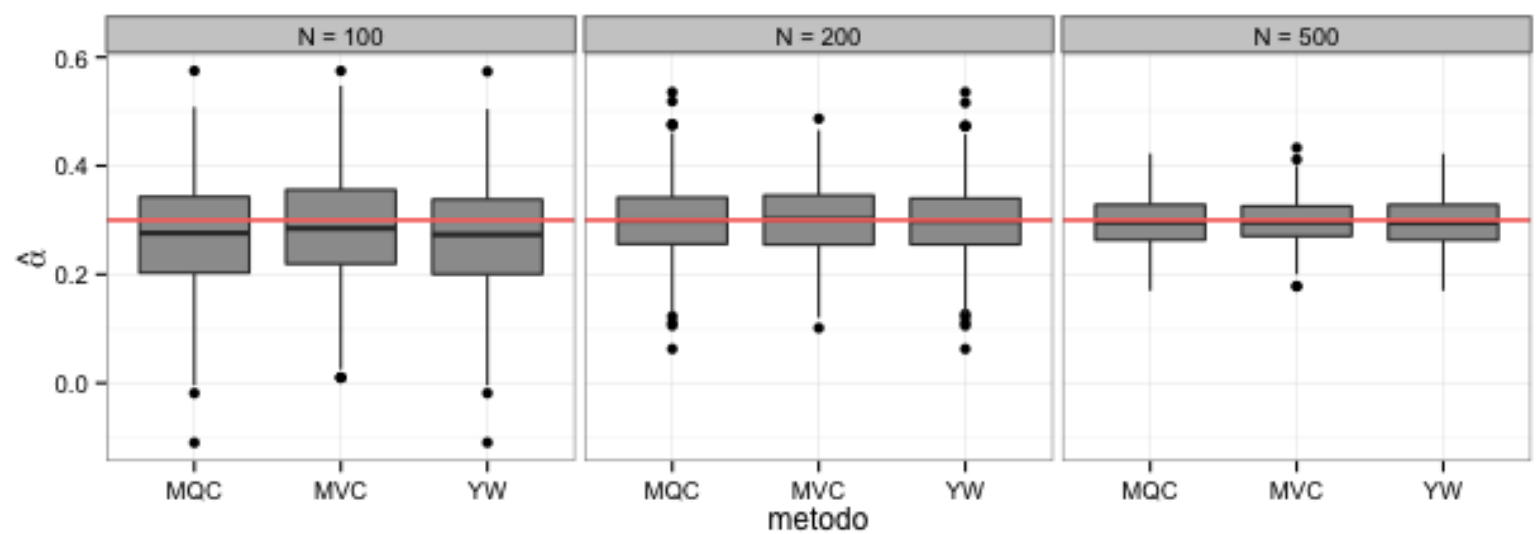

Figura 3.7: Boxplot das estimativas de $\alpha$ para o modelo gerado por $X_{t}=0,3 \circ X_{t-1}+I_{t}$.

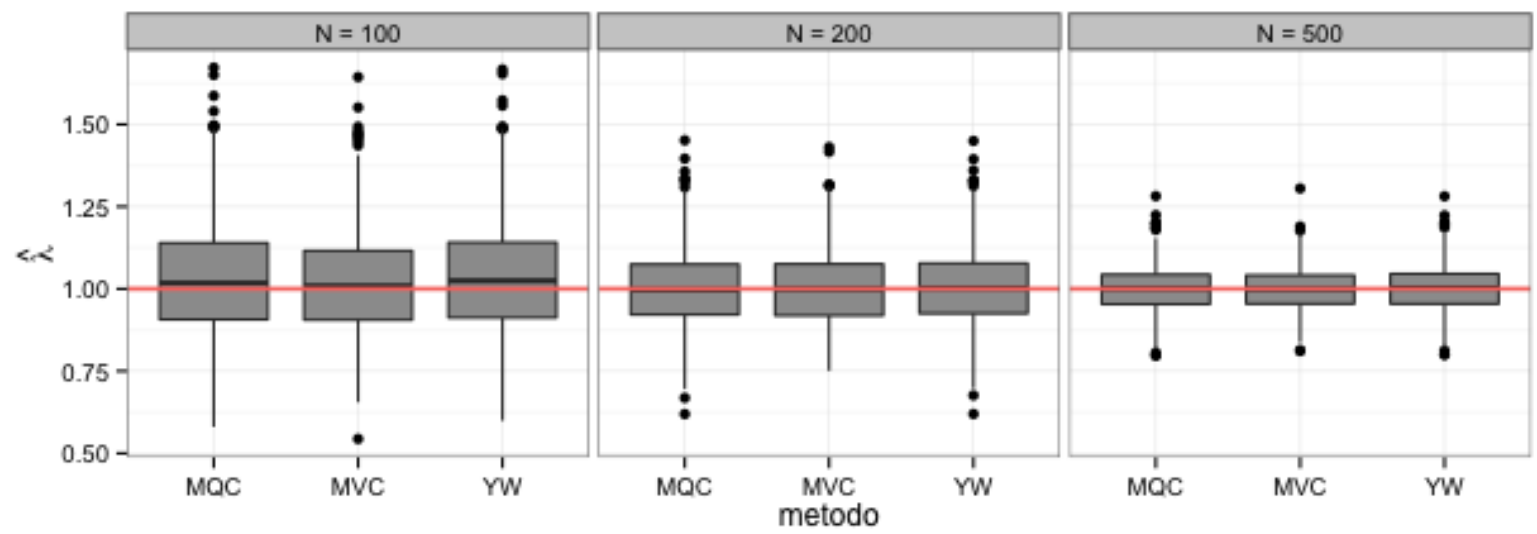

Figura 3.8: Boxplot das estimativas de $\lambda$ para o modelo gerado por $X_{t}=0,3 \circ X_{t-1}+I_{t}$.

A Figura 3.7 apresenta o boxplot das estimativas das 500 réplicas para cada tamanho da série gerada e cada metodologia de estimação. A linha vermelha denota o verdadeiro valor do parâmetro, $\alpha=0,3$. Nota-se que o método de Máxima Verossimilhança Condicional está sempre mais próximo ao verdadeiro valor. Como esperado, a medida que o tamanho da série aumenta, a variância também reduz e as estimativas se concentram mais em torno de 0,3 . A distribuição das estimativas de $\lambda$ são mostradas na Figura 3.8, as características da distribuição se repetem com a redução da variabilidade a medida que o tamanho da série aumenta e novamente as estimativas dadas por MVC são mais próximas do parâmetro $\lambda$. 
As Figruas 3.9 e 3.10 mostram os histogramas das estimativas das simulações. Quando falamos em histograma alisado, trata se da união dos valores médios de cada classe.

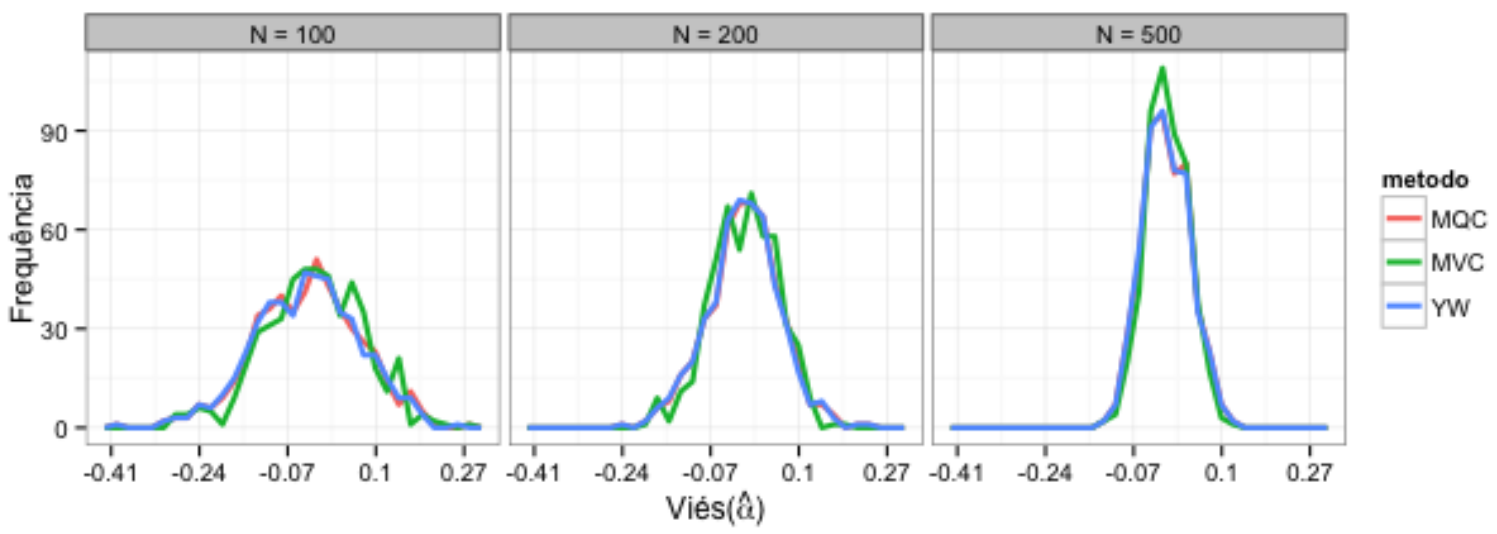

Figura 3.9: Histograma alisado do viés de $\hat{\alpha}$ para o modelo gerado por $X_{t}=0,3 \circ X_{t-1}+I_{t}$.

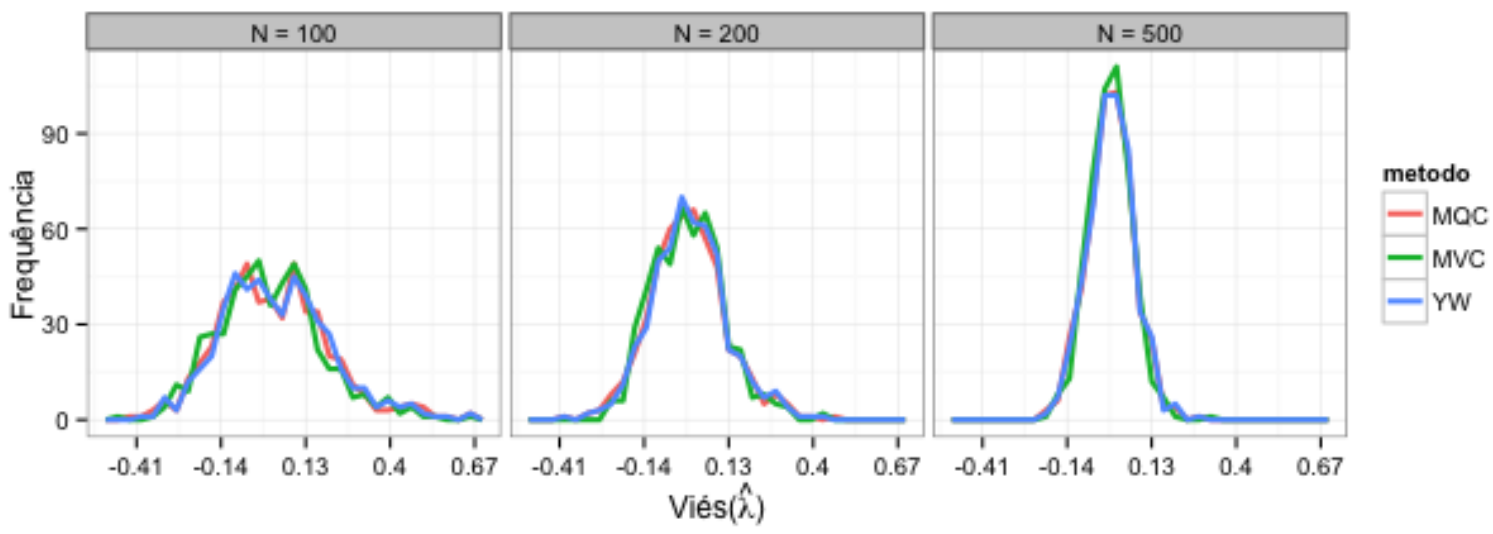

Figura 3.10: Histograma alisado do viés de $\hat{\lambda}$ para o modelo gerado por $X_{t}=0,3 \circ X_{t-1}+I_{t}$.

A Figura 3.9 mostra a curva do viés de $\hat{\alpha}$ e a Figura 3.10 o viés de $\hat{\lambda}$, para cada método de estimação em diferentes tamanhos de séries. Os três métodos são bastante próximos sob essa ótica, a medida que cresce o tamanho da série, o viés tende a ser menor e mais concentrado em torno de zero. 
Processo INAR(1): $X_{t}=0,5 \circ X_{t-1}$

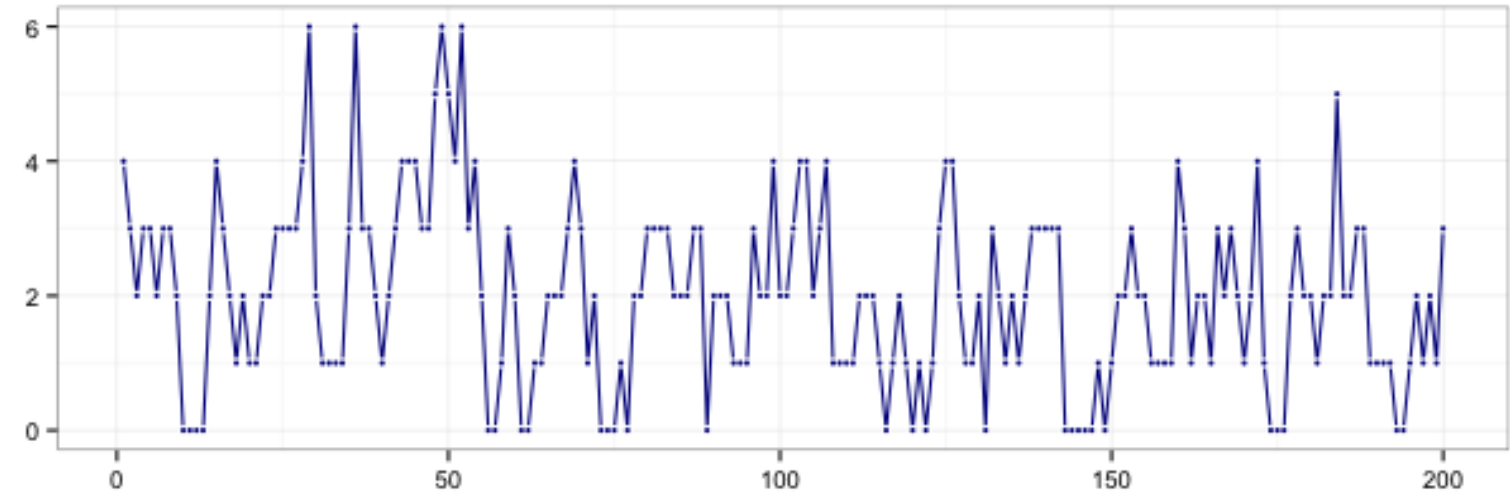

Figura 3.11: Gráfico de uma série simulada, $\operatorname{INAR(1):~} \alpha=0,5$ e $\lambda=1$.

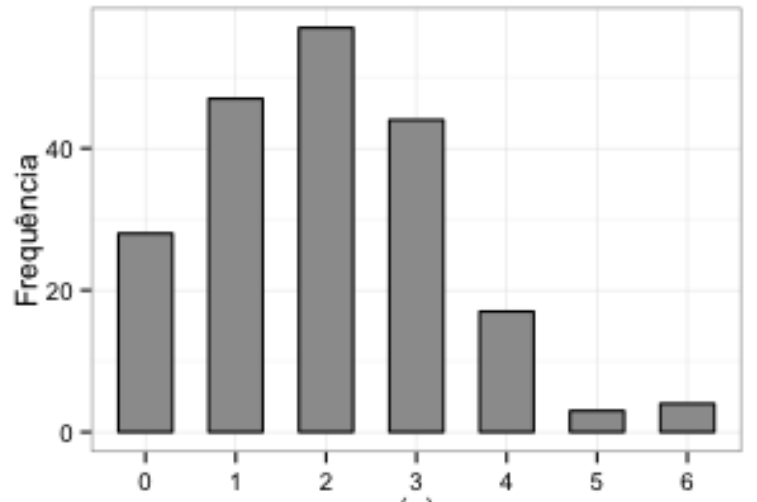

(a)

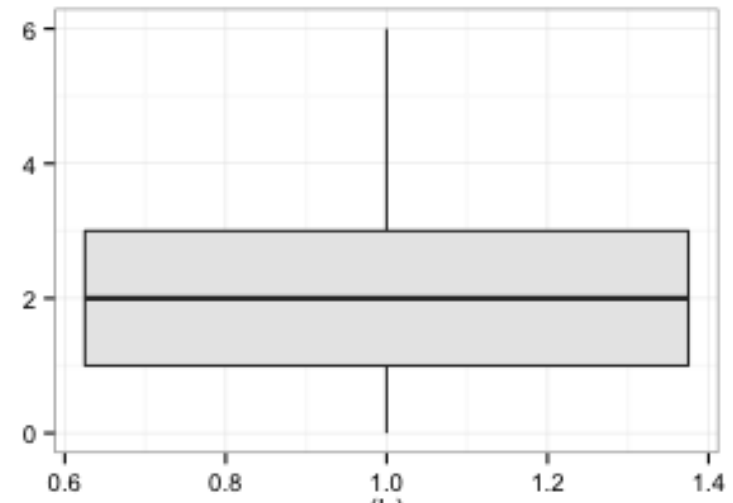

(b)

Figura 3.12: Histograma e boxplot da série simulada, INAR(1): $\alpha=0,5$ e $\lambda=1$.
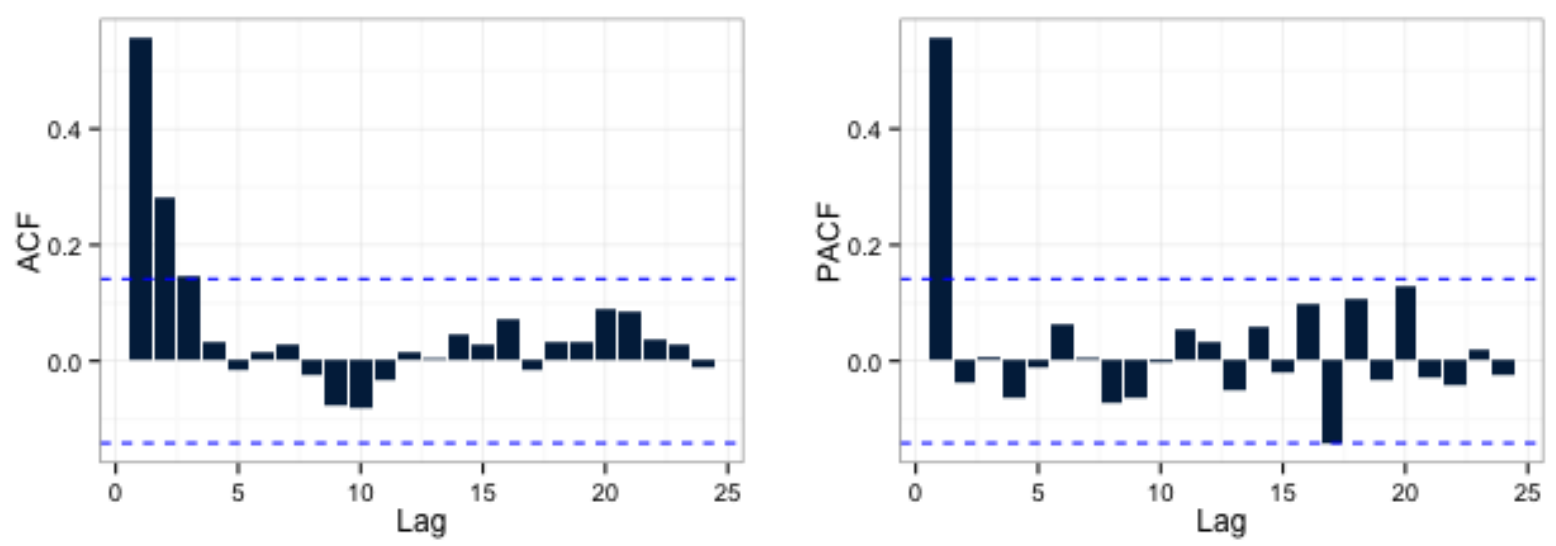

Figura 3.13: Autocorrelação e Autocorrelação parcial amostrais INAR(1): $\alpha=0,5$ e $\lambda=1$.

Outra trajetória é exemplificada na Figura 3.11, gerada pelo modelo INAR(1), $X_{t}=0,5 \circ X_{t-1}+$ $I_{t}$ com a inovação $I_{t}$ dada pela Poisson(1). Nota-se pelas Figuras 3.12 (a) e (b) que o valor médio da série está próximo de $\frac{1}{1-0,5}=2$, valor esperado do processo. O ACF e PACF mostrado na Figura 3.13 apresenta o comportamento esperado mostrado na Figura 3.1, com decaimento exponencial na ACF e lag significante de ordem 1 na PACF. 
A estimação dos parâmetros do processo INAR gerado por $X_{t}=0,5 \circ X_{t-1}$, tem os resultados apresentados nas Figuras 3.14 e 3.15.

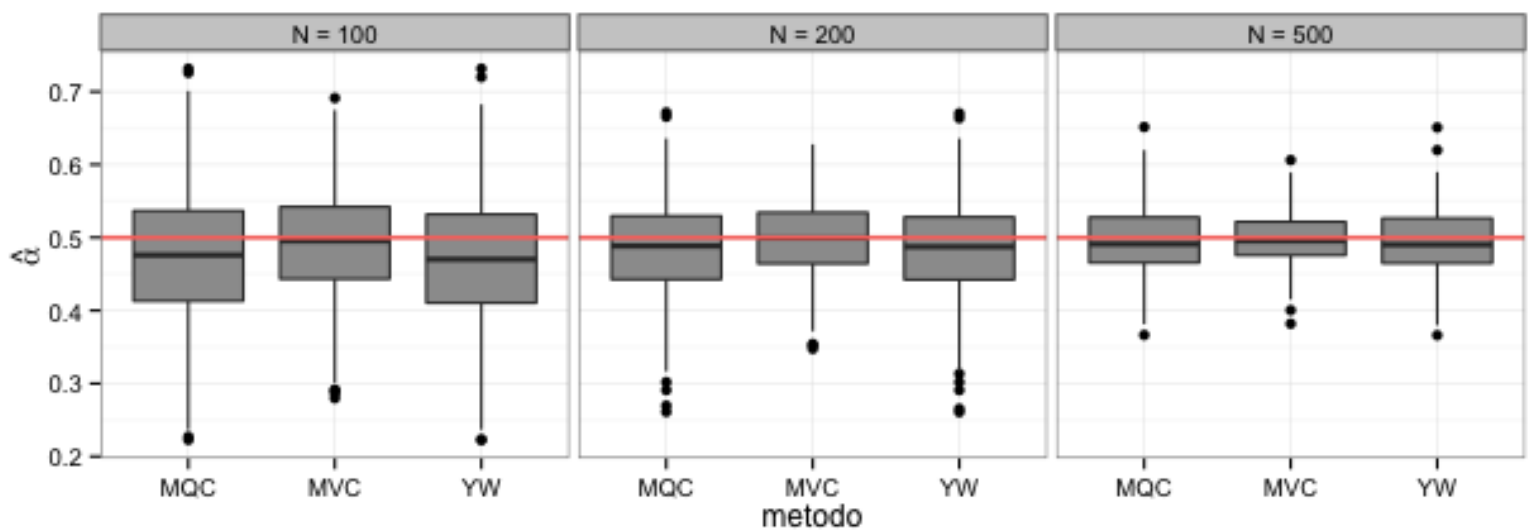

Figura 3.14: Boxplot das estimativas de $\alpha$ para o modelo gerado por $X_{t}=0,5 \circ X_{t-1}+I_{t}$.

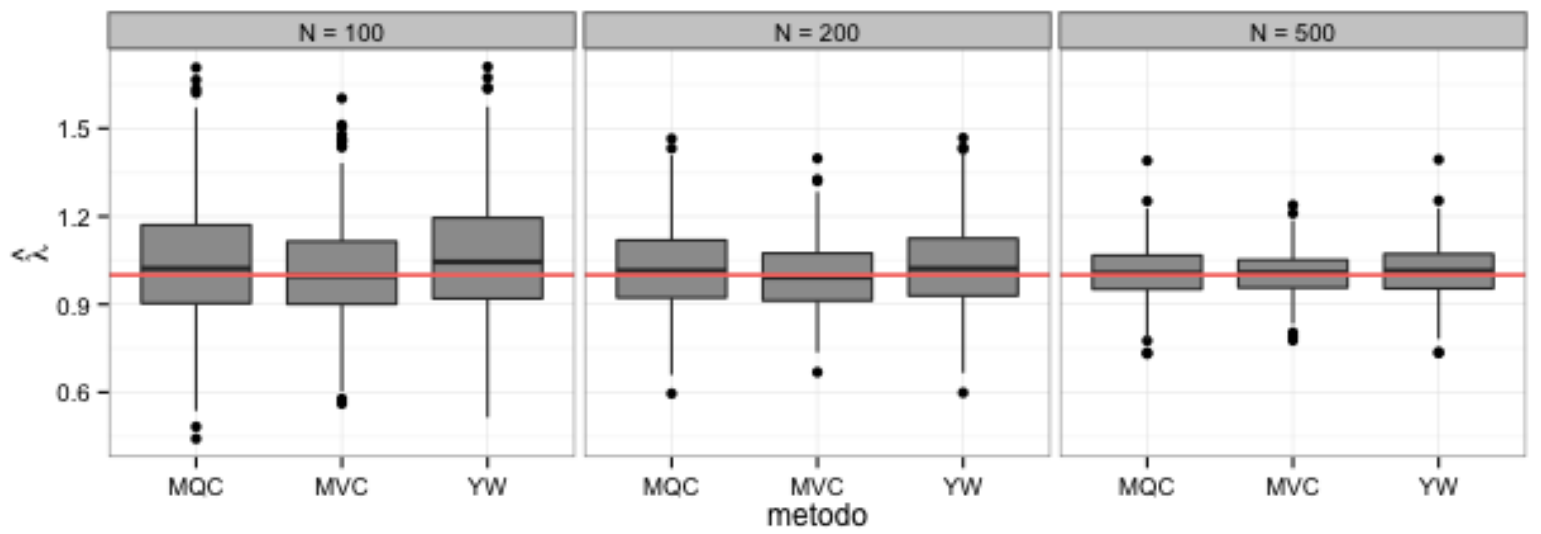

Figura 3.15: Boxplot das estimativas de $\lambda$ para o modelo gerado por $X_{t}=0,5 \circ X_{t-1}+I_{t}$.

Os resultados para o modelo com $\alpha=0,5$ já apresentam uma outra característica do estimador de máxima verossimilhança, que não era tão evidente $\operatorname{com} \alpha=0,3$. A variabilidade dos valores estimados por MVC é menor que a dos outros métodos, isto se torna claro a medida que tomamos um $\alpha$ maior. O mesmo se ve em relação à estimativa de $\lambda$, as estimativas estão mais concentradas cerca do verdadeiro valor quando estima-se com MVC. Essas afirmações são comprovadas nos gráficos boxplot, Figura 3.14 e 3.15 e também no quadro de variâncias na Tabela 3.5. 

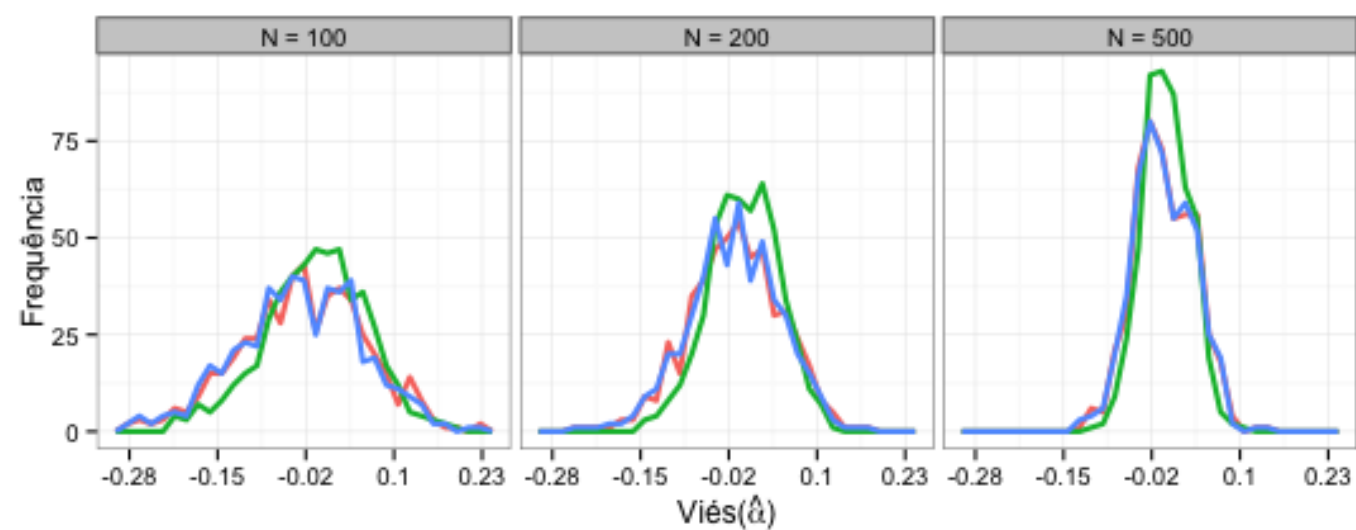

metodo

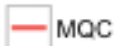

- MvC

$-\mathrm{rW}$

Figura 3.16: Histograma alisado do viés de $\hat{\alpha}$ para o modelo gerado por $X_{t}=0,5 \circ X_{t-1}+I_{t}$.
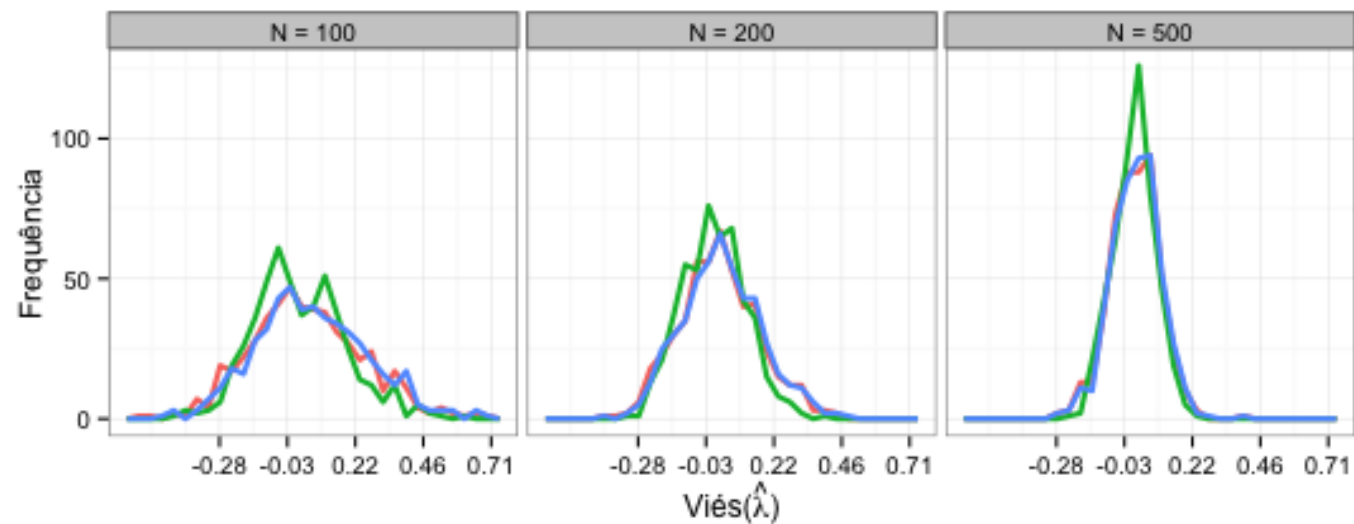

metodo

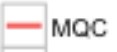

- Mvc

$-\mathrm{Yw}$

Figura 3.17: Histograma alisado do viés de $\hat{\lambda}$ para o modelo gerado por $X_{t}=0,5 \circ X_{t-1}+I_{t}$.

As Figuras 3.16 e 3.17 mostram a estimativa da distribuição do viés no caso do modelo dado por $X_{t}=0,5 \circ X_{t-1}$. A curva do estimador de MVC distoa das demais em todos os tamanhos das séries, isso porque a estimativa de MVC é mais concentrada no verdadeiro valor, fazendo o viés mais concentrado em zero. Com o valor de $\alpha=0,5$ tem-se uma ideia de simetria das curvas em torno de zero. A medida que aumentamos o $\alpha$, nota-se que essa simetria não se mantém. 
Processo INAR(1): $X_{t}=0,7 \circ X_{t-1}$

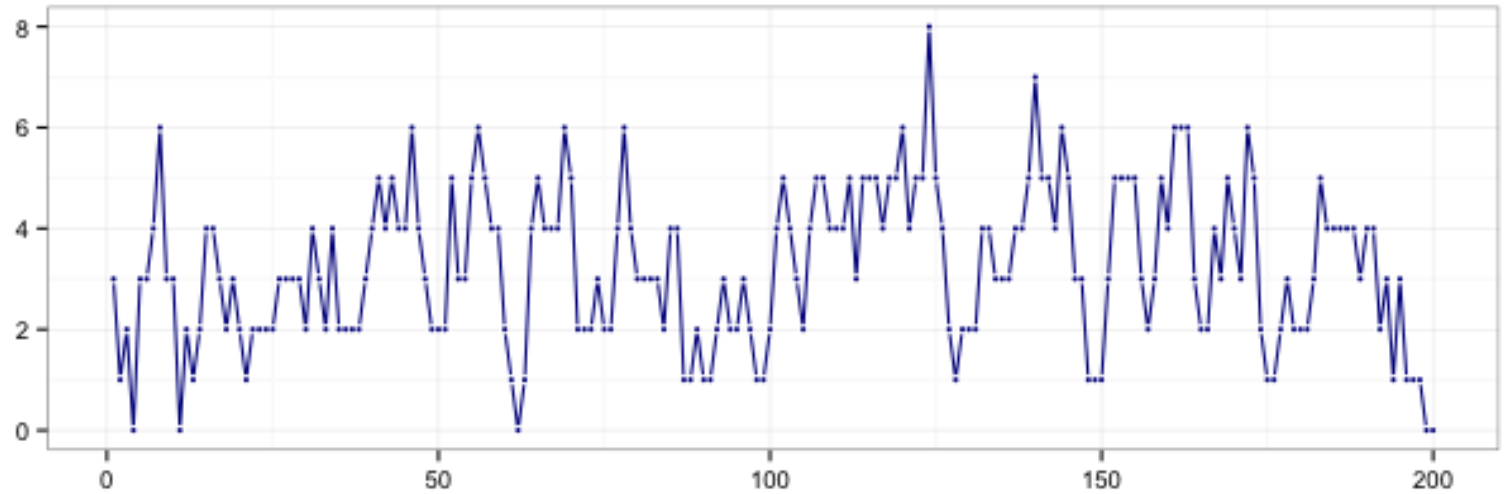

Figura 3.18: Gráfico de uma série simulada, $\operatorname{INAR(1):~} \alpha=0,7$ e $\lambda=1$.

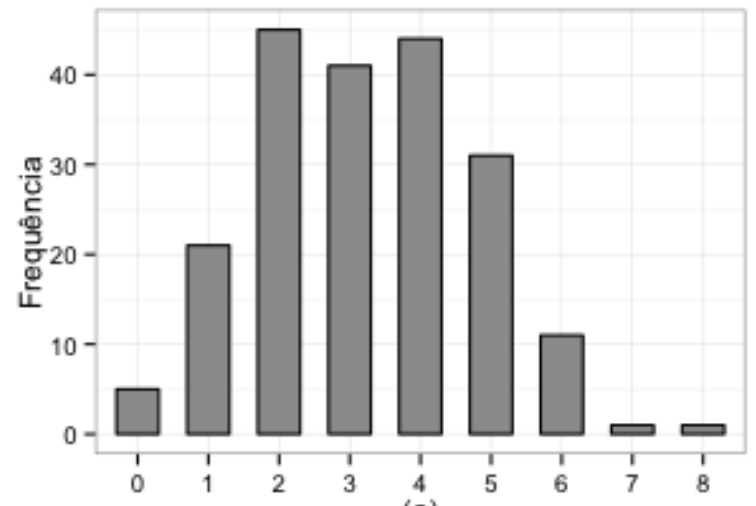

(a)

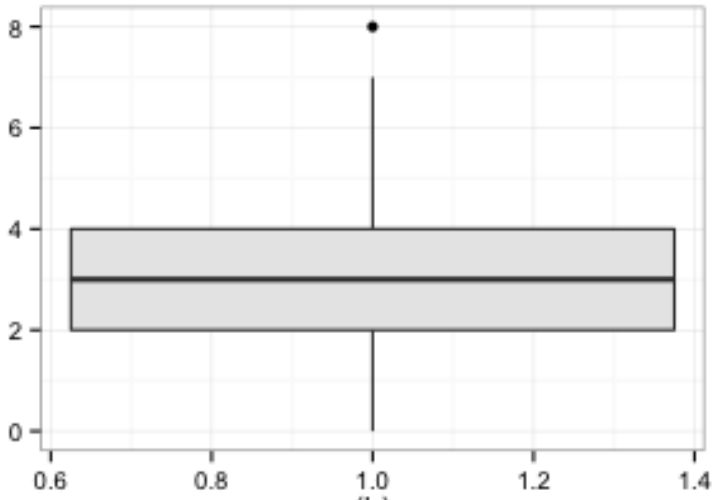

(b)

Figura 3.19: Histograma e boxplot da série simulada, $\operatorname{INAR(1):~} \alpha=0,7$ e $\lambda=1$.
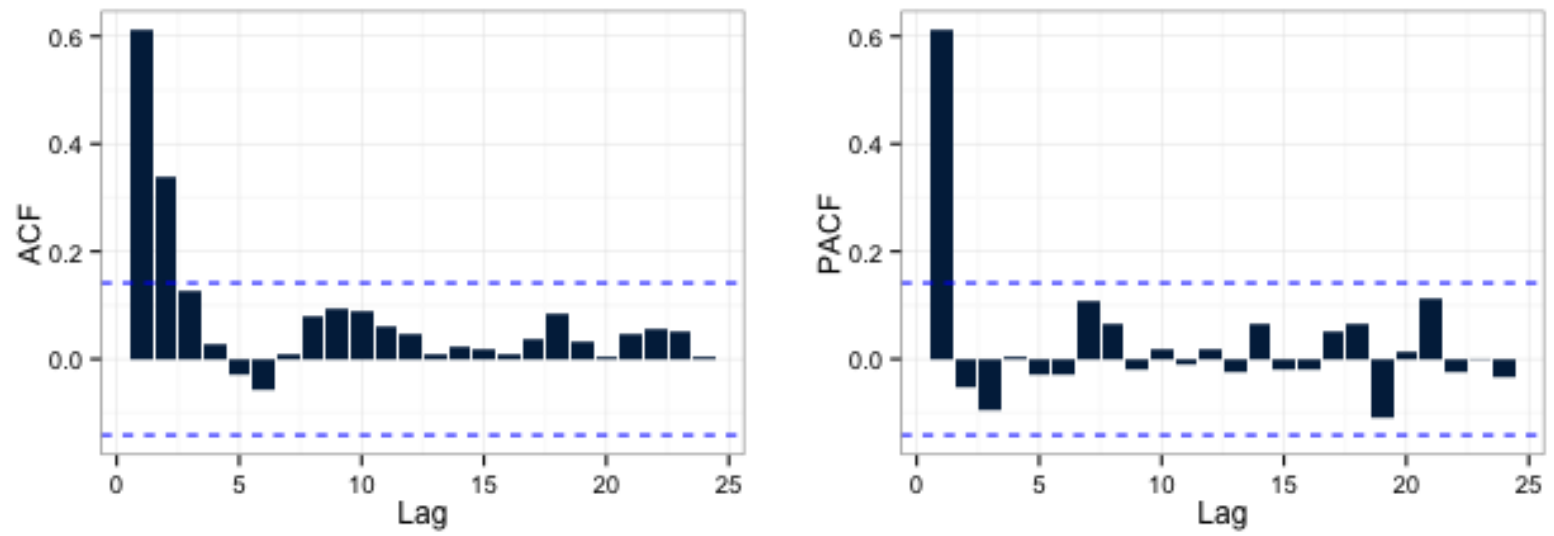

Figura 3.20: Autocorrelação e Autocorrelação parcial amostrais $\operatorname{INAR(1):~} \alpha=0,7$ e $\lambda=1$.

Novamente o primeiro gráfico apresentado na Figura 3.18 mostra a trajetória, agora de um modelo gerado pelo processo INAR $X_{t}=0,7 \circ X_{t-1}+I_{t}$ com $I_{t}$ dada pela Poisson(1). Conseguimos ver nas Figuras 3.19 (a) e (b) que a média da série simulada é muito próxima da esperança do processo, dada por $\frac{1}{1-0,7}=3,33$. O ACF e PACF mostram um comportamento adequado ao modelo gerado. 
As estimações dos parâmetros do processo $X_{t}=0,7 \circ X_{t-1}$ tem os resultados apresentados.

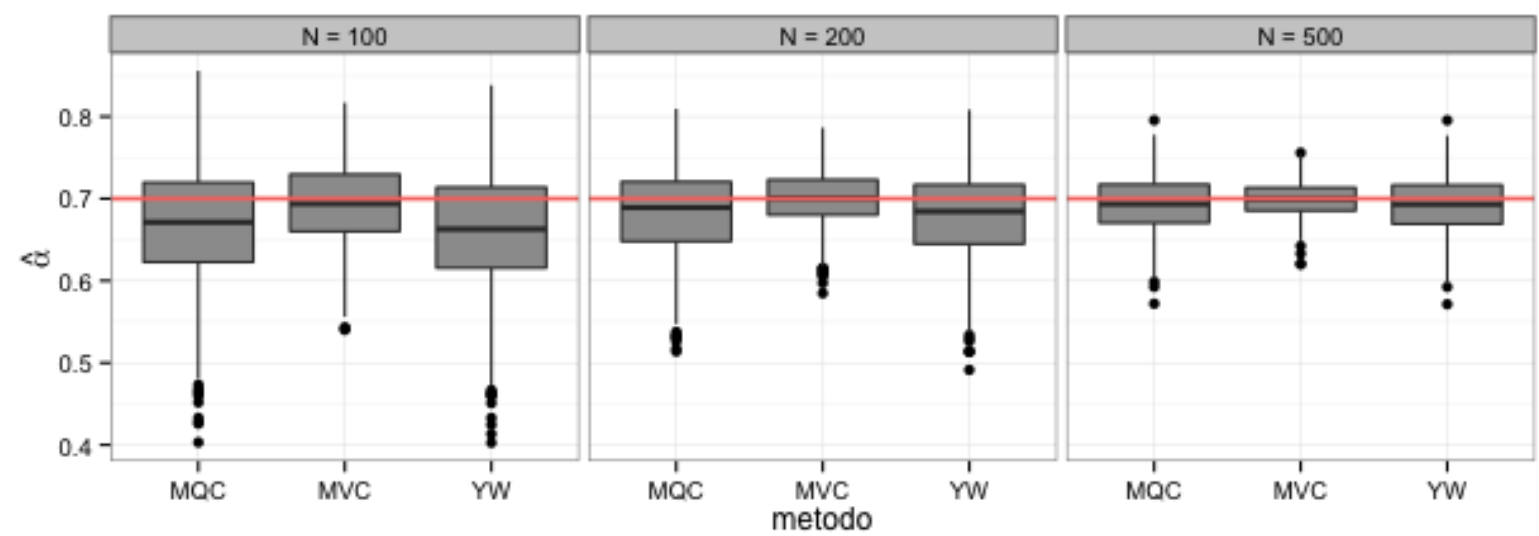

Figura 3.21: Boxplot das estimativas de $\alpha$ para o modelo gerado por $X_{t}=0,7 \circ X_{t-1}+I_{t}$.

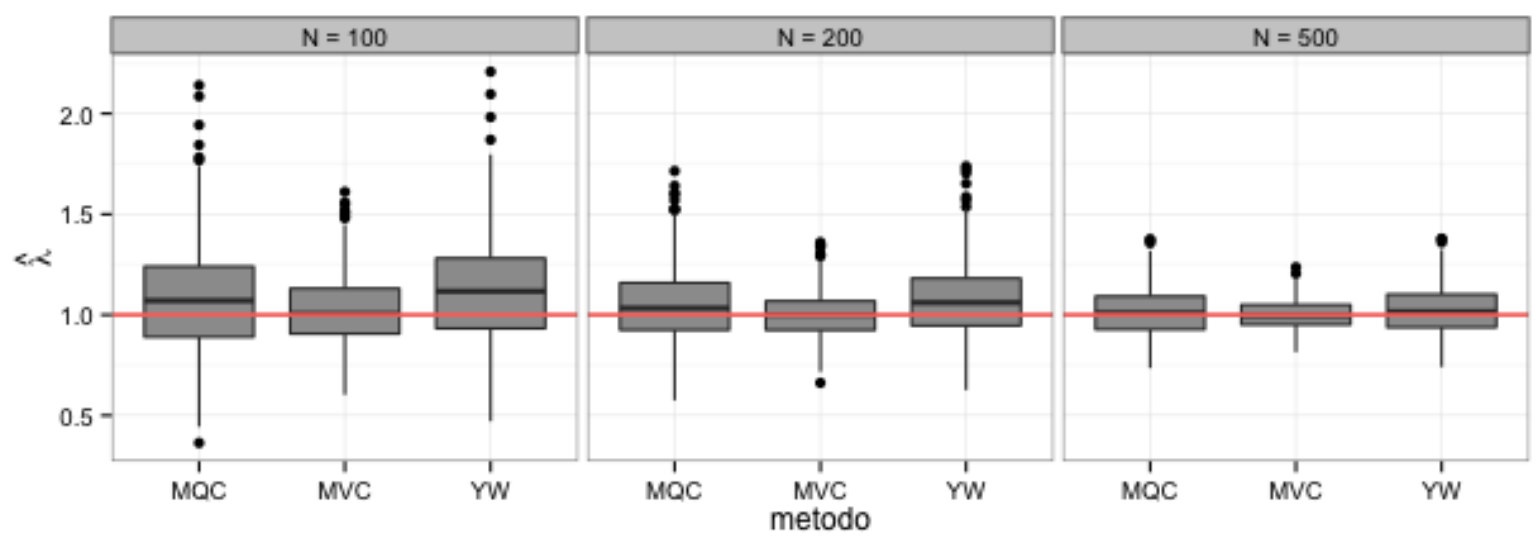

Figura 3.22: Boxplot das estimativas de $\lambda$ para o modelo gerado por $X_{t}=0,7 \circ X_{t-1}+I_{t}$.

A análise das Figuras 3.21 e 3.22 mostra a evidência de que a variabilidade das estimativas obtidas por máxima verossimilhança condicional é menor. Além dessa constatação, nota-se que mesmo para séries com menor tamanho $(\mathrm{N}=100)$ o estimador de $\mathrm{MVC}$ permanece bastante próximo do verdadeiro valor, enquanto os métodos de MQC e YW tendem a subestimar o parâmetro. Para a estimativa de $\lambda$, o estimador de MVC também é mais assertivo e com menor variabilidade; os estimadores de YW e MQC superestimam nesse caso. 

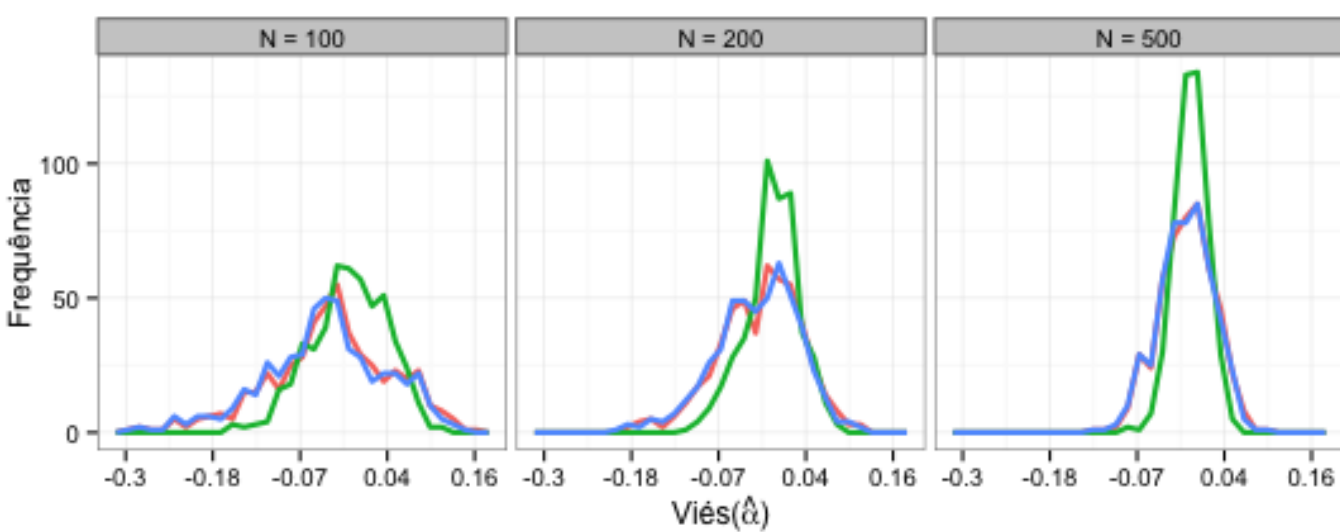

metodo

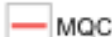

- MVC

$-\mathrm{YW}$

Figura 3.23: Histograma alisado do viés de $\hat{\alpha}$ para o modelo gerado por $X_{t}=0,7 \circ X_{t-1}+I_{t}$.
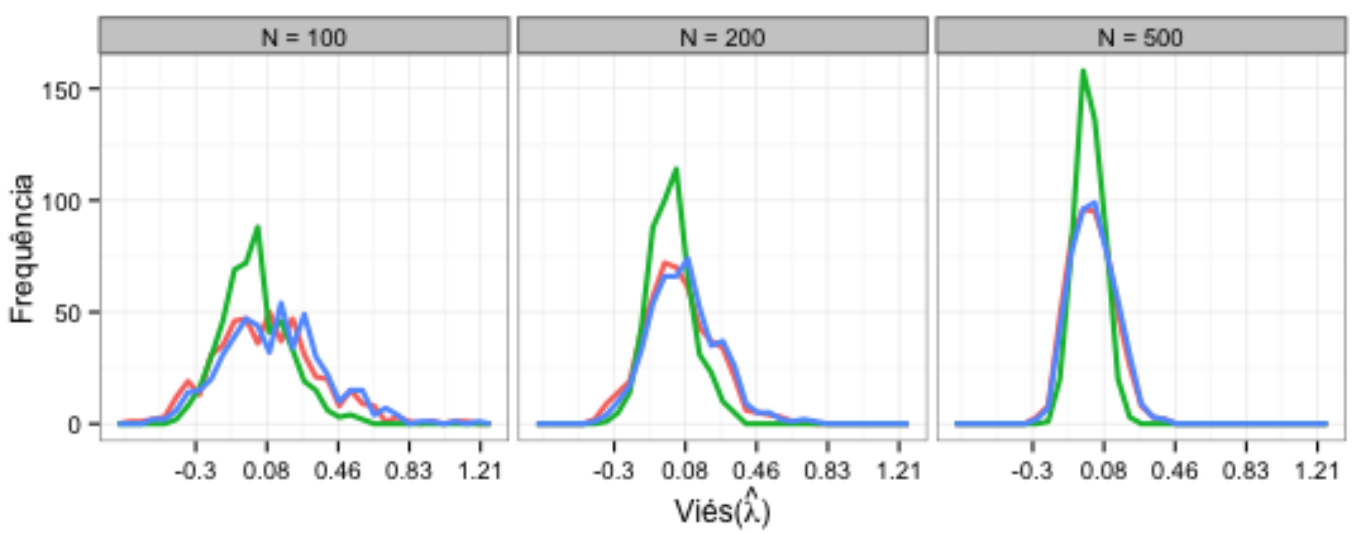

metodo

- MQC

- MvC

$-\mathrm{rw}$

Figura 3.24: Histograma alisado do viés de $\hat{\lambda}$ para o modelo gerado por $X_{t}=0,7 \circ X_{t-1}+I_{t}$.

As curvas apresentadas nas Figuras 3.23 e 3.24 deixam claro que a distribuição do viés é assimétrica, tanto da estimativa do $\alpha$ quanto do $\lambda$. Todos os métodos de estimação se mostraram bons, pois não geram estimativas viesadas, e como é de se esperar, a medida que aumentamos o tamanho da série, mais concentrados são os valores do viés de $\hat{\alpha}$ e viés do $\hat{\lambda}$ em torno de zero. 


\begin{tabular}{ccccccccc}
\hline \hline & & \multicolumn{3}{c}{ Viés $(\hat{\alpha})$} & & \multicolumn{3}{c}{ Viés $(\hat{\lambda})$} \\
\cline { 9 - 10 } \cline { 7 - 9 } $\mathrm{N}$ & $\alpha$ & Y-W & MQC & MVC & & Y-W & MQC & MVC \\
\hline \multirow{2}{10}{100} & 0,3 & $-0,03038$ & $-0,02764$ & $-0,01593$ & & 0,03733 & 0,03016 & 0,01753 \\
& 0,5 & $-0,03128$ & $-0,02664$ & $-0,00929$ & & 0,05981 & 0,0402 & 0,01413 \\
& 0,7 & $-0,03912$ & $-0,03187$ & $-0,00759$ & & 0,12104 & 0,07558 & 0,01824 \\
200 & 0,3 & $-0,00376$ & $-0,0023$ & $-0,00171$ & & 0,00578 & 0,00184 & 0,00319 \\
& 0,5 & $-0,0158$ & $-0,0136$ & $-0,00118$ & & 0,02634 & 0,01763 & $-0,00221$ \\
& 0,7 & $-0,02126$ & $-0,01761$ & $-0,00125$ & & 0,07087 & 0,04748 & 0,00359 \\
500 & 0,3 & $-0,00426$ & $-0,00371$ & $-0,00268$ & & 0,0013 & $-0,00026$ & $-0,00089$ \\
& 0,5 & $-0,00593$ & $-0,00496$ & $-0,00222$ & & 0,01074 & 0,00704 & 0,00380 \\
& 0,7 & $-0,00787$ & $-0,00653$ & $-0,00116$ & & 0,02223 & 0,01319 & 0,00042 \\
\hline \hline
\end{tabular}

Tabela 3.3: Viés das estimações para o modelo $\operatorname{INAR(1)}(\lambda=1)$ baseado em 500 réplicas.

\begin{tabular}{ccccccccc}
\hline \hline & & \multicolumn{3}{c}{$\operatorname{EQM}(\hat{\alpha})$} & & \multicolumn{3}{c}{$\operatorname{EQM}(\hat{\lambda})$} \\
\cline { 3 - 4 } \cline { 8 - 9 } $\mathrm{N}$ & $\alpha$ & Y-W & MQC & MVC & & Y-W & MQC & MVC \\
\hline \multirow{2}{*}{100} & 0,3 & 0,01109 & 0,01117 & 0,00950 & & 0,03143 & 0,03179 & 0,02773 \\
& 0,5 & 0,00906 & 0,00885 & 0,00544 & & 0,04428 & 0,04359 & 0,02759 \\
& 0,7 & 0,00783 & 0,00737 & 0,00250 & & 0,08665 & 0,07928 & 0,03000 \\
200 & 0,3 & 0,00491 & 0,00497 & 0,00414 & & 0,01464 & 0,01480 & 0,01294 \\
& 0,5 & 0,00459 & 0,00459 & 0,00257 & & 0,02153 & 0,02133 & 0,01338 \\
& 0,7 & 0,00339 & 0,00323 & 0,00118 & & 0,03883 & 0,03626 & 0,01328 \\
500 & 0,3 & 0,00206 & 0,00206 & 0,00164 & & 0,00536 & 0,00540 & 0,00461 \\
& 0,5 & 0,00188 & 0,00187 & 0,00115 & & 0,00790 & 0,00787 & 0,00546 \\
& 0,7 & 0,00128 & 0,00127 & 0,00044 & & 0,01356 & 0,01333 & 0,00537 \\
\hline \hline
\end{tabular}

Tabela 3.4: Erro Quadrático Médio das estimações para o modelo INAR(1) $(\lambda=1)$ baseado em 500 réplicas.

A Tabela 3.3 é uma tabela resumo com o viés médio das estimativas. Como visto nas distribuições das estimativas e do viés, as estimativas obtidas por MVC apresentaram os menores valores médios de viés comparado às metodologias YW e MQC. As estimativas de MQC são mais assertivas que de YW para todos os valores tomados de $\alpha$, em todos tamanhos de série. Quanto maior o valor do $\alpha$ usado para gerar a série, maior é a evidência de que o estimador de MVC possui estimativas mais próximas do verdadeiro valor do parâmetro. Para $\alpha=0,7$ o víes do MVC chega a ser metade do víes médio dado pelos estimadores de YW e MQC. 


\begin{tabular}{|c|c|c|c|c|c|c|c|}
\hline \multirow[b]{2}{*}{$\mathrm{N}$} & \multirow[b]{2}{*}{$\alpha$} & \multicolumn{3}{|c|}{ Variância $(\hat{\alpha})$} & \multicolumn{3}{|c|}{$\operatorname{Variância}(\hat{\lambda})$} \\
\hline & & Y-W & MQC & MVC & Y-W & MQC & MVC \\
\hline \multirow[t]{3}{*}{100} & 0,3 & 0,01019 & 0,01042 & 0,00926 & 0,03010 & 0,03094 & 0,02748 \\
\hline & 0,5 & 0,00809 & 0,00816 & 0,00536 & 0,04078 & 0,04206 & 0,02745 \\
\hline & 0,7 & 0,00632 & 0,00637 & 0,00244 & 0,07214 & 0,07371 & 0,02972 \\
\hline \multirow[t]{3}{*}{200} & 0,3 & 0,00491 & 0,00498 & 0,00414 & 0,01463 & 0,01483 & 0,01295 \\
\hline & 0,5 & 0,00435 & 0,00442 & 0,00258 & 0,02088 & 0,02106 & 0,01341 \\
\hline & 0,7 & 0,00294 & 0,00292 & 0,00118 & 0,03388 & 0,03407 & 0,01329 \\
\hline \multirow[t]{3}{*}{500} & 0,3 & 0,00204 & 0,00205 & 0,00164 & 0,00537 & 0,00541 & 0,00461 \\
\hline & 0,5 & 0,00184 & 0,00185 & 0,00115 & 0,00780 & 0,00784 & 0,00545 \\
\hline & 0.7 & 0.00123 & 0,00123 & 0,00044 & 0.01309 & 0,01318 & 0.00539 \\
\hline
\end{tabular}

Tabela 3.5: Variância dos valores estimados para o modelo $\operatorname{INAR(1)}(\lambda=1)$ baseado em 500 réplicas.

Além de produzir estimativas melhores, o estimador de MVC apresenta estimativas mais concentradas, como se pode observar na Figura 3.21. A Tabela 3.5 mostra as variâncias obtidas em 500 réplicas de cada modelo gerado. Assim como o viés e o EQM, as variâncias obtidas por MVC são menores quando comparadas com as variâncias calculadas das estimativas de YW e MQC.

Dando sequência à análise de simulações dos modelos INAR, apresentados na Tabela 3.2, são mostrados em seguida os modelos INAR de ordem igual a dois. A escolha dos parâmetros para simulação foi feita de maneira que a soma dos parâmetros não superasse 0,8 . Seguiu-se com a mesma estrutura de apresentação, primeiro é apresentado uma trajetória simulada, em seguida os resultados de estimações em cada método. O comparativo do viés e EQM em cada metodologia de estimação também é mostrado. 
Processo INAR(2): $X_{t}=0,3 \circ X_{t-1}+0,3 \circ X_{t-2}$

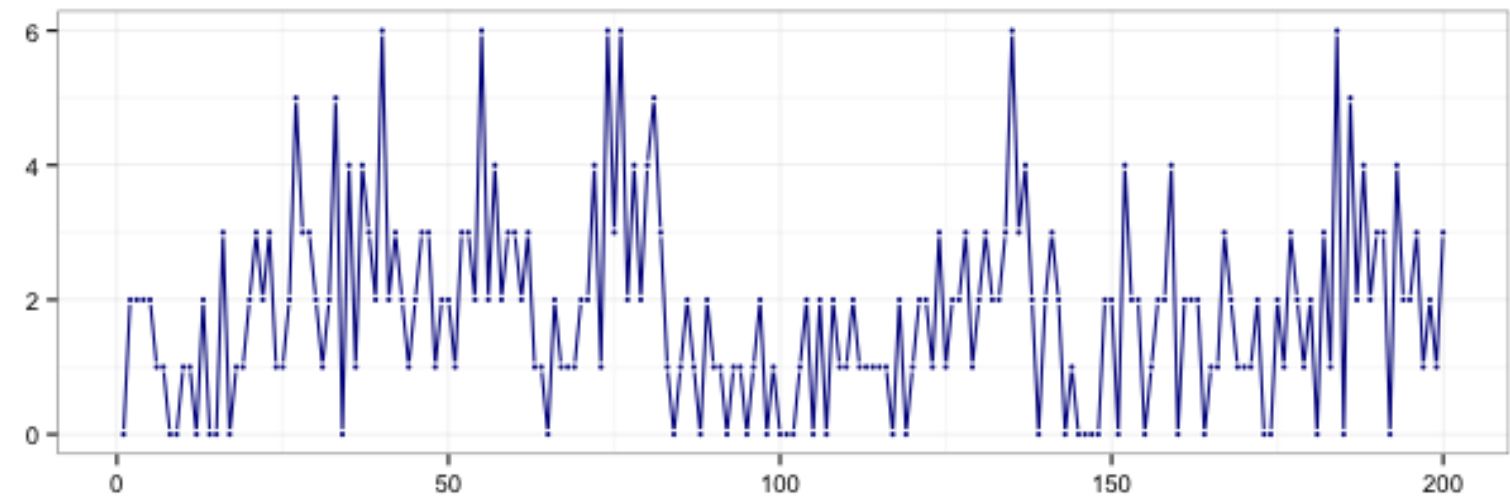

Figura 3.25: Gráfico de uma série simulada, $\operatorname{INAR(2):~} \alpha_{1}=0,3, \alpha_{2}=0,3$ e $\lambda=1$.

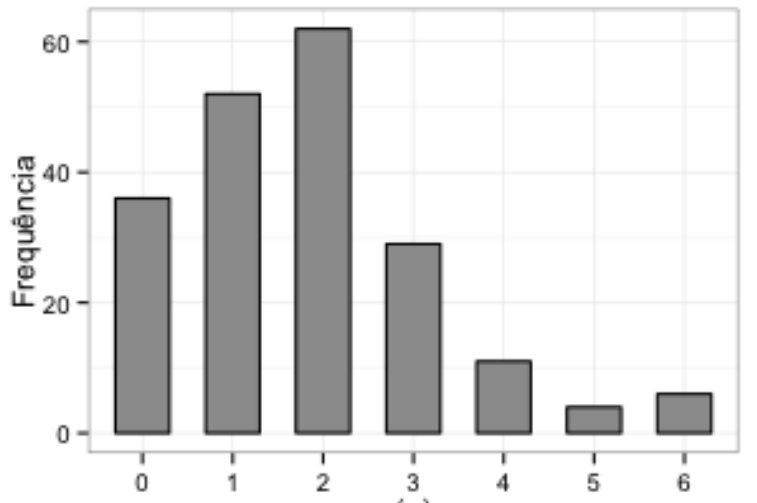

(a)

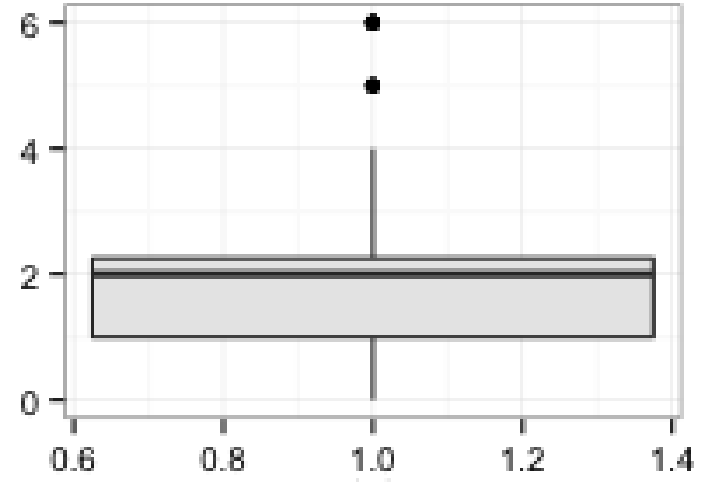

(b)

Figura 3.26: Histograma e boxplot da série simulada, INAR(2): $\alpha_{1}=0,3, \alpha_{2}=0,3$ e $\lambda=1$.
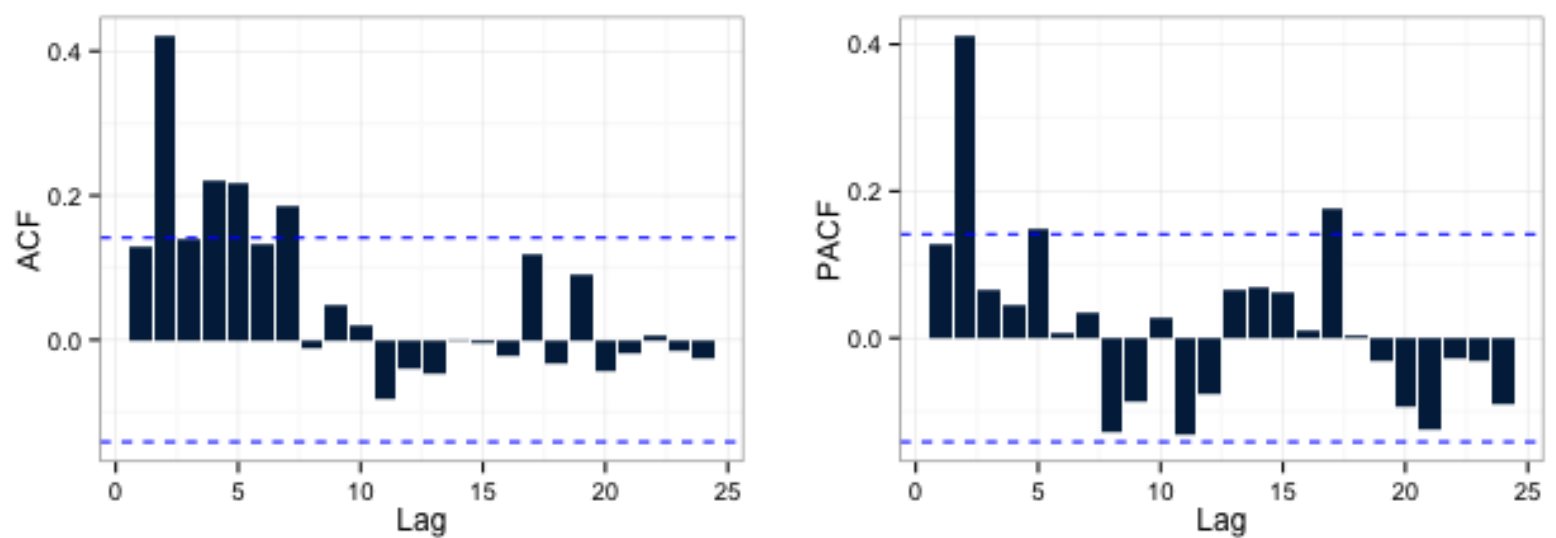

Figura 3.27: Autocorrelação e Autocorrelação parcial amostrais $\operatorname{INAR(2):~} \alpha_{1}=0,3, \alpha_{2}=0,3$ e $\lambda=1$.

A Figura 3.25 apresenta um exemplo da trajetória gerada pelo processo $\operatorname{INAR}(2) X_{t}=0,3 \circ$ $X_{t-1}+0,3 \circ X_{t-2}+I_{t}$ com a inovação $I_{t}$ dada pela Poisson(1). Temos o histograma na Figura 3.26, assim como o boxplot; ambos acentuam a não simetria dos dados, característica de uma Poisson com parâmetro $\lambda$ pequeno. 
A Figura 3.27 mostra os gráficos de autocorrelação e autocorrelação parcial; estes gráficos sugerem que a trajetória gerada possui autocorrelação parcial de ordem dois diferente de zero, o que é esperado, já que as trajetórias foram obtidas através de um processo INAR(2).

A análise de simulações no caso de um modelo $\operatorname{INAR(2)~é~uma~extensão~do~} \operatorname{INAR}(1)$ porque temos um parâmetro a mais com que se preocupar. A análise desse primeiro parâmetro é mostrada na Figura 3.28, que apresenta o boxplot das estimativas do $\alpha_{1}$ para cada uma das metodologias de estimação sugeridas, seguindo com o segundo parâmetro $\alpha_{2}$ na Figura 3.29 e o parâmetro da distribuição de inovação $\lambda$ na Figura 3.30 .

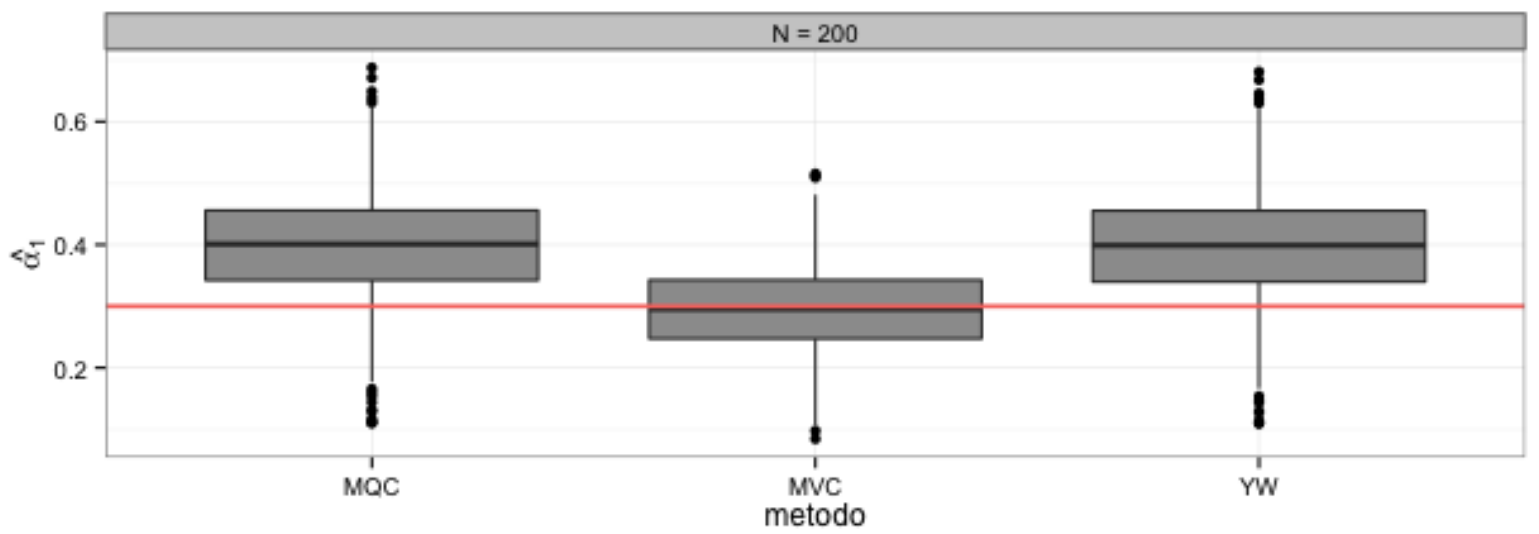

Figura 3.28: Boxplot das estimativas de $\alpha_{1}$ para o modelo gerado por $X_{t}=0,3 \circ X_{t-1}+0,3 \circ X_{t-2}+I_{t}$.

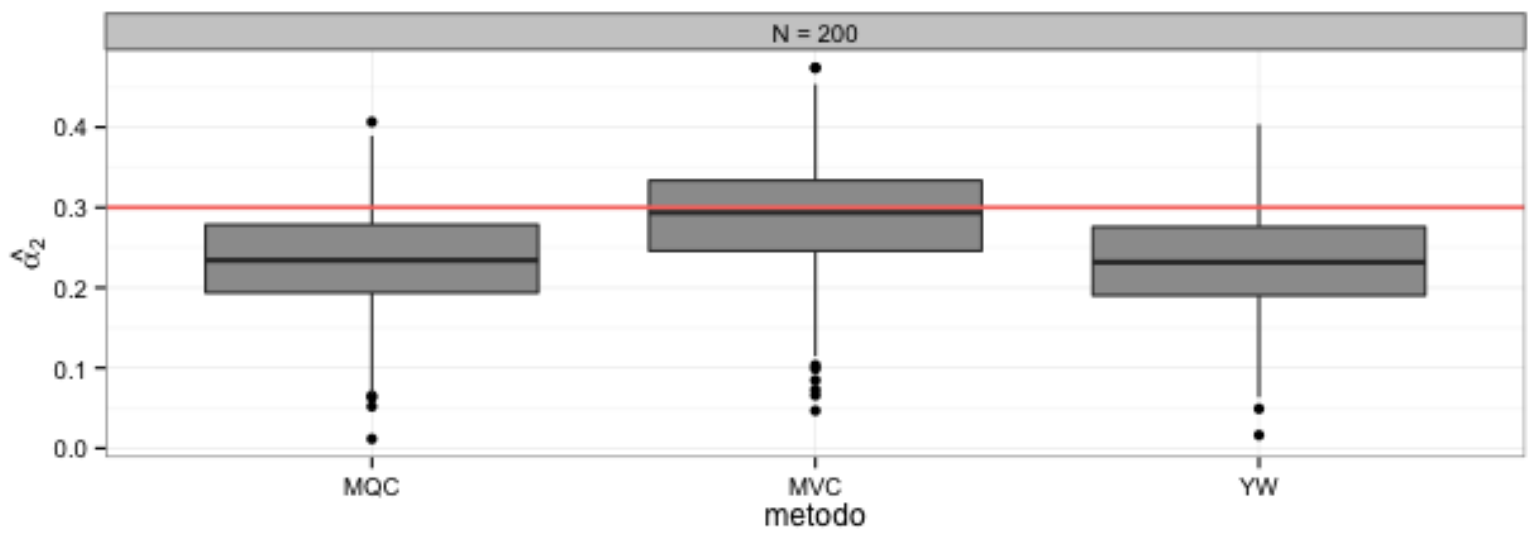

Figura 3.29: Boxplot das estimativas de $\alpha_{2}$ para o modelo gerado por $X_{t}=0,3 \circ X_{t-1}+0,3 \circ X_{t-2}+I_{t}$.

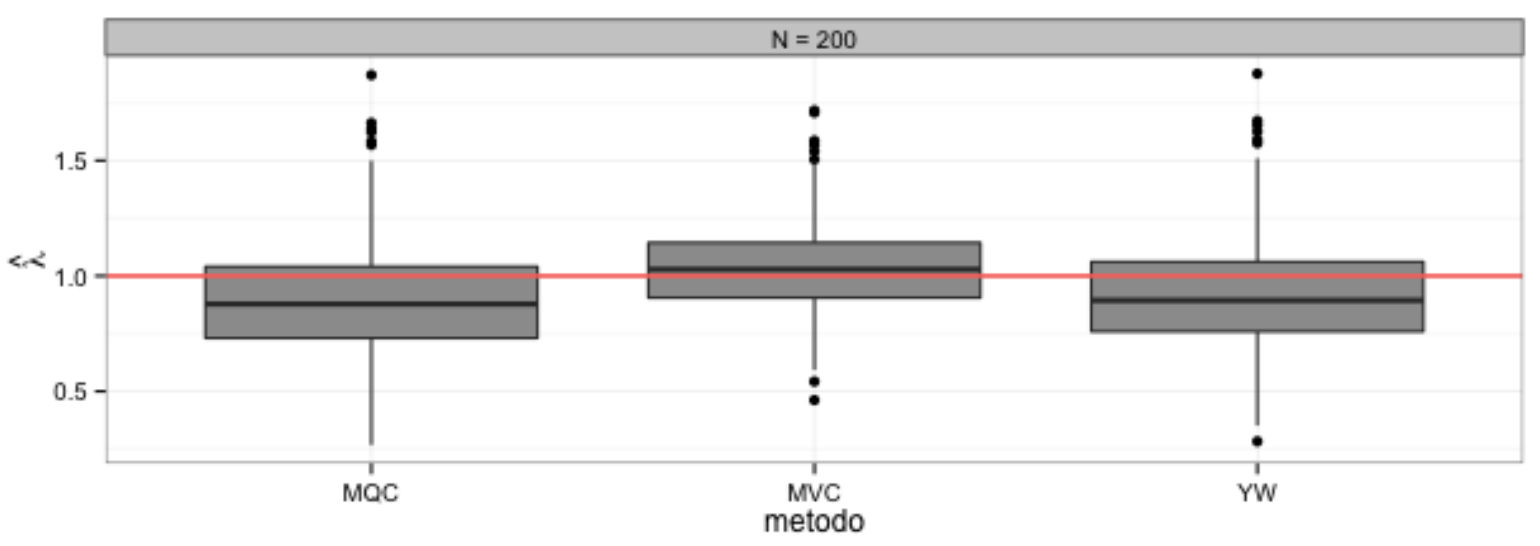

Figura 3.30: Boxplot das estimativas de $\lambda$ para o modelo gerado por $X_{t}=0,3 \circ X_{t-1}+0,3 \circ X_{t-2}+I_{t}$. 
Os vieses de cada parâmetro estimado são apresentados nas Figuras 3.31. É claro, tanto através dos gráficos boxplot quanto pelo histograma alisado do viés, que o método de máxima verossimilhança condicional apresenta melhor desempenho comparado com os demais, para os três parâmetros estimados. Isso se deve principalmente porque a informação sobre a distribuição que assumimos é contemplada no método de máxima verossimilhança, diferentemente dos demais. A consideração da distribuição na hora de estimar tem seu peso aumentado, dado que o parâmetro usado como a média da Poisson $(\lambda)$ é relativamente baixo, caso em que a distribuição normal não possui boa aproximação.

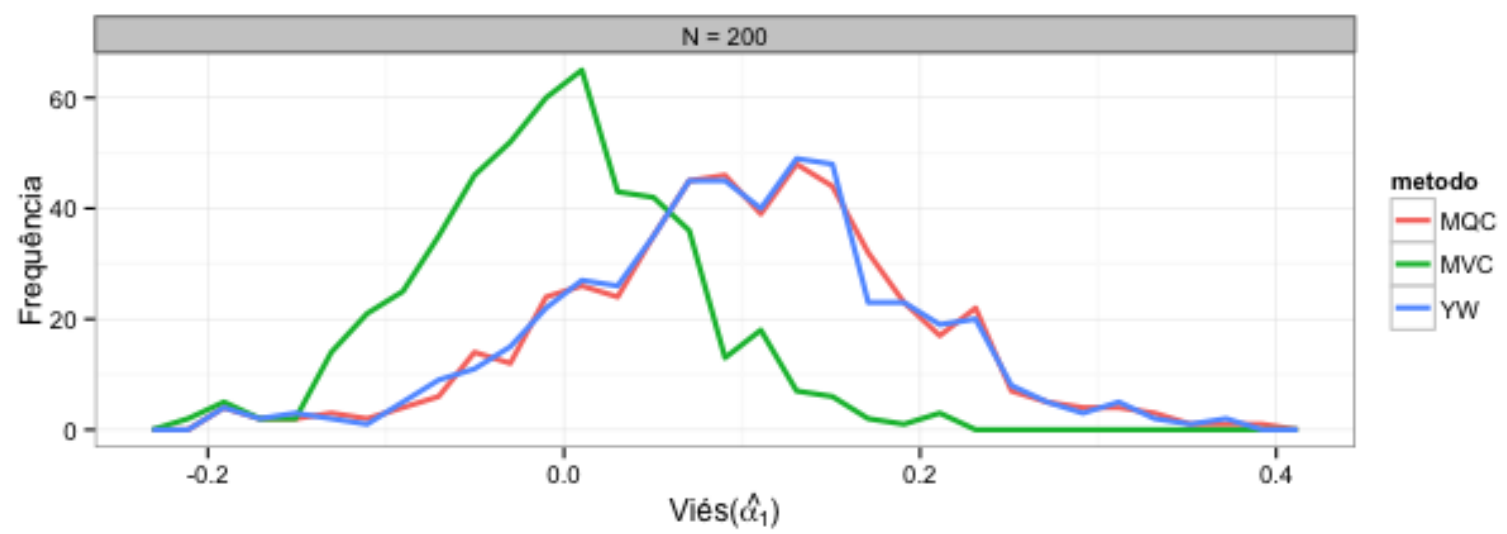

Figura 3.31: Histograma alisado do viés de $\hat{\alpha_{1}}$ para o modelo gerado por $X_{t}=0,3 \circ X_{t-1}+0,3 \circ X_{t-2}+I_{t}$.

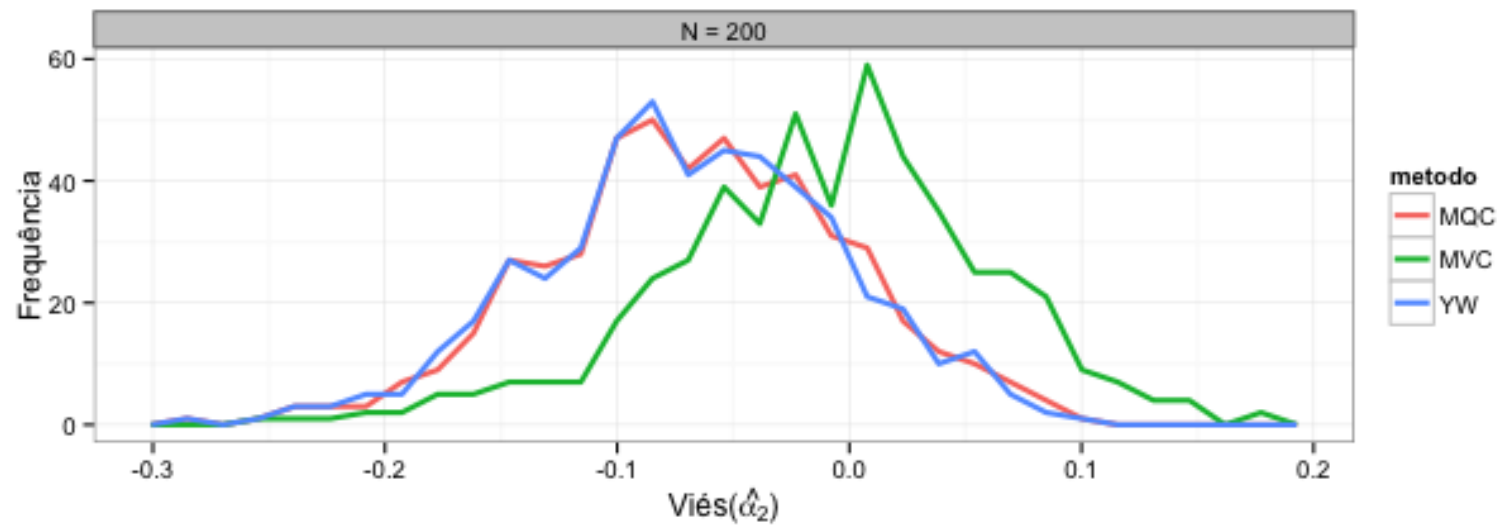

Figura 3.32: Histograma alisado do viés de $\hat{\alpha_{2}}$ para o modelo gerado por $X_{t}=0,3 \circ X_{t-1}+0,3 \circ X_{t-2}+I_{t}$.

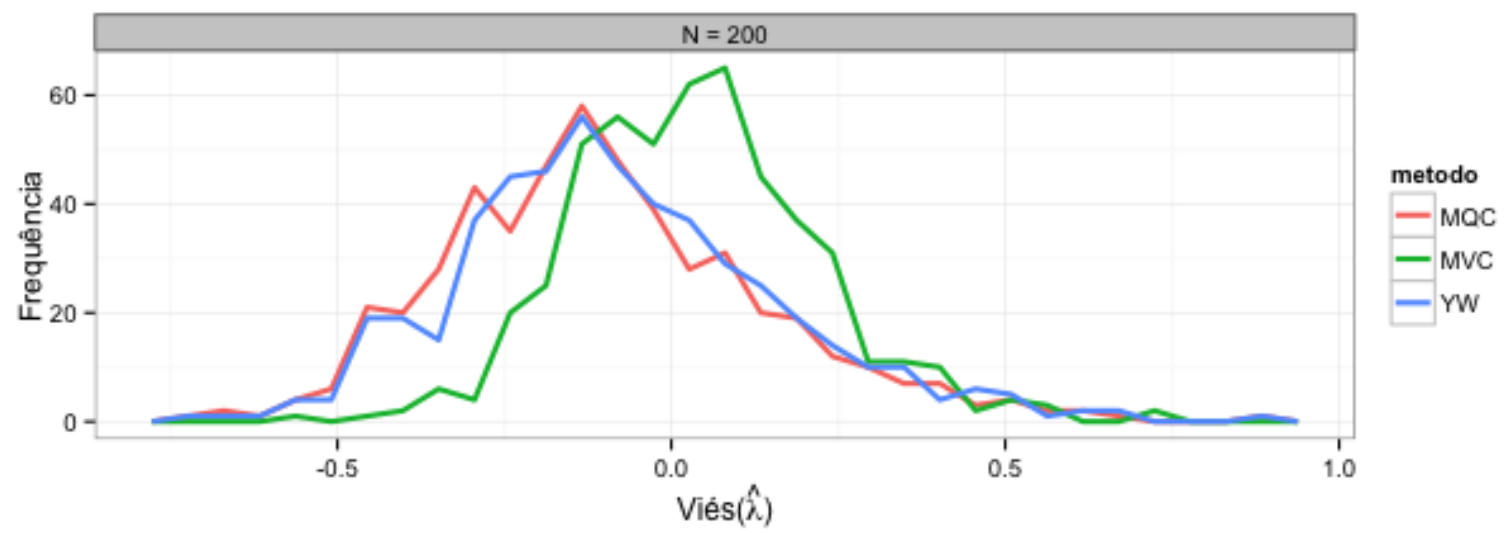

Figura 3.33: Histograma alisado do viés de $\hat{\lambda}$ para o modelo gerado por $X_{t}=0,3 \circ X_{t-1}+0,3 \circ X_{t-2}+I_{t}$. 
Processo INAR(2): $X_{t}=0,3 \circ X_{t-1}+0,5 \circ X_{t-2}$

Assim como no processo simulado anteriormente, tomando $\alpha_{2}=0,5$ temos um exemplo de um processo INAR(2) mostrado na Figura 3.34. Em seguida, o histograma dos valores simulados e boxplot na Figura 3.35. O comportamento da autocorrelação e autocorrelação parcial dada na Figura 3.36, apresenta as características do processo INAR de ordem dois, com autocorrelações parciais significantes nas ordens 1 e 2 e decaimento exponencial no gráfico da autocorrelação.

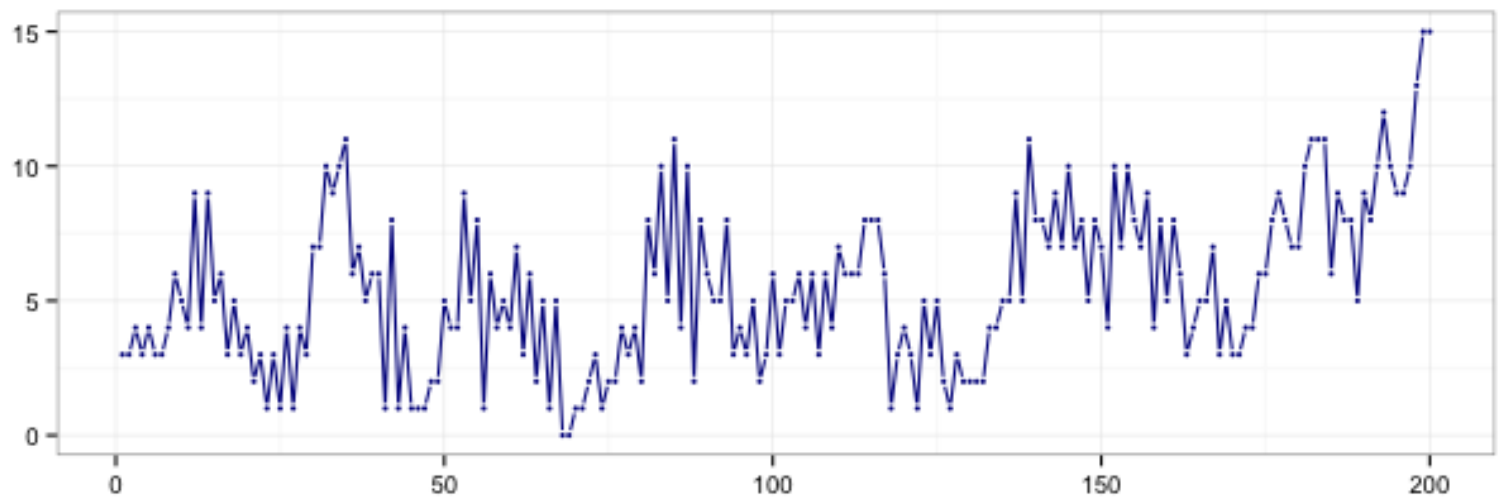

Figura 3.34: Gráfico de uma série simulada, $\operatorname{INAR(2):~} \alpha_{1}=0,3, \alpha_{2}=0,5$ e $\lambda=1$.

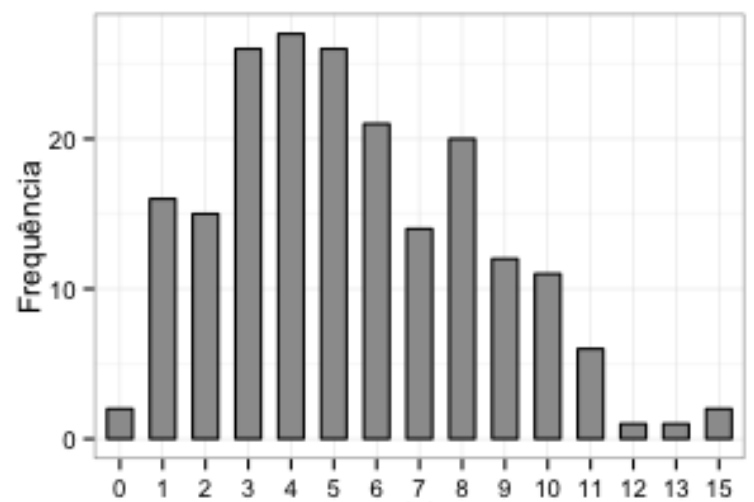

(a)

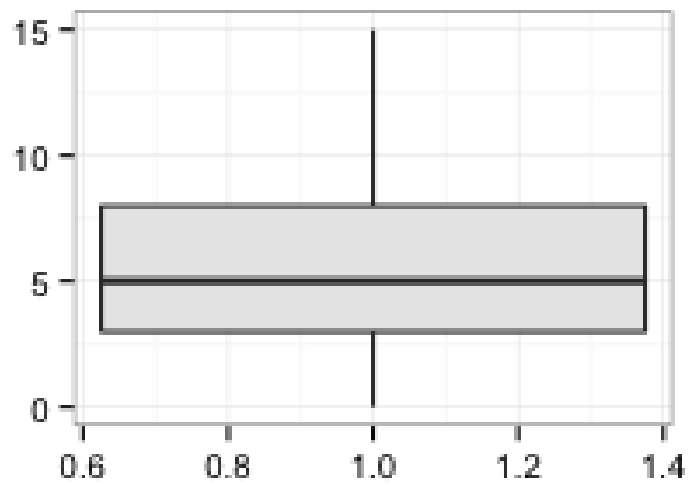

(b)

Figura 3.35: Histograma e boxplot da série simulada, INAR(2): $\alpha_{1}=0,3, \alpha_{2}=0,5$ e $\lambda=1$.
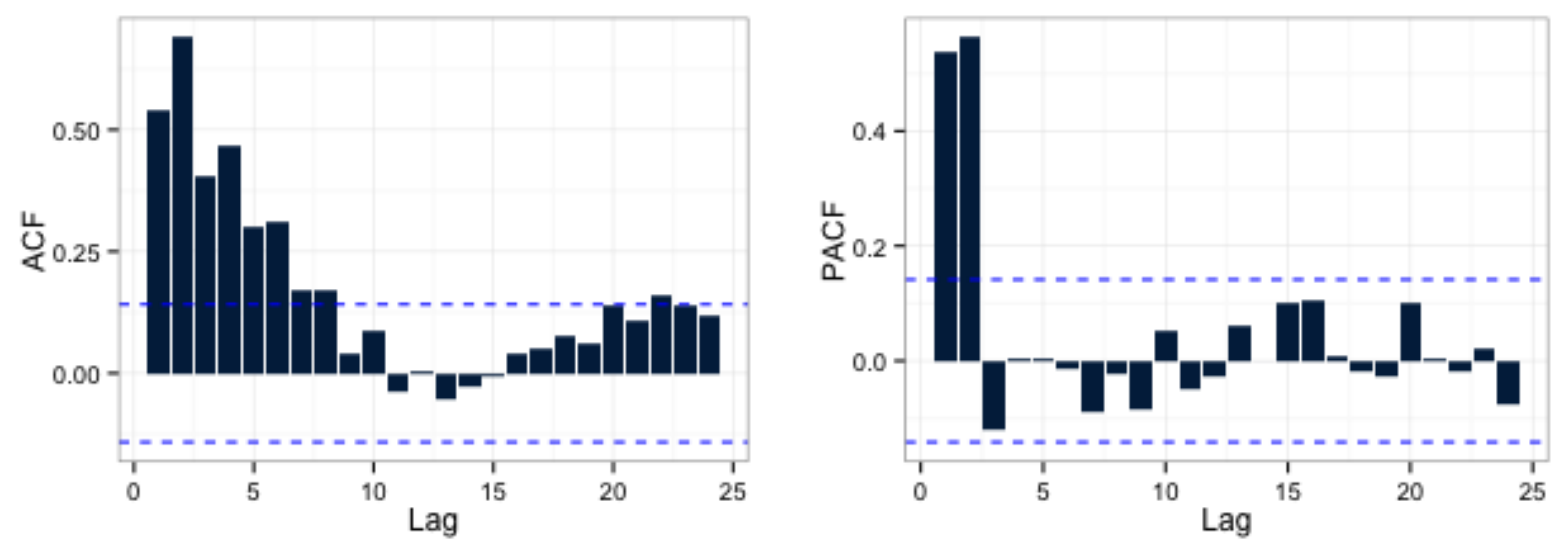

Figura 3.36: Autocorrelação e Autocorrelação parcial amostrais INAR(2): $\alpha_{1}=0,3, \alpha_{2}=0,5$ e $\lambda=1$. 


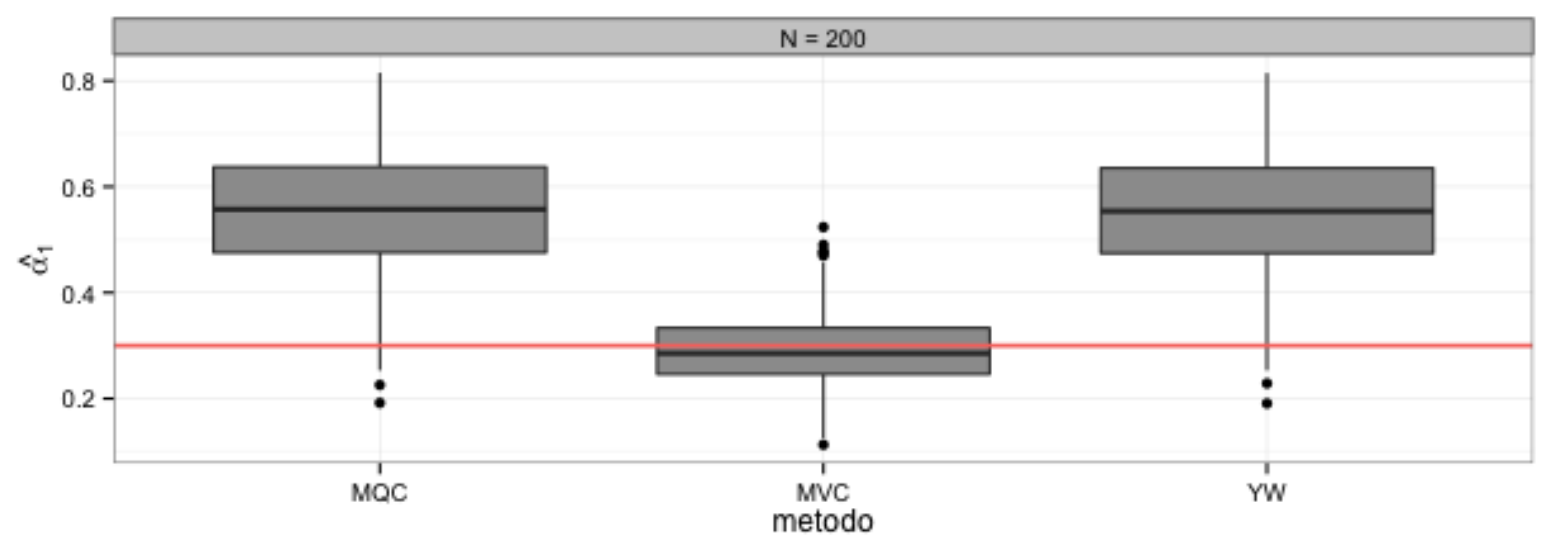

Figura 3.37: Boxplot das estimativas de $\alpha_{1}$ para o modelo gerado por $X_{t}=0,3 \circ X_{t-1}+0,5 \circ X_{t-2}+I_{t}$.

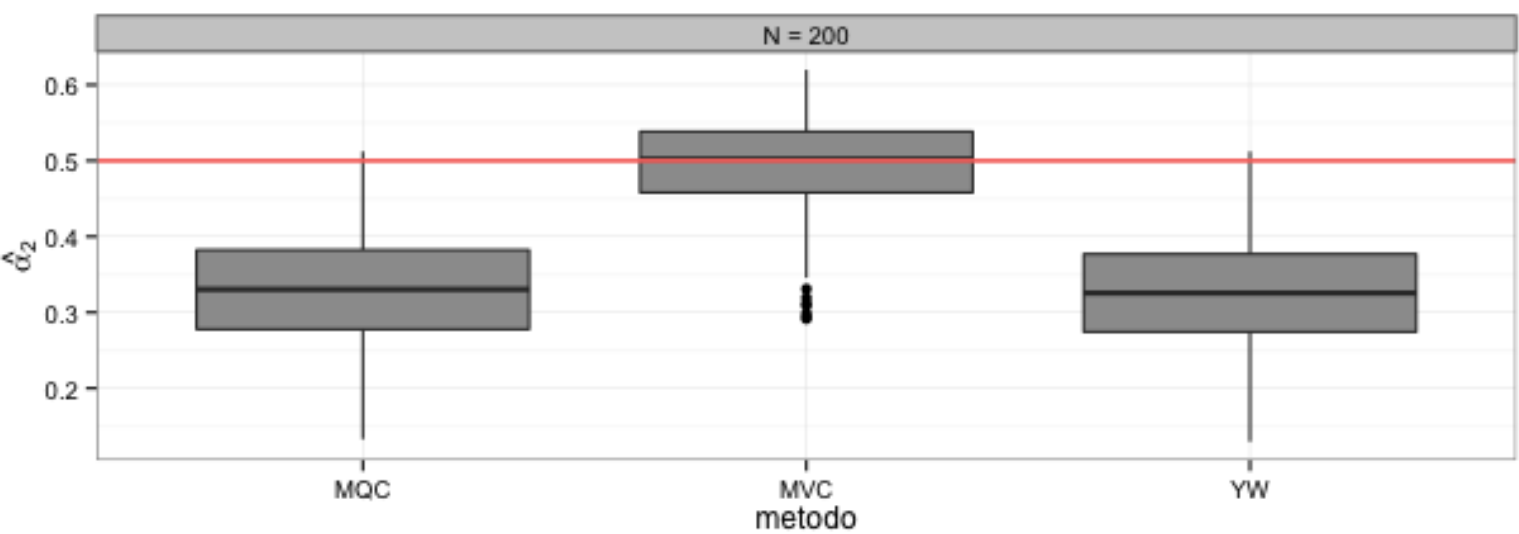

Figura 3.38: Boxplot das estimativas de $\alpha_{2}$ para o modelo gerado por $X_{t}=0,3 \circ X_{t-1}+0,5 \circ X_{t-2}+I_{t}$.

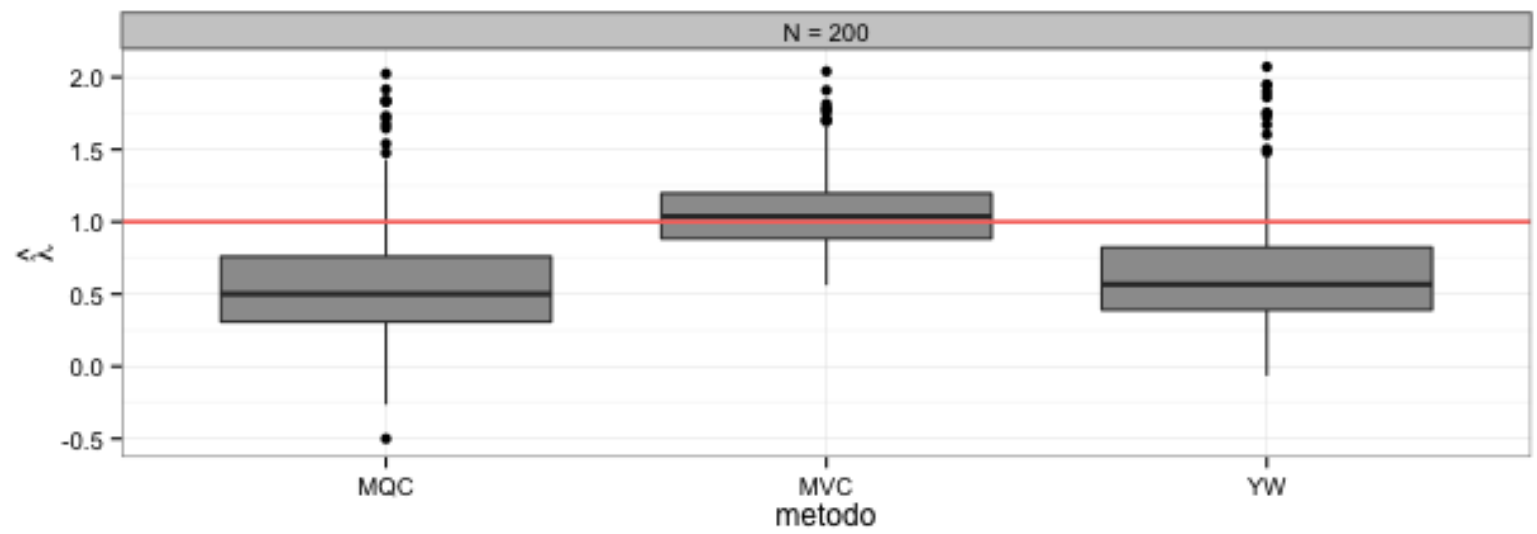

Figura 3.39: Boxplot das estimativas de $\lambda$ para o modelo gerado por $X_{t}=0,3 \circ X_{t-1}+0,5 \circ X_{t-2}+I_{t}$.

A análise segue com o destaque para a metodologia de máxima verossimilhança. Novamente é a metodologia com melhor resultado de estimação, isso é claro nas Figuras 3.37, 3.38 e 3.39 que mostram que o valor mediano da estimativa por máxima verossimilhança está mais próximo do verdadeiro valor do parâmetro, comparado à estimação de mínimos quadrados condicionais ou pelas equações de Yule-Walker. 


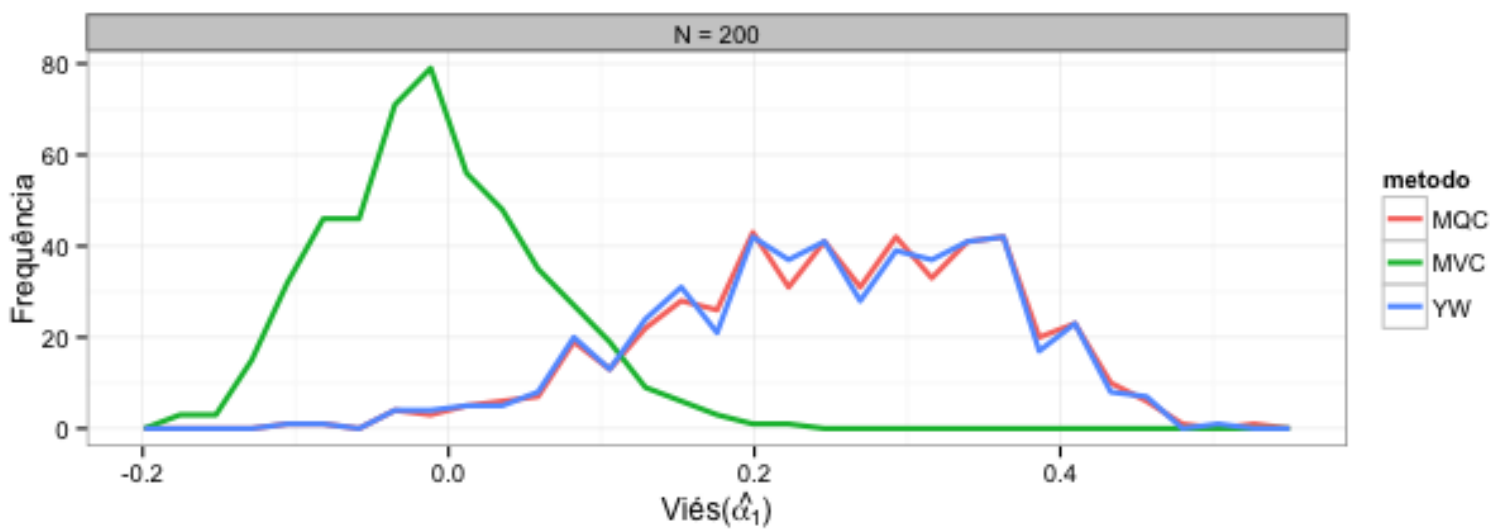

Figura 3.40: Histograma alisado do viés de $\hat{\alpha_{1}}$ para o modelo gerado por $X_{t}=0,3 \circ X_{t-1}+0,5 \circ X_{t-2}+I_{t}$.

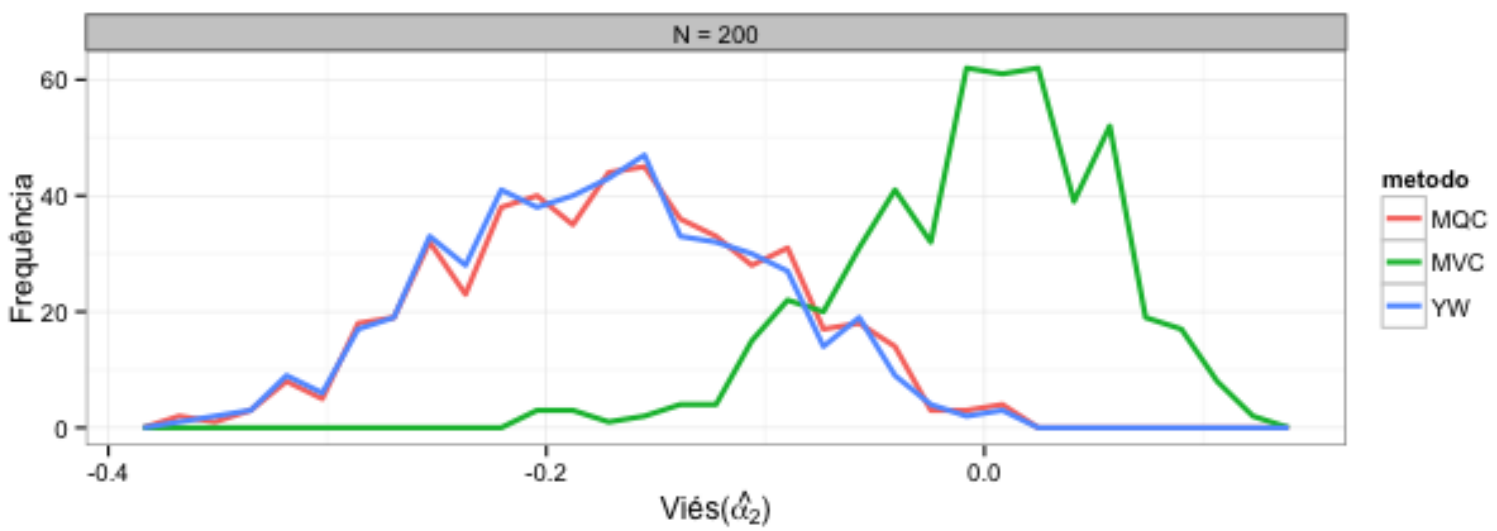

Figura 3.41: Histograma alisado do viés de $\hat{\alpha_{2}}$ para o modelo gerado por $X_{t}=0,3 \circ X_{t-1}+0,5 \circ X_{t-2}+I_{t}$.

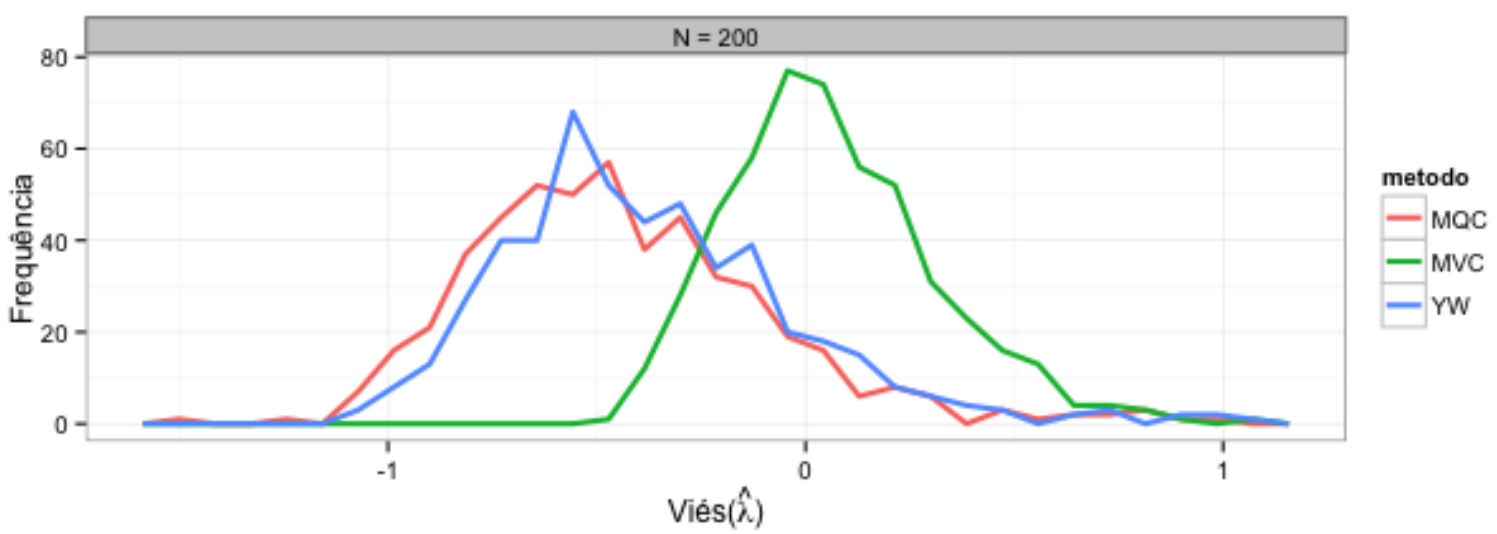

Figura 3.42: Histograma alisado do viés de $\hat{\lambda}$ para o modelo gerado por $X_{t}=0,3 \circ X_{t-1}+0,5 \circ X_{t-2}+I_{t}$.

A diferença entre os métodos de estimação é mais uma vez destacada nos histogramas alisados dos vieses exibidos nas Figuras 3.40, 3.41 e 3.42. A proximidade das estimativas dadas por YW e MQC são devido à propriedade do estimador de MQC, que à medida que o tamanho da série aumenta, o estimador MQC torna-se equivalente ao estimador YW. 
Processo INAR(2): $X_{t}=0,5 \circ X_{t-1}+0,3 \circ X_{t-2}$

Por fim o último processo INAR de ordem igual a dois simulado com $\alpha_{1}=0,5$ e $\alpha_{2}=0,3$ e $\lambda=1$, tem uma trajetória apresentada na Figura 3.43.

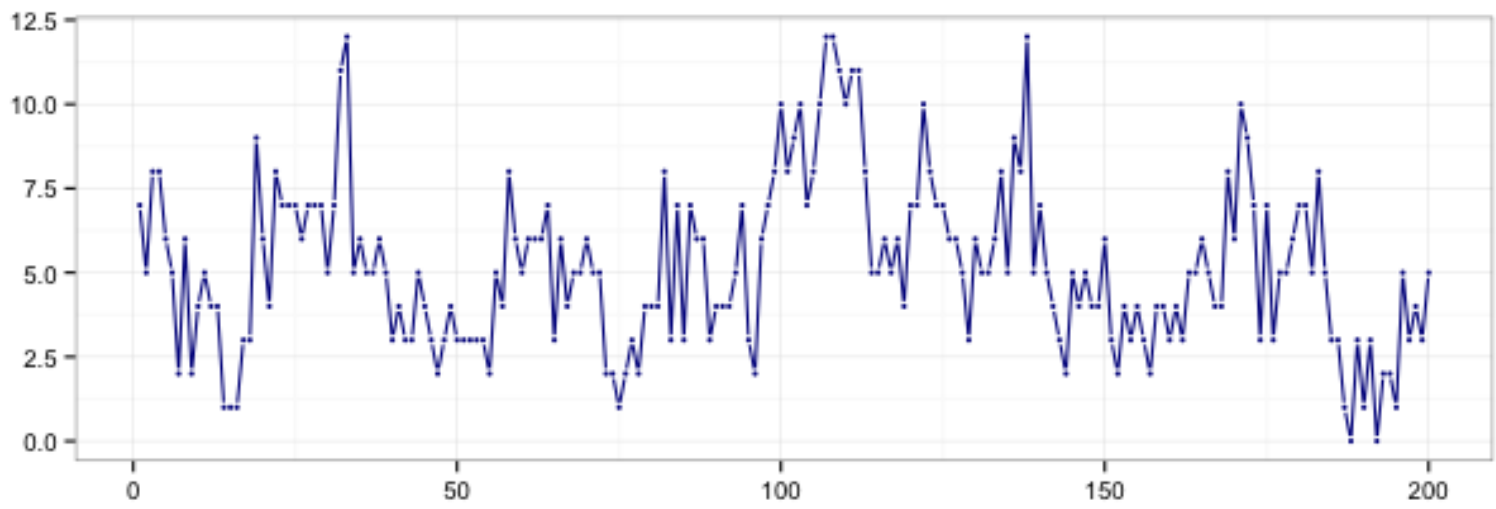

Figura 3.43: Gráfico de uma série simulada, $\operatorname{INAR(2):~} \alpha_{1}=0,5, \alpha_{2}=0,3$ e $\lambda=1$.

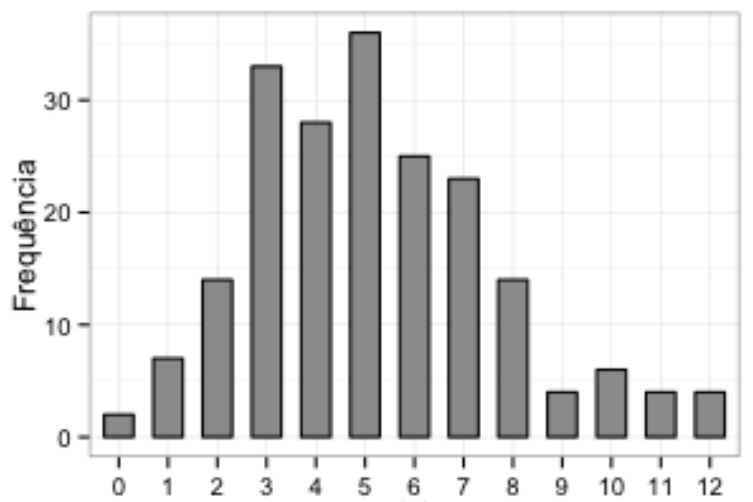

(a)

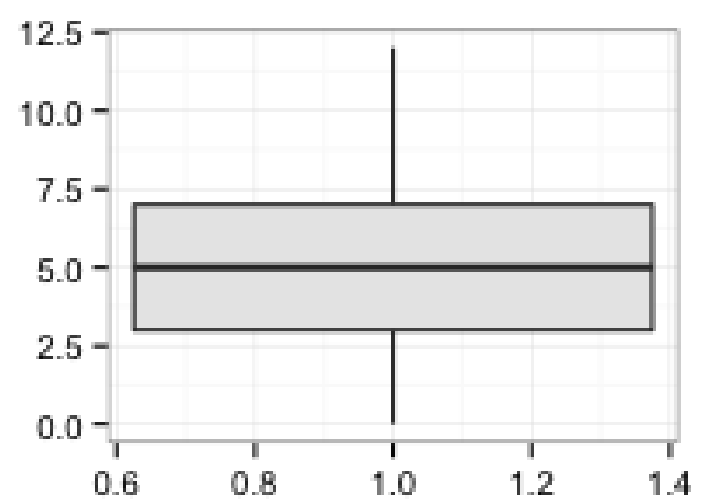

(b)

Figura 3.44: Histograma e boxplot da série simulada, INAR(2): $\alpha_{1}=0,5, \alpha_{2}=0,3$ e $\lambda=1$.
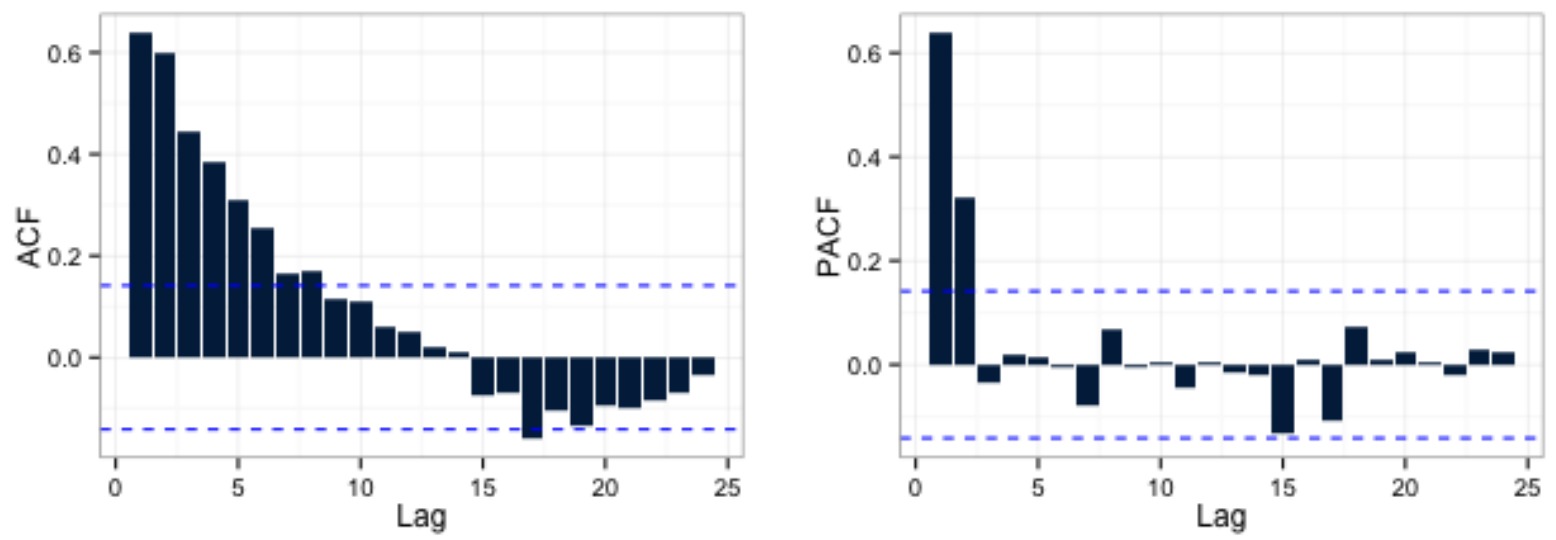

Figura 3.45: Autocorrelação e Autocorrelação parcial amostrais INAR(2): $\alpha_{1}=0,5, \alpha_{2}=0,3$ e $\lambda=1$.

A Figura 3.44 apresenta o histograma (a) e o boxplot (b) da série gerada. Os comportamentos da autocorrelação e autocorrelação parcial dados na Figura 3.45 correspondem com o esperado para este modelo: decaimento exponencial na $\mathrm{ACF}$ e as duas primeiras ordens na PACF significantes. 


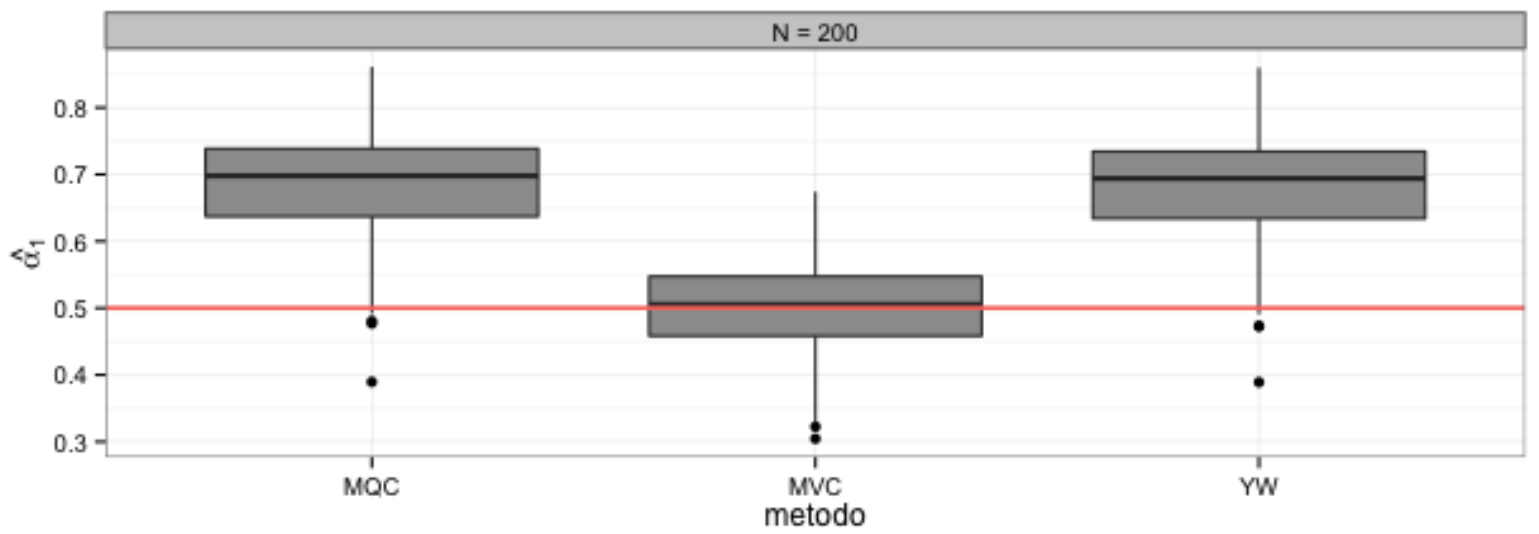

Figura 3.46: Boxplot das estimativas de $\alpha_{1}$ para o modelo gerado por $X_{t}=0,5 \circ X_{t-1}+0,3 \circ X_{t-2}+I_{t}$.

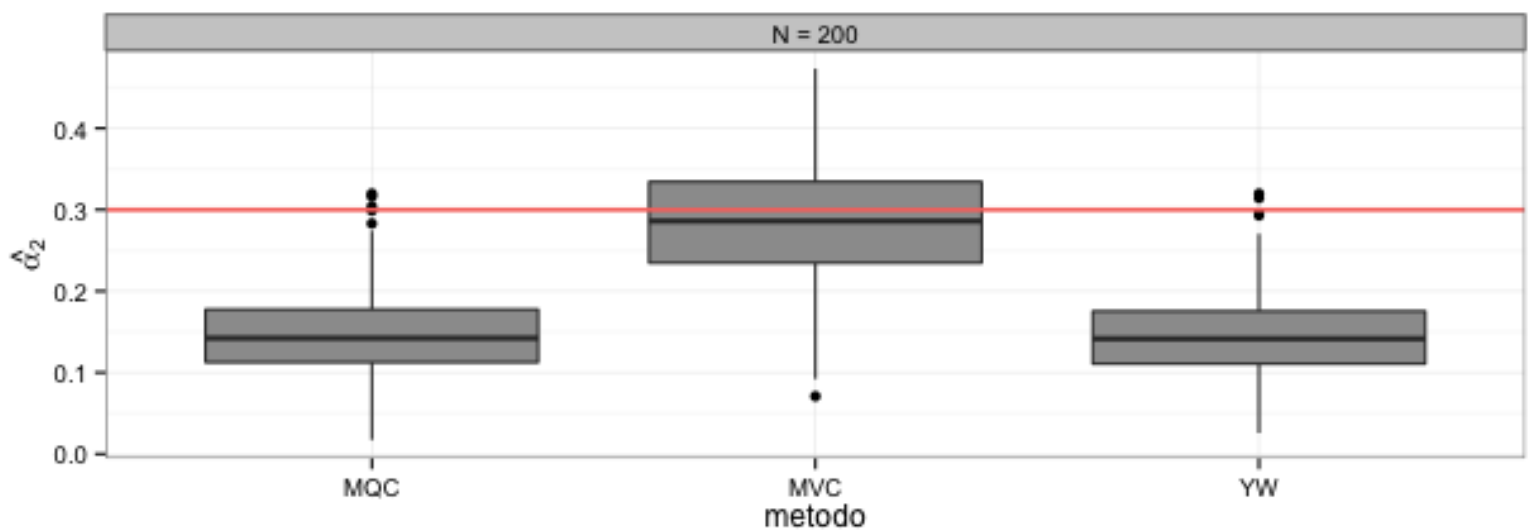

Figura 3.47: Boxplot das estimativas de $\alpha_{2}$ para o modelo gerado por $X_{t}=0,5 \circ X_{t-1}+0,3 \circ X_{t-2}+I_{t}$.

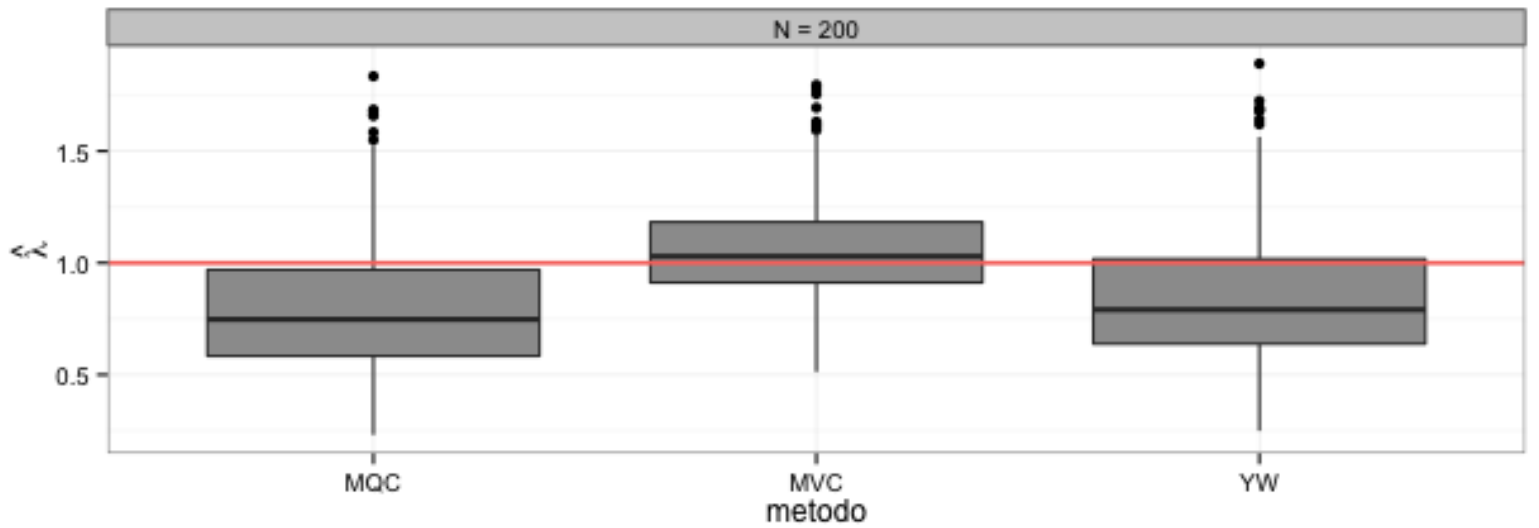

Figura 3.48: Boxplot das estimativas de $\lambda$ para o modelo gerado por $X_{t}=0,5 \circ X_{t-1}+0,3 \circ X_{t-2}+I_{t}$. 


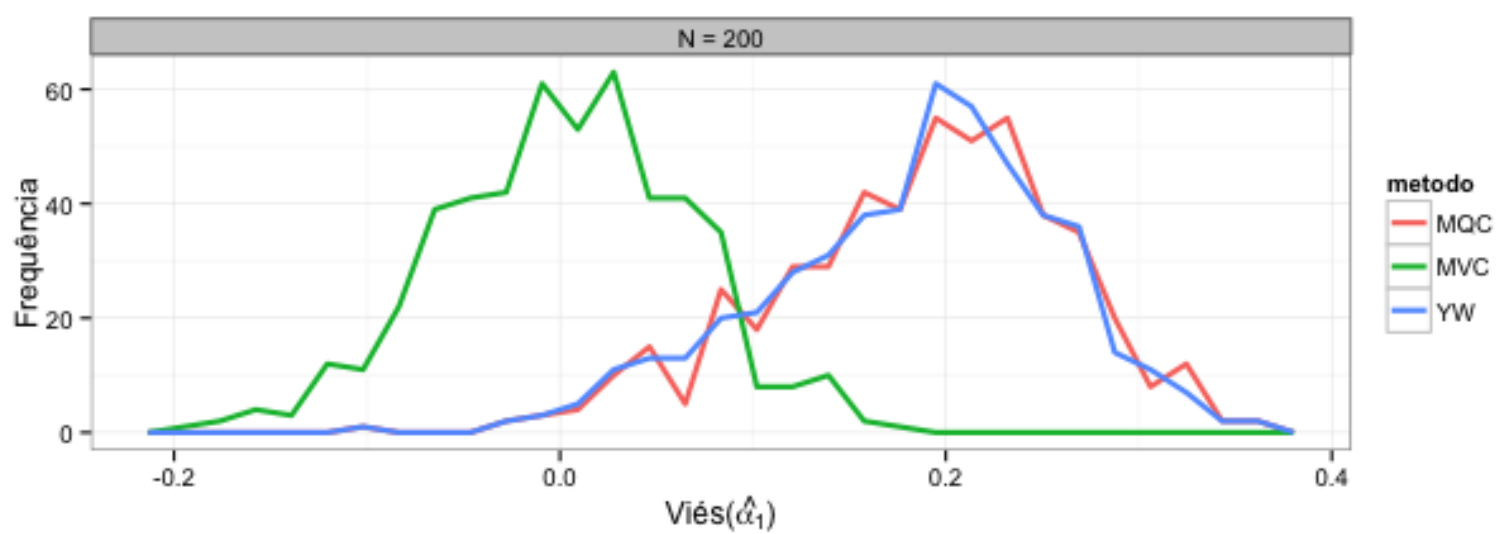

Figura 3.49: Histograma alisado do viés de $\hat{\alpha_{1}}$ para o modelo gerado por $X_{t}=0,5 \circ X_{t-1}+0,3 \circ X_{t-2}+I_{t}$.

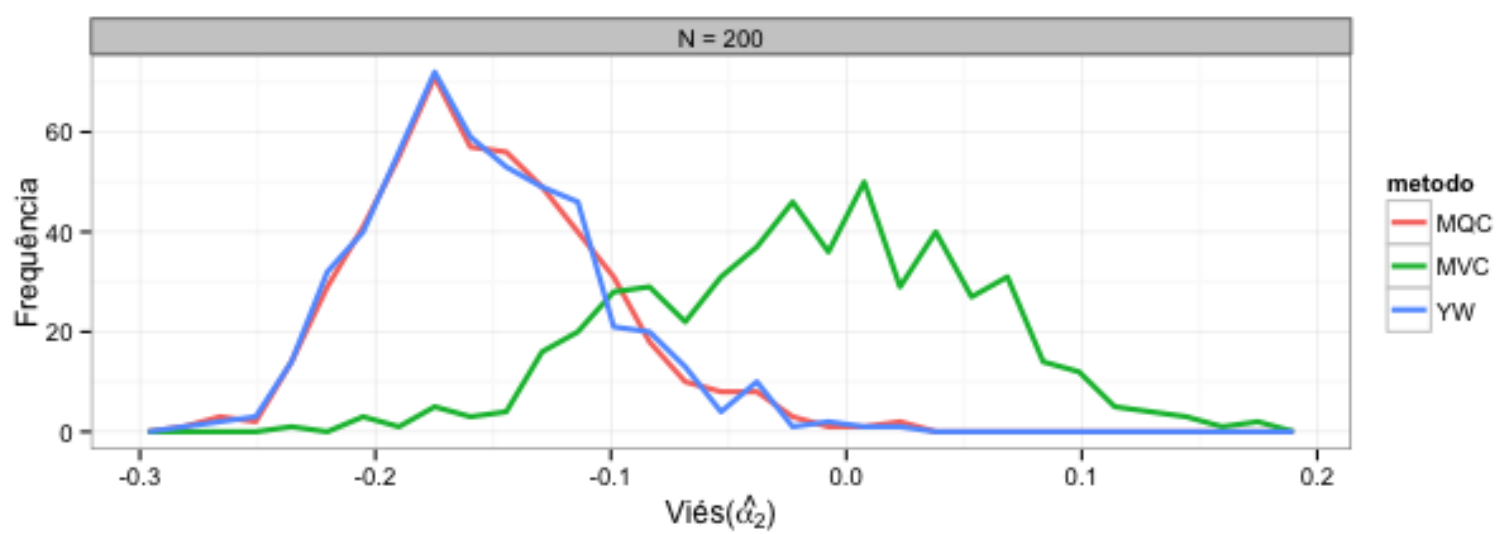

Figura 3.50: Histograma alisado do viés de $\hat{\alpha_{2}}$ para o modelo gerado por $X_{t}=0,5 \circ X_{t-1}+0,3 \circ X_{t-2}+I_{t}$.

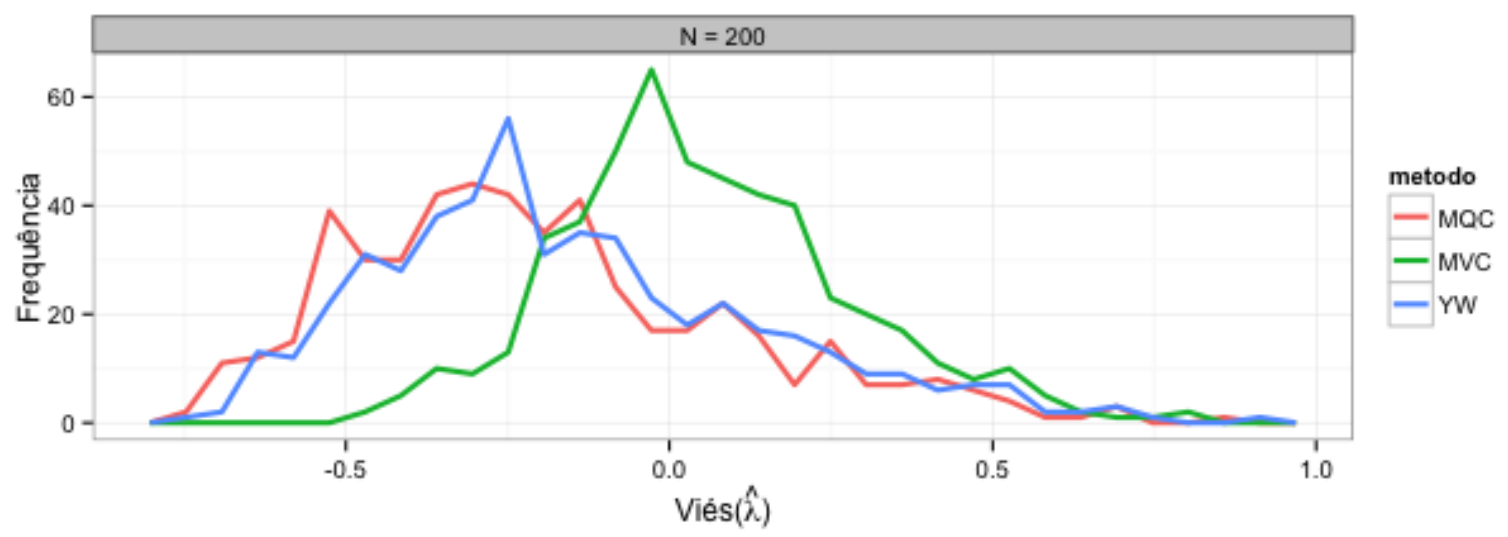

Figura 3.51: Histograma alisado do viés de $\hat{\lambda}$ para o modelo gerado por $X_{t}=0,5 \circ X_{t-1}+0,3 \circ X_{t-2}+I_{t}$.

Novamente a metodologia de máxima verossimilhança apresenta ganho sobre as demais, mostrando que tem estimativas mais próximas do parâmetro usado como referência. Tanto o boxplot das estimativas de cada metodologia, mostrados nas Figuras 3.46 a 3.48, quanto os vieses das estimativas mostrados nas Figuras 3.49 a 3.51, exibem o quanto a estimativa de MVC é mais próxima do verdadeiro valor do parâmetro. 


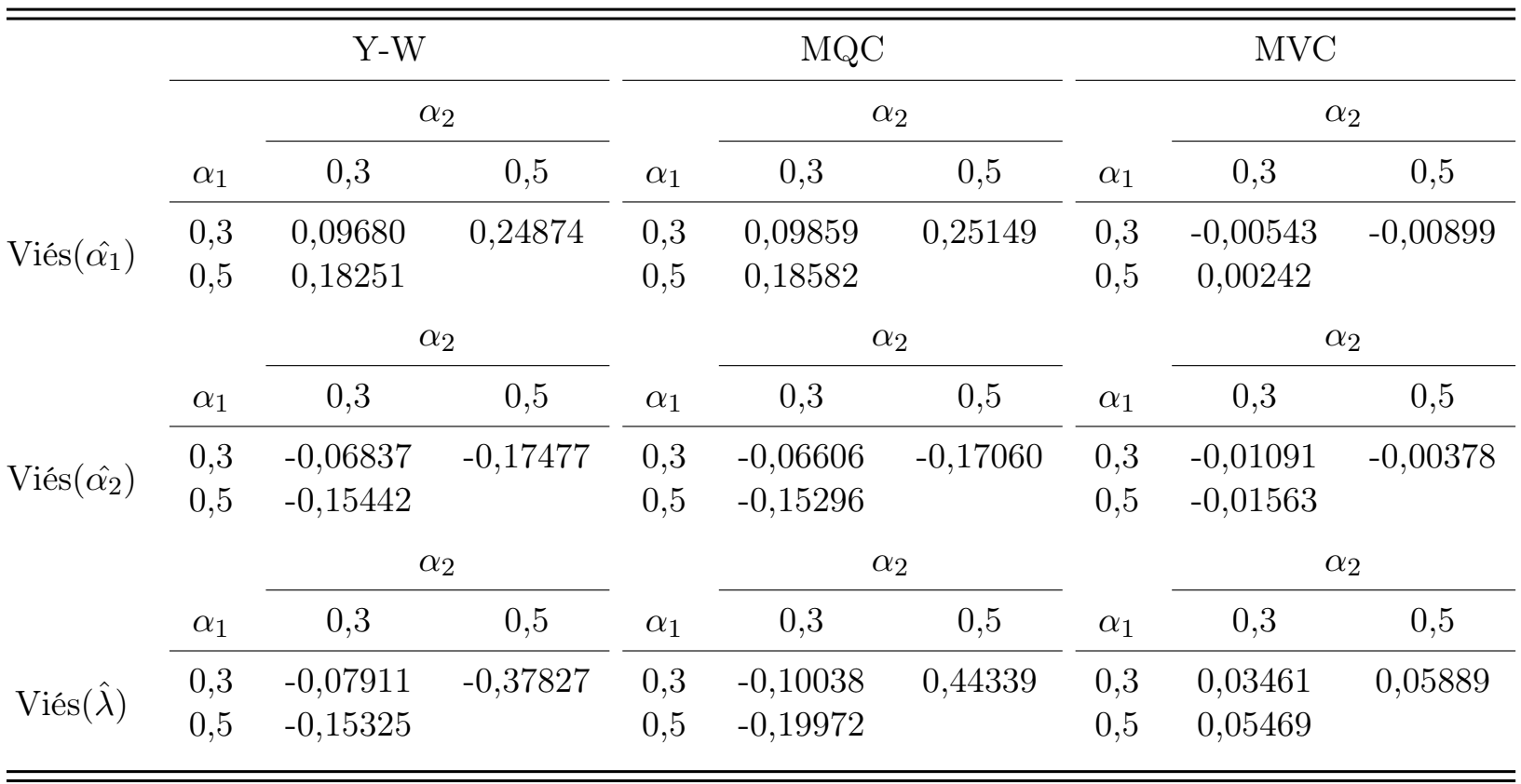

Tabela 3.6: Viés das estimativas dos parâmetros do modelo $\operatorname{INAR(2)~}(\lambda=1)$ baseado em 500 réplicas e $N=200$.

\begin{tabular}{|c|c|c|c|c|c|c|c|c|c|}
\hline & \multicolumn{3}{|c|}{$\mathrm{Y}-\mathrm{W}$} & \multicolumn{3}{|c|}{ MQC } & \multicolumn{3}{|c|}{ MVC } \\
\hline & \multirow[b]{2}{*}{$\alpha_{1}$} & \multicolumn{2}{|c|}{$\alpha_{2}$} & & \multicolumn{2}{|c|}{$\alpha_{2}$} & & \multicolumn{2}{|c|}{$\alpha_{2}$} \\
\hline & & 0,3 & 0,5 & $\alpha_{1}$ & 0,3 & 0,5 & $\alpha_{1}$ & 0,3 & 0,5 \\
\hline \multirow{2}{*}{$\operatorname{EQM}\left(\hat{\alpha_{1}}\right)$} & 0,3 & 0,01840 & 0,07381 & 0,3 & 0,01882 & 0,07533 & 0,3 & 0,00510 & 0,00465 \\
\hline & 0,5 & 0,03898 & & 0,5 & 0,04022 & & 0,5 & 0,00399 & \\
\hline \multirow{6}{*}{$\operatorname{EQM}\left(\hat{\alpha_{2}}\right)$} & \multirow[b]{2}{*}{$\alpha_{1}$} & \multicolumn{2}{|c|}{$\alpha_{2}$} & \multirow[b]{2}{*}{$\alpha_{1}$} & \multicolumn{2}{|c|}{$\alpha_{2}$} & \multirow[b]{2}{*}{$\alpha_{1}$} & \multicolumn{2}{|c|}{$\alpha_{2}$} \\
\hline & & 0,3 & 0,5 & & 0,3 & 0,5 & & 0,3 & 0,5 \\
\hline & 0,3 & 0,00885 & 0,03571 & 0,3 & 0,00861 & 0,03445 & 0,3 & 0,00497 & 0,00344 \\
\hline & 0,5 & 0,02615 & & 0,5 & 0,02574 & & 0,5 & 0,00518 & \\
\hline & & \multicolumn{2}{|c|}{$\alpha_{2}$} & & \multicolumn{2}{|c|}{$\alpha_{2}$} & & \multicolumn{2}{|c|}{$\alpha_{2}$} \\
\hline & $\alpha_{1}$ & 0,3 & 0,5 & $\alpha_{1}$ & 0,3 & 0,5 & $\alpha_{1}$ & 0,3 & 0,5 \\
\hline \multirow{2}{*}{$\operatorname{EQM}(\hat{\lambda})$} & 0,3 & 0,06259 & 0,2639 & 0,3 & 0,06736 & 0,32320 & 0,3 & 0,03359 & 0,06439 \\
\hline & 0,5 & 0,11008 & & 0,5 & 0,12668 & & 0,5 & 0,05182 & \\
\hline
\end{tabular}

Tabela 3.7: EQM das estimativas dos parâmetros do modelo INAR(2) $(\lambda=1)$ baseado em 500 réplicas e $N=200$.

Finalizando o estudo de simulação dos processos INAR, temos os resultados sumarizados de viés médio e EQM nas Tabelas 3.6 e 3.7, respectivamente. Como já mencionado em cada um dos processos simulados, o estimador de máxima verossimilhança tem resultados melhores, para as três combinações de parâmetros propostas. Entre os estimadores de Yule-Walker e mínimos quadrados condicionais não houve uma diferença tão expressiva quando comparado com máxima verossimilhança, mas observa-se que YW tem uma pequena vantagem em relação a MQC. 


\subsection{Modelos RCINAR}

Comumente os modelos considerados para séries temporais pertencem à classe de modelos lineares com coeficientes constantes. Em muitas situações esta classe não consegue ser a melhor proposta para séries temporais encontradas no mercado, pois existem casos em que o parâmetro pode ser variante no tempo. No campo da medicina temos como exemplo o número de pacientes doentes $X_{t}$ no $t$-ésimo mês. O modelo INAR(1) com $\alpha \circ X_{t-1}$ pacientes remanescentes do mês anterior pode descrever esse problema, no entanto, a taxa $\alpha$ pode ser não determinística ou ser função de fatores externos, como a qualidade do cuidado com pacientes ou a presença de um vírus causando epidemia, entre outros. Para diferenciar dos modelos INAR, utiliza-se $\phi_{t}$ no lugar de $\alpha$ como o coeficiente do modelo, variante em t.

A investigação da classe de modelos com coeficientes aleatórios iniciou-se no mercado com o estudo de Kendall (1953), onde foi ajustado um modelo autorregressivo de segunda ordem a séries econômicas em que os coeficientes mudavam suavemente com o passar do tempo de acordo com as mudanças da economia. O estudo por um tipo de modelo, que considerasse flutuações do coeficiente ao decorrer do tempo, prosperou com a pesquisa de Garbade (1977) que considerou o processo $\phi_{t}$ como um passeio aleatório simples. Essas ideias ganharam força e tiveram abordagem no campo dos modelos discretos; Zheng et al. (2007) introduziram essa metodologia aos modelos INAR. O coeficiente $\alpha$ foi substituído por um $\phi_{t}$, realização de uma variável aleatória i.i.d. com domínio em $[0,1)$. Esse tipo de modelo surgiu como uma extensão do modelo INAR, sendo chamado de RCINAR (random integer-valued autoregressive process). Análogo à representação dada em (3.1), pode-se definir o modelo RCINAR da seguinte forma:

$$
X_{t}=C_{t-1}+I_{t}
$$

em que a parte $C_{t-1}$ agora depende do histórico da série mas também de um coeficiente aleatório.

Os modelos RCINAR e INAR possuem representações muito parecidas, assim como suas propriedades, no entanto os modelos RCINAR exibem um conceito de distribuição para o parâmetro $\alpha$ do modelo INAR. Nesta sessão será apresentado o modelo RCINAR de ordem $p$, suas propriedades e métodos de estimação.

\subsubsection{Modelo RCINAR(p)}

O modelo RCINAR de ordem $p$, introduzido por Zheng et al. (2006) é definido como:

$$
X_{t}=\sum_{j=1}^{p} \phi_{j}^{(t)} \circ X_{t-j}+I_{t},
$$

em que $I_{t}$ é uma sequência aleatória i.i.d. inteira não negativa tal que $E\left(I_{t}^{4}\right) \leq \infty ; \phi_{j}^{(t)}$ é uma sequência aleatória i.i.d. e independente de $I_{t}$, com domínio em $[0,1)$.

Considerando

$$
\Phi_{j}=E\left(\phi_{j}^{(t)}\right), \sigma_{\phi_{i}}^{2}=\operatorname{var}\left(\phi_{i}^{(t)}\right), \sigma_{i j}=\operatorname{cov}\left(\phi_{i}^{(t)}, \phi_{j}^{(t)}\right), \lambda=E\left(I_{t}\right) \text { e } \sigma_{I}^{2}=\operatorname{var}\left(I_{t}\right),
$$

pode-se demonstrar que: 
- $E\left[X_{t} \mid X_{t-j}, 1 \leq j \leq p\right]=\sum_{j=1}^{p} \Phi_{j} X_{t-j}+\lambda$

- $E\left[X_{t}\right]=\frac{\lambda}{\left(1-\sum_{j=1}^{p} \Phi_{j}\right)}$ se $\sum_{j=1}^{p} \Phi_{j}<1$

- $\operatorname{Var}\left[X_{t} \mid X_{t-j}, \phi_{j}^{(t)}, 1 \leq j \leq p\right]=\sum_{j=1}^{p} \phi_{j}^{(t)}\left(1-\phi_{j}^{(t)}\right) X_{t-j}+\sigma_{I}^{2}$

- $\operatorname{Var}\left[X_{t} \mid X_{t-j}, 1 \leq j \leq p\right]=\sum_{j=1}^{p}\left(\sigma_{\Phi_{j}}^{2} X_{t-j}^{2}+\left(\Phi_{j}\left(1-\Phi_{j}\right)-\sigma_{\Phi_{j}}^{2}\right) X_{t-j}\right)+2$

$\times \sum_{1 \leq j \leq k \leq p} \sigma_{j k} X_{t-j} X_{t-k}+\sigma_{I}^{2}$

- $\gamma(k)=\sum_{j=1}^{p} \Phi_{j} \gamma(k-j)$

Resultado 3.2.1. Suponha $\sum_{j=1}^{p} \Phi_{j}<1$ e o máximo absoluto autovalor de $E\left[A_{1}^{T} \otimes A_{1}\right]<1$, em que

$$
A_{t}=\left[\begin{array}{ccccc}
\phi_{1}^{(t)} & \phi_{2}^{(t)} & \cdots & \phi_{p-1}^{(t)} & \phi_{p}^{(t)} \\
1 & 0 & \cdots & 0 & 0 \\
\vdots & \vdots & \ddots & \vdots & \vdots \\
0 & 0 & \cdots & 1 & 0
\end{array}\right]
$$

então existe uma única série estacionária assumindo valores inteiros, que satisfaça (3.22).

Mais detalhes desse resultado pode ser visto em Zheng et al. (2006), assim como a prova das propriedades. Como nos modelos INAR, a autocorrelação e autocorrelação parcial carregam informações de comportamento do modelo RCINAR. Serão mostradas autocorrelações amostrais desse modelo na Sessão 3.2.3.

\subsubsection{Métodos de estimação}

Assim como no modelo INAR, existe mais de uma metodologia de estimação para o modelo RCINAR. Nos últimos anos notou-se um crescente interesse por um estimador robusto para os parâmetros do modelo RCINAR; nesta sessão serão apresentados os métodos sugeridos por Zheng et al. (2006):

- Estimador de Mínimos Quadrados Condicionais

- Estimador de Máxima Verossimilhança

- Estimador de Quase-Verossimilhança Modificada 


\section{Mínimos Quadrados Condicionais (MQC)}

Seja o vetor de parâmetros dado por $\beta=\left(\Phi_{j}, 1 \leq j \leq p, \lambda\right)^{T}$ e a quantidade

$$
S(\beta)=\sum_{t=p+1}^{n}\left(X_{t}-\sum_{j=1}^{p} \Phi_{j} X_{t-j}-\lambda\right)^{2}
$$

O estimador de mínimos quadrados condicionais (MQC) de $\beta$ é obtido minimizando a quantidade $S(\beta)$, e é dado por:

$$
\hat{\beta}=Q^{-1} q,
$$

em que

$$
q=\left(\sum_{t=p+1}^{n} X_{t} X_{t-1}, \cdots, \sum_{t=p+1}^{n} X_{t} X_{t-p}, \sum_{t=p+1}^{n} X_{t}\right)^{T}
$$

e

$$
Q=\left[\begin{array}{ccccc}
\sum_{t=p+1}^{n} X_{t-1}^{2} & \sum_{t=p+1}^{n} X_{t-1} X_{t-2} & \cdots & \sum_{t=p+1}^{n} X_{t-1} X_{t-p} & \sum_{t=p+1}^{n} X_{t-1} \\
\sum_{t=p+1}^{n} X_{t-2} X_{t-1} & \sum_{t=p+1}^{n} X_{t-2}^{2} & \cdots & \sum_{t=p+1}^{n} X_{t-2} X_{t-p} & \sum_{t=p+1}^{n} X_{t-2} \\
\vdots & \vdots & \ddots & \vdots & \vdots \\
\sum_{t=p+1}^{n} X_{t-1} & \sum_{t=p+1}^{n} X_{t-2} & \cdots & \sum_{t=p+1}^{n} X_{t-p} & n-p
\end{array}\right] .
$$

Resultado 3.2.2. A distribuição assintótica do estimador de $M Q C$ é dada por

$$
\sqrt{n}(\hat{\beta}-\beta) \stackrel{d}{\rightarrow} N\left(\mathbf{0}, \mathbf{V}^{-\mathbf{1}} \mathbf{W} \mathbf{V}^{-\mathbf{1}}\right)
$$

em que $V=\lim _{n \rightarrow \infty} \frac{1}{n} Q$ e $W=\left(w_{i j}\right)_{(p+1) \times(p+1)}$,

$$
\begin{aligned}
w_{i j} & =E\left[X_{p+1-i} X_{p+1-j} \operatorname{Var}\left(X_{p+1} \mid X_{1}, \cdots, X_{p}\right)\right], 1 \leq i, j \leq p ; \\
w_{i(p+1)} & =E\left[X_{p+1-i} \operatorname{Var}\left(X_{p+1} \mid X_{1}, \cdots, X_{p}\right)\right], 1 \leq i \leq p ; \\
w_{(p+1)(p+1)} & =E\left[\operatorname{Var}\left(X_{p+1} \mid X_{1}, \cdots, X_{p}\right)\right] .
\end{aligned}
$$

Tal resultado é provado em Klimko e Nelson (1978). 


\section{Máxima Verossimilhança (MV)}

Para obtenção da verossimilhança utiliza-se a propriedade de Markov. Dado o modelo proposto em (3.22) pode-se calcular a probabilidade em cada instante do tempo, como segue:

$$
\begin{aligned}
P\left(X_{t}=i \mid X_{t-1}=i_{1}, \cdots, X_{t-p}=i_{p}\right) & =\sum_{k=0}^{m i n\left(i, \sum_{j=1}^{p} i_{j}\right)} f_{z}(i-k) \prod_{0 \leq \sum_{j=1}^{p} k_{j} \leq k}\left(\begin{array}{c}
i_{j} \\
k_{j}
\end{array}\right) \\
& \times \int_{0 \leq \phi_{1}^{(t)} \ldots \phi_{p}^{(t)} \leq 1} \prod_{j=1}^{p}\left[\phi_{j}^{(t)}\right]^{k_{j}}\left[1-\phi_{1}^{(t)}\right]^{i_{j}-k_{j}} d P_{\phi_{1}^{(t)} \ldots \phi_{p}^{(t)}},
\end{aligned}
$$

em que $P_{\phi_{1}^{(t)} \ldots \phi_{p}^{(t)}}$ é a distribuição conjunta de $\left(\phi_{1}^{(t)}, \cdots, \phi_{p}^{(t)}\right)$.

Utilizando a probabilidade dada por (3.27), pode-se chegar a verossimilhança

$$
L(\eta)=P\left(X_{p}=i_{1}^{p+1}, \cdots, X_{1}=i_{p}^{p+1}\right) \prod_{t=p+1}^{n} P\left(X_{t}=i^{(t)} \mid X_{t-1}=i_{1}^{(t)}, \cdots, X_{t-p}=i_{p}^{(t)}\right),
$$

considerando $\eta$ o vetor de parâmetros $\left(\phi_{1}^{(t)}, \cdots, \phi_{p}^{(t)}, \lambda\right)$.

O estimador de máxima verossimilhança $\hat{\eta}$ é o valor que maximiza a função de verossimilhança (3.28). O cálculo da probabilidade dada em (3.27) pode ser bastante complicado computacionalmente, para contornar esse fato introduz-se o estimador de quase verossimilhança modificada.

\section{Quase-Verossimilhança Modificada (QVM)}

Considere o modelo apresentado em (3.22), e sejam

$$
\theta=\left(\sigma_{\phi_{j}}^{2}, \eta_{j}, \sigma_{k j}, 1 \leq j \leq p, 1 \leq k<j \leq p, \sigma_{I}^{2}\right),
$$

em que

$$
\sigma_{\phi_{j}}^{2}=\operatorname{var}\left(\phi_{j}^{(t)}\right), \eta_{j}=\phi_{j}\left(1-\phi_{j}\right)-\sigma_{\phi_{j}}^{2} \text { e } \sigma_{I}^{2}=\operatorname{var}\left(I_{t}\right)
$$

e

$$
\beta=\left(\phi_{j}, 1 \leq j \leq p, \lambda\right)^{T} .
$$

Utilizando a variância condicional $V_{\theta}\left(X_{t} \mid X_{t-1}, \cdots, X_{t-p}\right):=\operatorname{Var}\left(X_{t} \mid X_{t-1}, \cdots, X_{t-p}\right)$, o conjunto de equações de estimação de quase-verossimilhança são:

$$
\begin{aligned}
\sum_{t=p+1}^{n} V_{\theta}^{-1}\left(X_{t} \mid X_{t-1}, \cdots, X_{t-p}\right) X_{t-1}\left(X_{t}-\sum_{j=1}^{p} \phi_{j} X_{t-j}-\lambda\right) & =0 \\
& \vdots \\
\sum_{t=p+1}^{n} V_{\theta}^{-1}\left(X_{t} \mid X_{t-1}, \cdots, X_{t-p}\right) X_{t-p}\left(X_{t}-\sum_{j=1}^{p} \phi_{j} X_{t-j}-\lambda\right) & =0 \\
\sum_{t=p+1}^{n} V_{\theta}^{-1}\left(X_{t} \mid X_{t-1}, \cdots, X_{t-p}\right)\left(X_{t}-\sum_{j=1}^{p} \phi_{j} X_{t-j}-\lambda\right) & =0
\end{aligned}
$$


Seja $\tilde{\beta}$ a solução das equações de estimação (3.29), considerando que $\theta$ é conhecido. Zheng et al. (2006) propõem um estimador de quase-verossimilhança para $\beta$ quando $\theta$ não é conhecido. A estimação é realizada em duas fases, primeiro se estima $\theta$ através de um método consistente, em seguida a estimativa de $\theta$ é usada para resolver as equações (3.29) obtendo-se estimativas de quaseverossimilhança para $\beta$.

O estimador para $\theta$ explorado, denominado Method II, foi proposto por Zheng et al. (2006) e se baseia em mínimos quadrados condicionais.

Seja

$$
\begin{aligned}
S= & \sum_{t=p+1}^{n}\left[\left(X_{t}-\sum_{i=1}^{p} \hat{\Phi}_{i} X_{t-1}-\hat{\lambda}\right)^{2}\right. \\
& \left.-\sum_{i=1}^{p}\left(\sigma_{\phi_{i}}^{2} X_{t-i}^{2}+\left(\hat{\Phi}_{i}\left(1-\hat{\Phi}_{i}\right)-\sigma_{\Phi_{i}}^{2}\right) X_{t-i}\right)-2 \sum_{1<i<j<p} \sigma_{i j} X_{t-i} X_{t-j}-\sigma_{I}^{2}\right]^{2}
\end{aligned}
$$

A estimação de $\theta$ é obtida pela minimização de (3.30). O cálculo de $S$ depende de estimadores consistentes para $\Phi_{i}$ e $\lambda$, que são obtidos utilizando estimadores de MQC. Os estimadores dados não garantem estimativas sempre positivas para as variâncias de $\phi$ e $Z$ para pequenas amostras; nesse caso, Zheng et al. (2006) propõem substituir a estimativa da variância, quando negativa, por uma estimativa dada pelo estimador de máxima verossimilhança.

Resultado 3.2.3. A propriedade assintótica do estimador de quase verossimilhança modificada é dada por.

$$
\begin{aligned}
\sqrt{n}(\widetilde{\beta}-\beta) \stackrel{d}{\rightarrow} N\left(\mathbf{0}, \mathbf{T}^{-\mathbf{1}}\right), \text { em que } \mathbf{T}=\left(t_{i j}\right)_{(p+1) \times(p+1)} \\
t_{i j}=E\left[X_{p+1-i} X_{p+1-j} V_{\theta}^{-1}\left(X_{p+1} \mid X_{p}, \cdots, X_{1}\right)\right], 1 \leq i, j \leq p ; \\
t_{i(p+1)}=E\left[X_{p+1-i} V_{\theta}^{-1}\left(X_{p+1} \mid X_{p}, \cdots, X_{1}\right)\right], 1 \leq i \leq p ; \\
t_{(p+1)(p+1)}=E\left[V_{\theta}^{-1}\left(X_{p+1} \mid X_{p}, \cdots, X_{1}\right)\right], 1 \leq i \leq p .
\end{aligned}
$$

O Resultado 3.2.3 não é afetado quando usa-se um estimador consistente para $\theta$. Mais detalhes são apresentados em Zheng et al. (2006).

\subsubsection{Estudo de simulação}

A sessão dos modelos RCINAR é concluída com o estudo de simulação. Assim como na Seção 3.1.4, esse estudo é usado para avaliar a eficiência dos estimadores.

O processo gerado possui a estrutura mostrada em (3.22), tratando $I_{t}$ como um processo de Poisson com média $\lambda=1$. Consideramos o parâmetro da probabilidade $\phi_{j}$ como uma variável aleatória com distribuição Beta. Os parâmetros utilizados na simulação são apresentados na Tabela 3.8 , em que $\sigma_{\phi_{j}}^{2}=0,05, j=1,2$. 


\begin{tabular}{cccc}
\hline \hline Modelo & $\mathrm{N}$ & $E\left[\phi_{1}\right]$ & $E\left[\phi_{2}\right]$ \\
\hline \hline $\operatorname{RCINAR}(1)$ & 100 & 0,3 & - \\
$\operatorname{RCINAR}(1)$ & 100 & 0,5 & - \\
$\operatorname{RCINAR}(1)$ & 100 & 0,7 & - \\
\hline $\operatorname{RCINAR}(1)$ & 200 & 0,3 & - \\
$\operatorname{RCINAR}(1)$ & 200 & 0,5 & - \\
$\operatorname{RCINAR}(1)$ & 200 & 0,7 & - \\
\hline $\operatorname{RCINAR}(1)$ & 500 & 0,3 & - \\
$\operatorname{RCINAR}(1)$ & 500 & 0,5 & - \\
$\operatorname{RCINAR}(1)$ & 500 & 0,7 & - \\
\hline $\operatorname{RCINAR}(2)$ & 200 & 0,3 & 0,3 \\
$\operatorname{RCINAR}(2)$ & 200 & 0,3 & 0,5 \\
$\operatorname{RCINAR}(2)$ & 200 & 0,5 & 0,3 \\
\hline \hline
\end{tabular}

Tabela 3.8: Modelos gerados - RCINAR.
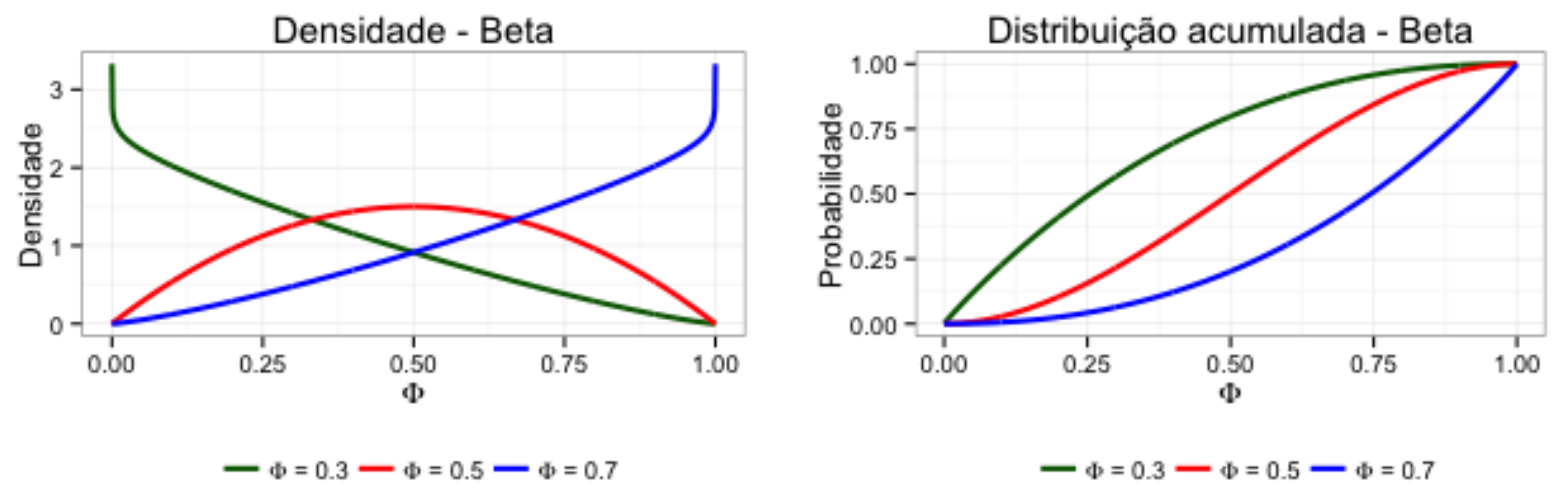

Figura 3.52: Distribuição Beta considerada para o estudo.

O estudo de simulação segue a sequência:

- Gera-se uma trajetória de um modelo definido.

- Estima-se os parâmetros com estimadores MQC e QVM.

- Armazenam-se as estimativas de $\phi_{j}$ e $\lambda$.

Diferente da simulação dos modelos INAR, o modelo RCINAR depende de um parâmetro aleatório em cada instante de tempo. Os valores desse parâmetro foram gerados independentes através de uma distribuição Beta. Cada linha da Tabela 3.8 é replicada 500 vezes, isso nos garante uma melhor avaliação dos estimadores. A análise das simulações está organizada da seguinte forma: 1) Visualização do histograma dos valores de $\phi_{j}, j=1,2$ de uma das réplicas e autocorrelação serial; 2) Um exemplo de trajetória gerada; 3) Histograma e boxplot de uma trajetória gerada; 4) Boxplot de cada parâmetro comparando as estimativas dadas por cada estimador proposto; 5) Histograma suavisado do viés das estimativas dadas por cada método de estimação. Ao final é exibido uma tabela resumo dos vieses e EQM. 
Processo RCINAR(1): $X_{t}=\phi_{t} \circ X_{t-1}, E\left[\phi_{t}\right]=0,3$.

Primeiro avalia-se o comportamento temporal do coeficiente $\phi_{t}$ gerado. Na Figura 3.53 à esquerda, temos o histograma dos valores de uma réplica gerada, nota-se o comportamento da distribuição Beta, como esperado, e à direita está o gráfico de autocorrelação, mostrando que os valores gerados são não correlacionados serialmente.

(a)
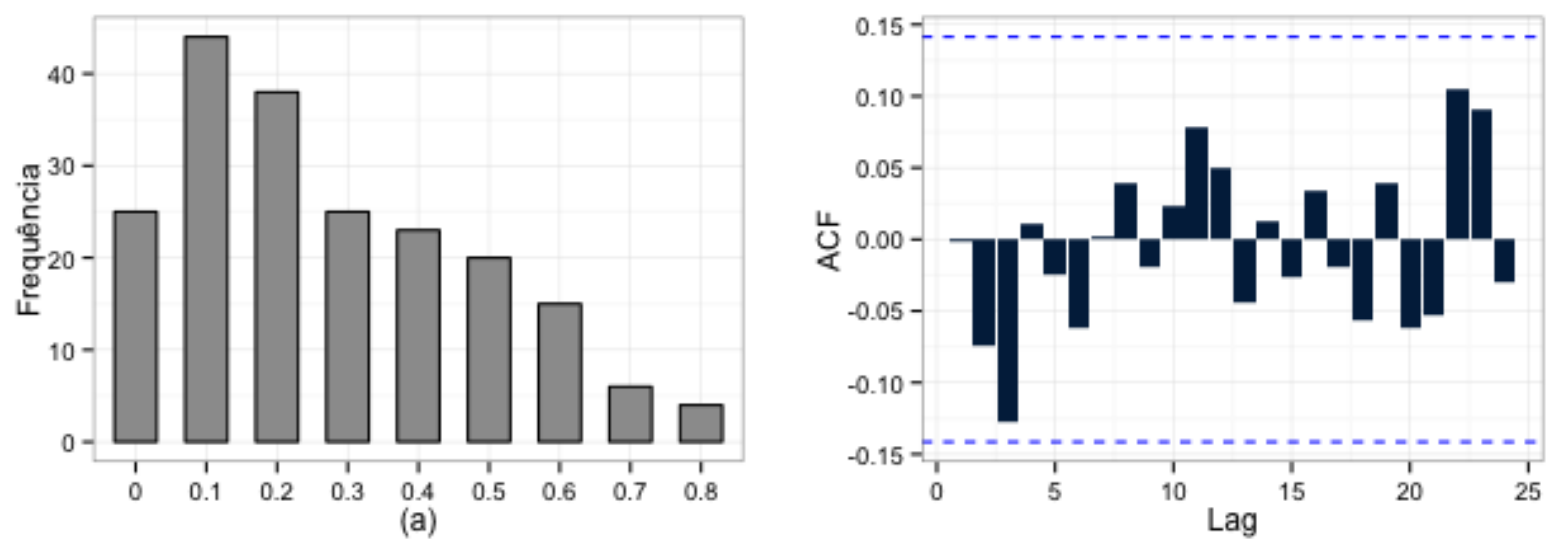

Figura 3.53: Histograma e ACF do $\phi_{t}$ simulado, RCINAR(1).

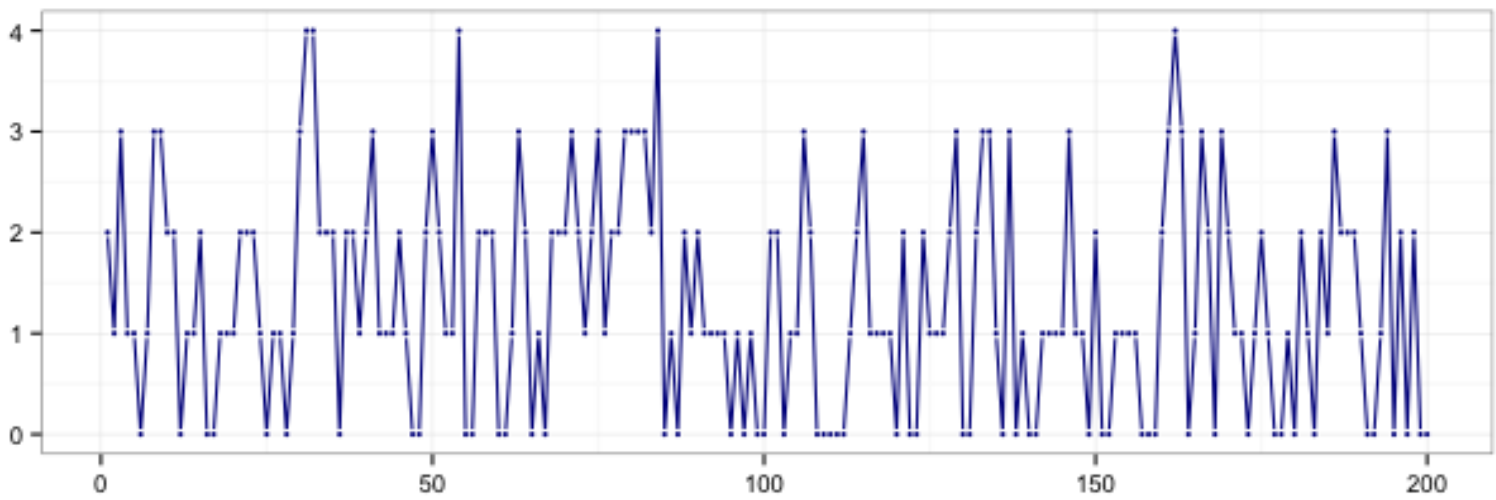

Figura 3.54: Gráfico de uma série simulada, $\operatorname{RCINAR(1):~} E\left[\phi_{t}\right]=0,3$ e $\lambda=1$.

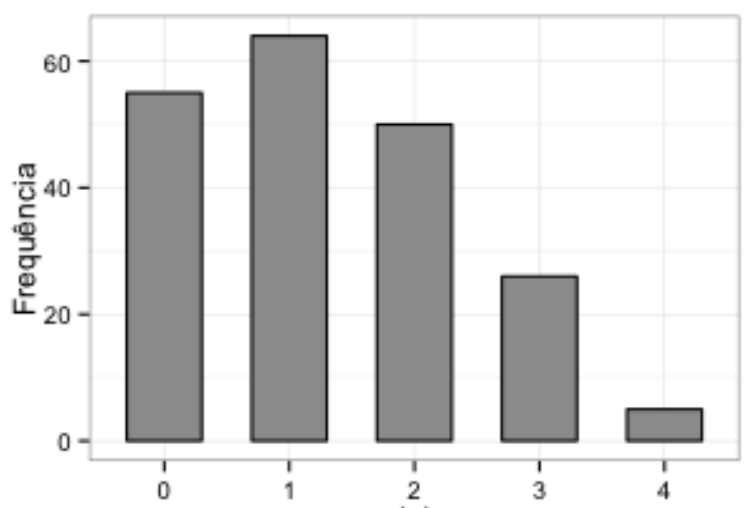

(a)

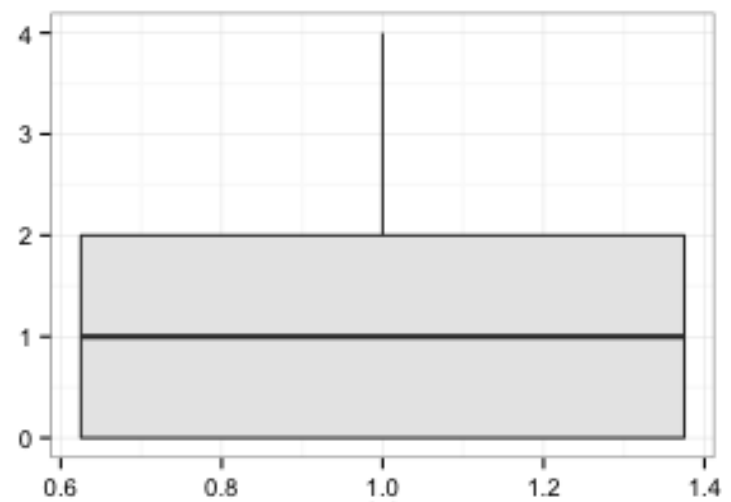

(b)

Figura 3.55: Histograma e boxplot da série simulada, $\operatorname{RCINAR(1):~} E\left[\phi_{t}\right]=0,3$ e $\lambda=1$. 

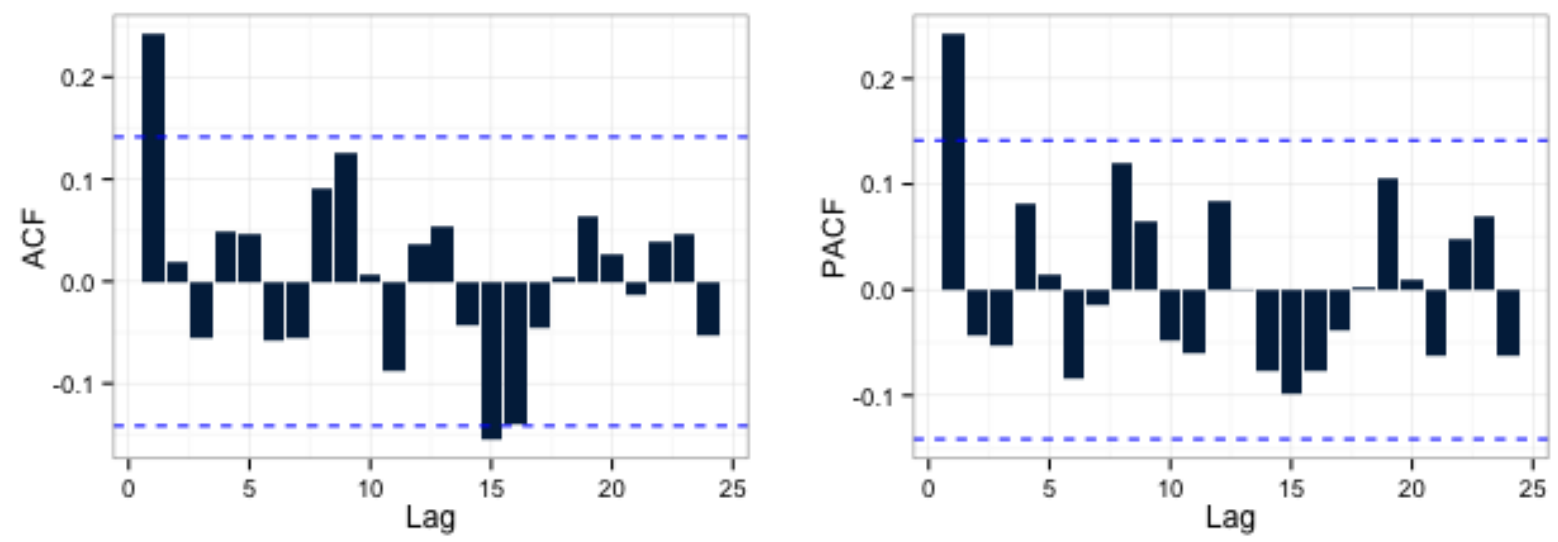

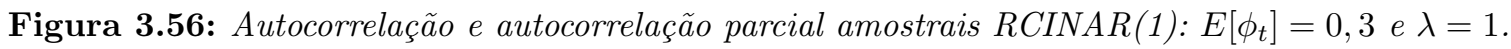

Nota-se que existem valores significantes de ordem um nos gráficos de ACF e PACF mostrados na Figura 3.56.

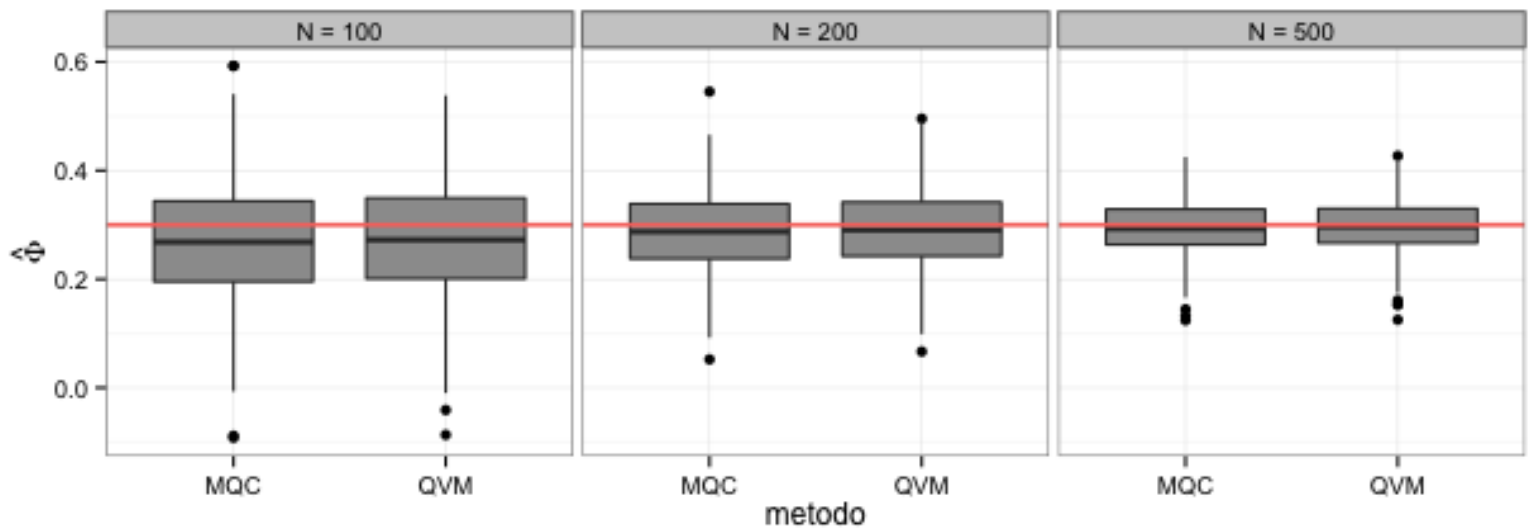

Figura 3.57: Boxplot das estimativas de $E\left[\phi_{t}\right]$ para o modelo gerado por $X_{t}=\phi_{t} \circ X_{t-1}+I_{t}$.

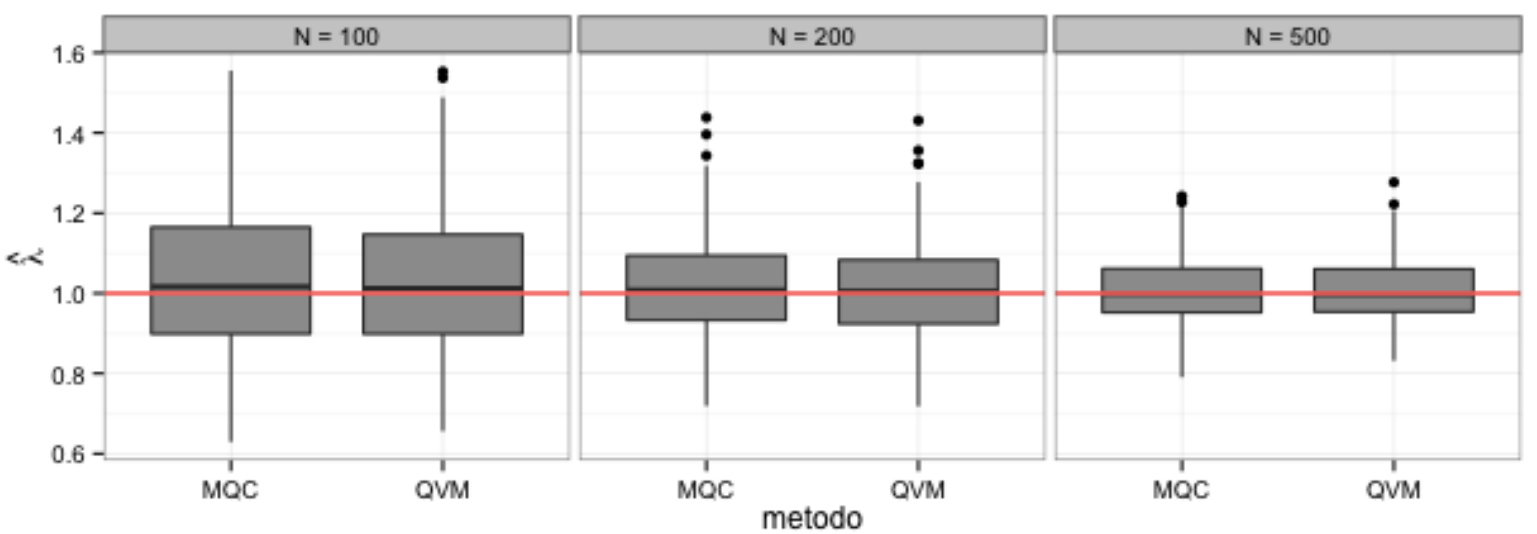

Figura 3.58: Boxplot das estimativas de $\lambda$ para o modelo gerado por $X_{t}=\phi_{t} \circ X_{t-1}+I_{t}$.

A Figura 3.57 apresenta o boxplot dos parâmetros estimados $\hat{\Phi}$, em que $\Phi=E\left[\phi_{t}\right]$. É fácil notar a redução da variabilidade dos valores estimados em ambos os métodos à medida que aumenta-se o tamanho da série. O que não é tão claro no boxplot é que o método de quase-verossimilhança modificada (QVM) têm suas estimativas mais próximas do verdadeiro valor. 
O mesmo pode-se notar na Figura 3.58, as estimativas se tornam mais concentradas a medida que aumenta-se o tamanho da série. Visualmente ambos os métodos têm suas estimativas bastante próximas do verdadeiro valor do parâmetro $\lambda$.

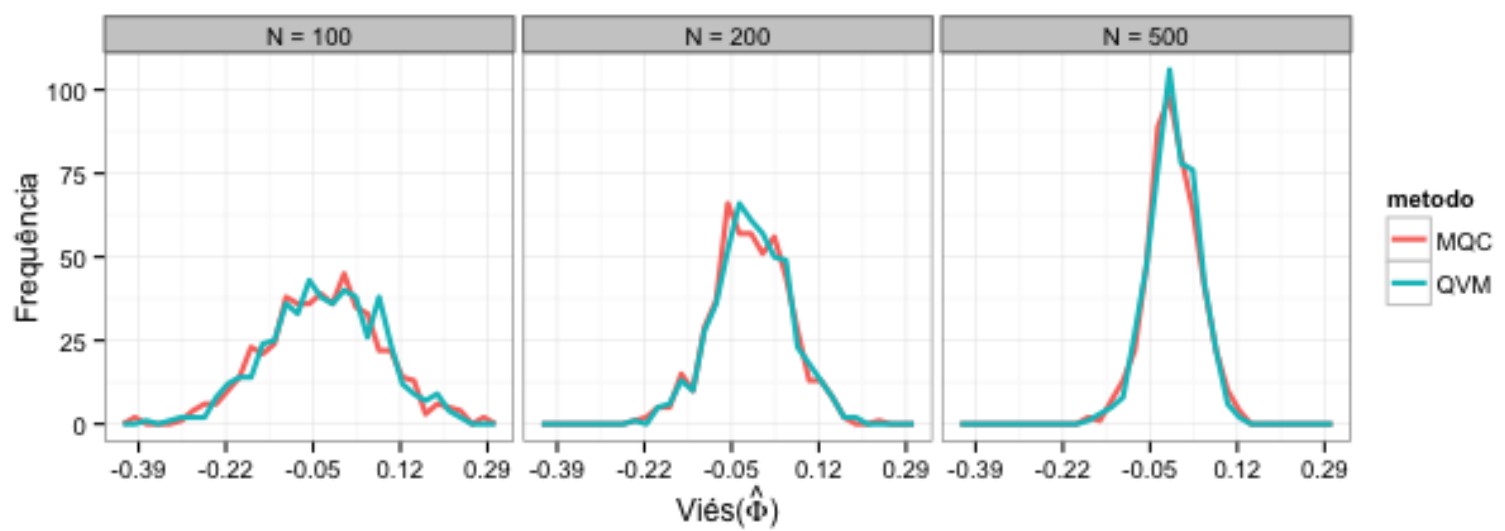

Figura 3.59: Histograma alisado do viés de $\hat{\Phi}$ para o modelo gerado por $X_{t}=\phi_{t} \circ X_{t-1}+I_{t}$.

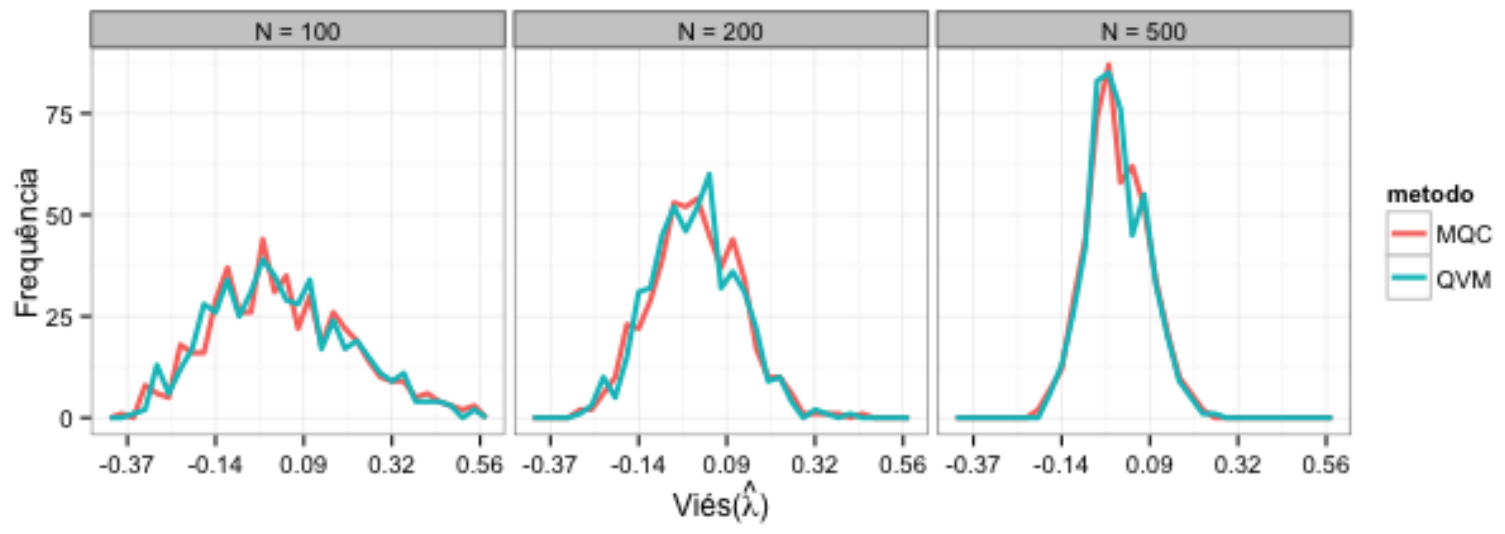

Figura 3.60: Histograma alisado do viés de $\hat{\lambda}$ para o modelo gerado por $X_{t}=\phi_{t} \circ X_{t-1}+I_{t}$.

A análise se encerra com os gráfico dos vieses; primeiro vemos na Figura 3.59 o histograma alisado dos vieses de $\hat{\Phi}$, dados pelos metodos MQC e QVM. Assim como na análise sobre os parâmetros estimados, o histograma alisado do viés não mostra muita diferença entre os métodos.

A Figura 3.60 reforça a afirmação acima: o viés está ao redor de zero para ambos estimadores e nenhum se destaca em relação ao outro. 

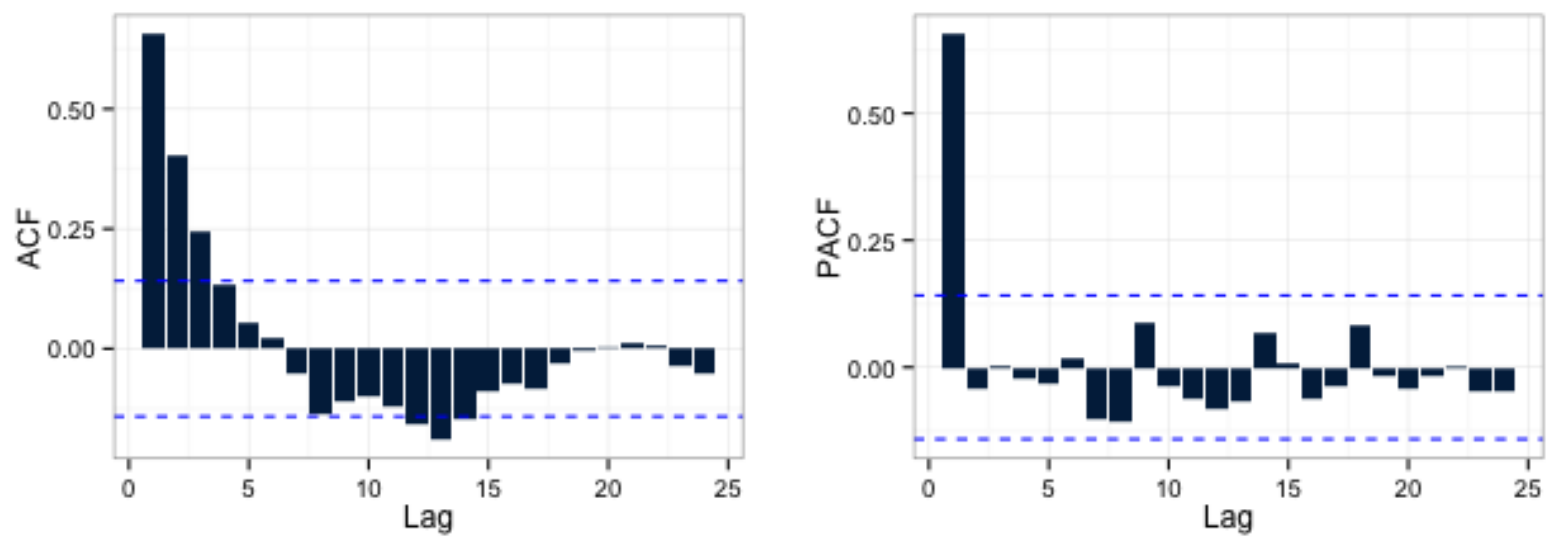

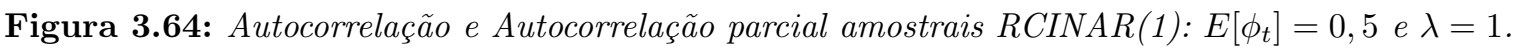

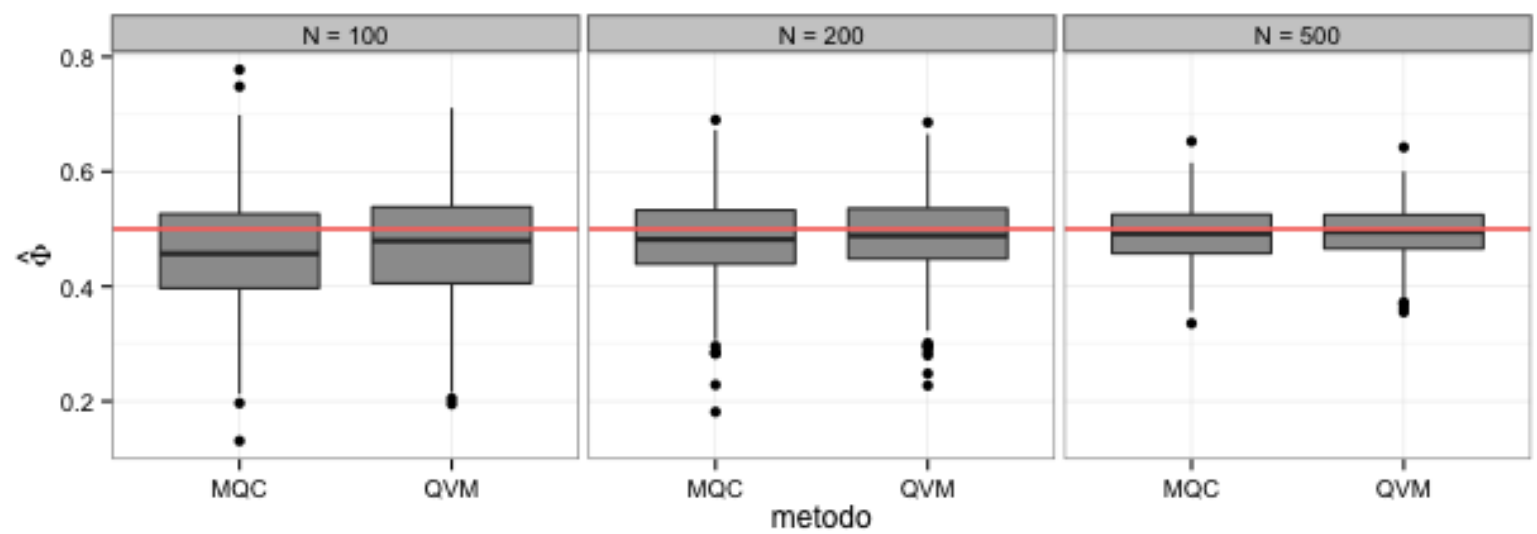

Figura 3.65: Boxplot das estimativas de $E\left[\phi_{t}\right]$ para o modelo gerado por $X_{t}=\phi_{t} \circ X_{t-1}+I_{t}$.

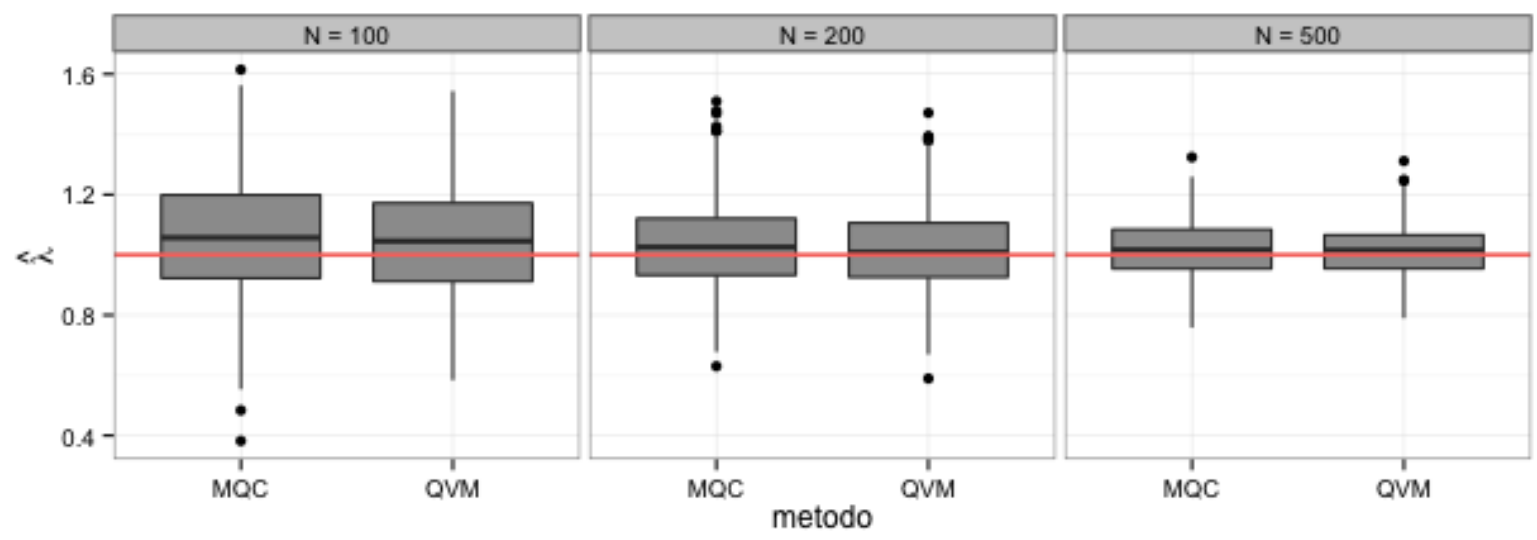

Figura 3.66: Boxplot das estimativas de $\lambda$ para o modelo gerado por $X_{t}=\phi_{t} \circ X_{t-1}+I_{t}$.

As estimativas dadas por QVM se mostram mais próximas do verdadeiro valor em média; essa afirmação é verificada através do boxplot das estimativas de $\Phi$ na Figura 3.65. A variância das estimativas dada por QVM e MQC são próximas e reduz conforme aumenta-se o tamanho da amostra.

As estimativas de $\lambda$ são exibidas na Figura 3.66; o boxplot mostra que as estimativas dadas por QVM e MQC têm valores próximos do verdadeiro valor. 
Processo RCINAR(1): $X_{t}=\phi_{t} \circ X_{t-1}, E\left[\phi_{t}\right]=0,5$.

A análise tem início com o comportamento do coeficiente $\phi_{t}$ gerado, mostrado na Figura 3.61. Em seguida temos uma trajetória do processo $X_{t}=\phi_{t} \circ X_{t-1}+I_{t}$, em que $E\left[\phi_{t}\right]=0,5$, na Figura 3.62. O histograma da trajetória é exibida na Figura 3.63 junto com seu boxplot; vemos que a média da trajetória está cerca de 2, valor esperado para o processo gerado com parâmetro $\lambda=1$ e $\Phi=0.5$.

A autocorrelação e autocorrelação parcial amostrais mostradas na Figura 3.64, exibem fortes características do processo RCINAR(1), em que a ACF decai exponencialmente e a PACF apresenta valor significante de ordem um.
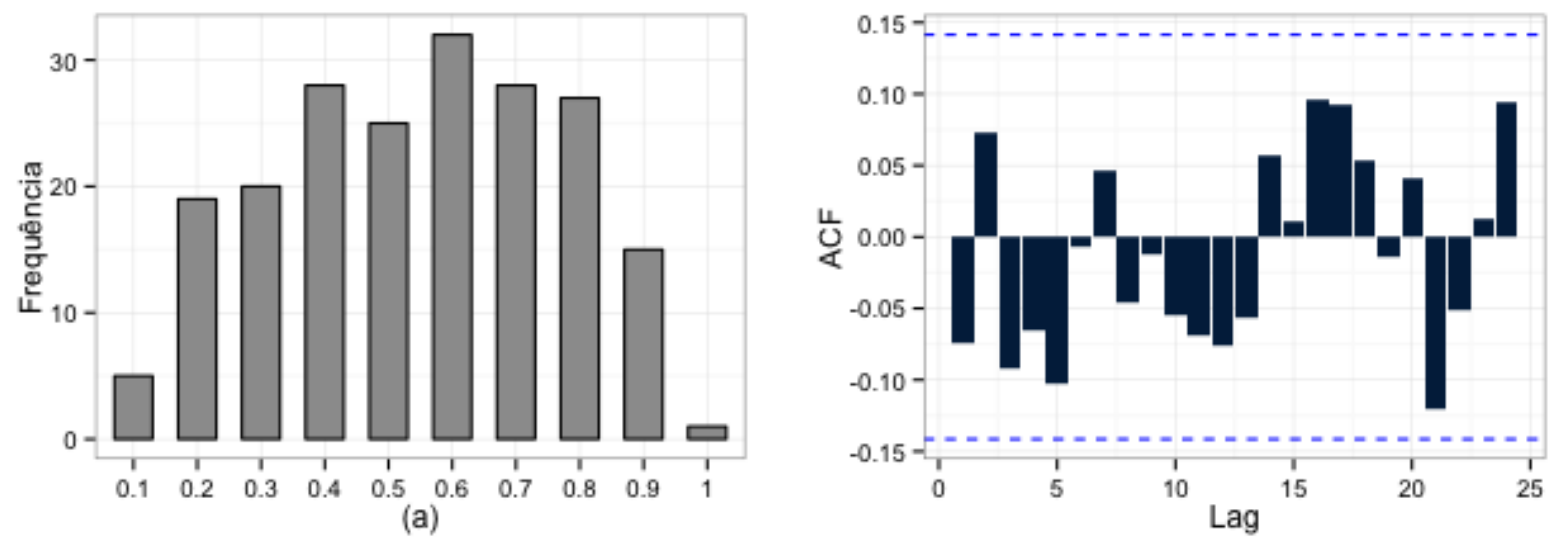

Figura 3.61: Histograma e ACF do $\phi_{t}$ simulado, RCINAR(1).

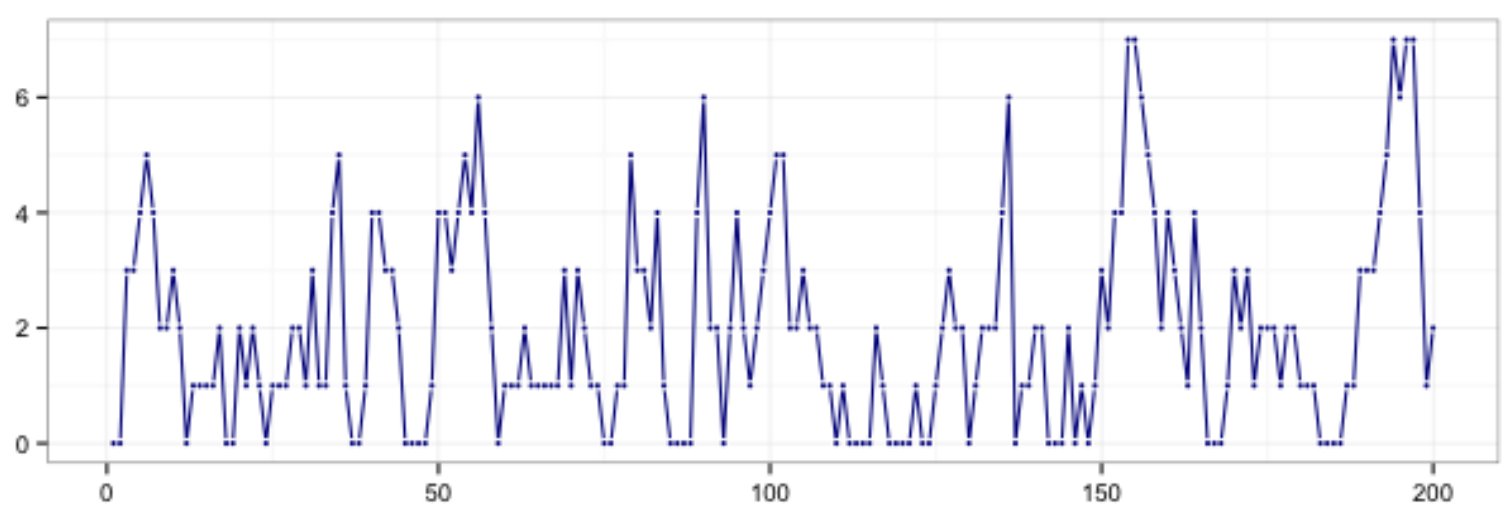

Figura 3.62: Gráfico de uma série simulada, $R C I N A R(1): E\left[\phi_{t}\right]=0,5$ e $\lambda=1$.

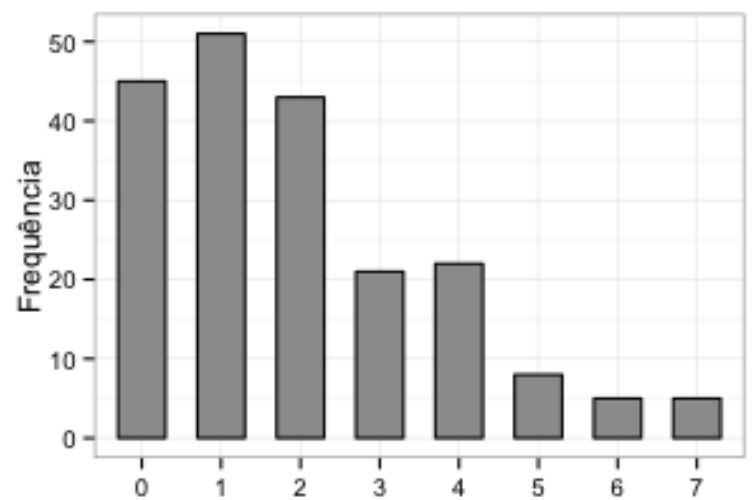

(a)

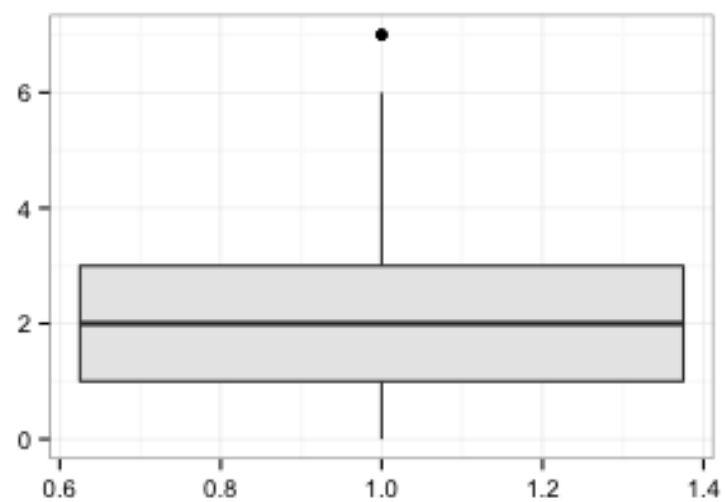

(b)

Figura 3.63: Histograma e boxplot da série simulada, $R C I N A R(1): E\left[\phi_{t}\right]=0,5$ e $\lambda=1$. 

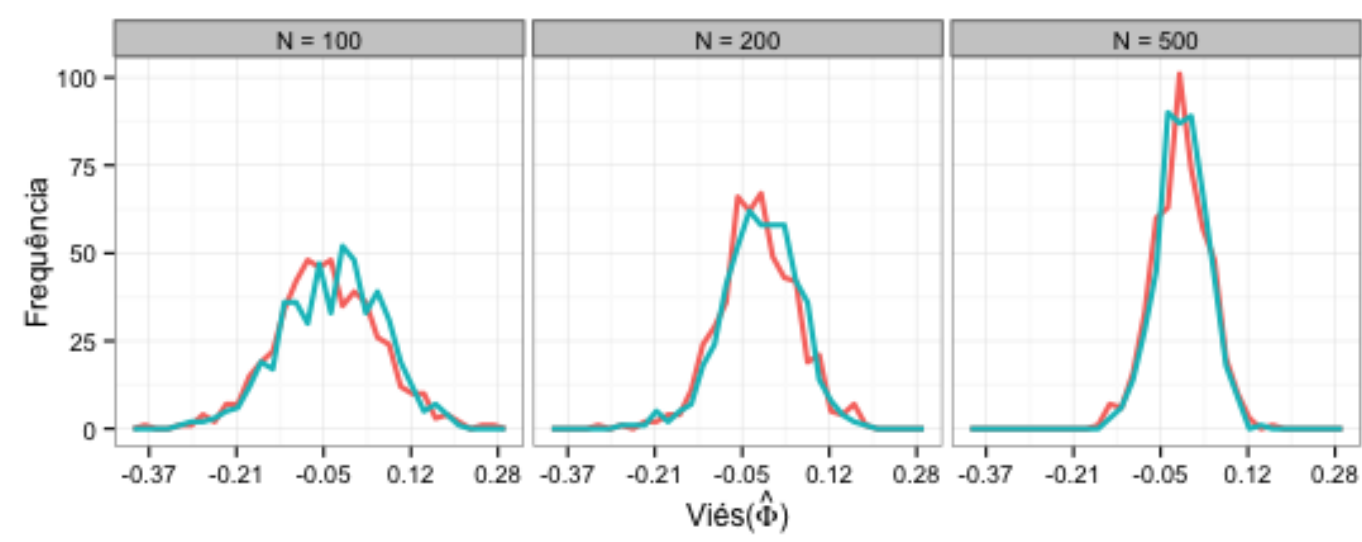

metodo

- MQC

- Qvm

Figura 3.67: Histograma alisado do viés de $\hat{\Phi}$ para o modelo gerado por $X_{t}=\phi_{t} \circ X_{t-1}+I_{t}$.

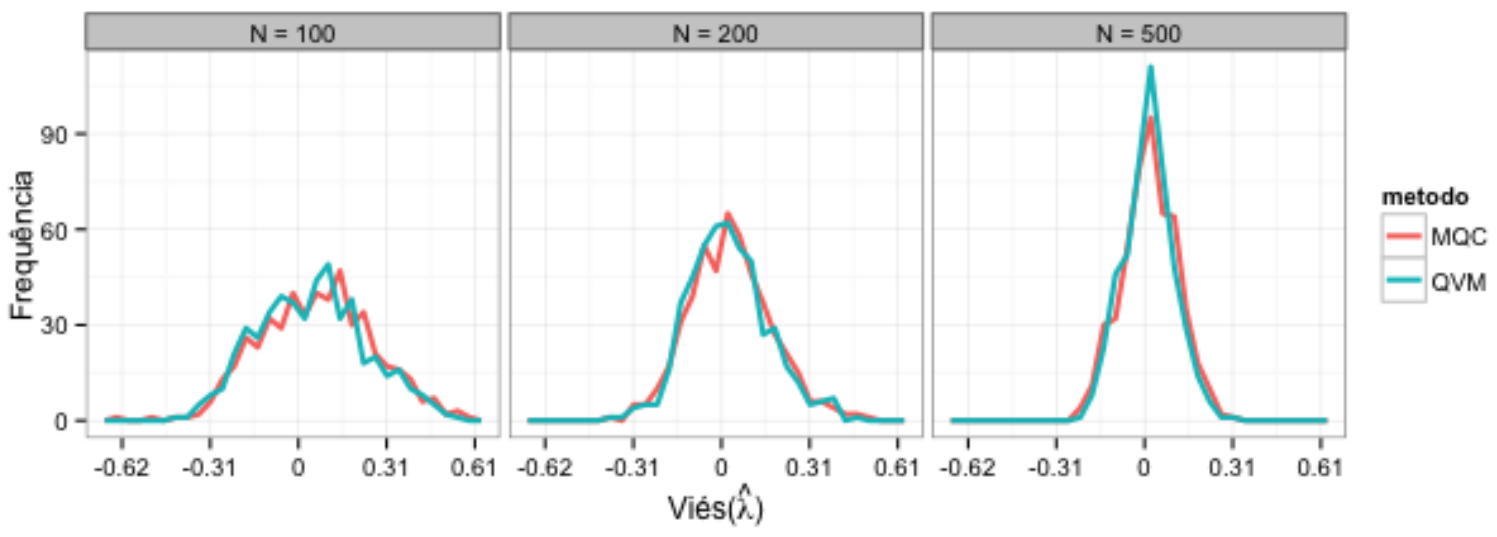

Figura 3.68: Histograma alisado do viés de $\hat{\lambda}$ para o modelo gerado por $X_{t}=\phi_{t} \circ X_{t-1}+I_{t}$.

As Figuras 3.67 e 3.68 mostram os histogramas alisados dos vieses de $\hat{\Phi}$ e $\hat{\lambda}$. Nota-se que as estimativas se concentram mais em torno de zero quando aumenta-se o tamanho da série. Não existe grande distinção entre os métodos de estimação. 
Processo RCINAR(1): $X_{t}=\phi_{t} \circ X_{t-1}, E\left[\phi_{t}\right]=0,7$.

Seguindo com o estudo das simulações, agora temos apresentados os resultados do processo RCINAR(1) com $\Phi=0,7$. A Figura 3.69 apresenta o histograma dos valores gerados de $\phi_{t}$ para uma réplica, seu comportamento se assemelha ao da distribuição Beta.

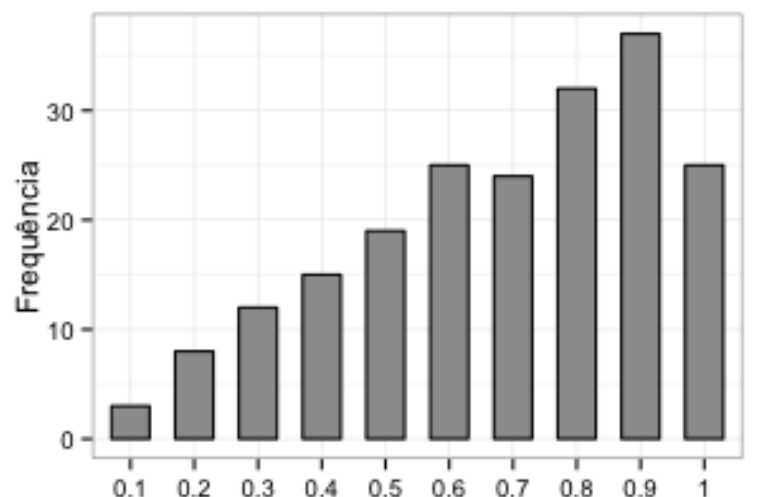

(a)

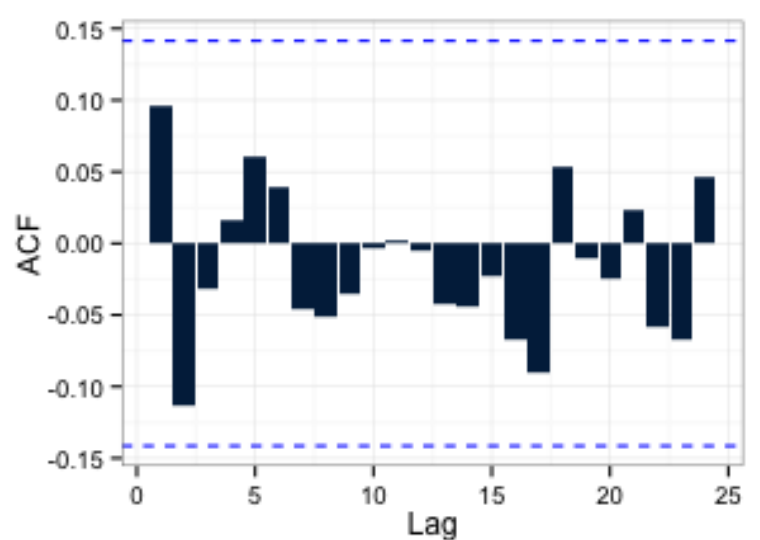

Figura 3.69: Histograma e ACF do $\phi_{t}$ simulado, RCINAR(1).

As Figuras 3.70 e 3.71 apresentam a trajetória, o histograma e boxplot de um processo RCINAR(1) simulado.

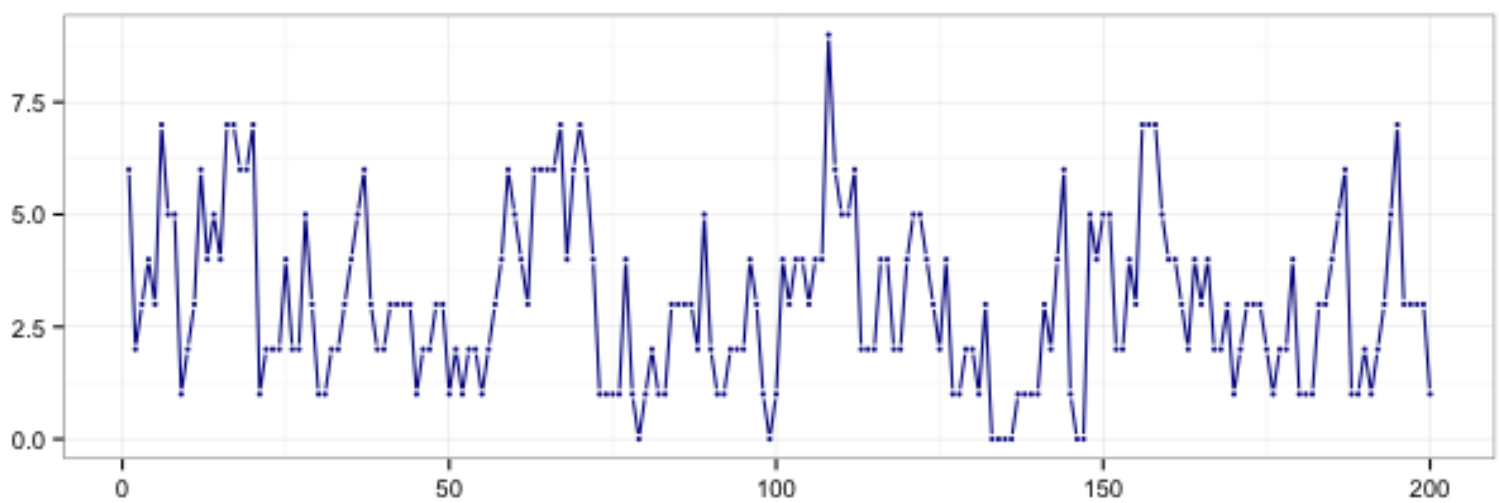

Figura 3.70: Gráfico de uma série simulada, $R \operatorname{CINAR(1):~} E\left[\phi_{t}\right]=0,7$ e $\lambda=1$.

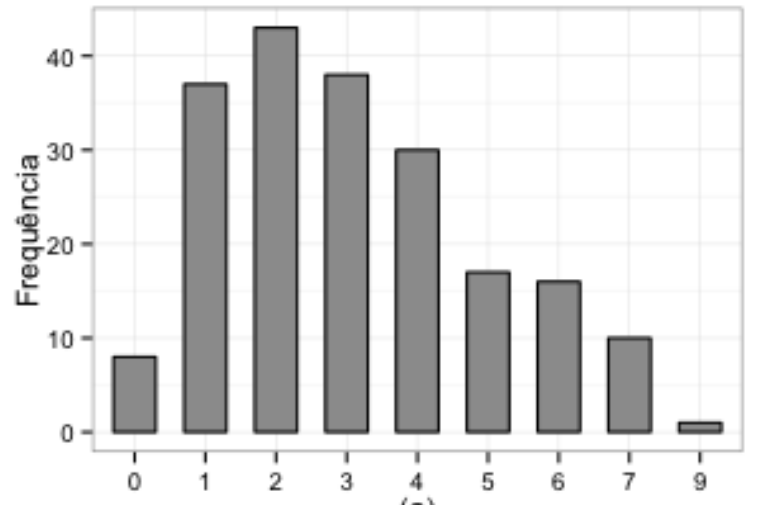

(a)

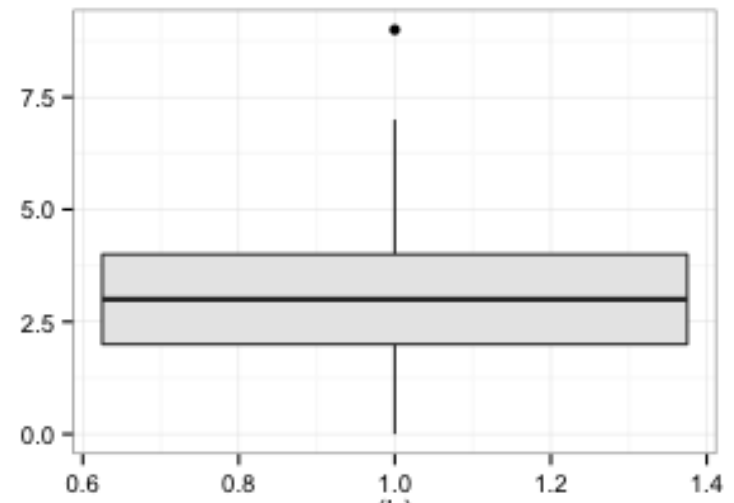

(b)

Figura 3.71: Histograma e boxplot da série simulada, $R \operatorname{CINAR(1):~} E\left[\phi_{t}\right]=0,7$ e $\lambda=1$. 
O comportamento da ACF e PACF apresentado na Figura 3.72 exibe a característica do processo RCINAR(1), com ordem um significante. O boxplot das estimativas de $\hat{\Phi}$ e $\hat{\lambda}$, dadas por MQC e QVM são apresentados nas Figuras 3.73 e 3.74 .
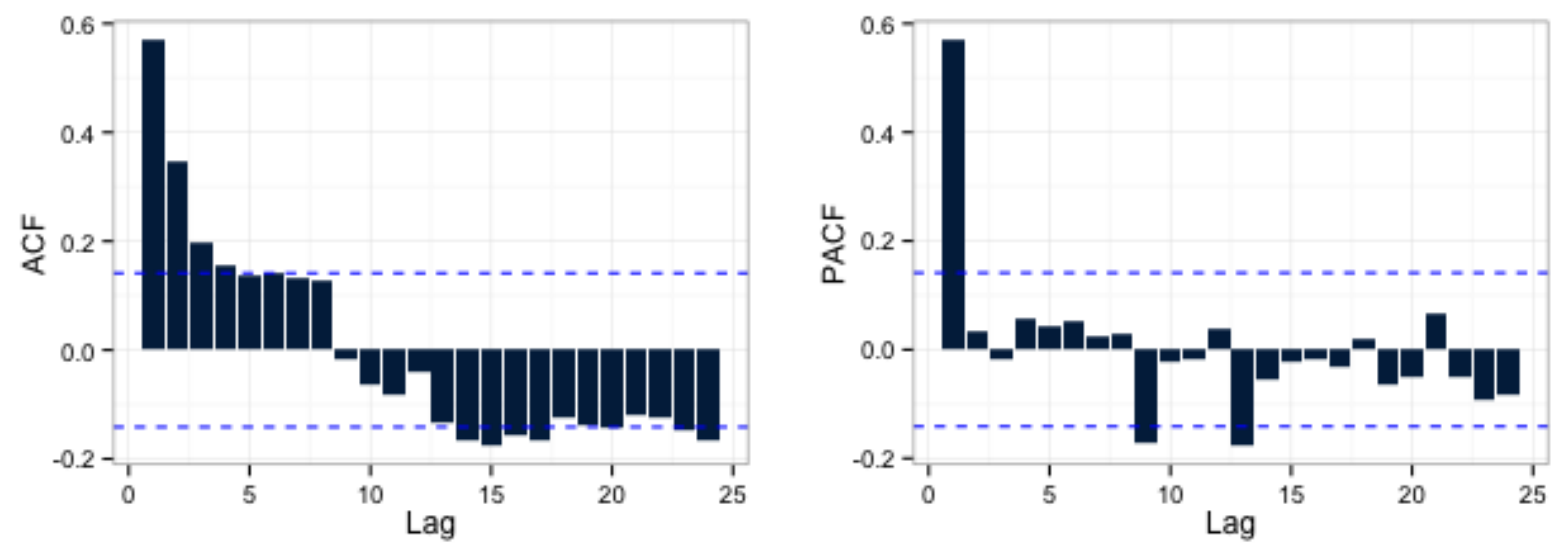

Figura 3.72: Autocorrelação e Autocorrelação parcial amostrais $\operatorname{RCINAR(1):~} E\left[\phi_{t}\right]=0,7$ e $\lambda=1$.

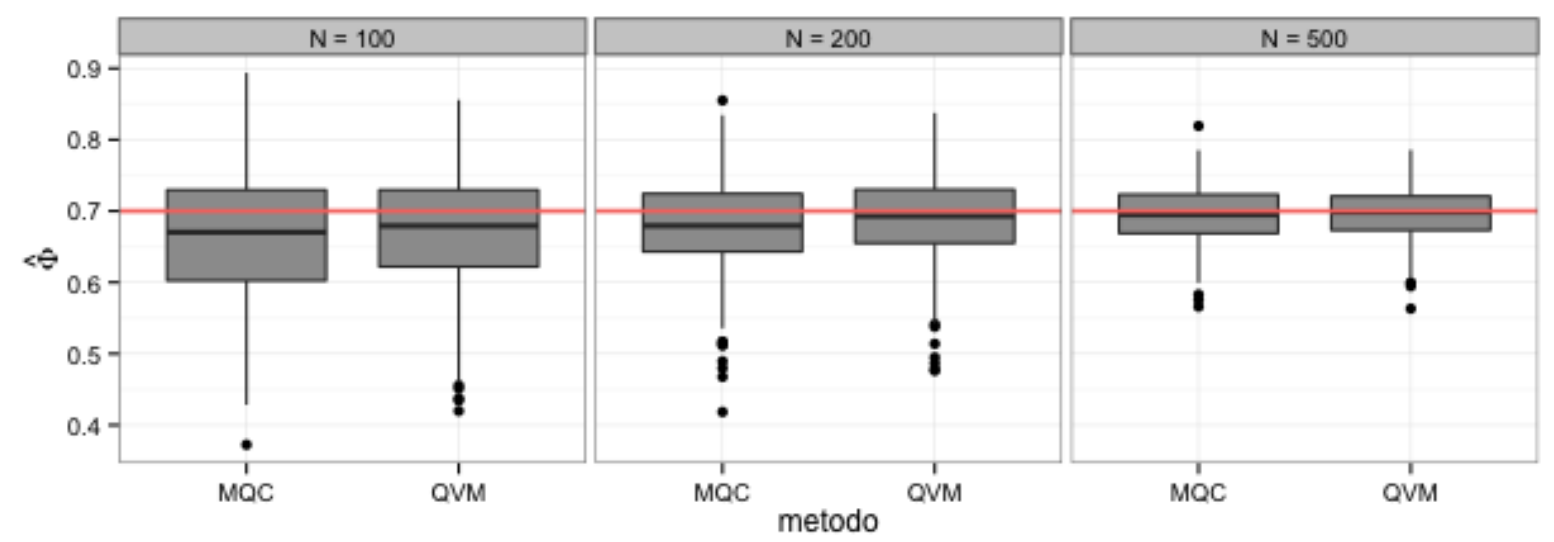

Figura 3.73: Boxplot das estimativas de $E\left[\phi_{t}\right]$ para o modelo gerado por $X_{t}=\phi_{t} \circ X_{t-1}+I_{t}$.

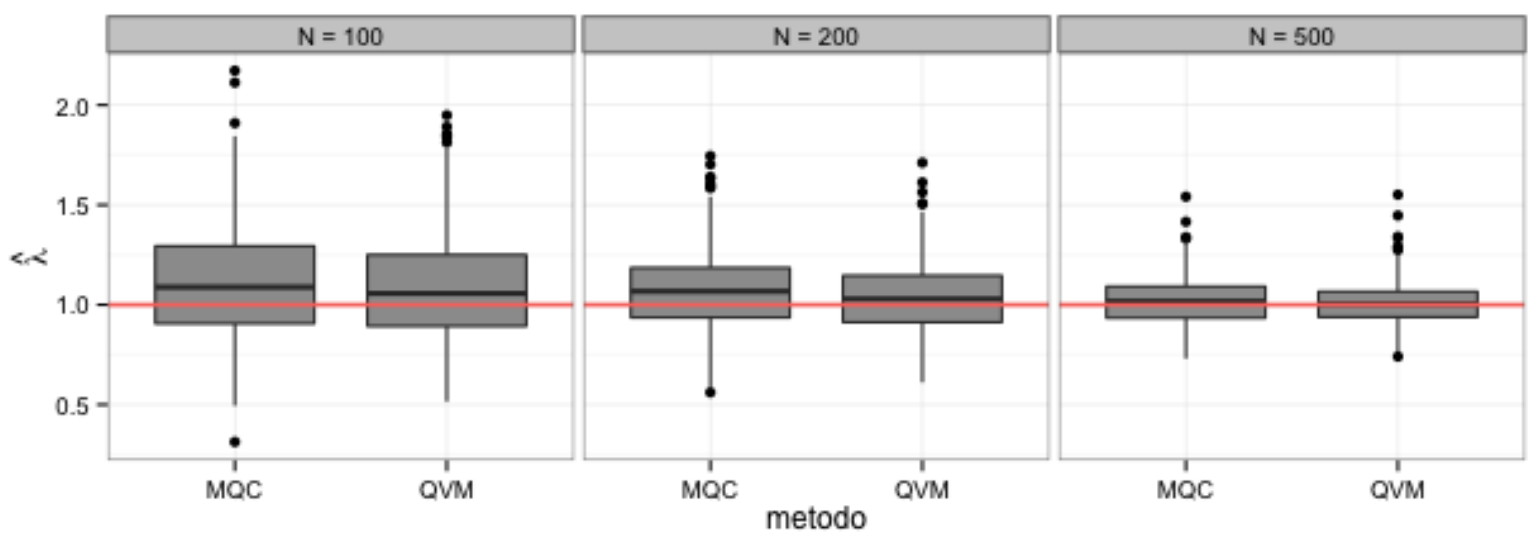

Figura 3.74: Boxplot das estimativas de $\lambda$ para o modelo gerado por $X_{t}=\phi_{t} \circ X_{t-1}+I_{t}$. 


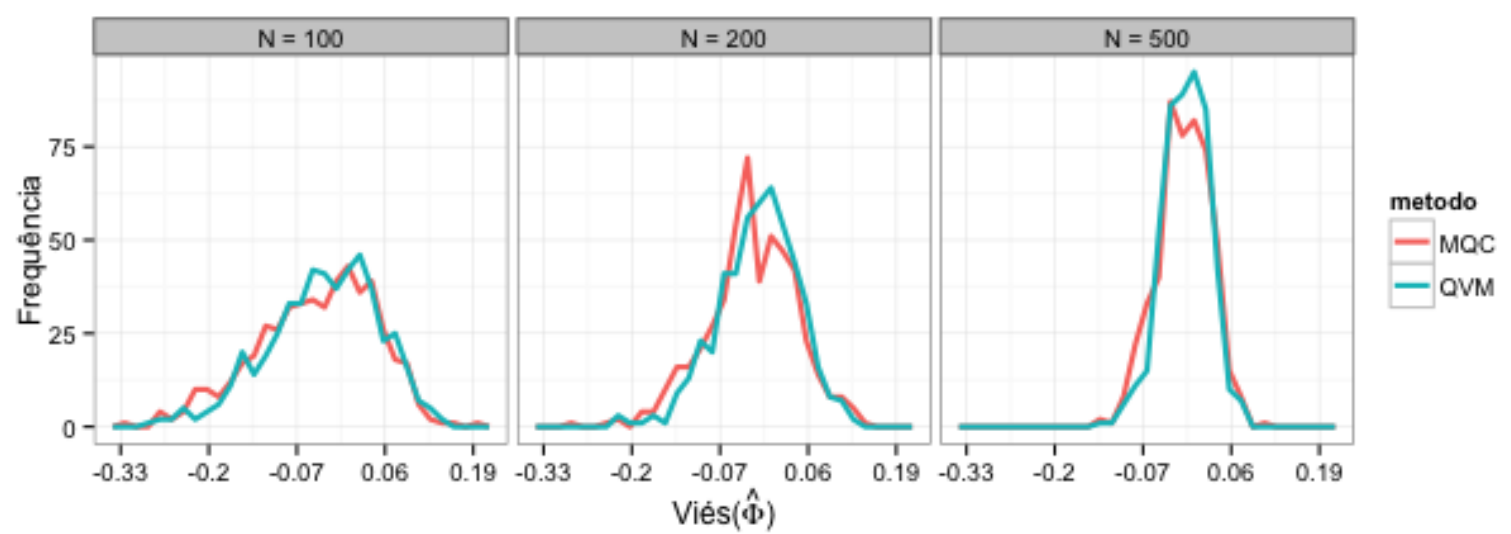

Figura 3.75: Histograma alisado do viés de $\hat{\Phi}$ para o modelo gerado por $X_{t}=\phi_{t} \circ X_{t-1}+I_{t}$.

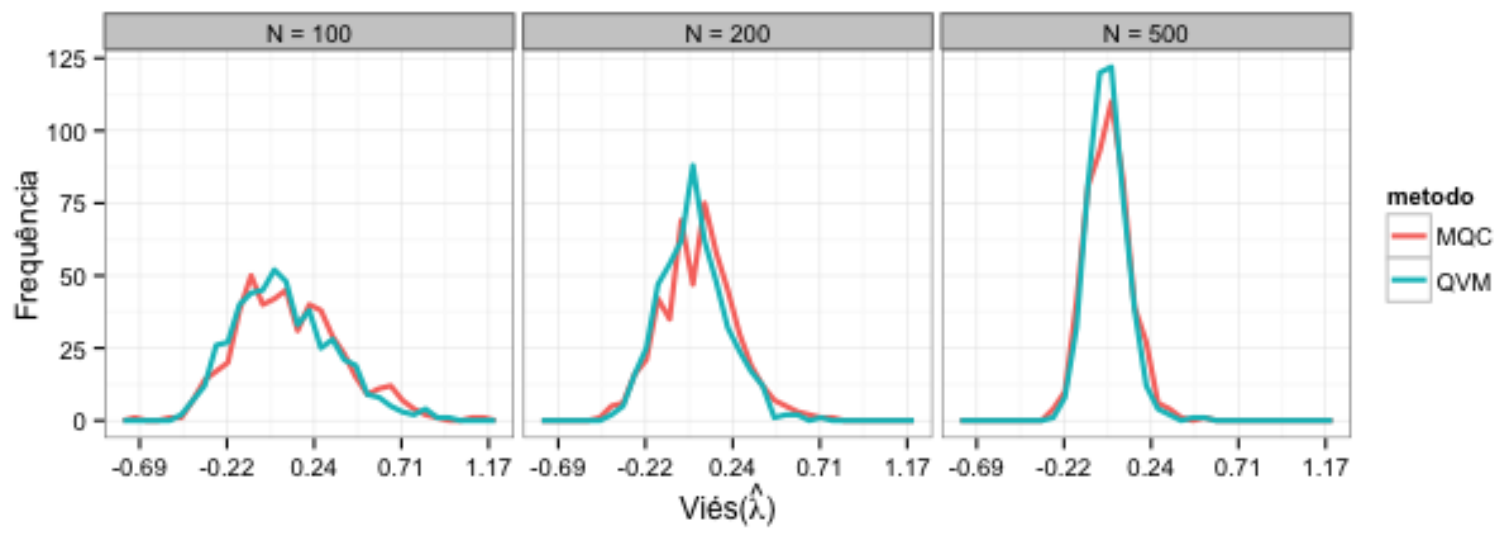

Figura 3.76: Histograma alisado do viés de $\hat{\lambda}$ para o modelo gerado por $X_{t}=\phi_{t} \circ X_{t-1}+I_{t}$.

As Figuras de 3.73 a 3.76 mostram que ambos métodos, MQC e QVM, produzem estimativas muito próximas do verdadeiro valor do parâmetro. À medida que o tamanho da série aumenta temos os valores das estimativas mais concentradas e a redução do viés. 


\begin{tabular}{ccccccc}
\hline \hline & & \multicolumn{2}{c}{ Viés $\left(\hat{\Phi}_{1}\right)$} & & \multicolumn{2}{c}{ Viés $(\hat{\lambda})$} \\
\cline { 3 - 4 } \cline { 6 - 7 } $\mathrm{N}$ & $\Phi_{1}$ & MQC & QVM & & MQC & QVM \\
\hline \multirow{2}{1000}{} & 0,3 & $-0,03017$ & $-0,02662$ & & 0,03514 & 0,03067 \\
& 0,5 & $-0,04000$ & $-0,02897$ & & 0,06394 & 0,04406 \\
& 0,7 & $-0,03810$ & $-0,02774$ & & 0,11430 & 0,08618 \\
200 & 0,3 & $-0,01227$ & $-0,00897$ & & 0,01026 & 0,00605 \\
& 0,5 & $-0,01762$ & $-0,01226$ & & 0,03042 & 0,02118 \\
& 0,7 & $-0,02007$ & $-0,01072$ & & 0,06247 & 0,03518 \\
500 & 0,3 & $-0,00547$ & $-0,00459$ & & 0,00658 & 0,00535 \\
& 0,5 & $-0,00818$ & $-0,00617$ & & 0,01695 & 0,01313 \\
& 0,7 & $-0,00630$ & $-0,00389$ & & 0,01585 & 0,00825 \\
\hline \hline
\end{tabular}

Tabela 3.9: Viés das estimações para o modelo RCINAR(1) $(\lambda=1)$ baseado em 500 réplicas.

\begin{tabular}{ccccccc}
\hline \hline & & \multicolumn{2}{c}{$\operatorname{EQM}\left(\hat{\Phi}_{1}\right)$} & & \multicolumn{2}{c}{$\operatorname{EQM}(\hat{\lambda})$} \\
\cline { 3 - 4 } \cline { 6 - 7 } $\mathrm{N}$ & $\Phi_{1}$ & $\mathrm{MQC}$ & QVM & & $\mathrm{MQC}$ & $\mathrm{QVM}$ \\
\hline \multirow{2}{*}{100} & 0,3 & 0,01352 & 0,01256 & & 0,03427 & 0,03228 \\
& 0,5 & 0,01058 & 0,00933 & & 0,04212 & 0,03712 \\
& 0,7 & 0,00909 & 0,00728 & & 0,09166 & 0,07628 \\
200 & 0,3 & 0,00563 & 0,00540 & & 0,01351 & 0,01299 \\
& 0,5 & 0,00549 & 0,00489 & & 0,02189 & 0,01951 \\
& 0,7 & 0,00470 & 0,00350 & & 0,04318 & 0,03178 \\
500 & 0,3 & 0,00251 & 0,00226 & & 0,00633 & 0,00580 \\
& 0,5 & 0,00263 & 0,00208 & & 0,00947 & 0,00770 \\
& 0,7 & 0,00154 & 0,00117 & & 0,01385 & 0,01045 \\
\hline \hline
\end{tabular}

Tabela 3.10: Erro Quadrático Médio das estimações para o modelo RCINAR(1) $(\lambda=1)$ baseado em 500 réplicas.

A Tabela 3.9 resume os vieses das simulações por tamanho da série, valor real do parâmetro e método de estimação. É possível verificar que em todas as combinações $\left(\Phi_{1}\right.$ versus $\left.N\right)$ que o estimador de QVM possui menor viés médio, em alguns casos o viés dado por QVM é metade do viés dado por MQC.

A superioridade do estimador de QVM também pode ser notada na Tabela 3.10, onde temos os EQM medidos. Os valores observados de EQM do estimador dado por QVM são sempre menores que os EQM dados pelo estimador MQC.

A grandeza do verdadeiro valor do parâmetro não parece influenciar na qualidade do estimador; essa afirmação é verificada a medida que se avaliam as diferenças entre estimadores de QVM e MQC entre os distintos valores de $\Phi$. 
Processo RCINAR(2): $X_{t}=\phi_{1}^{(t)} \circ X_{t-1}+\phi_{2}^{(t)} \circ X_{t-2}, E\left[\phi_{1}^{(t)}\right]=0,3$ e $E\left[\phi_{2}^{(t)}\right]=0,3$.

Iniciamos aqui as análises dos processos RCINAR de ordem dois. A partir de agora a avaliação será sobre dois parâmetros de ordens distintas, $\phi_{1}^{(t)}$ e $\phi_{2}^{(t)}$ geradas independentemente.

A Figura 3.77 apresenta o histograma e autocorrelação amostral de $\phi_{1}^{(t)}$; em seguida temos a Figura 3.78 com histograma e autocorrelação de $\phi_{2}^{(t)}$. Uma trajetória da série $X_{t}$ gerada é apresentada na Figura 3.79.

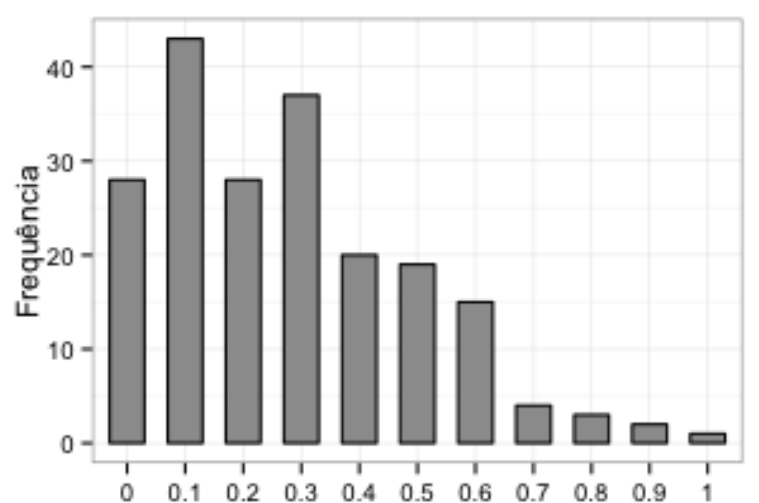

(a)

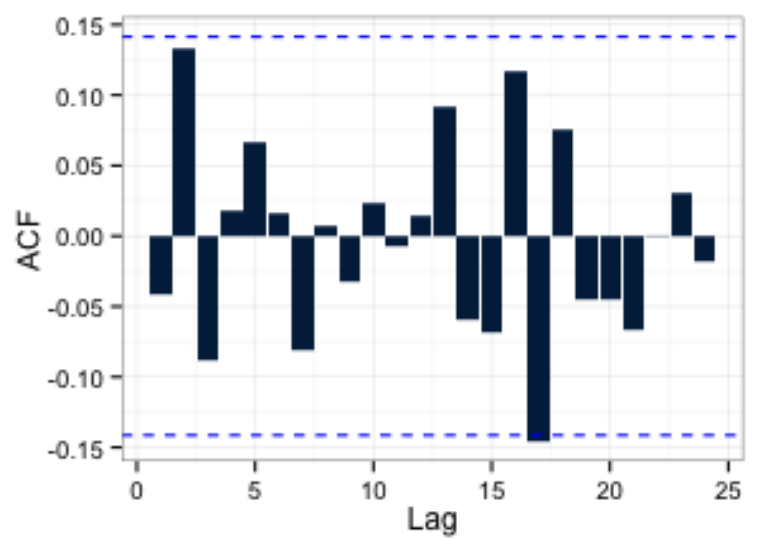

Figura 3.77: Histograma e ACF do $\phi_{1}^{(t)}$ simulado, RCINAR(2).

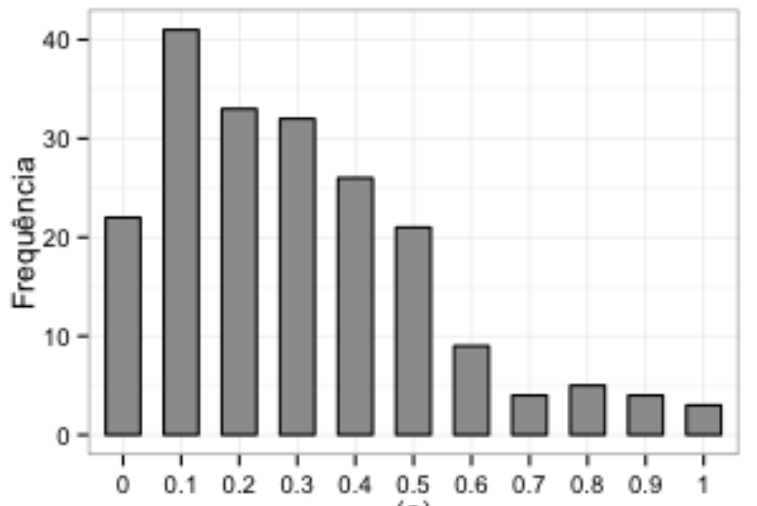

(a)

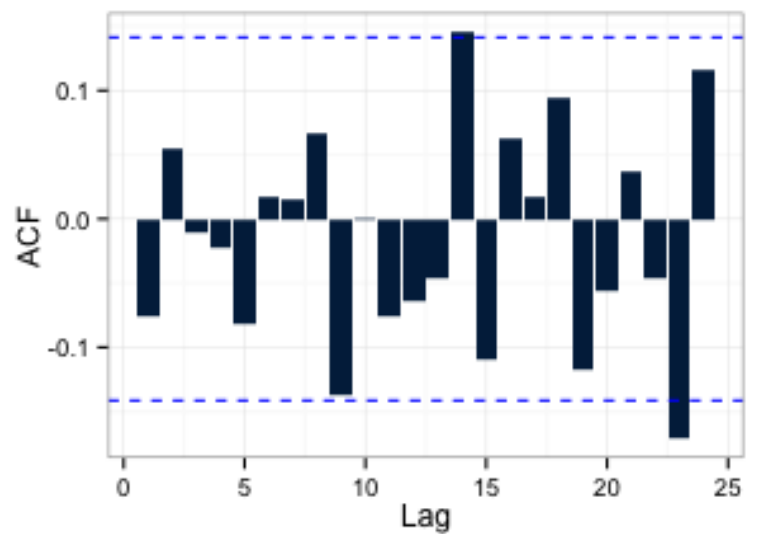

Figura 3.78: Histograma e $A C F$ do $\phi_{2}^{(t)}$ simulado, RCINAR(2).

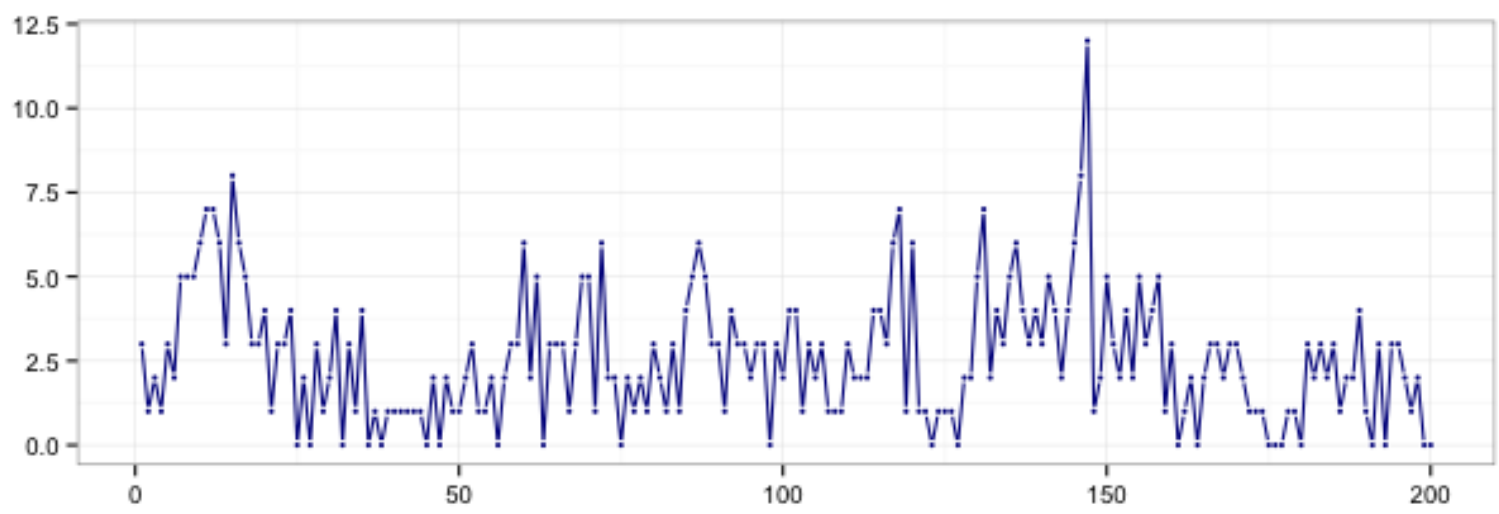

Figura 3.79: Gráfico de uma série simulada, $R C I N A R(\mathscr{L}): E\left[\phi_{1}^{(t)}\right]=0,3, E\left[\phi_{2}^{(t)}\right]=0,3$ e $\lambda=1$. 


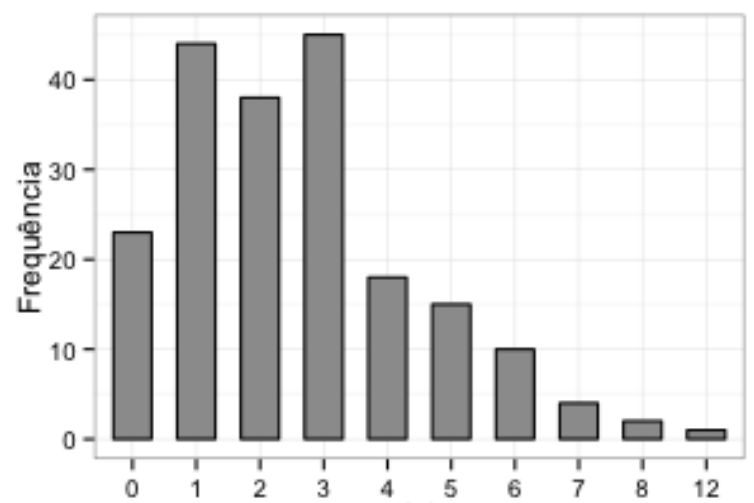

(a)

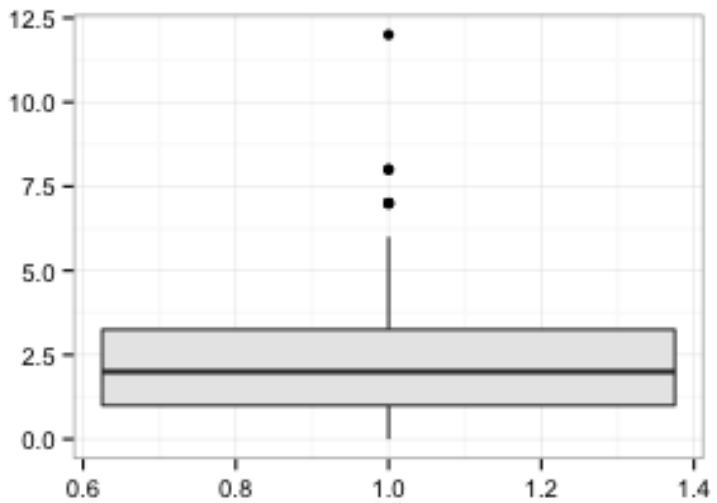

(b)

Figura 3.80: Histograma e boxplot da série simulada, $R C I N A R(2): E\left[\phi_{1}^{(t)}\right]=0,3, E\left[\phi_{2}^{(t)}\right]=0,3$ e $\lambda=1$.
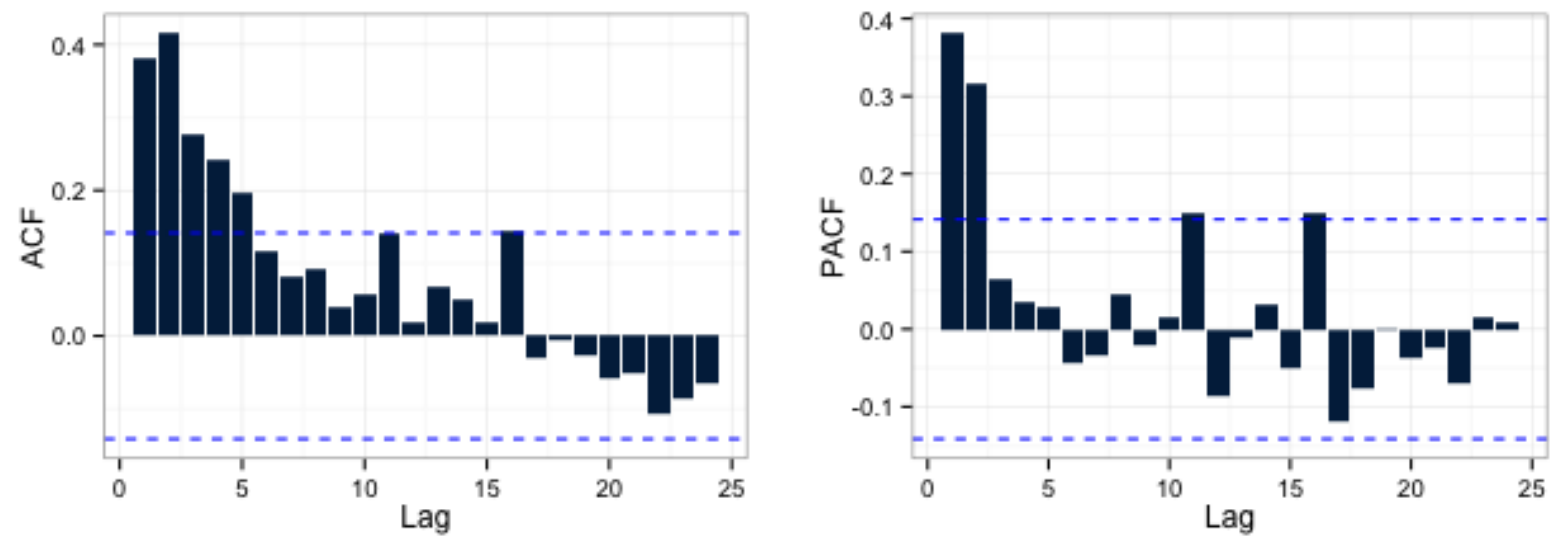

Figura 3.81: Autocorrelação e Autocorrelação parcial amostrais $\operatorname{RCINAR(2):~} E\left[\phi_{1}^{(t)}\right]=0,3, E\left[\phi_{2}^{(t)}\right]=0,3$ e $\lambda=1$.

O histograma da série e o boxplot são apresentados na Figura 3.80; a autocorrelação e autocorrelação parcial são mostradas na Figura 3.81. A ACF e PACF mostram um comportamento adequado para o modelo gerado, RCINAR de ordem dois. Em seguida, as Figuras 3.82, 3.83 e 3.84 mostram os gráficos boxplot das estimativas, dadas por MQC e QVM, de $\hat{\Phi}_{1}, \hat{\Phi}_{2}$ e $\hat{\lambda}$, respectivamente. É possível ver que ambos métodos de estimação produzem estimativas bastante próximas do verdadeiro valor do parâmetro, no entanto, as estimativas dadas por QVM parecem mais concentradas. 


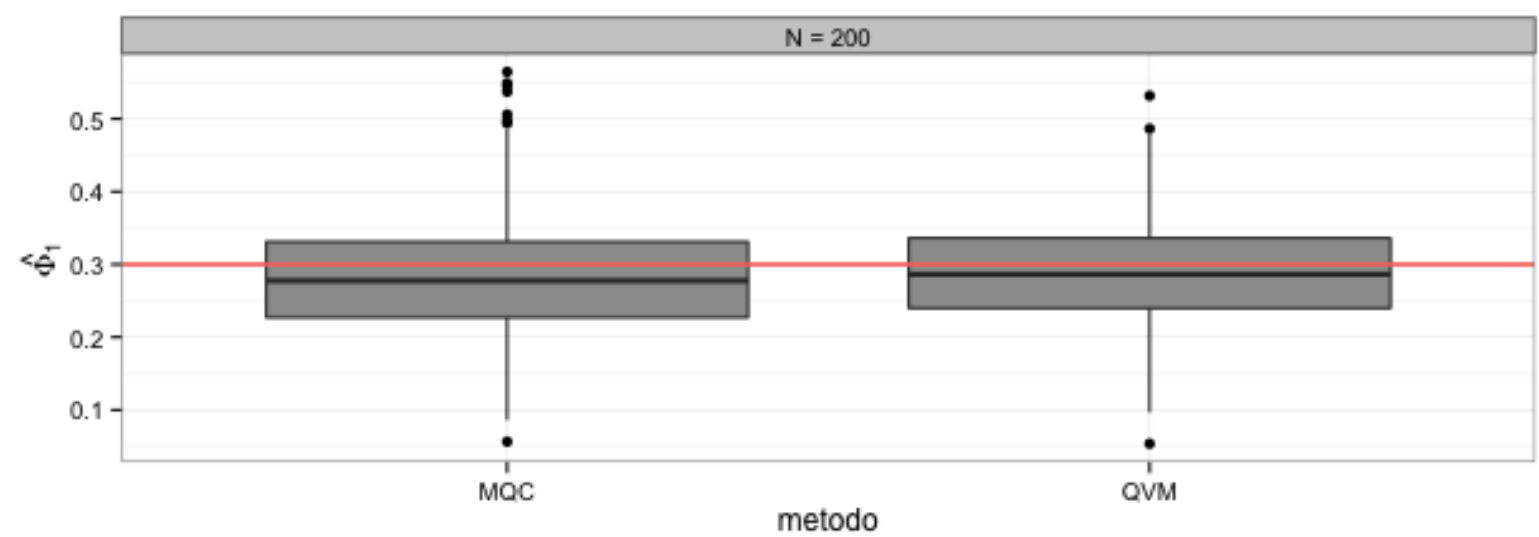

Figura 3.82: Boxplot das estimativas de $E\left[\phi_{1}^{(t)}\right]$ para o modelo gerado.

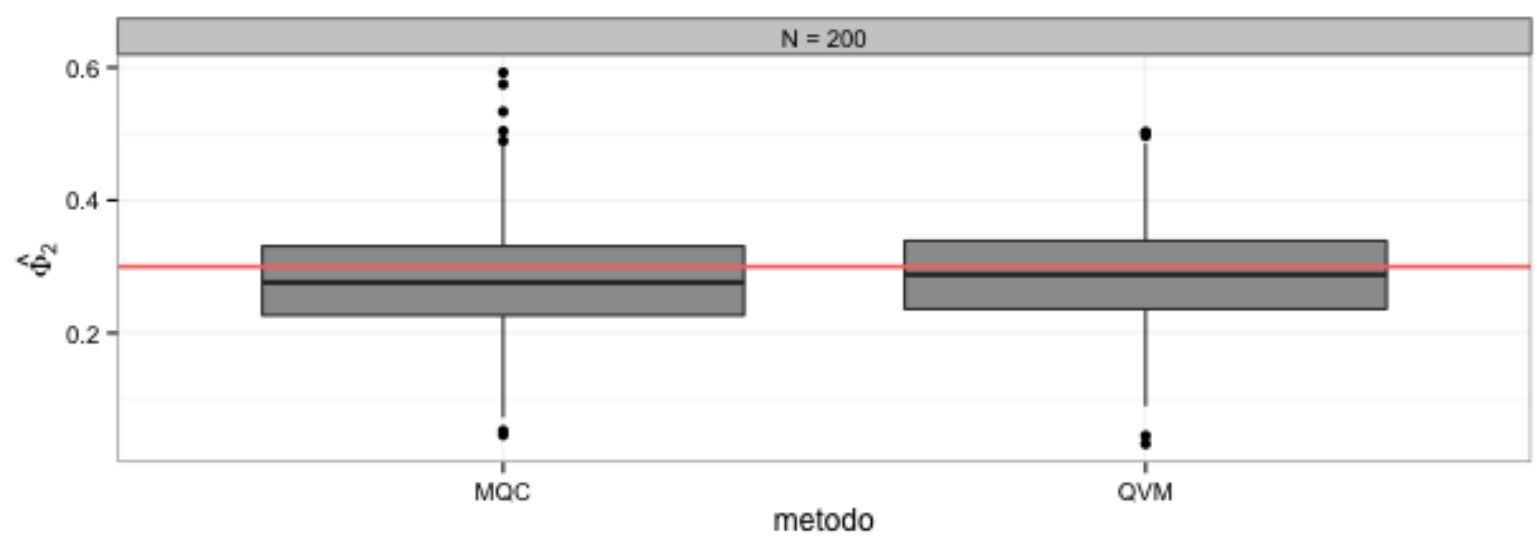

Figura 3.83: Boxplot das estimativas de $E\left[\phi_{2}^{(t)}\right]$ para o modelo gerado.

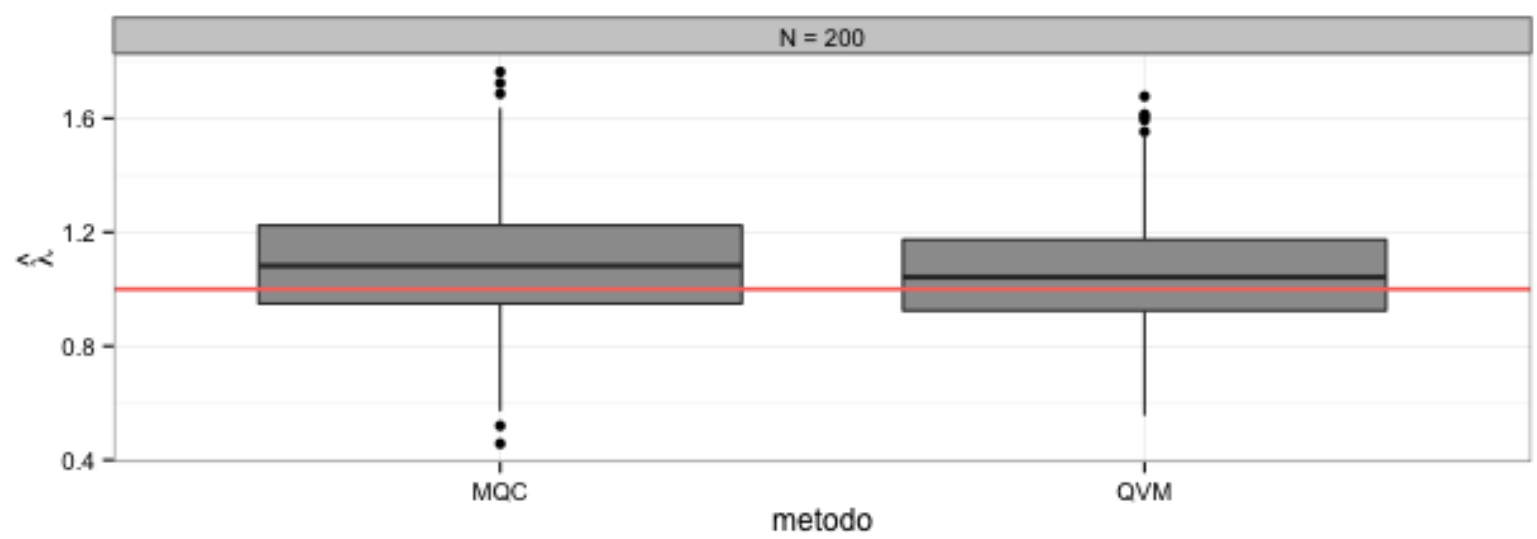

Figura 3.84: Boxplot das estimativas de $\lambda$ para o modelo gerado. 
O histograma alisado do viés de $\hat{\Phi}_{1}$ é apresentado na Figura 3.85, enquanto nas Figuras 3.85 e 3.87 temos o histograma alisado dos vieses de $\hat{\Phi}_{2}$ e $\hat{\lambda}$, respectivamente. Os tres histogramas mostram que ambos estimadores utilizados, MQC e QVM, tem vieses muito próximos.

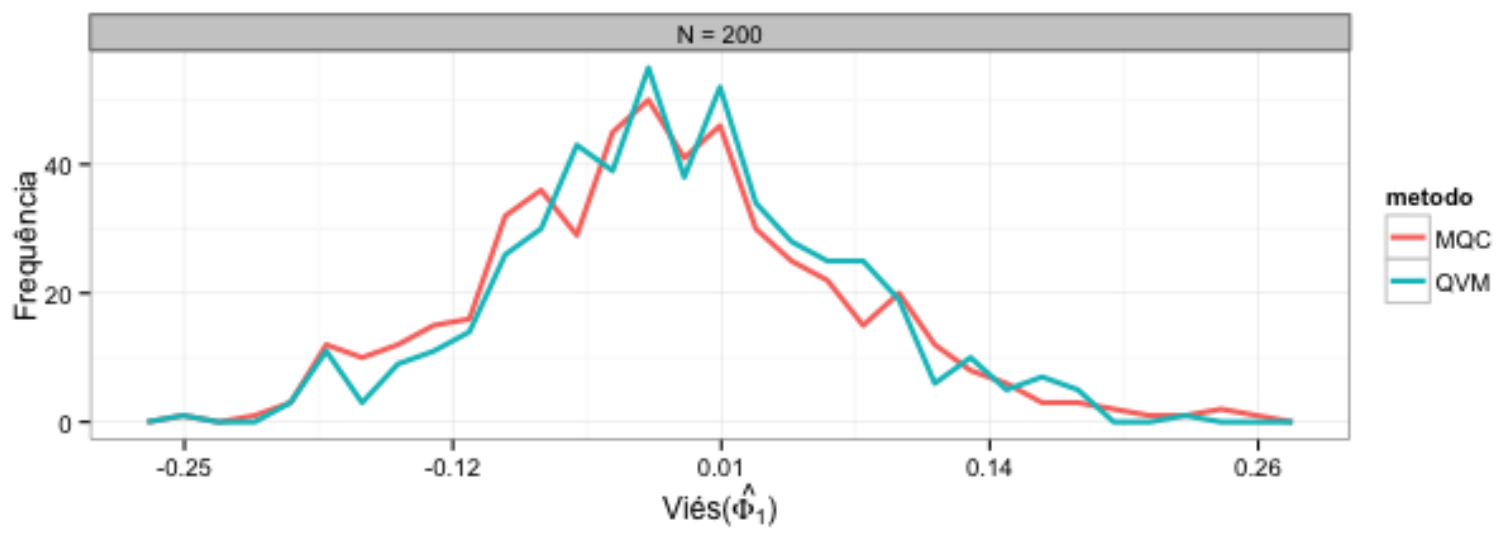

Figura 3.85: Histograma alisado do viés de $\hat{\Phi}_{1}$ para o modelo gerado.

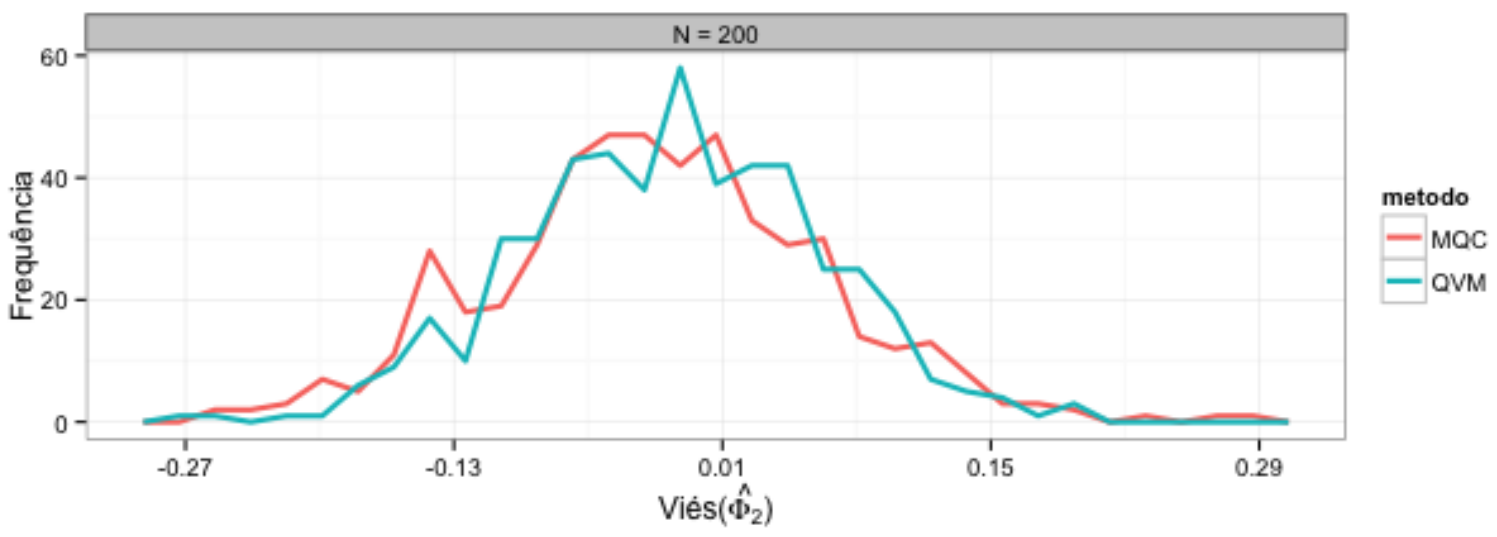

Figura 3.86: Histograma alisado do viés de $\hat{\Phi}_{2}$ para o modelo gerado.

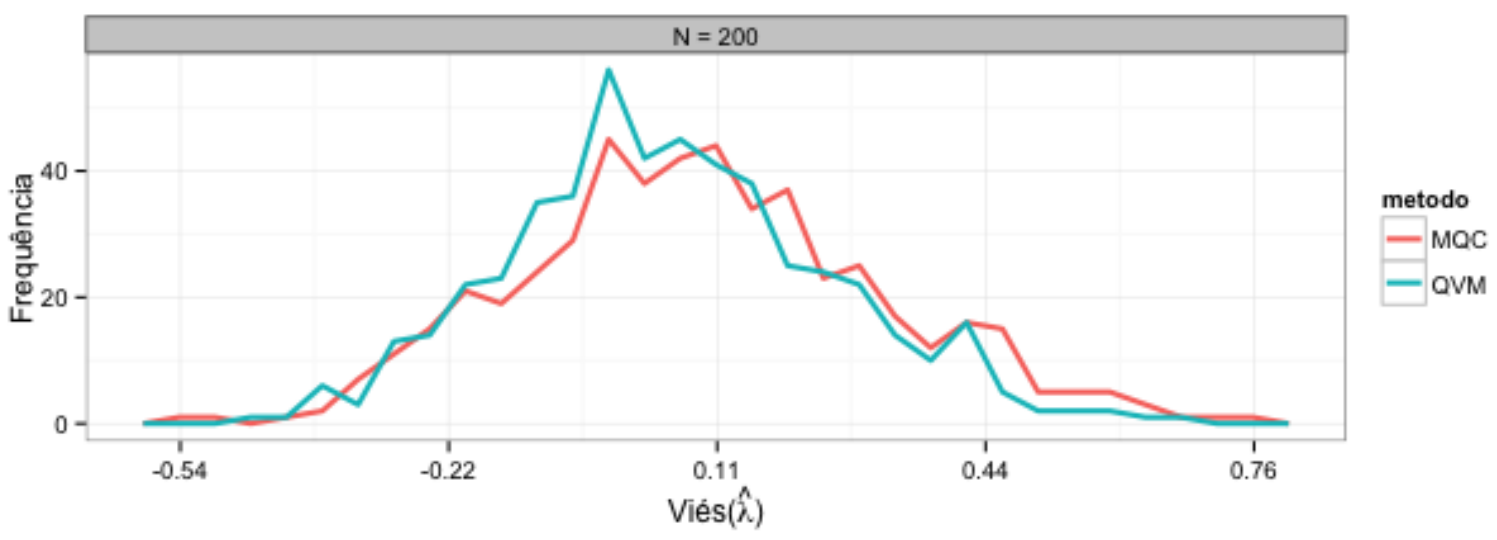

Figura 3.87: Histograma alisado do viés de $\hat{\lambda}$ para o modelo gerado. 
Processo RCINAR(2): $X_{t}=\phi_{1}^{(t)} \circ X_{t-1}+\phi_{2}^{(t)} \circ X_{t-2}, E\left[\phi_{1}^{(t)}\right]=0,3$ e $E\left[\phi_{2}^{(t)}\right]=0,5$.

O histograma e ACF do parâmetro $\phi_{1}^{(t)}$ são apresentados na Figura 3.88; notamos que o comportamento do histograma se assemelha a distribuição adotada para $\phi_{1}^{(t)}$, mostrada na Figura 3.52. O mesmo é possível ver para o parâmetro $\phi_{2}^{(t)}$ na Figura 3.88, que apresenta novamente o histograma e ACF.

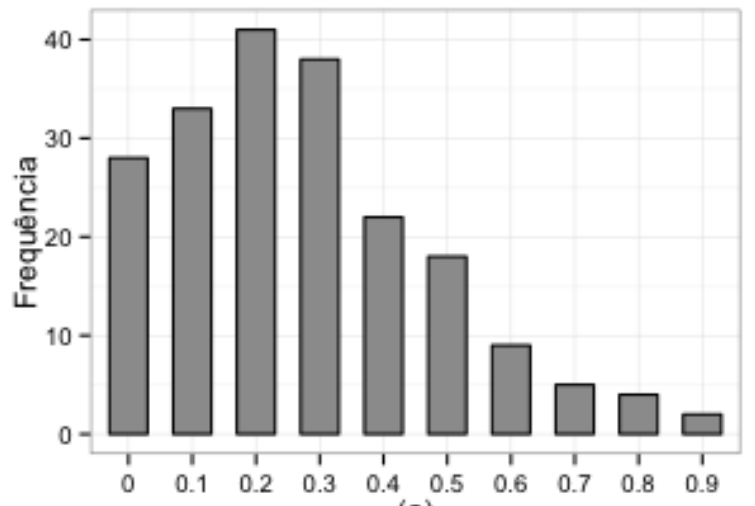

(a)

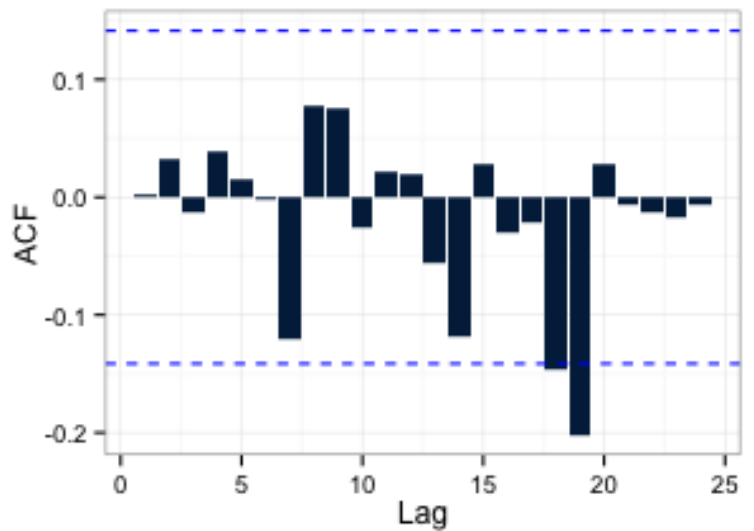

Figura 3.88: Histograma e $A C F$ do $\phi_{1}^{(t)}$ simulado, RCINAR(2).

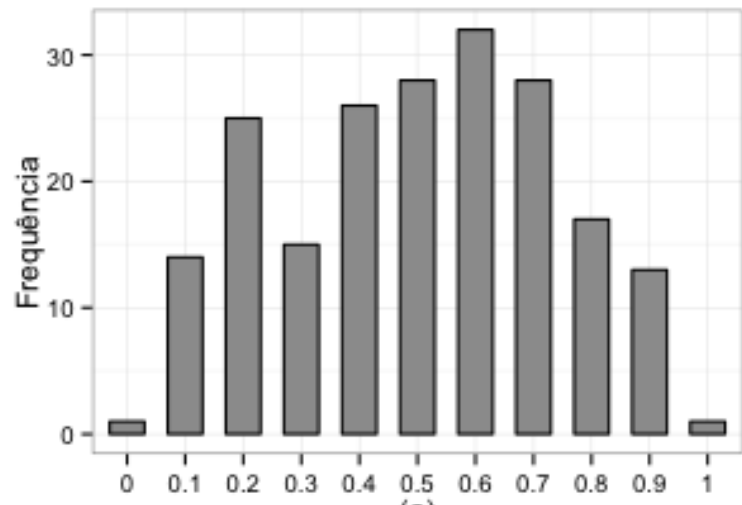

(a)

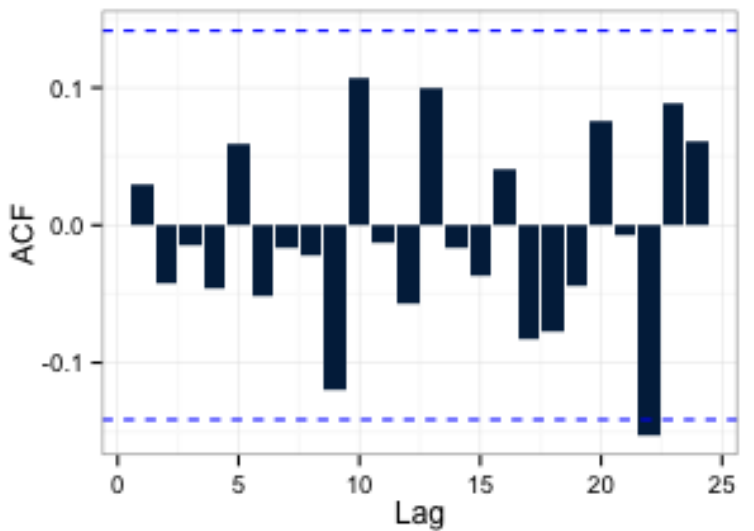

Figura 3.89: Histograma e $A C F$ do $\phi_{2}^{(t)}$ simulado, RCINAR(2).

A seguir, é apresentado na Figura 3.90, o comportamento temporal de uma trajetória simulada para o processo RCINAR de ordem dois. Vemos também o histograma da trajetória seguido do boxplot na Figura 3.91; é possível verificar que a trajetória gerada possui média próxima à esperada, dada por $(1-0.3-0.5)^{-1}=5$.

A ACF e PACF da trajetória gerada são apresentadas na Figura 3.92, onde notamos o comportamento esperado para um processo de ordem dois: decaimento na ACF e ordens um e dois significantes na PACF. 


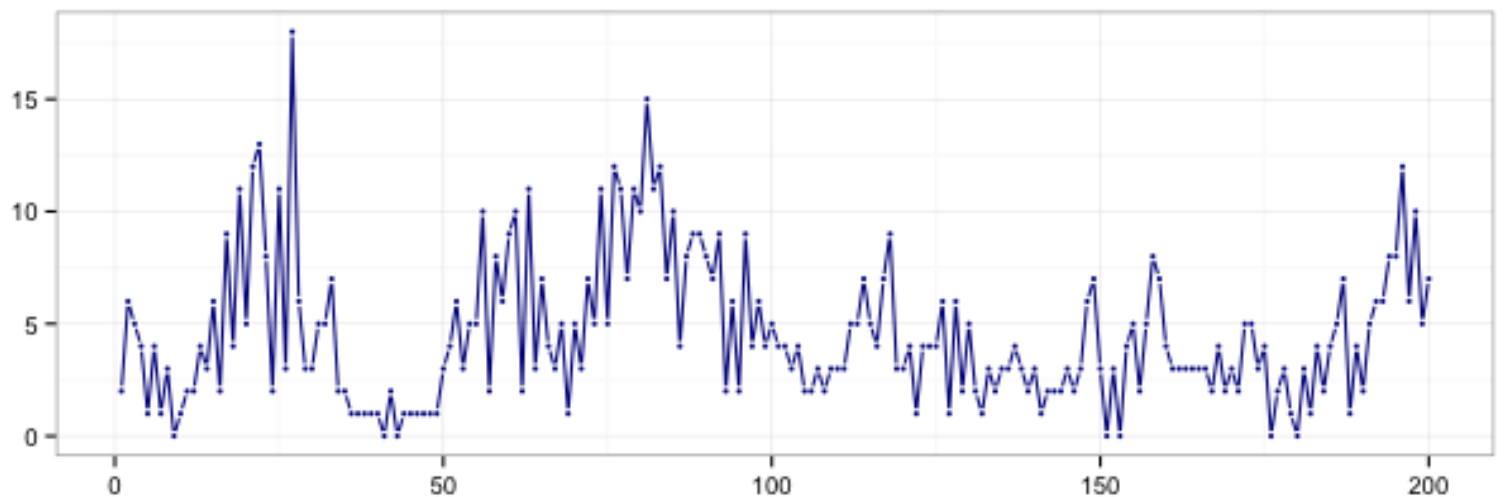

Figura 3.90: Gráfico de uma série simulada, $R C I N A R(2): E\left[\phi_{1}^{(t)}\right]=0,3, E\left[\phi_{2}^{(t)}\right]=0,5$ e $\lambda=1$.

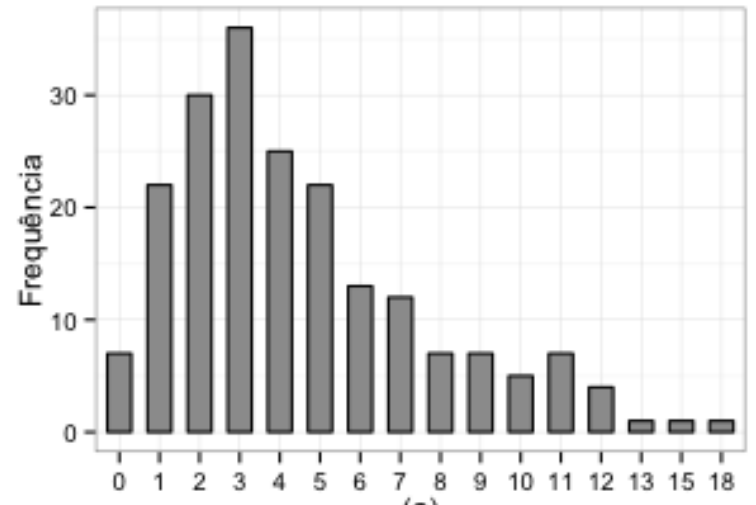

(a)

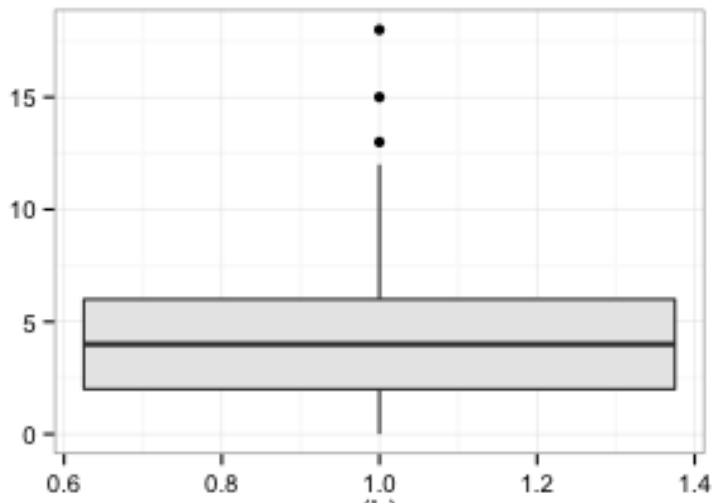

(b)

Figura 3.91: Histograma e boxplot da série simulada, $R C I N A R(2): E\left[\phi_{1}^{(t)}\right]=0,3, E\left[\phi_{2}^{(t)}\right]=0,5$ e $\lambda=1$.
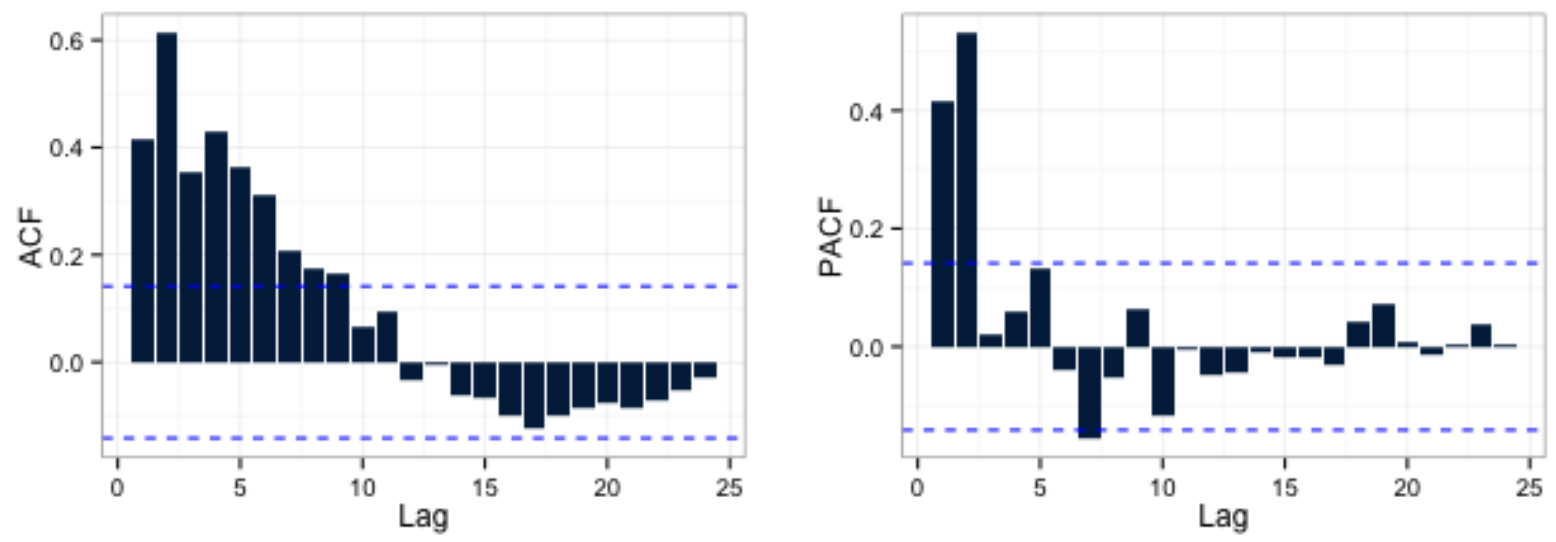

Figura 3.92: Autocorrelação e Autocorrelação parcial amostrais $\operatorname{RCINAR(2):~} E\left[\phi_{1}^{(t)}\right]=0,3, E\left[\phi_{2}^{(t)}\right]=0,5$ $e \lambda=1$. 
Os estimadores de MQC e QVM produzem boas estimativas para o parâmetro $\Phi_{1}$, é o que evidencia a Figura 3.93; notamos uma pequena vantagem do estimador QVM, que possui estimativas mais concentradas. A avaliação das estimativas do parâmetro $\Phi_{2}$ é realizada através do boxplot da Figura 3.94, em que notamos que a mediana de estimativas dadas por QVM são mais próximas de 0.5. O mesmo é notado na Figura 3.95, em que o estimador QVM apresenta melhor performance.

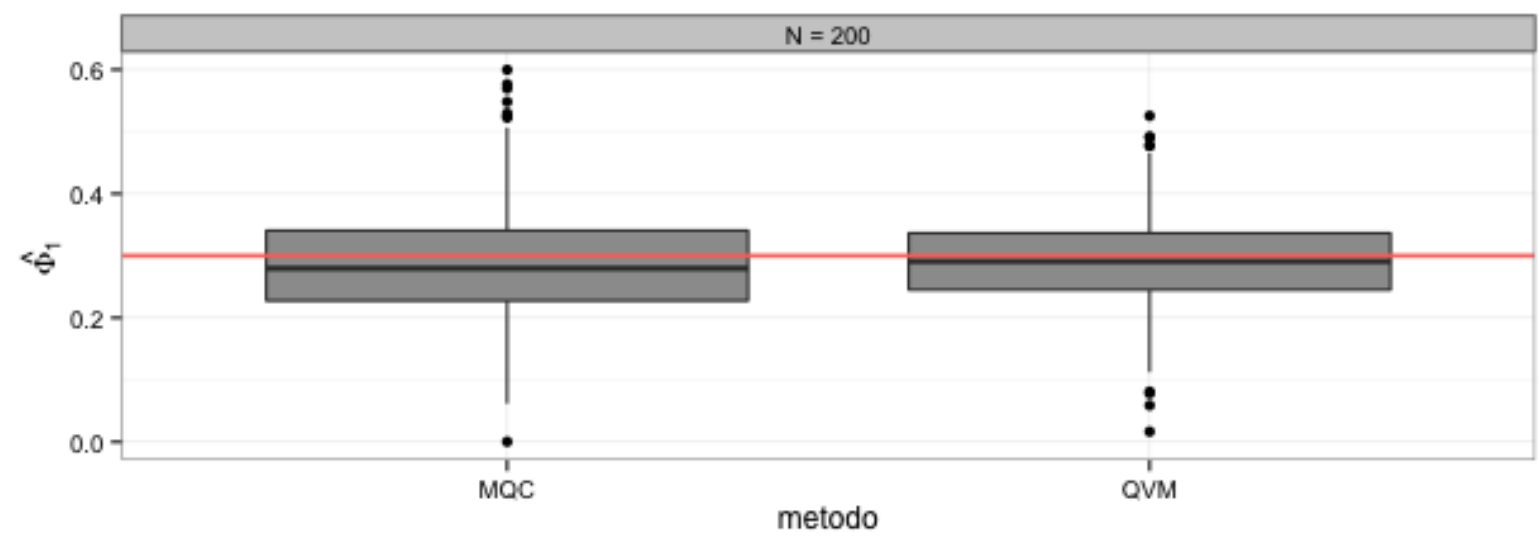

Figura 3.93: Boxplot das estimativas de $E\left[\phi_{1}^{(t)}\right]$ para o modelo gerado.

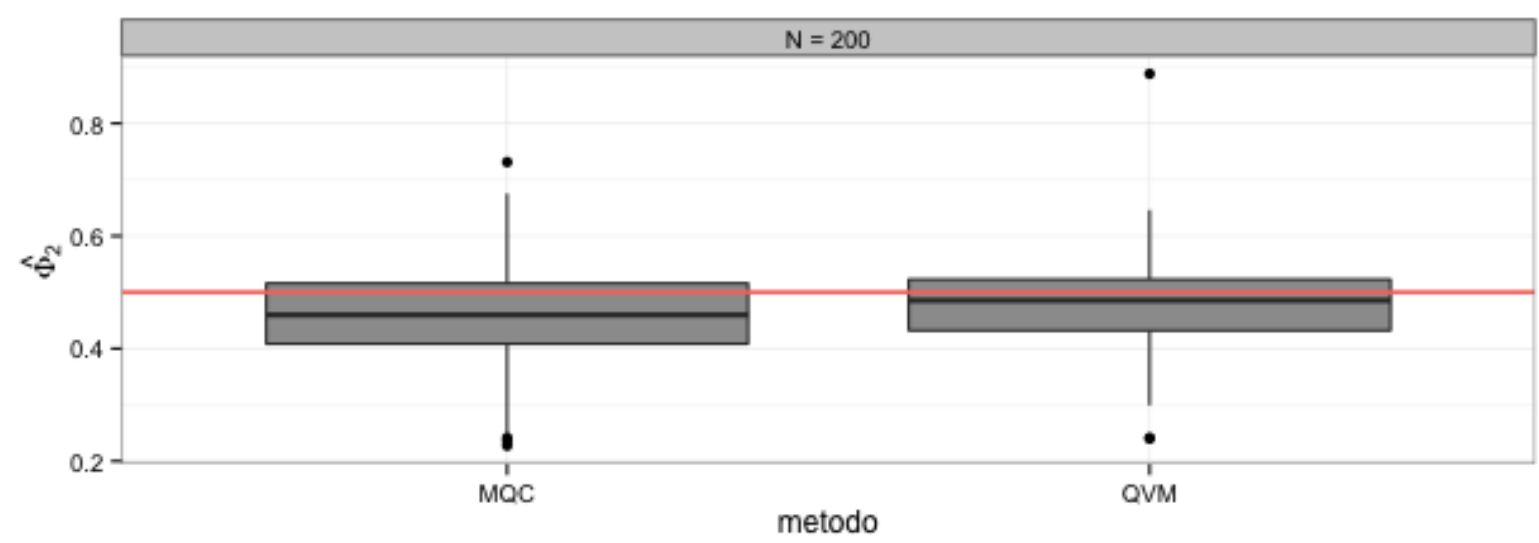

Figura 3.94: Boxplot das estimativas de $E\left[\phi_{2}^{(t)}\right]$ para o modelo gerado.

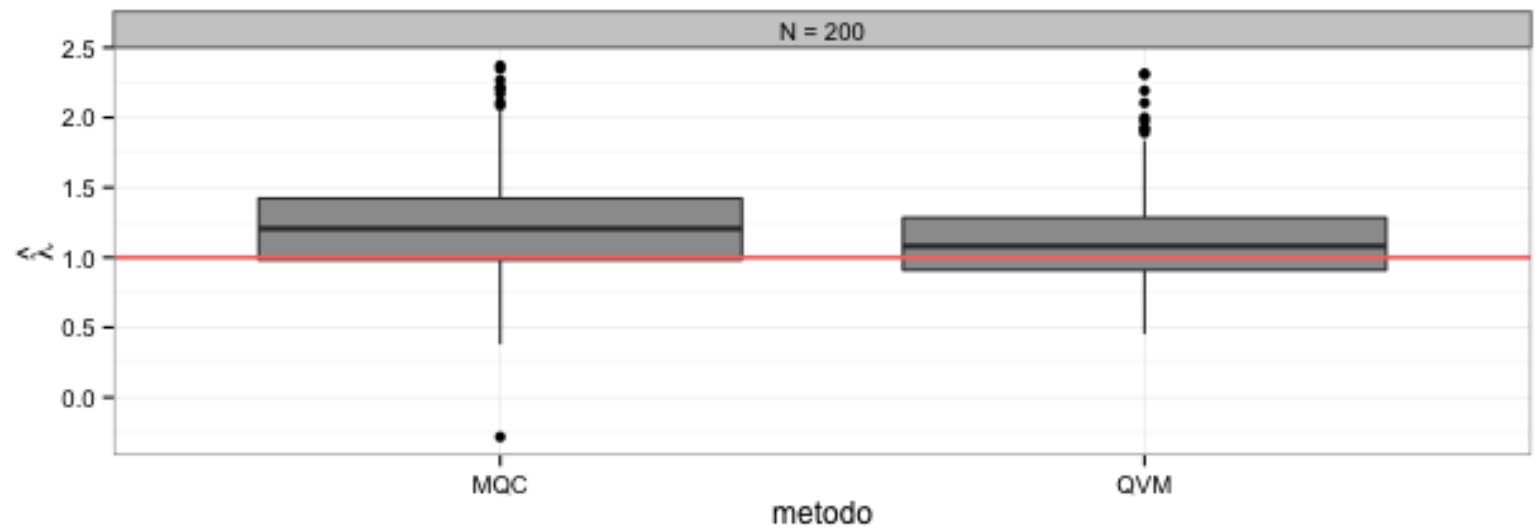

Figura 3.95: Boxplot das estimativas de $\lambda$ para o modelo gerado. 


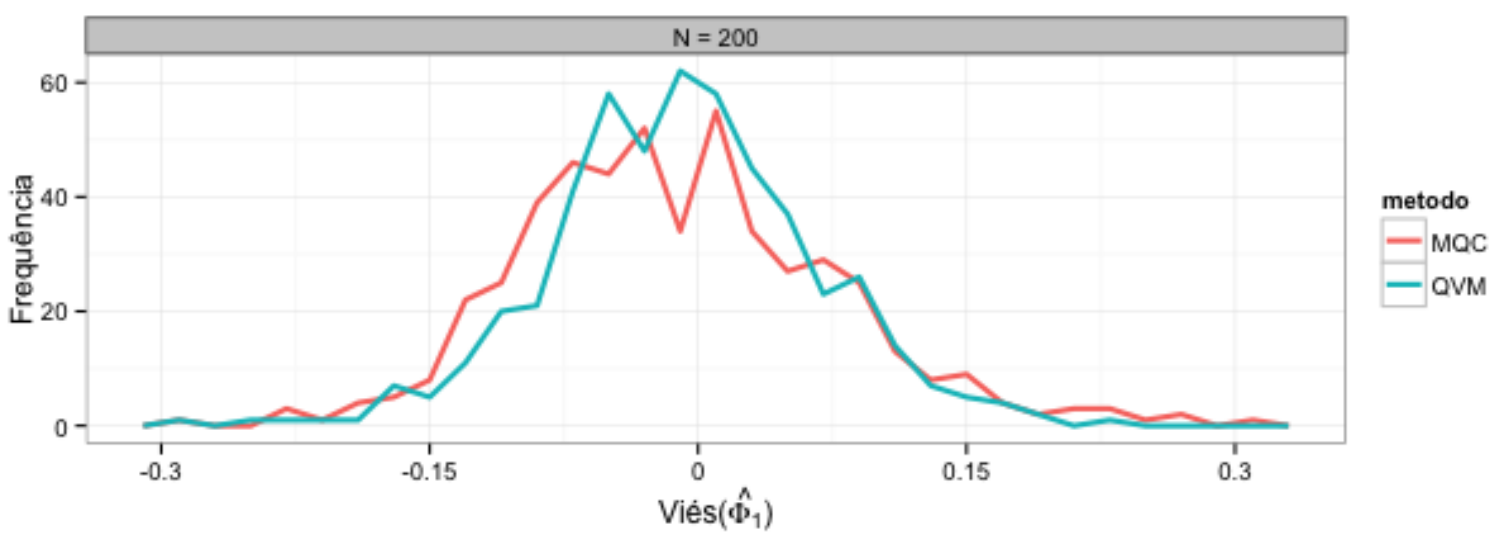

Figura 3.96: Histograma alisado do viés de $\hat{\Phi_{1}}$ para o modelo gerado.

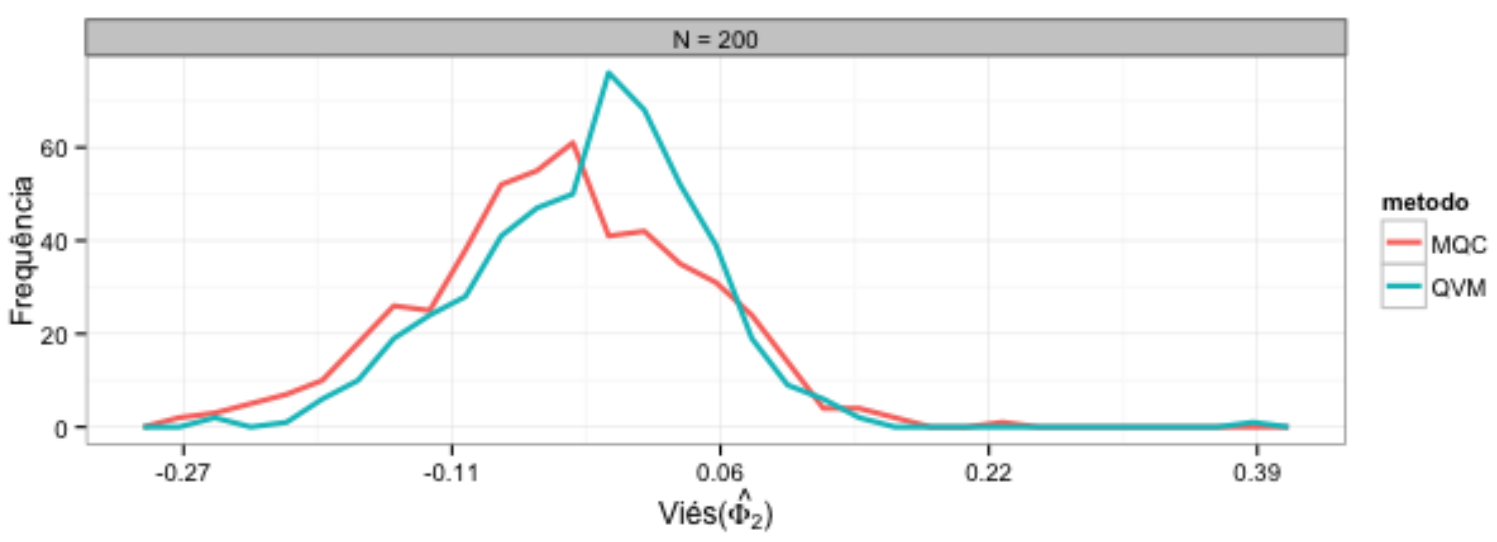

Figura 3.97: Histograma alisado do viés de $\hat{\Phi}_{2}$ para o modelo gerado.

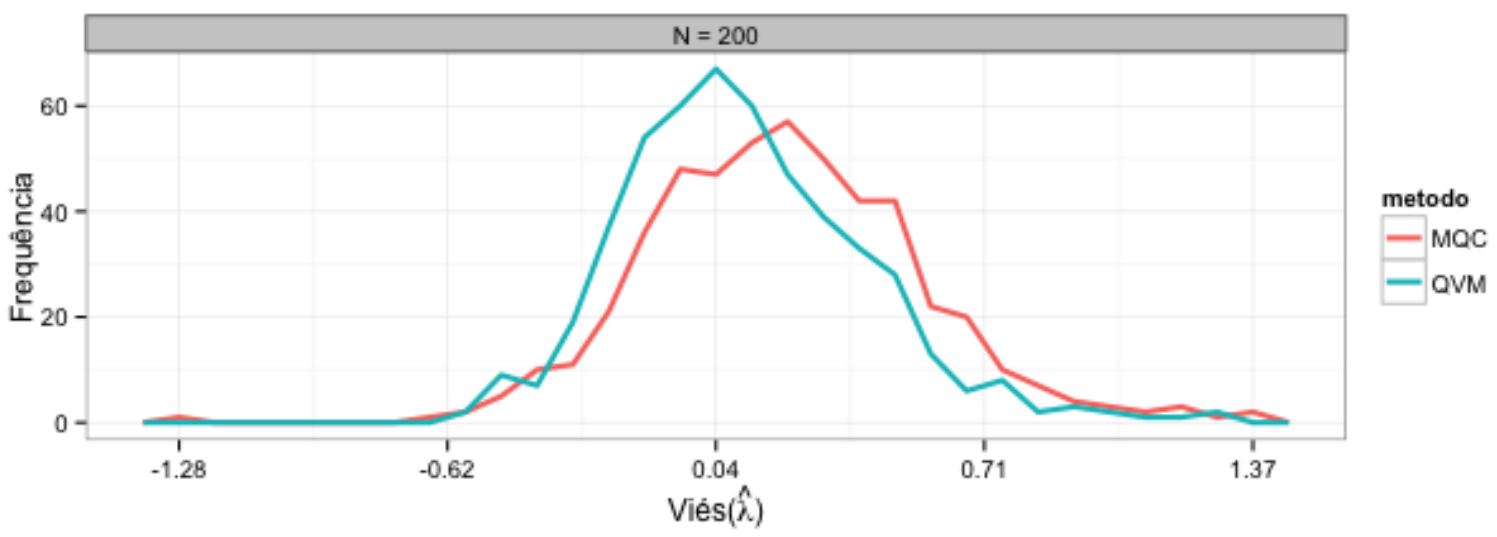

Figura 3.98: Histograma alisado do viés de $\hat{\lambda}$ para o modelo gerado.

A avaliação desse processo é encerrado com o comportamento dos vieses. Vemos nas Figuras 3.96, 3.97 e 3.98, o histograma alisado dos vieses de $\hat{\Phi}_{1}, \hat{\Phi}_{2}$ e $\hat{\lambda}$, respectivamente. Aqui é mais clara a diferença entre a performance dos estimadores de QVM e MQC, destacando principalmente os vieses de $\hat{\Phi}_{2}$ e $\hat{\lambda}$, onde os estimadores de QVM produzem estimativas mais assertivas. 
Processo RCINAR(2): $X_{t}=\phi_{1}^{(t)} \circ X_{t-1}+\phi_{2}^{(t)} \circ X_{t-2}, E\left[\phi_{1}^{(t)}\right]=0,5$ e $E\left[\phi_{2}^{(t)}\right]=0,3$.

Vemos o comportamento de uma distribuição Beta evidenciada no histograma da Figura 3.99, e na Figura 3.100, como esperado; as mesmas figuras também apresentam a ACF de $\phi_{1}^{(t)}$ e $\phi_{2}^{(t)}$ gerados. A seguir, apresentamos uma trajetória simulada do processo RCINAR na Figura 3.101.

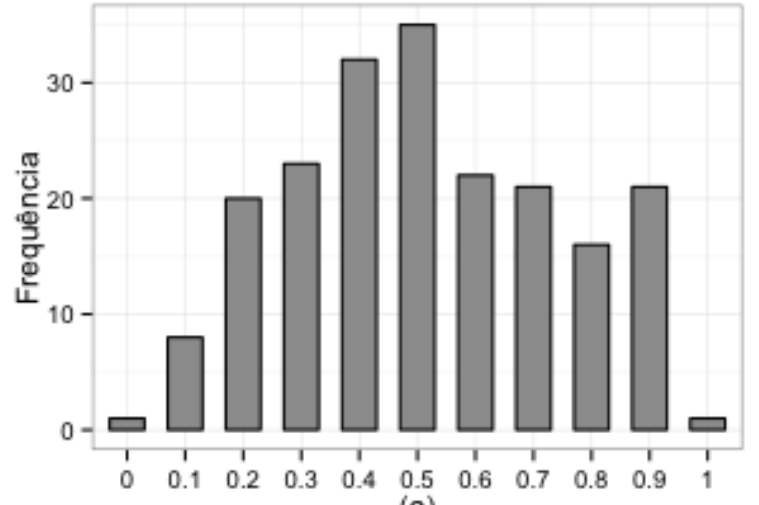

(a)

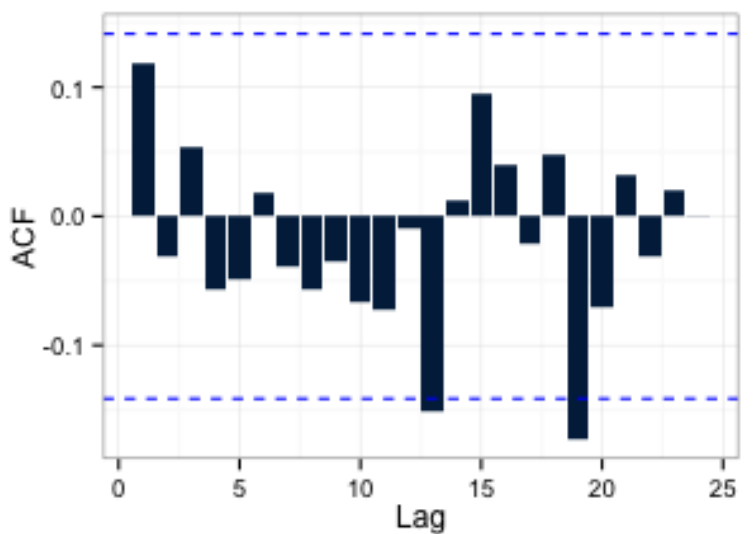

Figura 3.99: Histograma e ACF do $\phi_{1}^{(t)}$ simulado, RCINAR(2).

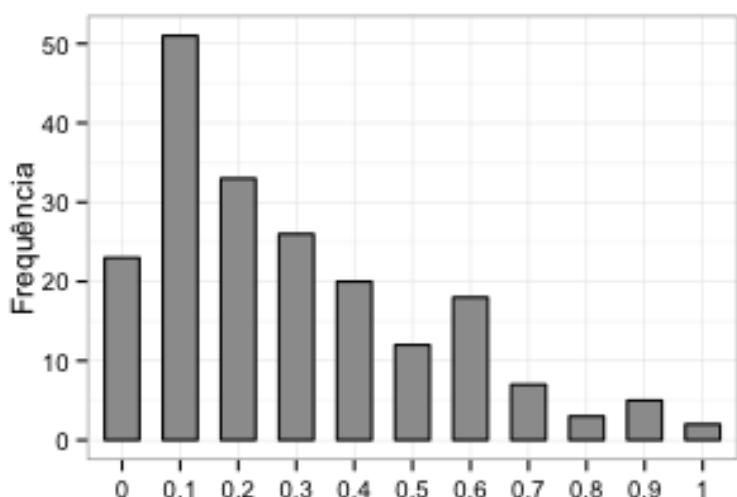

(a)

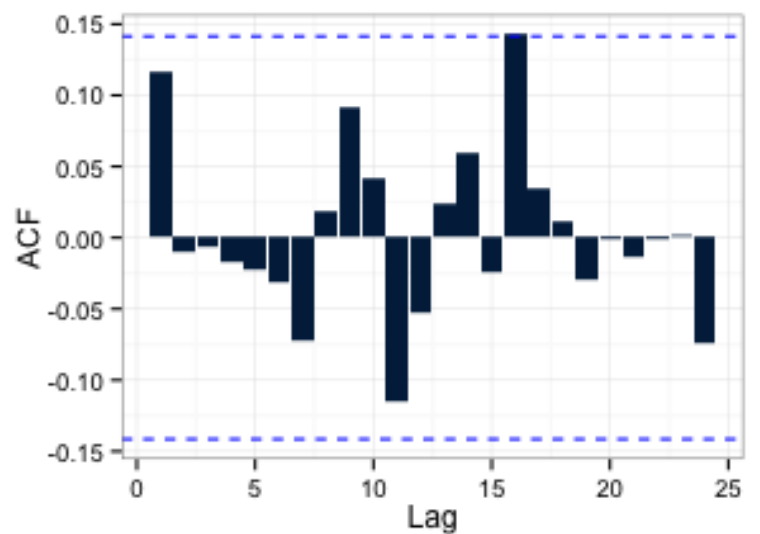

Figura 3.100: Histograma e $A C F$ do $\phi_{2}^{(t)}$ simulado, $R C I N A R(2)$.

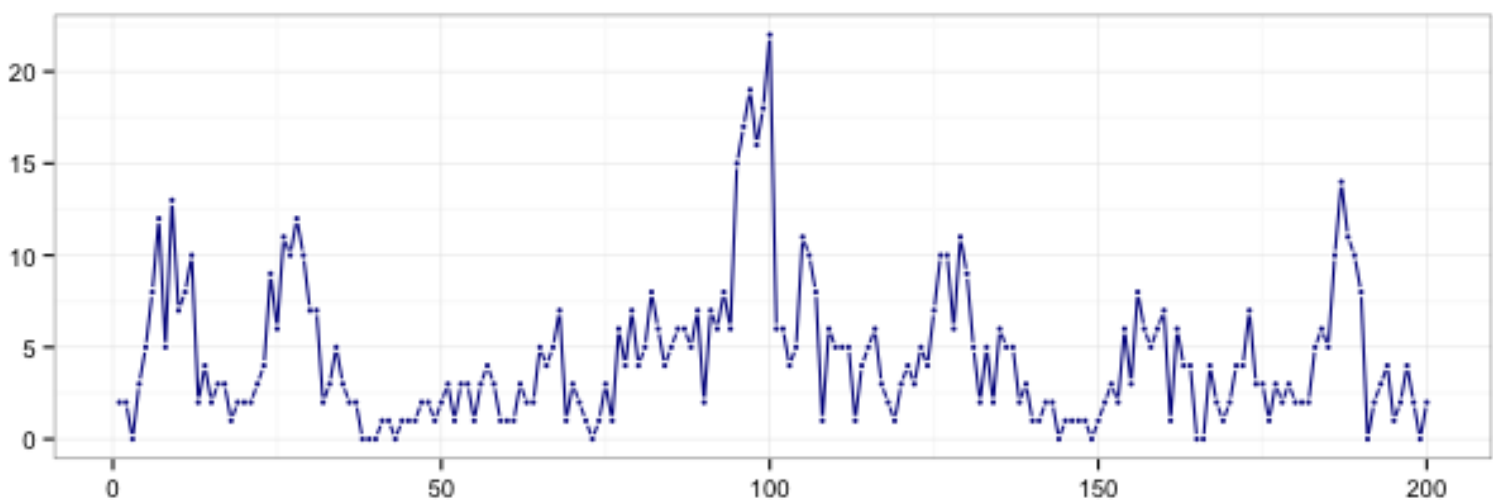

Figura 3.101: Gráfico de uma série simulada, $R C I N A R(2): E\left[\phi_{1}^{(t)}\right]=0,5, E\left[\phi_{2}^{(t)}\right]=0,3$ e $\lambda=1$. 


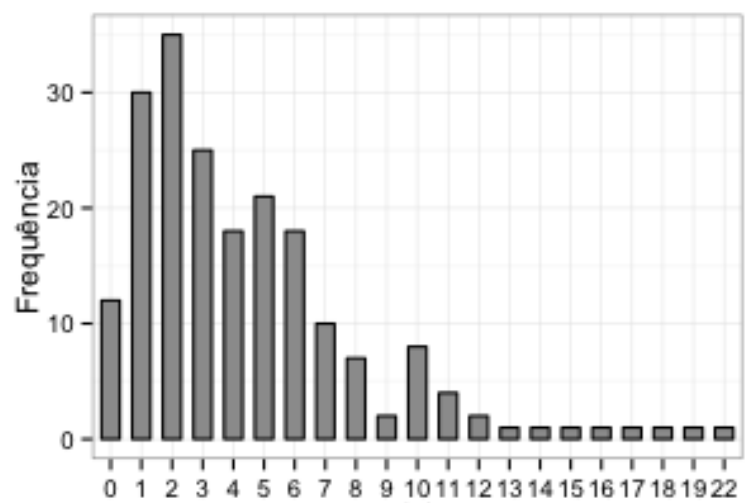

(a)

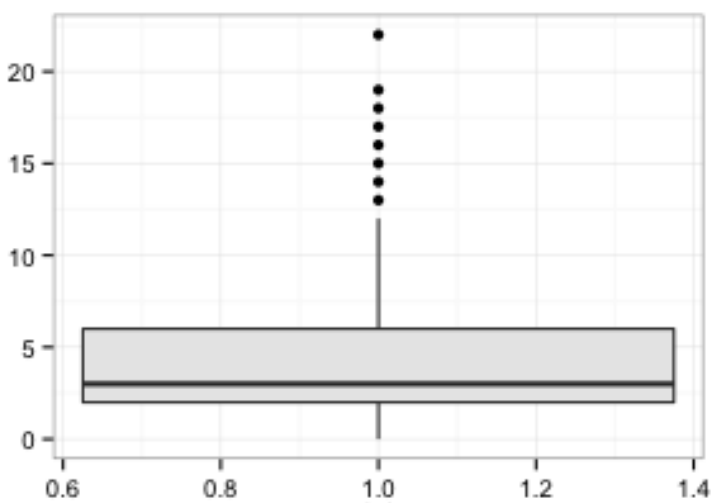

(b)

Figura 3.102: Histograma e boxplot da série simulada, $R C I N A R(2): E\left[\phi_{1}^{(t)}\right]=0,5, E\left[\phi_{2}^{(t)}\right]=0,3$ e $\lambda=1$.
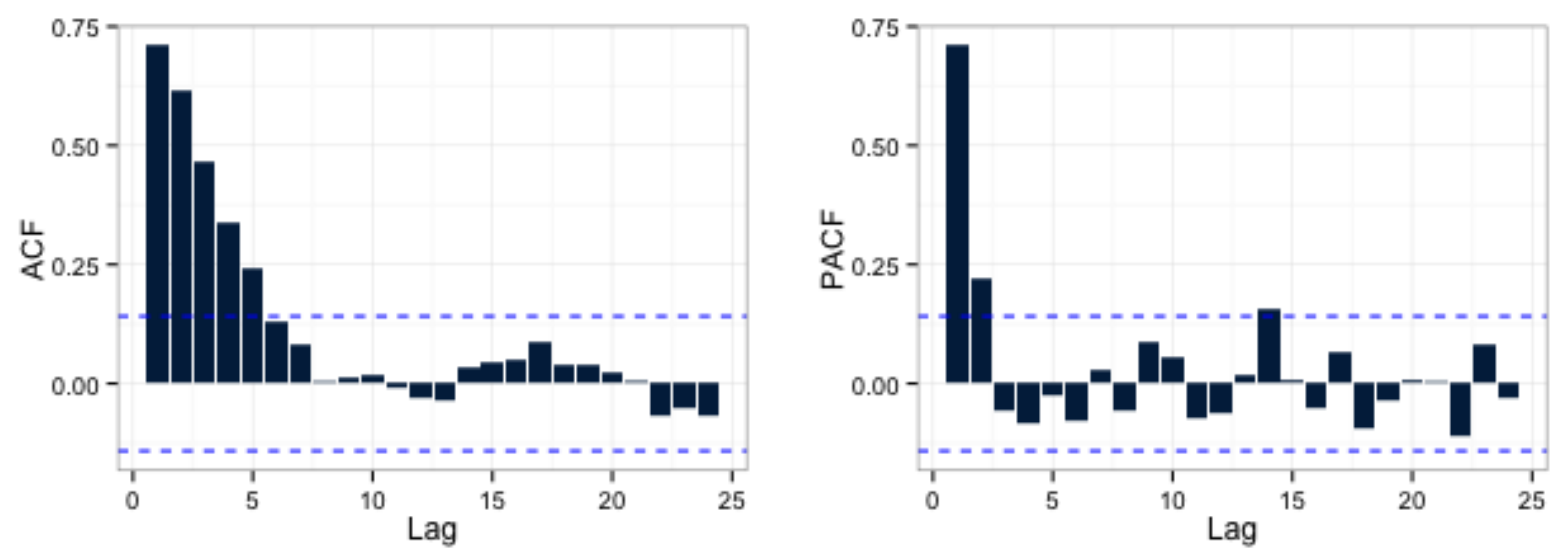

Figura 3.103: Autocorrelação e Autocorrelação Parcial Amostrais $R C I N A R(2): E\left[\phi_{1}^{(t)}\right]=0,5, E\left[\phi_{2}^{(t)}\right]=0,3$ e $\lambda=1$.

Acima, na Figura 3.102 temos o histograma (a) e o boxplot (b) de uma trajetória simulada. O histograma mostra assimetria à esquerda, natural de uma distribuição Poisson. A Figura 3.103 apresenta a ACF e PACF de uma trajetória simulada, mostrando o comportamento de um processo RCINAR de ordem dois.

Os estimadores de QVM novamente apresentam melhores resultados, comprovado na Figura 3.104, 3.105 e 3.106. Com exceção dos outliers observados, as estimativas dadas por QVM são mais concentradas próximo ao verdadeiro valor do parâmetro. 


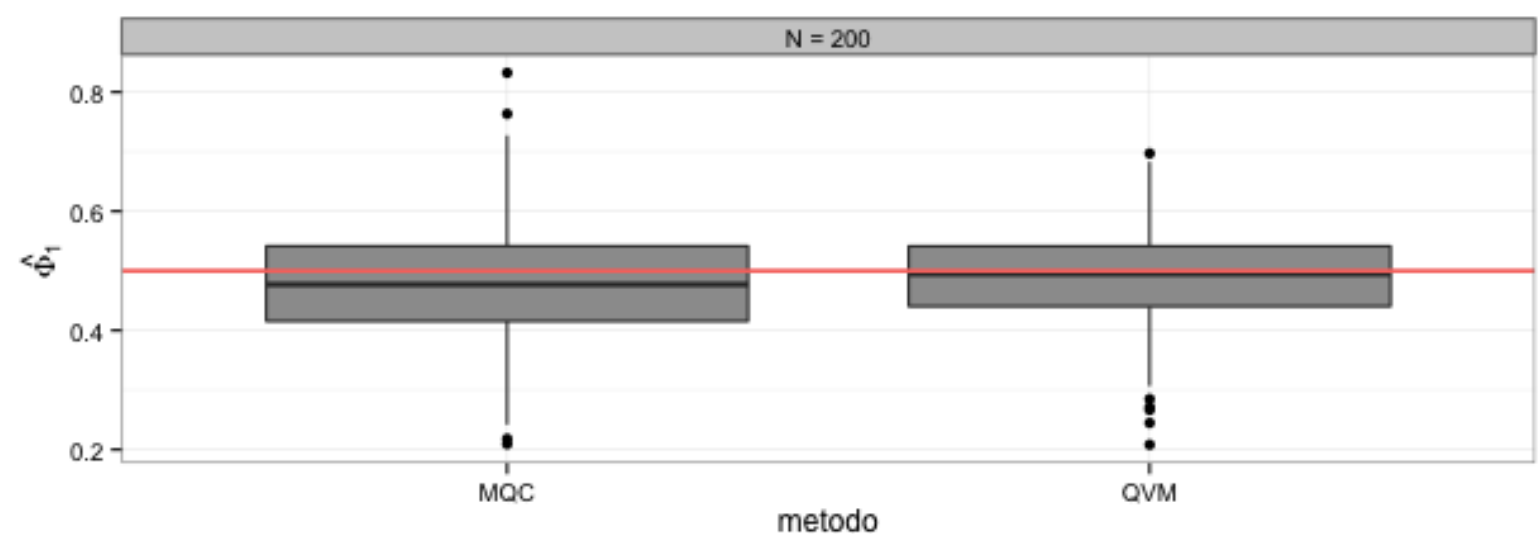

Figura 3.104: Boxplot das estimativas de $E\left[\phi_{1}^{(t)}\right]$ para o modelo gerado.

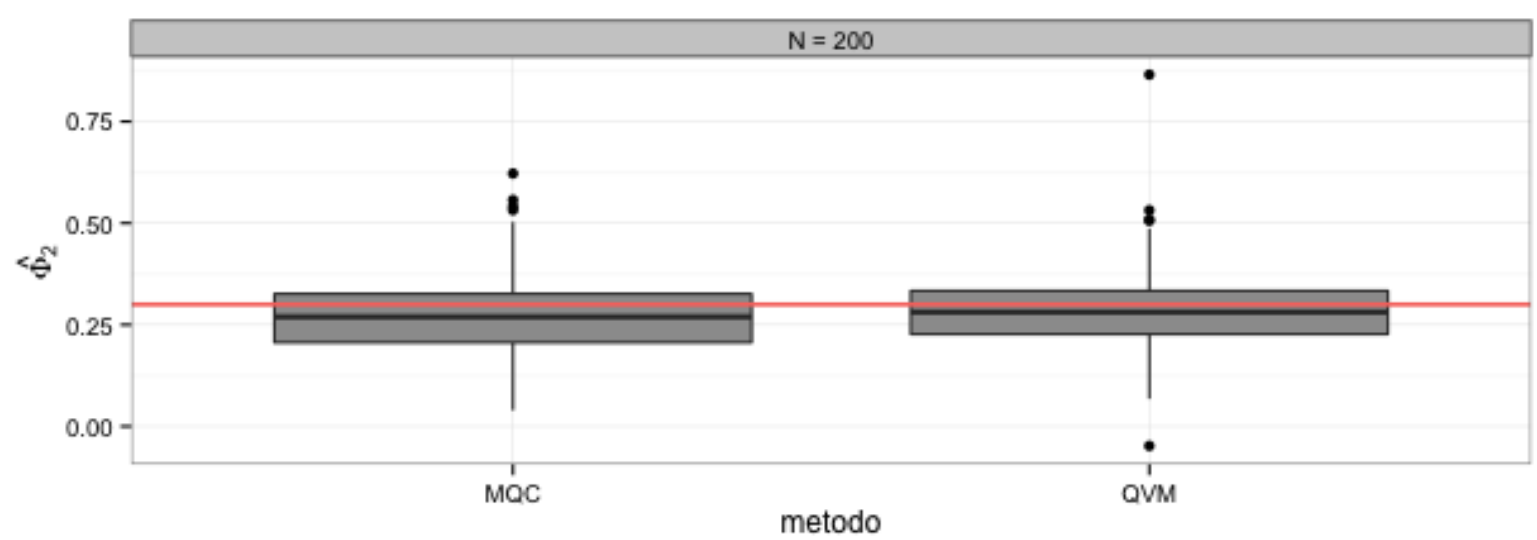

Figura 3.105: Boxplot das estimativas de $E\left[\phi_{2}^{(t)}\right]$ para o modelo gerado.

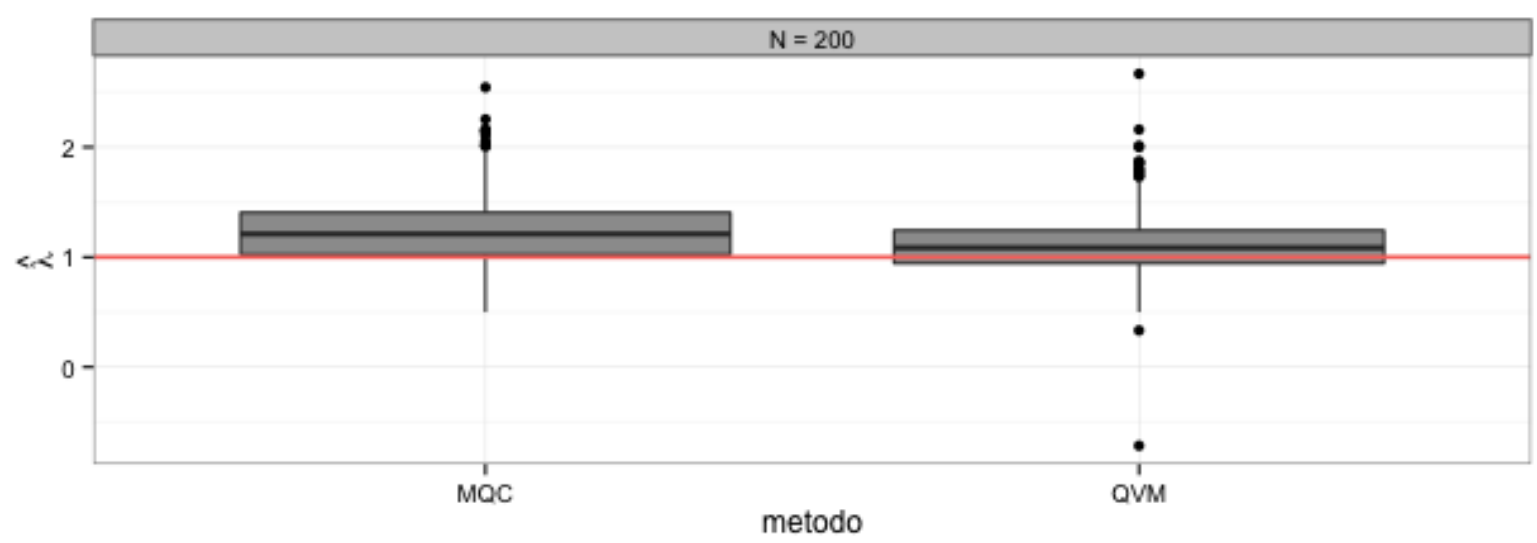

Figura 3.106: Boxplot das estimativas de $\lambda$ para o modelo gerado. 
Os histogramas alisados dos vieses de $\hat{\Phi}_{1}, \hat{\Phi}_{2}$ e $\hat{\lambda}$ são mostrados nas Figuras 3.107, 3.108 e 3.109. Os estimadores de QVM e MQC produzem vieses próximos, destacando o viés de $\hat{\lambda}$, em que o método de QVM produz estimativas melhores.

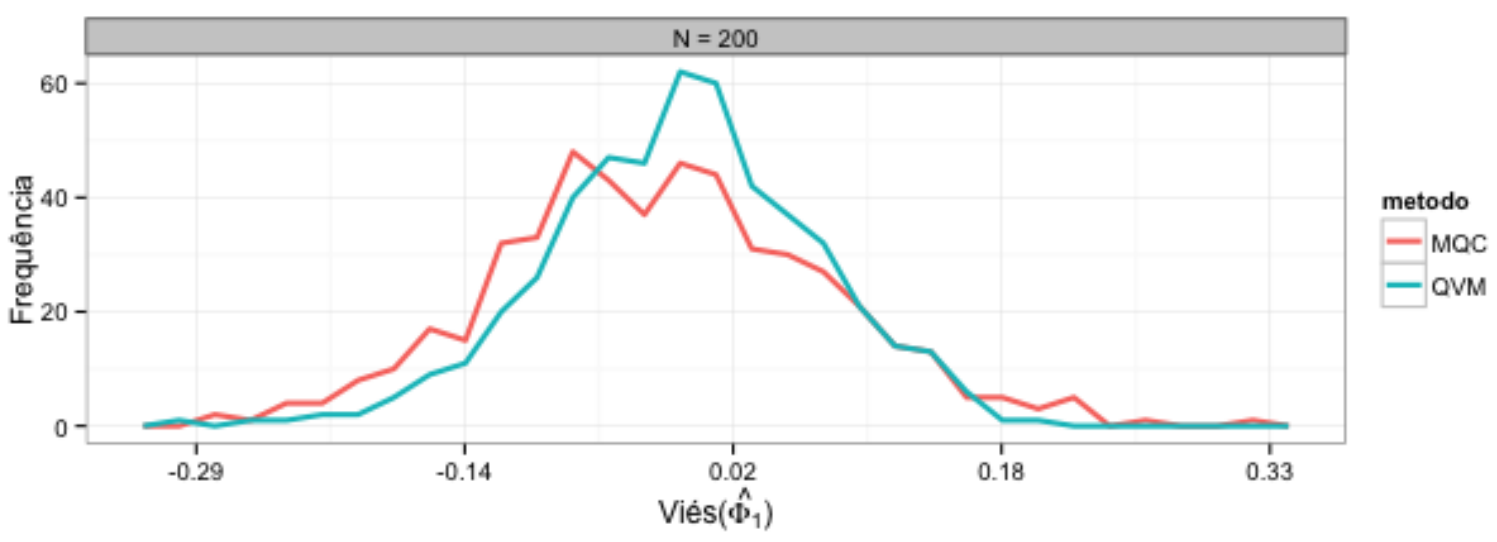

Figura 3.107: Histograma alisado do viés de $\hat{\Phi}_{1}$ para o modelo gerado.

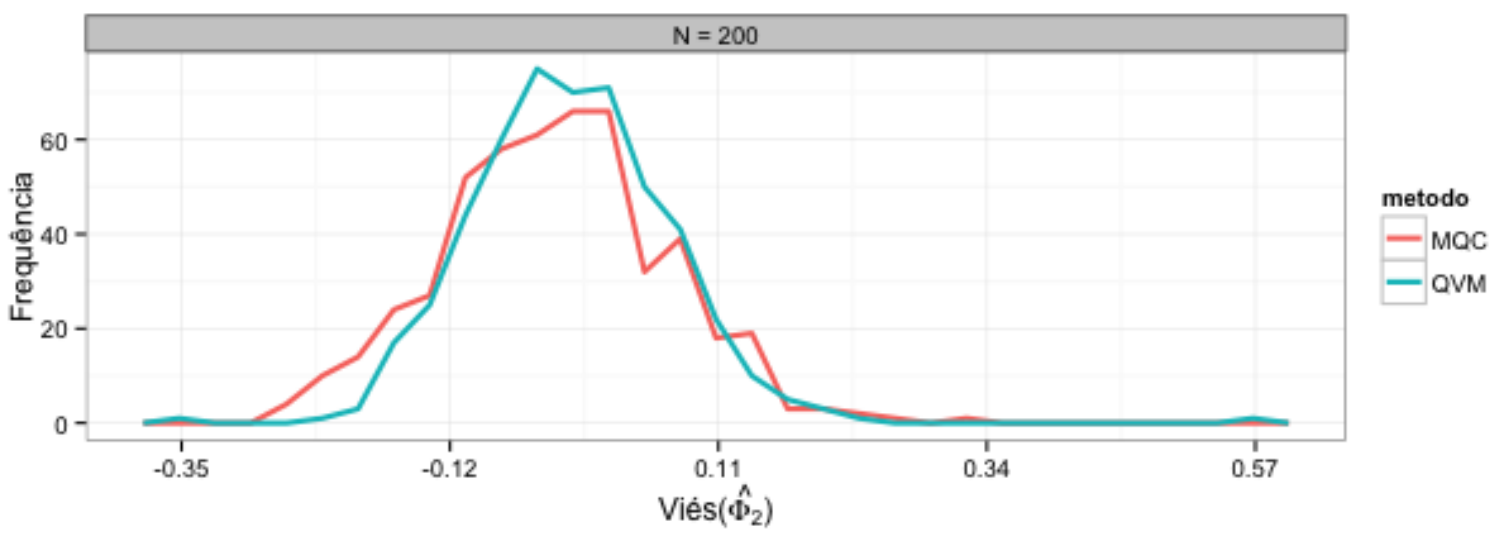

Figura 3.108: Histograma alisado do viés de $\hat{\Phi}_{2}$ para o modelo gerado.

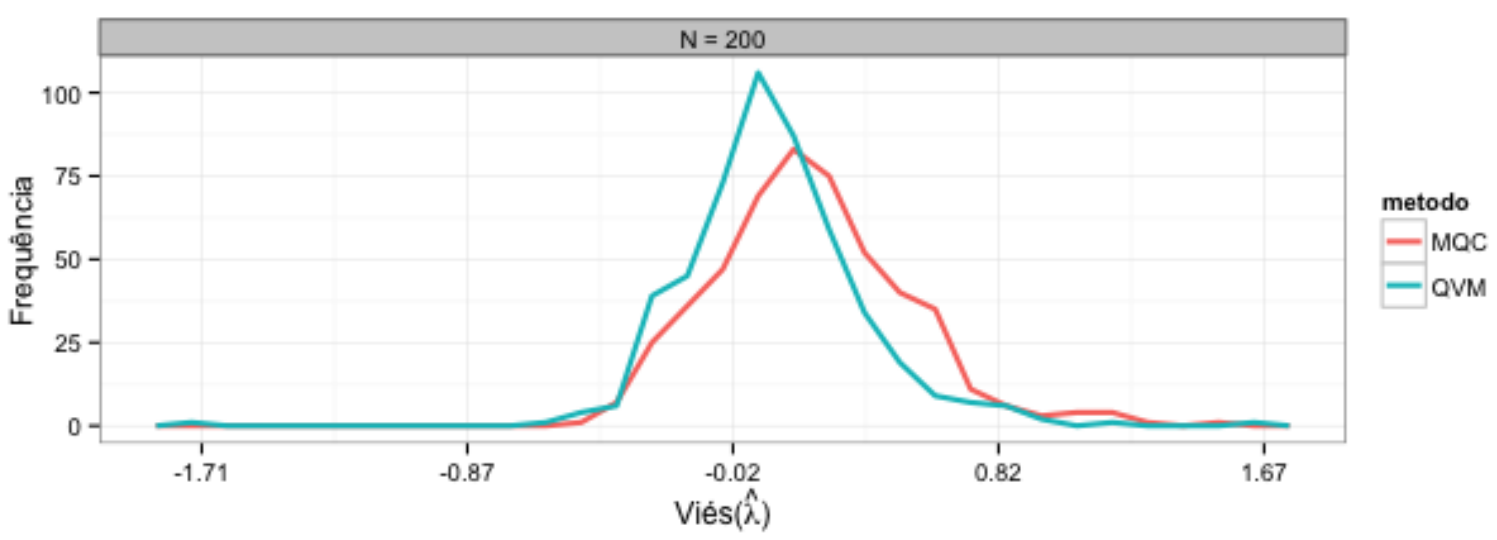

Figura 3.109: Histograma alisado do viés de $\hat{\lambda}$ para o modelo gerado. 


\begin{tabular}{|c|c|c|c|c|c|c|}
\hline & \multicolumn{3}{|c|}{ MQC } & \multicolumn{3}{|c|}{ QVM } \\
\hline & \multirow[b]{2}{*}{$\Phi_{1}$} & \multicolumn{2}{|c|}{$\Phi_{2}$} & \multirow[b]{2}{*}{$\Phi_{1}$} & \multicolumn{2}{|c|}{$\Phi_{2}$} \\
\hline & & 0,3 & 0,5 & & 0,3 & 0,5 \\
\hline \multirow{2}{*}{ Viés $\left(\hat{\Phi}_{1}\right)$} & 0,3 & -0.01784 & -0.01229 & 0,3 & -0.01097 & -0.00838 \\
\hline & 0,5 & -0.02130 & & 0,5 & -0.00944 & \\
\hline \multirow{6}{*}{ Viés $\left(\hat{\Phi}_{2}\right)$} & \multirow[b]{2}{*}{$\Phi_{1}$} & \multicolumn{2}{|c|}{$\Phi_{2}$} & \multirow[b]{2}{*}{$\Phi_{1}$} & \multicolumn{2}{|c|}{$\Phi_{2}$} \\
\hline & & 0,3 & 0,5 & & 0,3 & 0,5 \\
\hline & \multirow{3}{*}{$\begin{array}{l}0,3 \\
0,5\end{array}$} & -0.02192 & \multirow[t]{2}{*}{-0.04119} & 0,3 & -0.01364 & \multirow[t]{2}{*}{-0.02348} \\
\hline & & -0.03108 & & 0,5 & -0.01767 & \\
\hline & & \multicolumn{2}{|c|}{$\Phi_{2}$} & & \multicolumn{2}{|c|}{$\Phi_{2}$} \\
\hline & $\Phi_{1}$ & 0,3 & 0,5 & $\Phi_{1}$ & 0,3 & 0,5 \\
\hline \multirow{2}{*}{ Viés $(\hat{\lambda})$} & 0,3 & 0.09084 & \multirow[t]{2}{*}{0.21349} & 0,3 & 0.05242 & \multirow[t]{2}{*}{0.11341} \\
\hline & 0,5 & 0.22276 & & 0,5 & 0.10155 & \\
\hline
\end{tabular}

Tabela 3.11: Viés das estimativas dos parâmetros do modelo RCINAR(2) $(\lambda=1)$ baseado em 500 réplicas e $N=200$.

\begin{tabular}{|c|c|c|c|c|c|c|}
\hline & \multicolumn{3}{|c|}{ MQC } & \multicolumn{3}{|c|}{ QVM } \\
\hline & \multirow[b]{2}{*}{$\Phi_{1}$} & \multicolumn{2}{|c|}{$\Phi_{2}$} & \multirow[b]{2}{*}{$\Phi_{1}$} & \multicolumn{2}{|c|}{$\Phi_{2}$} \\
\hline \multirow{3}{*}{$\operatorname{EQM}\left(\hat{\Phi_{1}}\right)$} & & 0,3 & 0,5 & & 0,3 & 0,5 \\
\hline & 0,3 & 0.00721 & 0.00760 & 0,3 & 0.00601 & 0.00531 \\
\hline & 0,5 & 0.00949 & & 0,5 & 0.00594 & \\
\hline \multirow{6}{*}{$\operatorname{EQM}\left(\hat{\Phi_{2}}\right)$} & \multirow[b]{2}{*}{$\Phi_{1}$} & \multicolumn{2}{|c|}{$\Phi_{2}$} & \multirow[b]{2}{*}{$\Phi_{1}$} & \multicolumn{2}{|c|}{$\Phi_{2}$} \\
\hline & & 0,3 & 0,5 & & \multicolumn{2}{|r|}{0,5} \\
\hline & 0,3 & 0.00772 & 0.00835 & 0,3 & 0.00597 & 0.00549 \\
\hline & 0,5 & 0.00978 & & 0,5 & 0.00730 & \\
\hline & & \multicolumn{2}{|c|}{$\Phi_{2}$} & & \multicolumn{2}{|c|}{$\Phi_{2}$} \\
\hline & $\Phi_{1}$ & 0,3 & 0,5 & $\Phi_{1}$ & 0,3 & 0,5 \\
\hline \multirow{2}{*}{$\operatorname{EQM}(\hat{\lambda})$} & 0,3 & 0.0556 & 0.1587 & 0,3 & 0.0405 & 0.1004 \\
\hline & 0,5 & 0.1434 & & 0,5 & 0.0900 & \\
\hline
\end{tabular}

Tabela 3.12: EQM das estimativas dos parâmetros do modelo $R C I N A R(2)(\lambda=1)$ baseado em 500 réplicas e $N=200$.

Os resultados apresentados na Tabela 3.11 mostram que mais uma vez o estimador de QVM produz estimativas mais assertivas, comparado ao estimador de MQC. É possível verificar que o viés médio das estimativas dadas por QVM assumem a metade do valor do viés médio dado pelo estimador de MQC. A Tabela 3.12 mostra os EQM dos modelos simulados. Nota-se que em todas as combinações de parâmetros $\Phi_{1}$ e $\Phi_{2}$ os valores do EQM obtidos pelo estimador MQC superam os mesmos dados por QVM. 


\subsection{Previsão}

A análise de séries temporais possui diferentes objetivos de acordo com o foco do projeto ou a pergunta que se deseja responder. Um dos principais intuitos desse estudo é a antecipação aos movimentos da série, a previsão dos próximos valores. Uma vez estipulado a estrutura do modelo que gera o processo $\left\{X_{t}\right\}_{t \in[1, N]}$ estima-se os parâmetros e segue-se com o objetivo de previsão da estrutura já completamente determinada.

Segundo Jin-Guan e Li-Yuan (1991) podemos definir o preditor de mínima variância $\hat{X}_{N}(h)$ de $X_{N+h}$ como uma esperança condicionada à informação passada.

$$
\begin{aligned}
\hat{X}_{N}(h) & =E\left(X_{N+h} / F_{N}\right) \\
& =\sum_{j=1}^{p} E\left(\alpha_{j} \circ X_{N+h-j} / F_{N}\right)+\hat{\lambda} \\
& =\sum_{j=1}^{p} \alpha_{j} E\left(X_{N+h-j} / F_{N}\right)+\hat{\lambda} \\
& =\sum_{j=1}^{p} \alpha_{j} \hat{X}_{N}(h-j)+\hat{\lambda} .
\end{aligned}
$$

Considerando $F_{N}$ toda a informação probabilística do conjunto $\left\{X_{1}, X_{2}, \cdots, X_{N}\right\}$. Nota-se de (3.31) que a previsão do modelo INAR(p) possui representação muito próxima do modelo $\mathrm{AR}(\mathrm{p})$.

Assim como definido em Jin-Guan e Li-Yuan (1991) para previsões de modelos INAR, pode-se extender a maneira de realizar previsões para os modelos RCINAR. Para isso utilizamos a seguinte propriedade de esperança, $E[A]=E[E[A / B]]$.

Seja $\Phi_{j}=E\left[\phi_{j}^{(t)}\right]$ e $\left\{X_{t}\right\}_{t \in[1, N]}$ um processo $\operatorname{RCINAR}(\mathrm{p})$, temos:

$$
\begin{aligned}
\hat{X}_{N}(h) & =E\left(X_{N+h} / F_{N}\right) \\
& =\sum_{j=1}^{p} E\left(\phi_{j}^{(t)} \circ X_{N+h-j} / F_{N}\right)+\hat{\lambda} \\
& =\sum_{j=1}^{p} \Phi_{j} E\left(X_{N+h-j} / F_{N}\right)+\hat{\lambda} \\
& =\sum_{j=1}^{p} \Phi_{j} \hat{X}_{N}(h-j)+\hat{\lambda} .
\end{aligned}
$$




\section{Capítulo 4}

\section{Aplicação}

Nesse capítulo aplicaremos as metodologias estudadas em dados reais. Tanto o INAR quanto o RCINAR são ajustados através da estimação dada por máxima verossimilhança e quase verossimilhança modificada, respectivamente. Foram estudados dois casos para aplicação, ambos tratam do mercado de crédito, mas com óticas distintas. Abaixo, segue os problemas estudados:

1. Número de empresas com falência decretada

2. Demanda por crédito - número de consultas à base de dados da Serasa Experian

O procedimento de avaliação das séries é realizado de acordo com os passos sugeridos por Box et al. (2008): examina-se a série em relação às suas características de dependência linear; estima-se com um dos modelos propostos; ajusta-se o comportamento dos resíduos e calculam-se previsões.

\subsection{Dados de falência}

Existem muitas definições para falência; no campo econômico acredita-se que a falência ocorre quando a empresa não é mais capaz de quitar as dívidas com seus credores. Essa definição é conhecida por muitos como estado de insolvência. Já a definição jurídica de falência é que além de observar o estado de insolvência, deve-se executar seu patrimônio. A falência sob a ótica jurídica é decretada por um juiz de direito, sendo chamada de falência decretada.

A série estudada tem origem no departamento de Indicadores da Serasa Exerian, podendo ser obtida de forma gratuita direto do site. Trata-se de um levantamento mensal da quantidade de empresas que solicitam falência ou possuem falência decretada, a série tem início em janeiro de 2005 e fim em dezembro de 2012 totalizando 96 observações. Esse indicador é calculado para diversos portes de empresas, nesse estudo enfatizamos as médias empresas com falência decretada, chamando-as daqui pra frente de quantidade de falências.

A Figura 4.1 apresenta a série de número de empresas de médio porte com falência decretada; essa série parece se comportar bem de acordo com as premissas do modelo RCINAR, sendo estacionária. Na Figura 4.2 temos o histograma (a) e boxplot (b) da série de quantidade de falências; nota-se por esses gráficos que a quantidade de empresas médias com falência decretada está em cerca de 5 por mês, não ultrapassando 15 historicamente. 


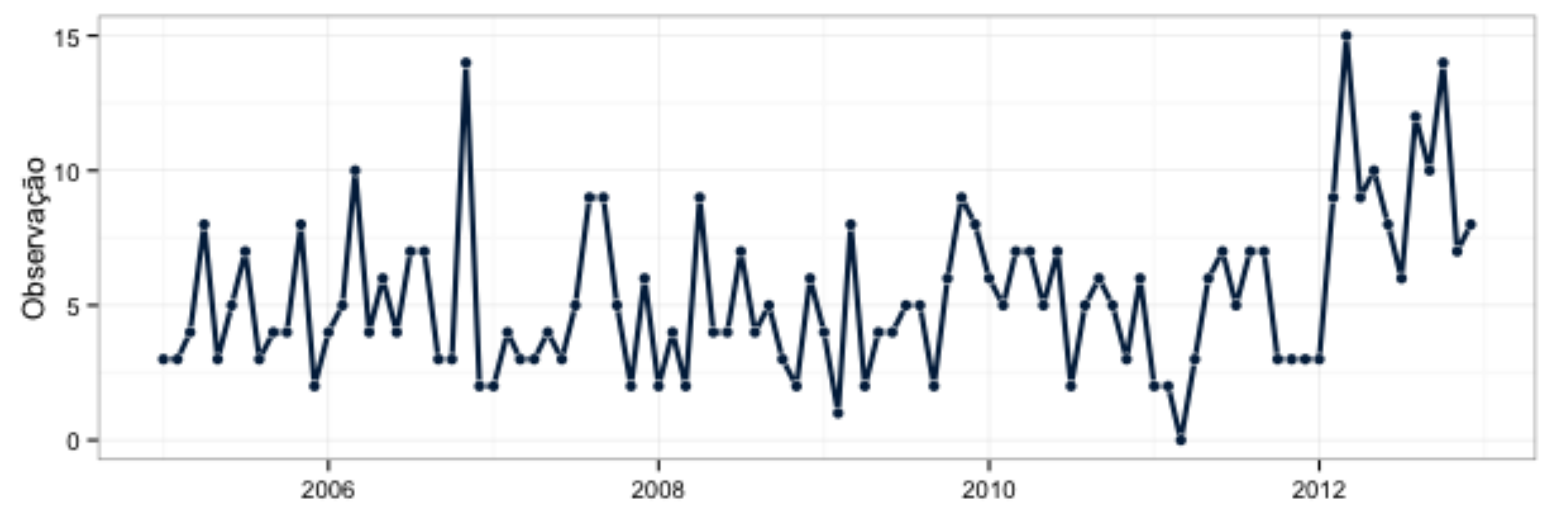

Figura 4.1: Número de falências decretadas - Médias empresas.

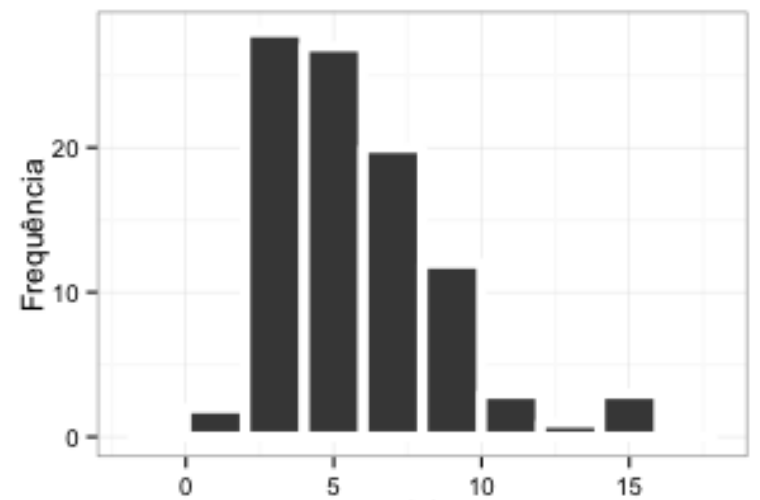

(a)

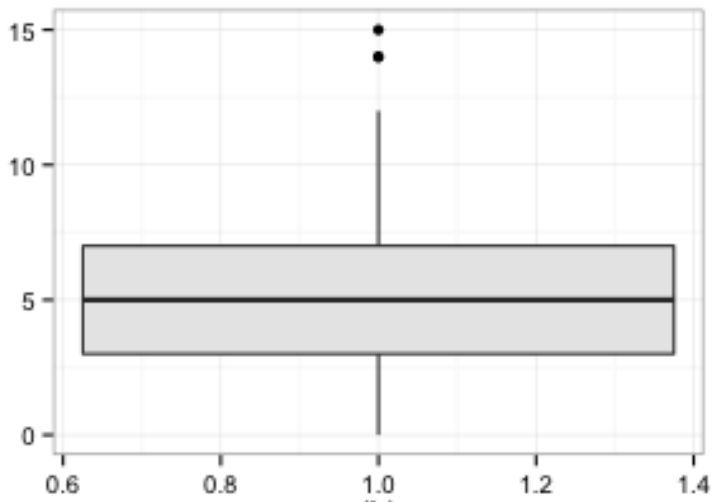

(b)

Figura 4.2: Histograma e boxplot do número de falência decretada-Médias empresas.
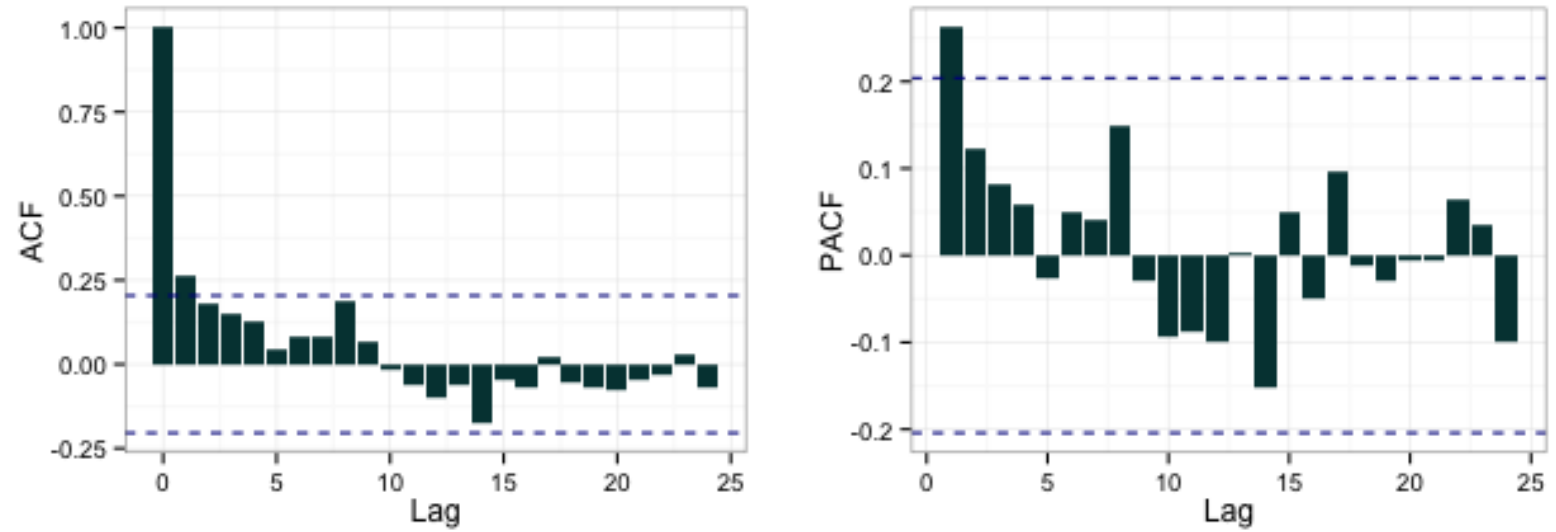

Figura 4.3: Autocorrelação e autocorrelação parcial do número de falência decretada - Médias empresas.

Considerando a série de quantidade de falências estacionária, podemos analisar a autocorrelação e autocorrelação parcial para identificar que tipo de estrutura de dependência existe. A Figura 4.3 mostra que um modelo de ordem um pode ser adotado para ajustar a série. Seguindo essa afirmação e utilizando os estimadores de quase verossimilhança modificado (QVM) proposto é possivel estimar os parâmetros. Seguindo a notação de (3.22), o modelo proposto para os dados apresentados é:

$$
X_{t}=\phi_{1}^{(t)} \circ X_{t-1}+I_{t}
$$

em que os valores estimados para $E\left[\phi_{1}^{(t)}\right]$ e $\lambda$ são 0,2536 e 3,978 respectivamente. 


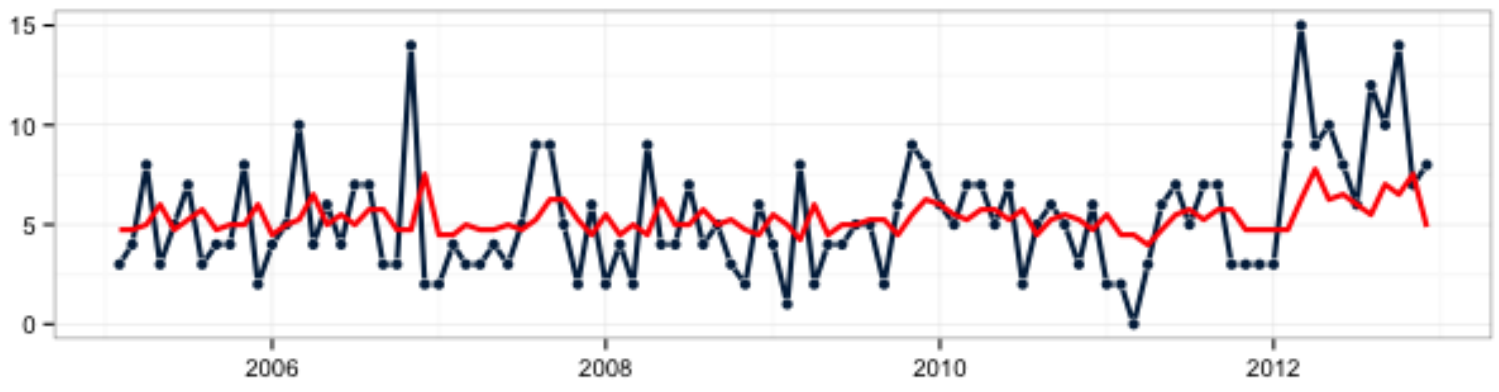

Figura 4.4: Série real e ajuste do modelo RCINAR(1).

A série real e a ajustada são graficadas na Figura 4.4, vemos que o ajuste é capaz de acompanhar a tendência da série real. A Figura 4.5 mostra o histograma e boxplot dos resíduos do modelo ajustado; notamos a simetria dos mesmos cerca de zero.

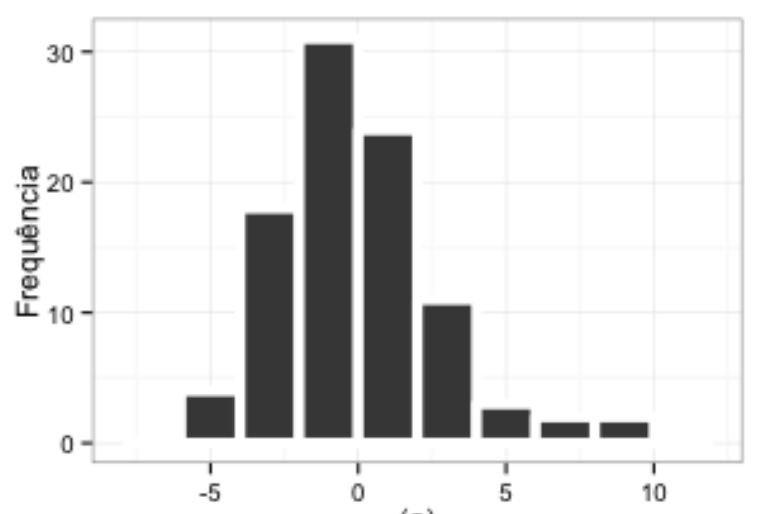

(a)

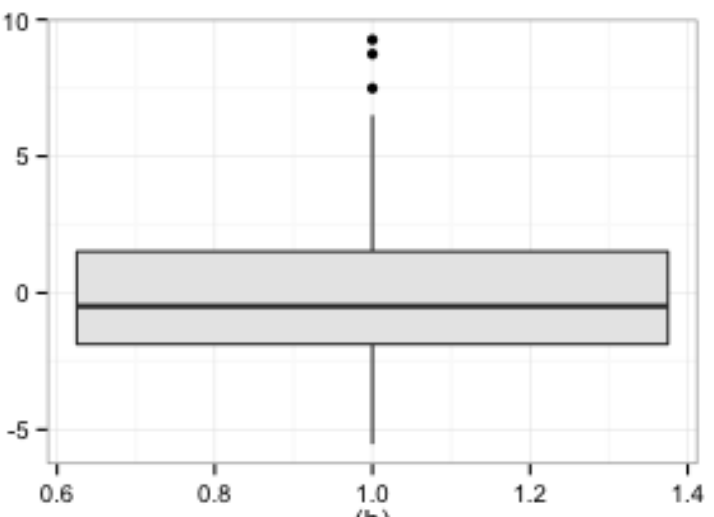

(b)

Figura 4.5: Histograma e boxplot do resíduo do ajuste.
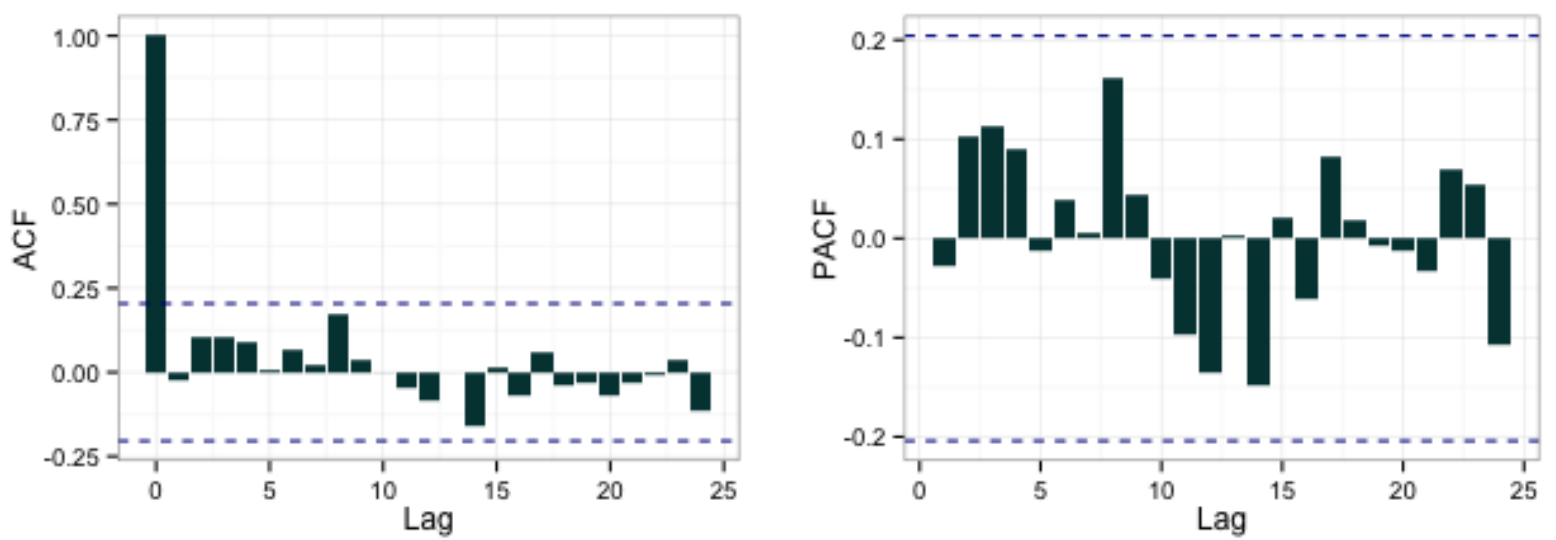

Figura 4.6: Autocorrelação e autocorrelação parcial do resíduo do ajuste. 


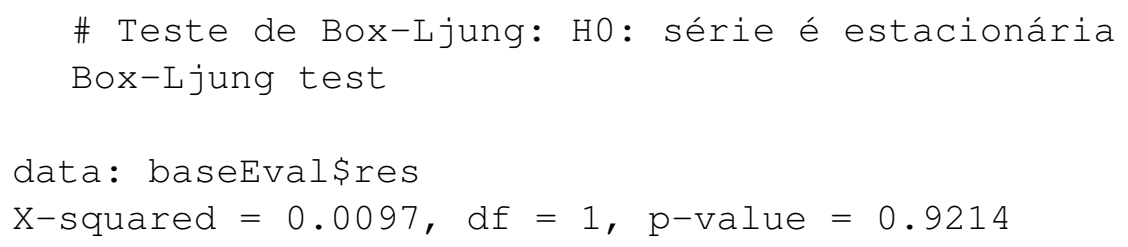

O diagnóstico é finalizado com a avaliação da autocorrelação e autocorrelação parcial do resíduo mostrados na Figura 4.6 e com o teste de Box-Ljung no quadro acima. Notamos que o resíduo não possui ACF ou PACF significantes, mostradas na Figura 4.6, assim também concluímos no teste de Box-Ljung, que não rejeitamos a hipótese de que a série é estacionária.

A aplicação está completa com o cálculo da previsão. O modelo foi ajustado com a série sem os dois últimos registros, esses pontos foram previstos e estão apresentados na Tabela 4.1, juntamente com o erro de previsão observado. O MAE é calculado com valor de 1,82.

\begin{tabular}{cccc}
\hline \hline Data & Observado & Previsão & Erro \\
\hline Nov-2012 & 7 & 7,5283 & -0.5283 \\
Dez-2012 & 8 & 4,8782 & 3,1218 \\
\hline \hline
\end{tabular}

Tabela 4.1: Comparativo da previsão. 


\subsection{Dados de crédito}

Ao comprar algum produto, realizar um financiamento ou simplesmente pedir um empréstimo, em geral a pessoa é consultada num bureau de crédito como a Serasa Experian; essa consulta gera um registro na base de dados. Cada registro pode ser contabilizado e tomado como indicador da demanda por crédito; esse indicador possui um significado quando se fala em modelos de risco de crédito.

Em geral quanto maior a quantidade da demanda por crédito num curto período de tempo, entende-se que esse consumidor pode não honrar com seus deveres financeiros e tende a ser um consumidor com propensão a ser inadimplente.

O estudo efetuado nessa aplicação tratou uma base com quase trinta mil empresas. Em cada uma delas avaliou-se a quantidade de consultas mensais desde a primeira data com demanda por crédito. A intenção é avaliar se o comportamento da série de consulta nos traz alguma variável preditiva para segmentar as empresas em relação ao seu risco associado. Das trinta mil empresas, extraímos cerca de oito mil delas em que a dependência linear (ACF e PACF) indicava o comportamento de um $\operatorname{RCINAR}(2)$.

Essa seção traz uma das oito mil empresas que ajustamos um modelo INAR e RCINAR, de ordem adequada ao comportamento da série observada,. A Figura 4.7 mostra a quantidade de consultas que essa empresa teve no bureau da Serasa Experian; em seguida temos na Figura 4.8 o histograma e boxplot da série.

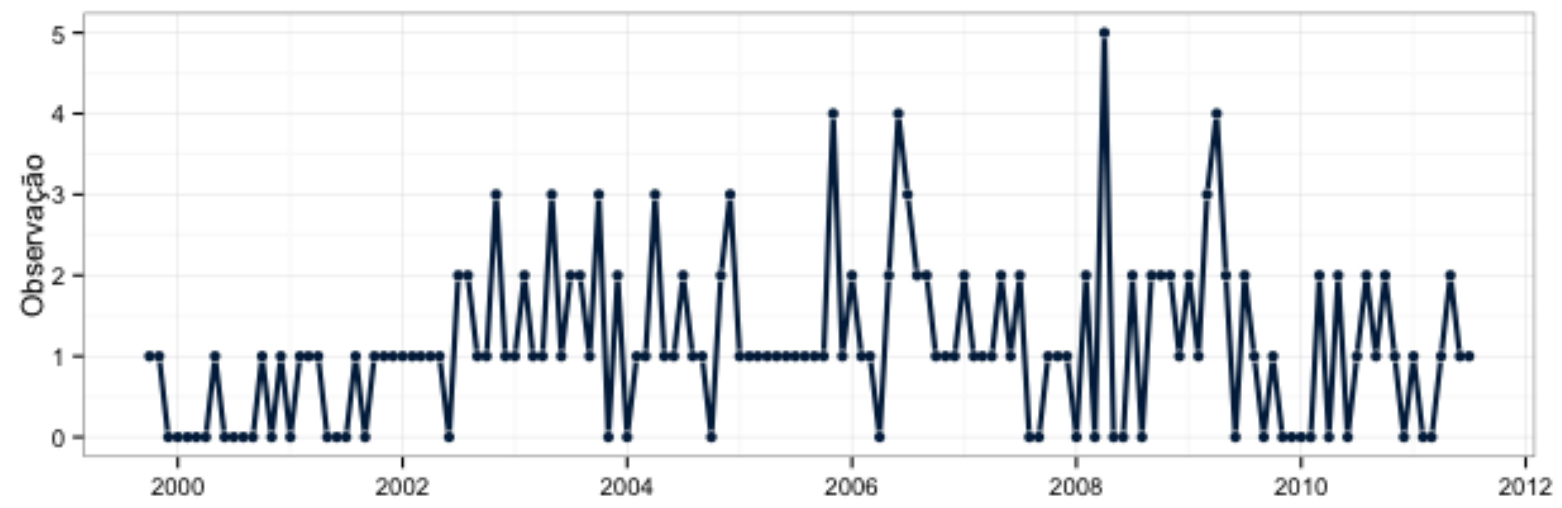

Figura 4.7: Número de consultas.

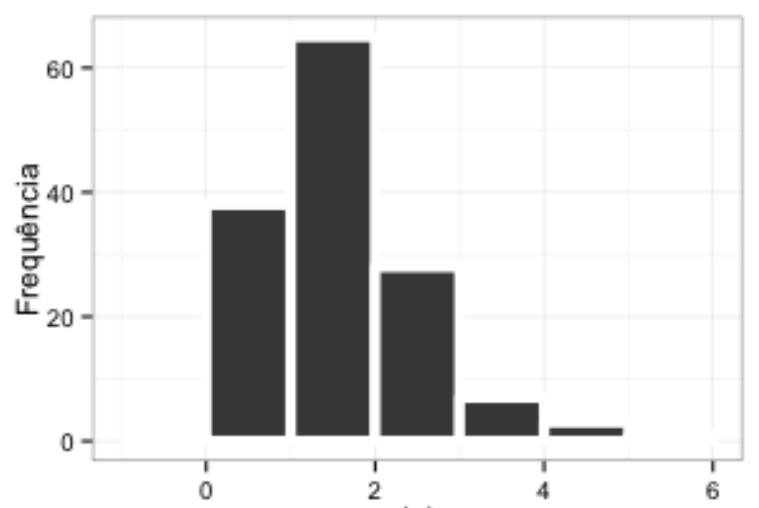

(a)

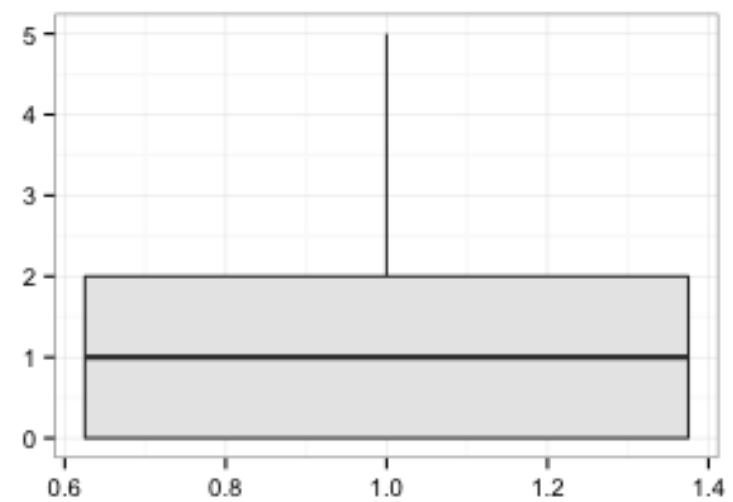

(b)

Figura 4.8: Histograma e boxplot da quantidade de consultas. 

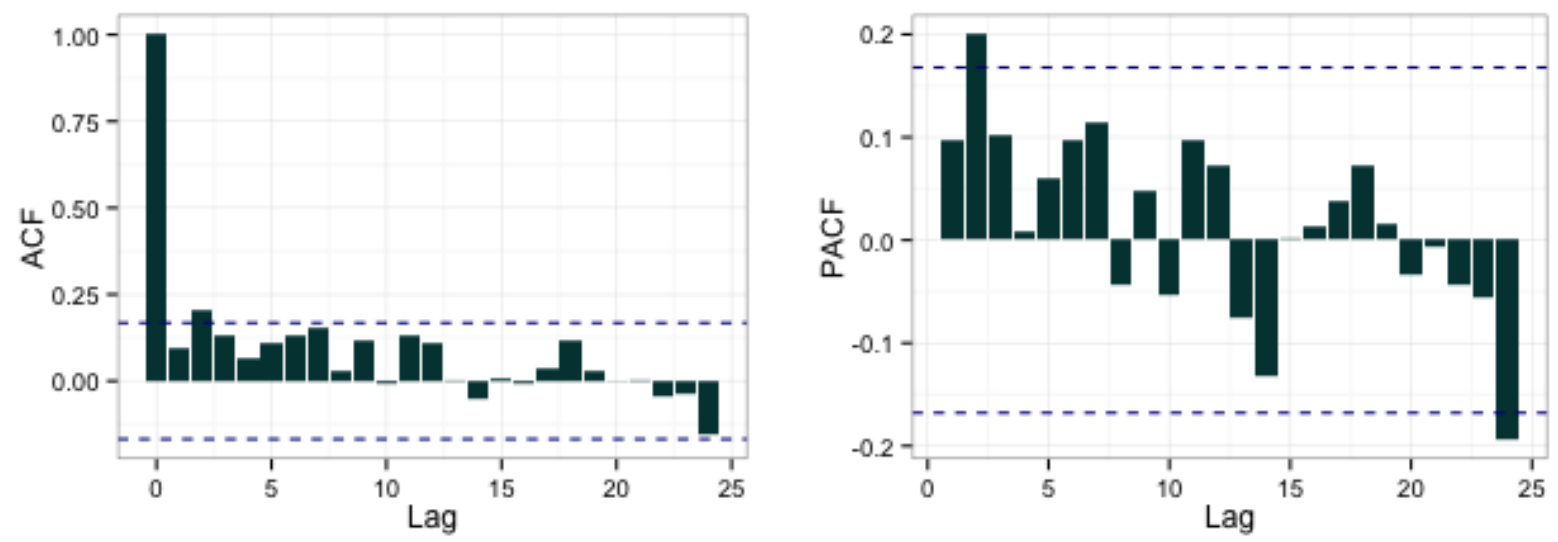

Figura 4.9: Autocorrelação e autocorrelação parcial da quantidade de consultas.

A série apresenta autocorrelação parcial significante de ordem dois, como mostra a Figura 4.9. Esse comportamento da série indica que o modelo RCINAR(2) pode ser utilizado para ajustar essa estrutura, tal modelo é apresentado em (4.2).

$$
X_{t}=\phi_{1}^{(t)} \circ X_{t-1}+\phi_{2}^{(t)} \circ X_{t-2}+I_{t},
$$

em que as estimativas dadas por QVM para $E\left[\phi_{1}^{(t)}\right], E\left[\phi_{2}^{(t)}\right]$ e $\lambda$ são $0,1073,0,23$ e 0,75 respectivamente.

A avaliação da qualidade do ajuste do modelo segue com o gráfico da quantidade de consultas observada versus ajuste dado pelo modelo mostrado na Figura 4.10. Seguindo a análise temos o histograma e boxplot do resíduo dados na Figura 4.11. Mais uma vez, o histograma e o boxplot mostram os resíduos centrados em zero.

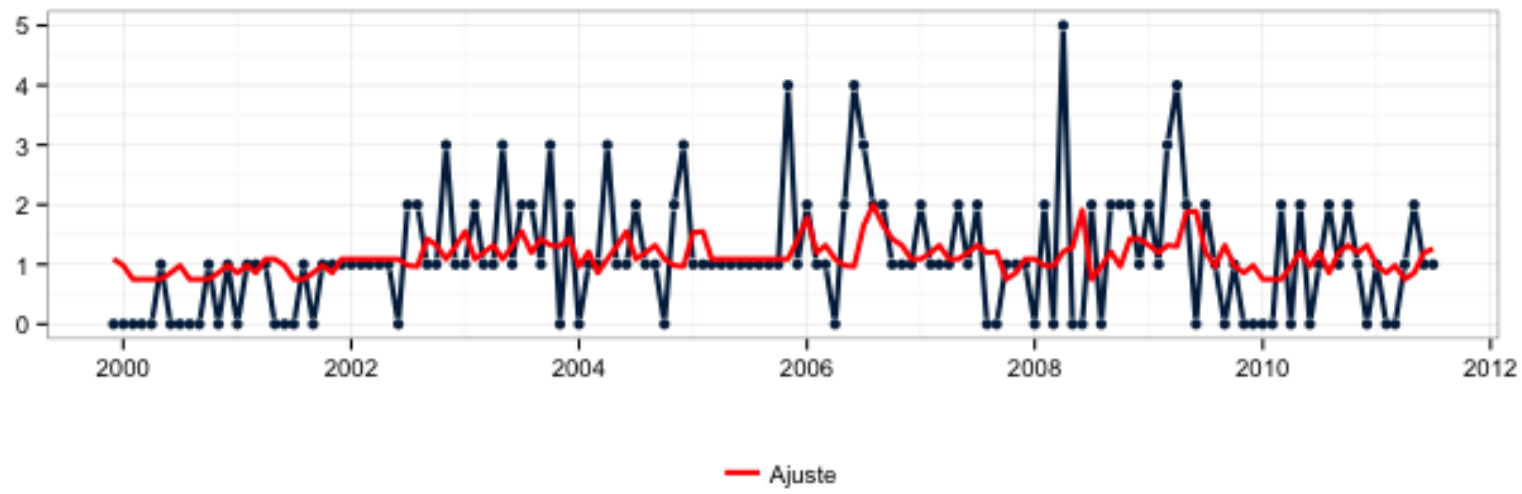

Figura 4.10: Série real e ajuste do modelo RCINAR(2). 


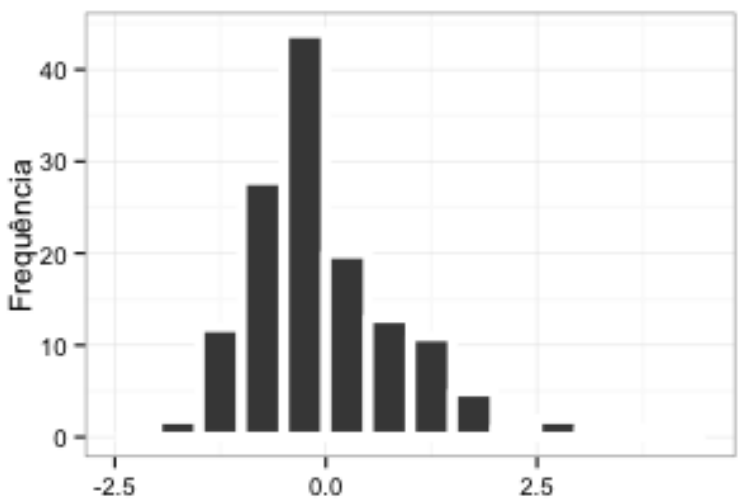

(a)

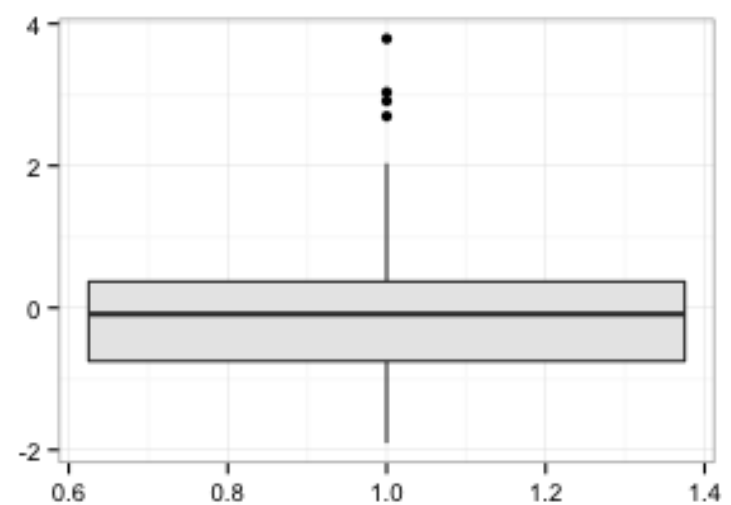

(b)

Figura 4.11: Histograma e boxplot do resíduo do ajuste.
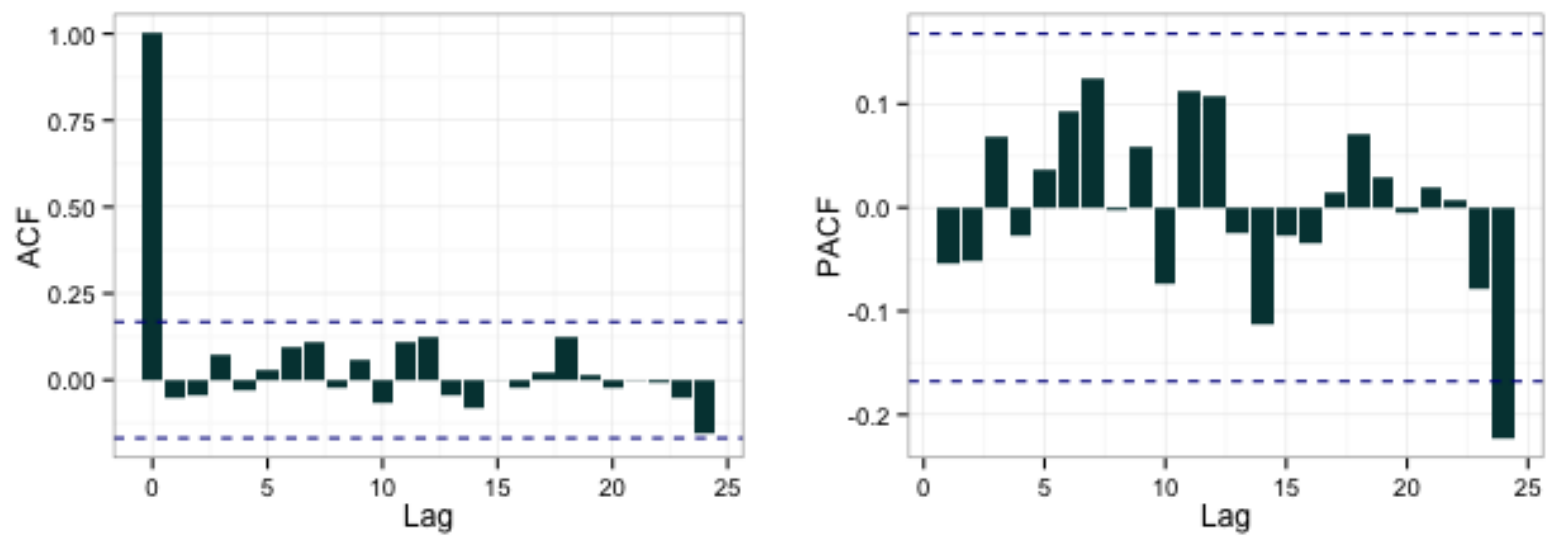

Figura 4.12: Autocorrelação e autocorrelação parcial do resíduo do ajuste.

A análise de autocorrelação e autocorrelação parcial são dados na Figura 4.12, nota-se que o modelo estimado por quase verossimilhança modificada é adequado. O teste de Box-Ljung é realizado para reforçar essa afirmação.

\# Teste de Box-Ljung: HO: série é estacionária

Box-Ljung test

data: baseEval\$res

$\mathrm{x}$-squared $=0.2756$, $\mathrm{df}=1, \mathrm{p}$-value $=0.5996$

Diferente do objetivo para a série de falência, a aplicação para crédito busca entender o comportamento temporal das séries de demanda por crédito de cada empresa. Após o ajuste de modelos INAR e RCINAR à série de quantidade de consultas, temos as estimativas $\hat{\Phi}_{1}$, $\hat{\Phi}_{2}$ e $\hat{\lambda}$ para cada empresa. Essas estimativas são o produto final da aplicação, pois condensam toda a informação histórica de demanda por crédito de cada empresa, assim as estimativas serão tratadas como variáveis em um modelo de risco de crédito. 


\section{Capítulo 5}

\section{Conclusões}

A conclusão desse estudo é dividido em duas partes, a primeira tratada sob a ótica metodológica e a segunda sob a aplicação.

Vimos que em ambos modelos, INAR e RCINAR, existem distintas metodologias de estimação, o estudo de simulação trouxe a comparação de cada uma delas. Para o modelo INAR a maior eficiência do método de máxima verossimilhança ficou clara, principalmente quando extendemos o modelo INAR para ordem dois. Vimos que os estimadores de YW e MQC produzem estimativas viesadas quando simulando processos de ordem dois, enquanto que as estimativas dadas por MVC são centradas no verdadeiro valor do parâmetro. O mesmo ocorreu quando avaliamos os modelos RCINAR; nesse caso os estimadores dados por QVM apresentaram leve vantagem sobre os de MQC, para todas as combinações de parâmetros estudadas.

Baseado nesses estudos, definimos como estimador principal o estimador de MVC para modelos INAR e QVM para modelos RCINAR para ajustamentos de dados reais.

As aplicações dos modelos RCINAR foram bastante satisfatórias principalmente para os dados de crédito, pois essa metodologia traz uma visão sobre a série de quantidade de consulta que não existia no mercado. A aplicação do modelo RCINAR nas séries de consultas foram extendidas para diferentes ordens de dependências $(\mathrm{ACF} / \mathrm{PACF})$ observada nas séries. O resultado foi a criação de novas variáveis para distinguir boas e más empresas na visão de crédito.

\subsection{Sugestões para Pesquisas Futuras}

O estudo de modelos RCINAR se mostrou bastante promissor no âmbito de variáveis para modelos de risco de crédito. A aplicação desse modelo com diferentes ordens em séries de quantidade de consultas é uma sugestão para outros estudos.

Existem modelos que devem ser estudados, como modelos com o coeficiente determinado por variáveis exógenas e modelos em que a taxa de inovação $\lambda$ seja variante no tempo. 
CONCLUSÕES 


\section{Apêndice A}

\section{Código implementado}

Os códigos para gerar os processos INAR e RCINAR estão espostos nessa sessão, assim como o programa dos estimadores. Todos implementados em R.

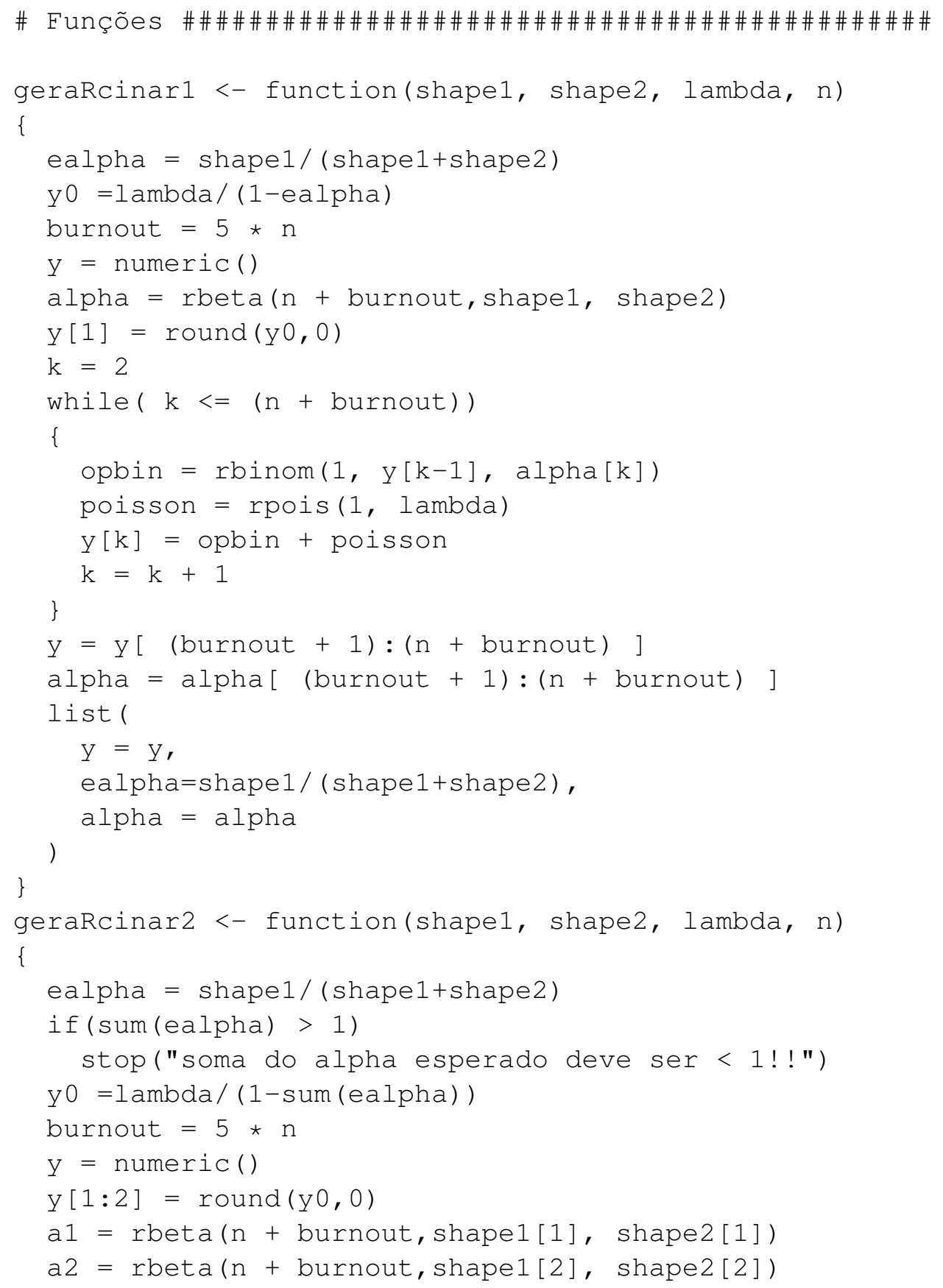




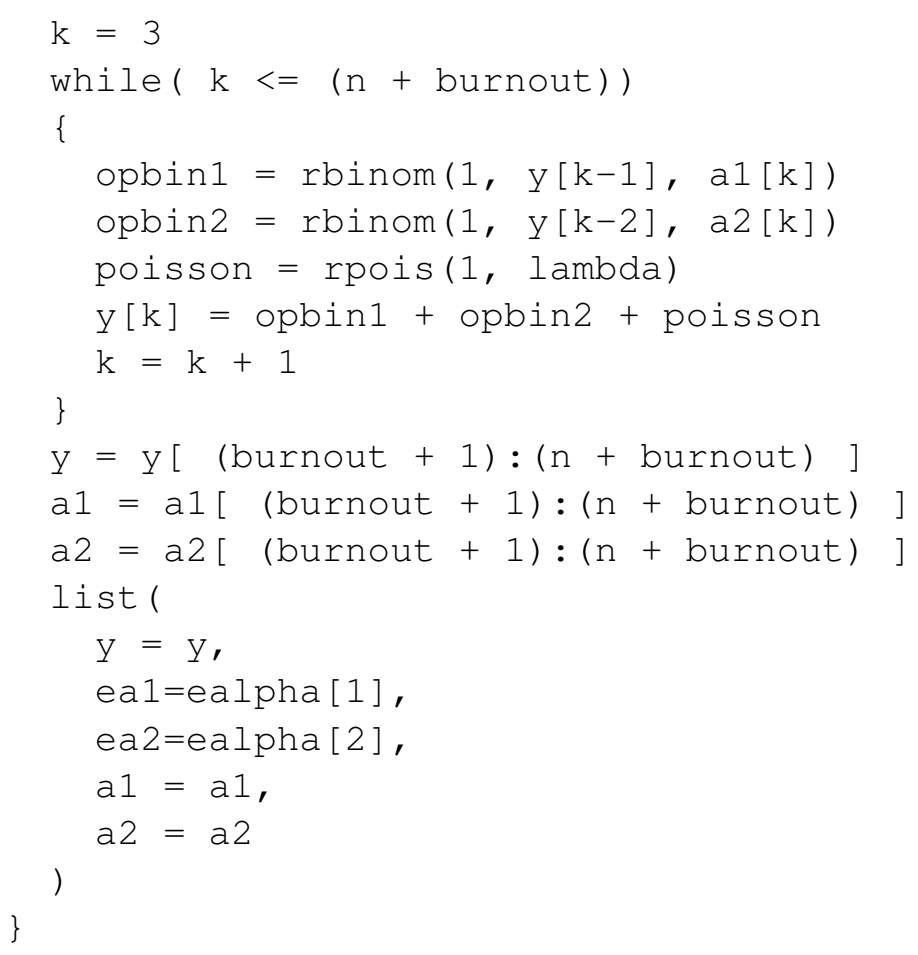




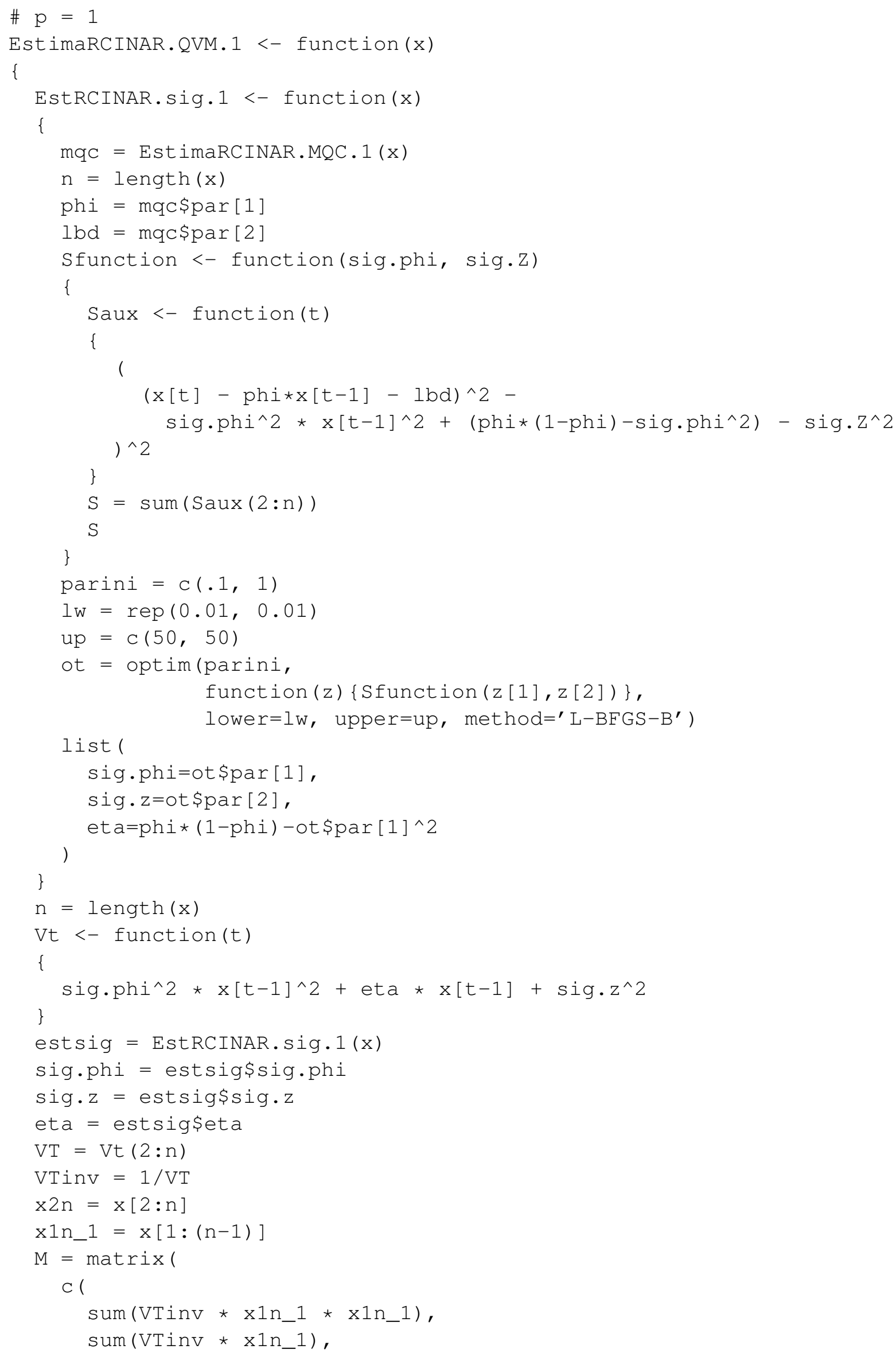




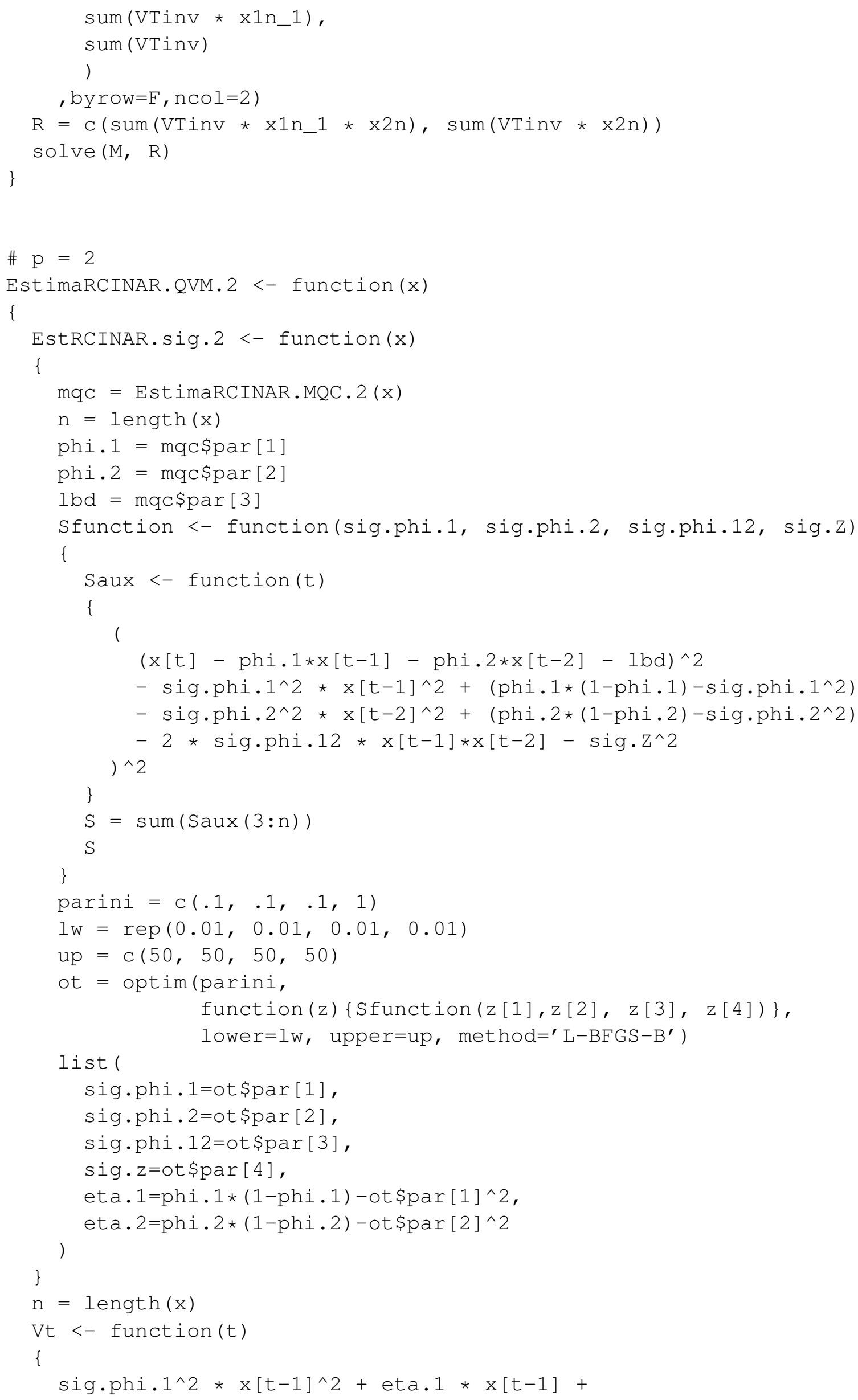




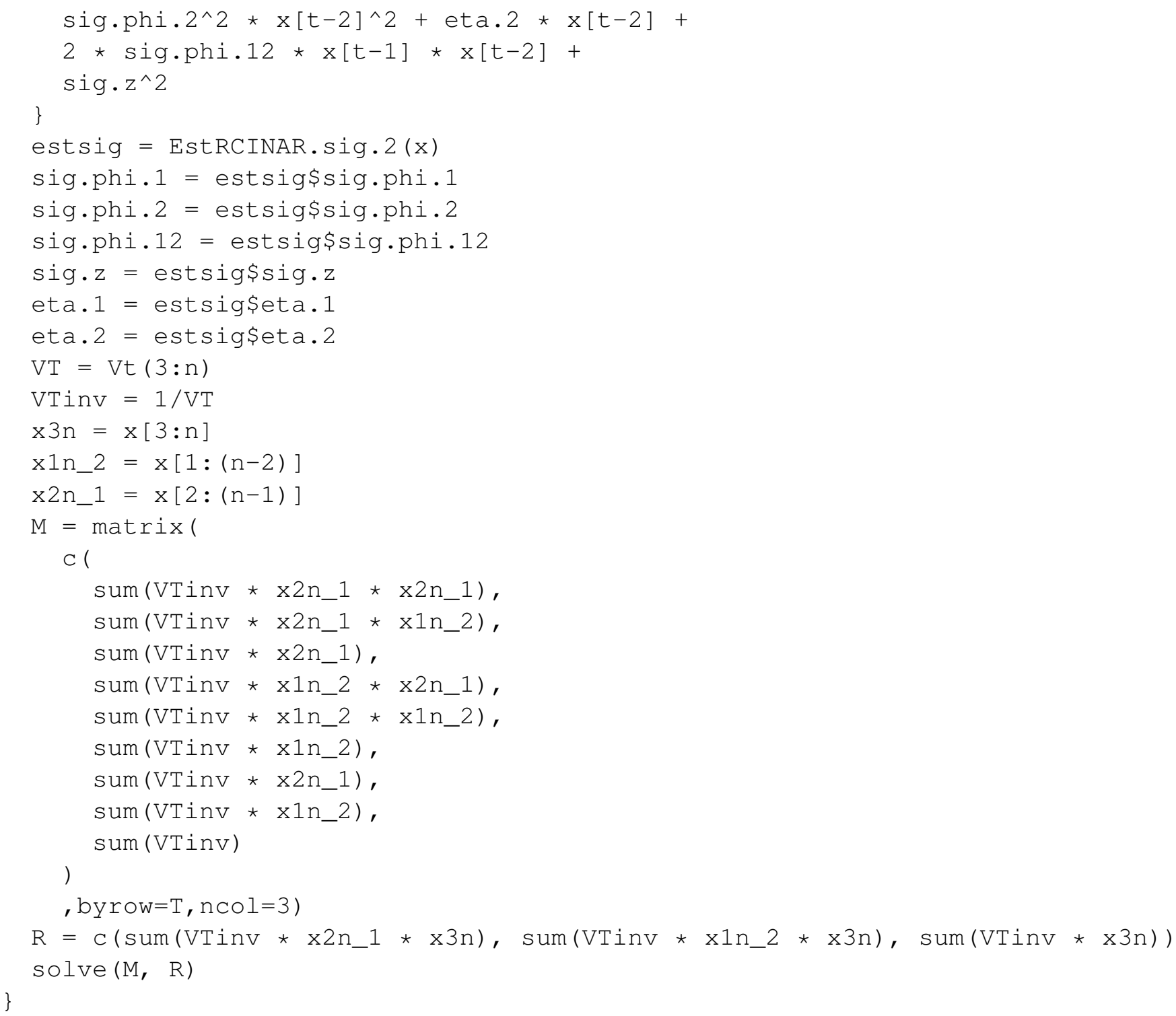




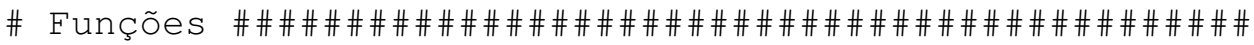

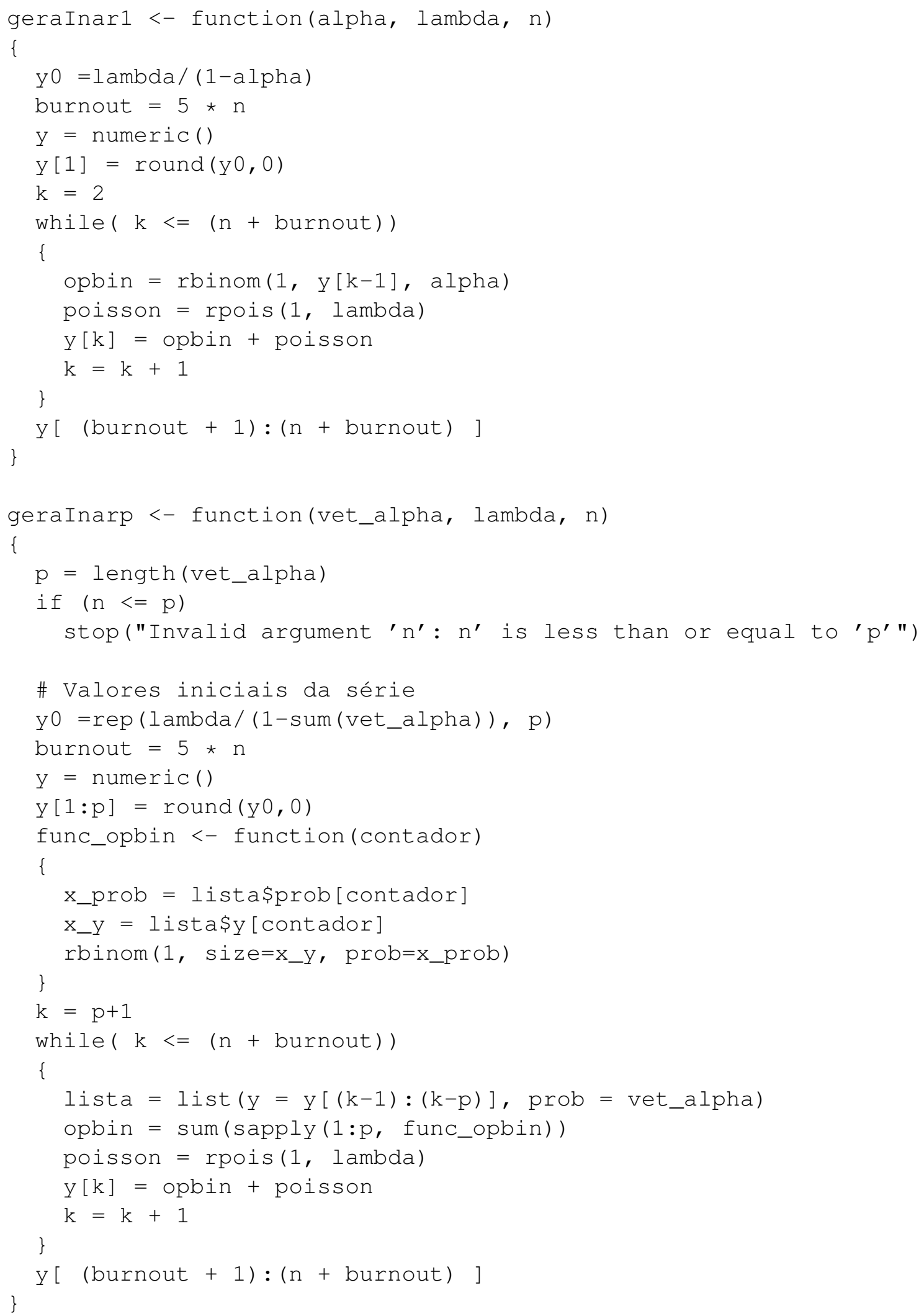




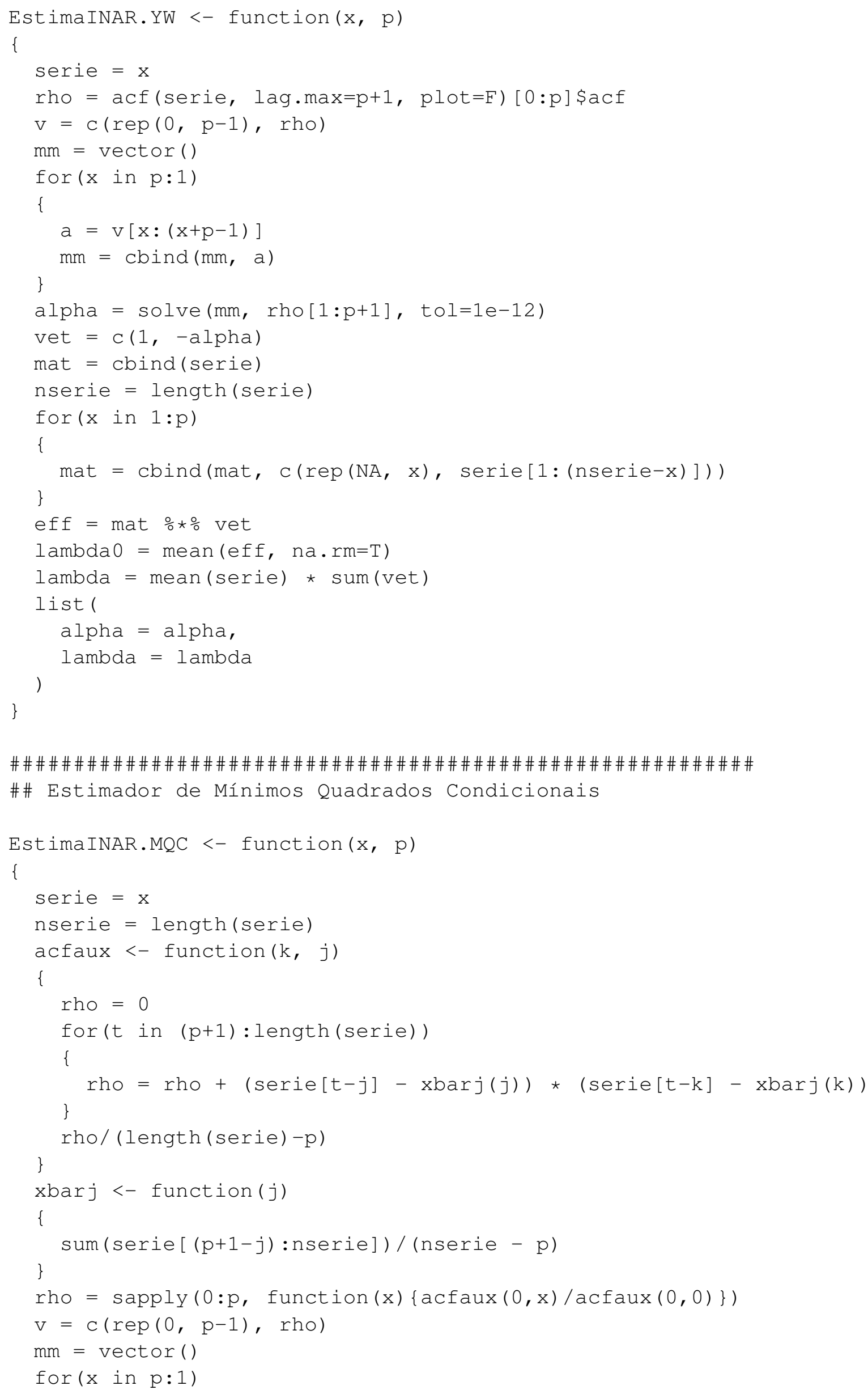




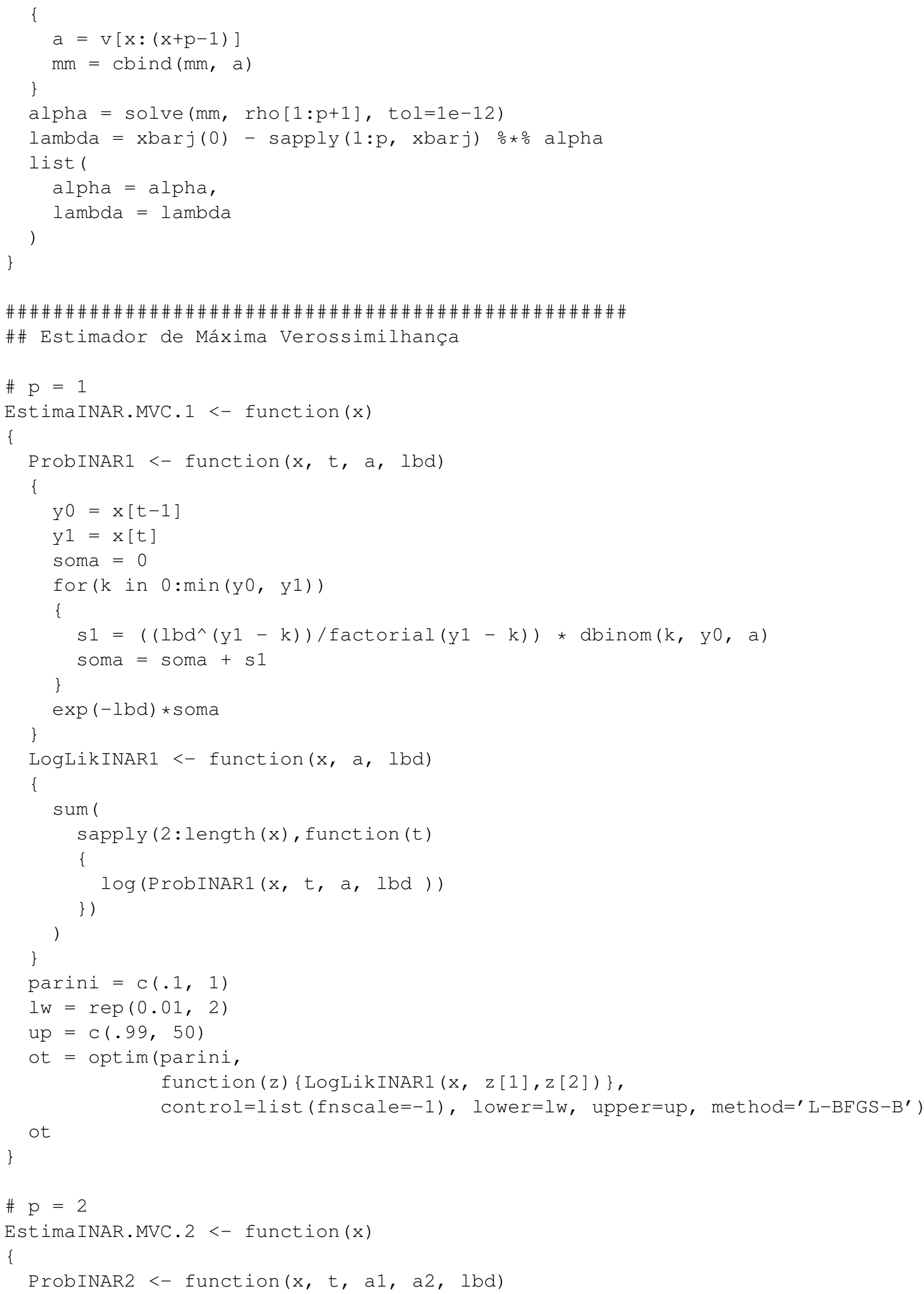




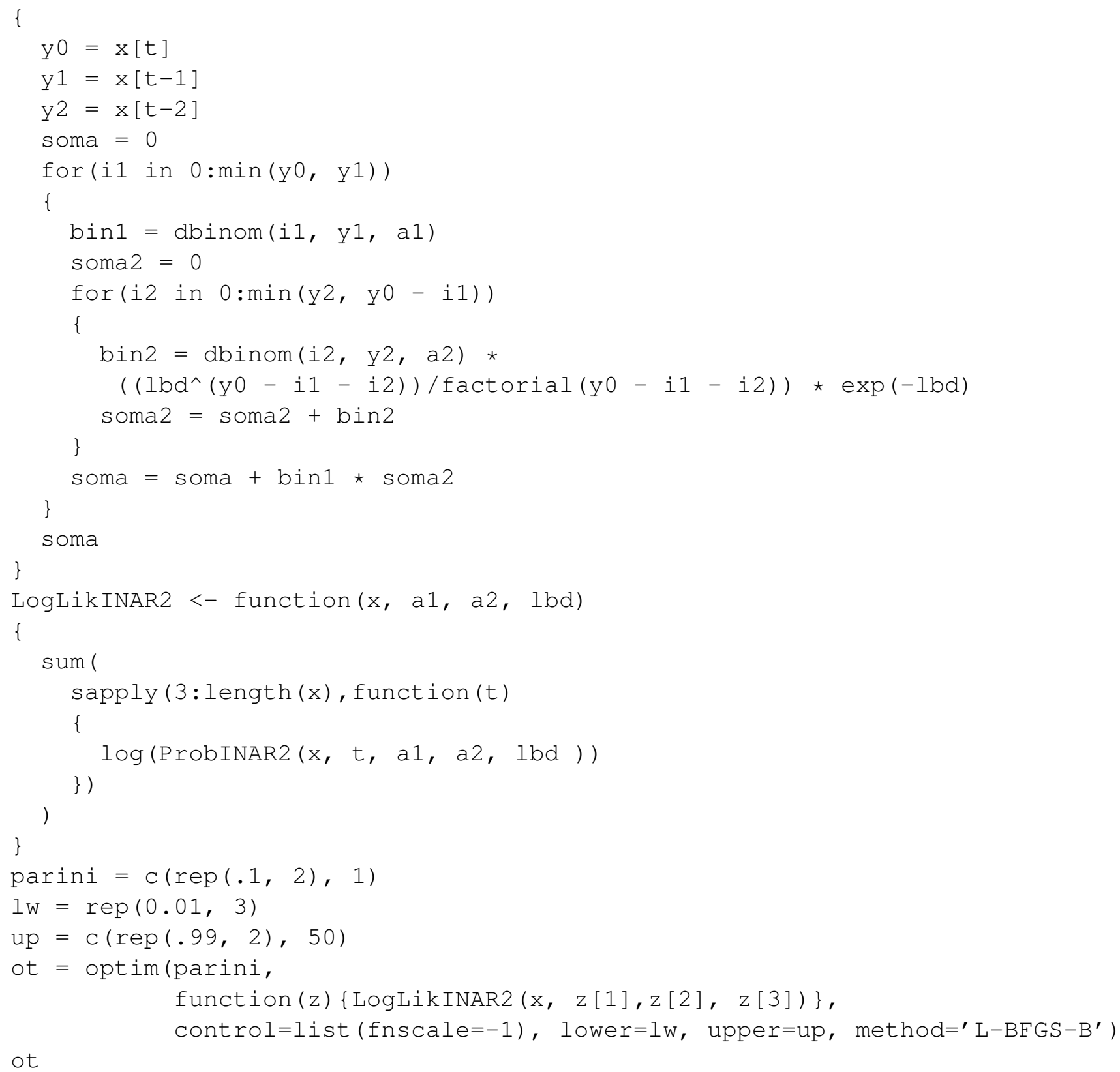


APÊNDICE A 


\section{Referências Bibliográficas}

Al-Osh e Alzaid (1987) M. A. Al-Osh e A. A. Alzaid. First-order integer-valued autoregressive (INAR(1)) process. Journal of time series analysis, 8:261-275. Citado na pág. 9, 10, 13

Alzaid e Al-Osh (1990) A.A Alzaid e M. Al-Osh. An integer-valued pth-order autoregressive structure (INAR(p)) process. Journal of Applied Probability, 27:314-324. Citado na pág. 9, 10

Box et al. (2008) George E. P. Box, Gwilym M. Jenkins e Gregory C. Reinsel. Time Series Analysis: Forecasting and Control. Wiley, fourth ed. Citado na pág. 9, 69

Garbade (1977) K. Garbade. Two methods for examinng the stability of regression coefficients. Journal of American Statistics Association, 72:54-63. Citado na pág. 39

Jacobs e Lewis (1983) P. A. Jacobs e P. A. W. Lewis. Stationary discrete autoregressive-moving average time series generated by mixtures. Journal of Time Series Analysis, 4:19-36. Citado na pág. 9

Jin-Guan e Li-Yuan (1991) Du Jin-Guan e Li-Yuan. The integer-valued autoregressive (INAR(p)) model. Journal of Time Series Analysis, 12:129-142. Citado na pág. 9, 10, 13, 68

Kendall (1953) M. G. Kendall. The analysis of economic time-series- part i: Prices. Journal of the Royal Statistic Society, páginas 11-25. Citado na pág. 39

Klimko e Nelson (1978) L. A. Klimko e P. L. Nelson. On conditional least square estimation for stochastic process. Annals of Statistics, 7:893-899. Citado na pág. 41

Morettin e Toloi (2006) Pedro A. Morettin e Clélia M. C. Toloi. Análise de Séries Temporais. Editora Edgard Blücher, segunda ed. Citado na pág. 5

Ruijun Bu e Hadri (2008) Brendan McCabe Ruijun Bu e Kaddour Hadri. Maximum likelihood estimation of higher-order integer-valued autoregressive processes. Journal of Time Series Analysis, 29:973-994. Citado na pág. 13, 17

Steutel e Harn (1979) F. W. Steutel e K. Van Harn. Discrete analogues of self-decomposability and stability. The Annals of Probability, 5:893-899. Citado na pág. 8

Zheng et al. (2006) H. Zheng, Ishwar V. Basawa e Somnath Datta. Inference for p-order random coefficient integer-valued autoregressive process. Journal of Time Series Analysis, 27:411-440. Citado na pág. 39, 40, 43

Zheng et al. (2007) H. Zheng, Ishwar V. Basawa e Somnath Datta. First-order random coefficient integer-valued autoregressive process. Journal of Statistical Planning and Inference, 173:212-229. Citado na pág. 39 